\title{
On evaluating stent-artery interaction in abdominal aortic stent grafting
}

An in-depth analysis of longitudinal and pulsatility related behavior

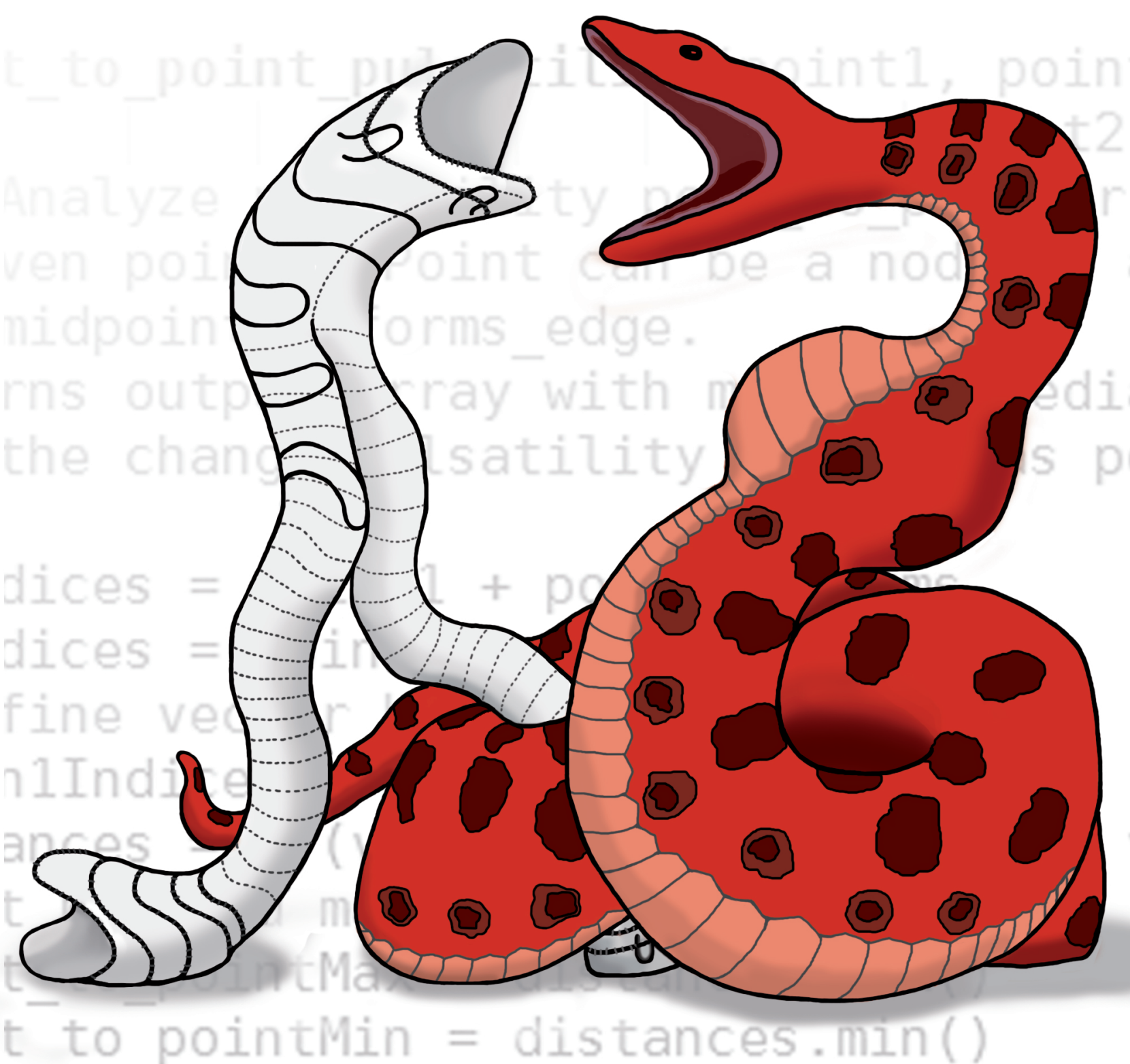

Maaike Koenrades 



\section{On evaluating stent-artery interaction in abdominal aortic stent grafting}

An in-depth analysis of longitudinal and pulsatility related behavior

Maaike Anne Koenrades 


\section{Members of the Graduation Committee}

\section{Chairman:}

Prof.dr. J.L. Herek (University of Twente)

\section{Promoters:}

Prof.dr. R.H. Geelkerken (University of Twente)

Prof.dr.ir. C.H. Slump (University of Twente)

\section{Co-promoter:}

Prof.dr.ir. B.J. Geurts (University of Twente)

\section{Members:}

Prof.dr.ir. S. Manohar (University of Twente)

Prof.dr. M.M.P.J. Reijnen (University of Twente)

Prof.dr. J.J. Fütterer (University of Twente)

Prof.dr. C.J.A.M. Zeebregts (University Medical Center Groningen)

Prof.dr. M.R.H.M. van Sambeek (Eindhoven University of Technology)

\section{-On evaluating stent-artery interaction in abdominal aortic stent grafting- An in-depth analysis of longitudinal and pulsatility related behavior}

Academic thesis, University of Twente, Enschede, the Netherlands, with a summary in Dutch.

$\begin{array}{ll}\text { Author: } & \text { Maaike A. Koenrades } \\ \text { Cover design: } & \text { Anne M. Leferink } \\ \text { Printed by: } & \text { IPSKAMP - www.ipskampprinting.nl } \\ \text { ISBN: } & 978-90-365-4875-5 \\ \text { DOI: } & 10.3990 / 1.9789036548755\end{array}$

Copyright (C) Maaike A. Koenrades, 2019

All rights reserved. No part of this publication may be reproduced or transmitted in any form or by any means without written permission of the author.

The author gratefully acknowledges financial support for the publication of this thesis by: Medisch Spectrum Twente; Stichting Wetenschappelijk Onderzoek Chirurgen Enschede (SWOCE); Multi-modality Medical Imaging (M3I) group, University of Twente; Robotics and Mechatronics (RaM) group, University of Twente; ChipSoft; Terumo Aortic.

Financial support by the Dutch Heart Foundation for the publication of this thesis is gratefully acknowledged.

The research described in this thesis was supported by research grants from Vascutek Terumo, trading as Terumo Aortic, Endologix Inc., and by a PPP Allowance made available by Health Holland, Top Sector Life Sciences \& Health, to stimulate public-private partnerships. 


\title{
ON EVALUATING STENT-ARTERY INTERACTION IN ABDOMINAL AORTIC STENT GRAFTING
}

AN IN-DEPTH ANALYSIS OF LONGITUDINAL AND PULSATILITY RELATED

BEHAVIOR

\section{DISSERTATION}

\author{
to obtain
}

the degree of doctor at the University of Twente, on the authority of the rector magnificus,

Prof.dr. T.T.M. Palstra,

on the account of decision of the graduation committee,

to be publicly defended

on Friday, November $1^{\text {st }} 2019$ at 14.45 hours

by

Maaike Anne Koenrades

born on May $9^{\text {th }}, 1989$

in Purmerend, the Netherlands 
This dissertation has been approved by:

Prof.dr. R.H. Geelkerken (Promoter)

Prof.dr.ir. C.H. Slump (Promoter)

Prof.dr.ir. B.J. Geurts (Co-promoter) 



\section{Table of Contents}

1 General introduction $\quad 1$

I Geometric changes over time $\quad 11$

2 Evolution of the proximal sealing rings of the Anaconda stent-graft after endovascular aneurysm repair

3 Geometric remodeling of the perirenal aortic neck at and adjacent to the double sealing ring of the Anaconda stent-graft after endovascular aneurysm repair

4 Peak and valley alignment of the Anaconda saddle-shaped sealing rings after endovascular aortic aneurysm repair: Implications for device positioning and sealing length

5 Geometric changes in Anaconda endograft limbs after endovascular aneurysm repair: A potential predictor for limb occlusion

II Cardiac cycle related behavior

6 Quantitative stent-graft motion in ECG-gated CT by image registration and segmentation: In vitro validation and preliminary clinical results

7 Electrocardiogram gated computed tomography quantification of sealing ring dynamics after endovascular aneurysm repair in patients treated with an Anaconda stent-graft

8 Dynamic computed tomography angiography analysis of cardiac pulsatility-induced motion and deformation after endovascular aneurysm sealing with chimney grafts

9 Future outlook: A mathematical approach to validate image registration for motion estimation 
10 General discussion 181

Summary (Dutch) 191

List of Publications 195

Biography 197

Dankwoord 199 



\section{Chapter 1}

\section{General introduction}

\section{Description of the condition}

In the aging society many elderly face cardiovascular diseases. Abdominal aortic aneurysm (AAA) is a condition in which a segment of the abdominal aorta, commonly in the infrarenal aorta[1], becomes permanently dilated (balloon-like bulge $>3 \mathrm{~cm}$ in diameter) by pathological weakening of the aortic wall[2,3]. AAA affects approximately $4.1 \%$ to $14.2 \%$ of men and $0.4 \%$ to $6.2 \%$ of women[4]. An AAA is considered infrarenal if a segment of normal (undilated) aorta exists between the AAA and the renal arteries. This segment is defined the aortic neck. In case the AAA originates just after or at the origin of the renal arteries it is considered juxtarenal or pararenal. The pathogenesis of an AAA is complex and multifactorial. It involves both genetic and environmental factors that lead to proteolytic degradation of aortic wall components. If left untreated, enlargement of the AAA under pulsatile pressure can result in rupture when the wall tension exceeds the tensile strength of the aortic wall, usually with fatal consequences (estimated $74 \%$ mortality rate[5]).

A

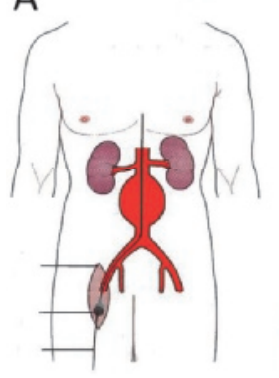

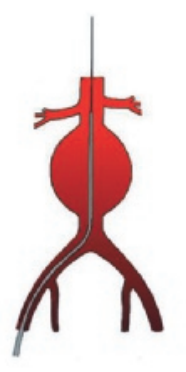
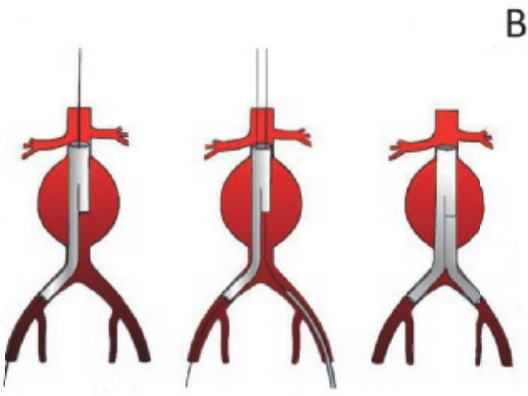

B

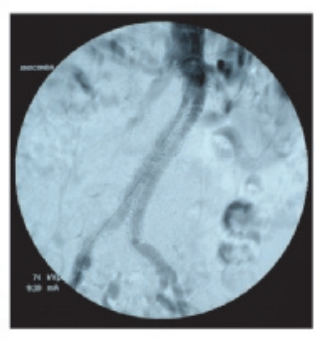

Figure 1. (A) Illustration of EVAR with modular stent-graft deployment (image courtesy of Droc et al[2]. (B) Intraoperative angiographic image of EVAR with an Anaconda stent-graft (Terumo Aortic, Inchinnan, Scotland, UK). 


\section{Current status of endovascular aneurysm repair}

Open surgical repair (OSR) has long been the treatment for AAA repair, starting in the 1950s. OSR is a highly invasive surgical procedure that requires cross clamping of the aorta for interposition of a hand-sewn aortic prosthesis. The 30-day mortality is high $(3.5-4.7 \%[6])$ but the long-term durability of OSR is excellent in patients with low surgical risk[7]. Even after introduction of endovascular aneurysm repair (EVAR) in 1988 by Volodos[8] and in 1991 by Parodi[9], it took a long time for EVAR to become widely accepted. EVAR is a less invasive procedure performed by endovascular insertion of collapsed stent-graft components (composed of fabric and metal stents) over a guidewire through a (femoral) access artery (Figure 1). After deployment, the stent-graft should exclude the aneurysm from blood flow by making a seal against the nondilated vessel wall in the proximal (aortic neck) and distal (iliac) landing zones. Nowadays, the less invasive nature makes EVAR the method of choice in elderly patients and patients with considerable comorbidities. Since 1999, reports of large randomized controlled trials have shown an evident perioperative survival benefit for EVAR compared to OSR[10,11]. However, long-term results of EVAR are shown to be similar to that of OSR, including quality of life and costeffectiveness[10,11]. This is related to the relatively high rate of reintervention to solve problems such as migration (caudal device displacement), endoleak (blood flow into the aneurysm), occlusion (kinking or graft thrombosis), and structural device failure (metal fracture, fabric tear)[6,12-14]. These failure modes result in the need for lifelong surveillance after EVAR[15]. Fortunately, much has changed since the large clinical trials. Dedicated efforts by device manufacturers and physicians have led to the introduction of second and third generation devices to eliminate sources of failure. Current available devices fail less often than their predecessors but still come with a reintervention rate of $10-20 \%$ over time[16]. In addition, any possible long-term benefit remains unproven[13].

\section{Why does durability remain an issue?}

While initially approximately $40 \%$ of patients were excluded for EVAR due to aortic neck anatomy constraints or unsuitable access vessels[17], nowadays approximately $80 \%$ of patients is treated with EVAR[18]. Overall, the application of EVAR is expanding to patients with increasingly complex and anatomically challenging anatomy, even outside the instructions for use (IFU), but also to younger, low-risk patients with a long life expectancy, which further challenges the long-term outcome. Moreover, by introducing new or modified devices to overcome current failure modes or to avail a larger number of patients we may expect new complications and challenges to be dealt with[10]. Additionally, structural device failure may take longer to manifest as material improvements have been incorporated to increase resilience for the continuous cyclic loading of the metal stents during the cardiac cycle[19]. In the long-term, device fatigue remains one of the most concerning failure modes since it may encompass hook or stent fractures and fabric tears leading to dislocation and endoleak[14]. Furthermore, aortic neck dilatation may lead to a loss of contact between the aortic wall and the stent-graft with subsequent type Ia endoleak, migration and the need for reintervention[20]. Such dilatation may be due to the radial force of self-expanding 
stent-grafts but also due to ongoing disease progression, which could occur any time after EVAR.

In summary, reservations about the long-term durability of EVAR persist as its failure modes remain relevant.

\section{What is needed to improve treatment durability?}

The outcome of EVAR largely depends on how a device is used (e.g., sizing, positioning, patient selection) and is able to resist or adapt to the dynamic endovascular environment[21]. Each stent-graft has the same ultimate goal, i.e., to exclude the aneurysm from blood flow and prevent rupture. Yet, stent-graft designs can strongly differ with respect to, for example, the material, the stent frame structure, the fixation zone and the sealing mechanism. So how should a physician determine which device to use and how to use it to prevent failure? In turn, device optimization by manufacturers inquires knowledge about morphological changes and pulsatility-induced changes of the implanted devices for experimental evaluations (e.g., fatigue test banks) and computer simulations (e.g., finite element analysis) to assess device durability.

EVAR surveillance currently aims at detecting failure modes (e.g., endoleak, migration, aneurysm growth, occlusion) by static computed tomography (CT) angiography imaging or duplex ultrasound. However, the processes underlying failure are often not well understood and therefore difficult to recognize during follow-up. Gaining insight in causes of failure and early signs of failure can help to identify patients at risk, which allows for tailored follow-up schemes and early intervention with relatively simple endovascular procedures. Such processes can be highly device specific and require an in-depth understanding of the natural 'behavior' that occurs in vivo, both over time and during the cardiac cycle. Hence, knowledge of the behavior of any device in use is essential to allow for liable durability tests and to design newer generation devices that well match the patient's endovascular environment. This calls the research community to investigate the ongoing stent-artery interaction in vivo and consequently for the development of methods to achieve this. 


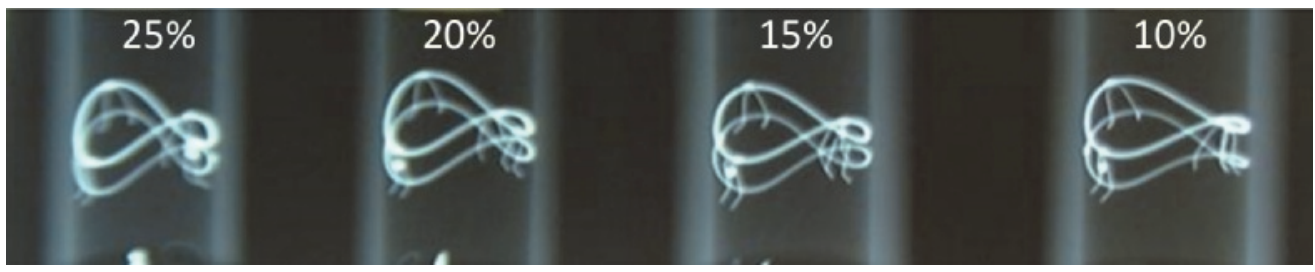

Figure 2. Shape of the Anaconda stent-graft double sealing ring at various degrees of oversizing (image courtesy of Terumo Aortic).

\section{Outline of the thesis}

The objective of this thesis is to develop, validate and apply methods to broaden our understanding of in-vivo stent-graft behavior in terms of longitudinal changes and changes that occur during the cardiac cycle. This work aims to provide insight to support physicians in their decision making and device manufacturers to validate, refine or redesign stent-grafts that can endure the endovascular environment. Both commercially available software and advanced in-house developed image processing algorithms were used. The methods were primarily applied to a cohort of patients that underwent EVAR with an Anaconda AAA stent-graft (Terumo Aortic, Inchinnan, Scotland, UK). Electrocardiogram-gated CT scans were prospectively collected by a meticulous study protocol during a 2-year follow-up period (Longitudinal Study to Pulsatility and Expansion in Aortic Stent-grafts; Trialregister.nl; identifier NTR4276). In the first part of this thesis longitudinal changes in the geometry of the device and vessel are described. The second part addresses cardiac-pulsatility induced behavior, including validation of methods and assessment of pulsatile changes in various device configurations that occur during the cardiac cycle.

\section{Part I: Geometric changes over time}

The Anaconda stent-graft is one of the current commonly used infrarenal AAA stentgrafts[16,22]. It was first introduced to the market in 2005 and has since been shown to perform well in clinical use[23-31]. Still, similar to any stent-graft, concerns about the durability remain and reinterventions do still occur due to complications such as type Ia endoleak[32] and limb occlusion[31]. Moreover, knowledge about the device behavior after implantation is limited as most studies have investigated clinical outcome.

The Anaconda stent-graft has a double nitinol stent-ring for proximal sealing and fixation. When implanted in the aortic neck, the rings assume the shape of a 'saddle' with peaks and valleys (Figure 1B). The shape of the saddle is directly related to the degree of oversizing (Figure 2). The long-term integrity of the seal depends principally on the sizing, the conformability of the rings, and the resilience of the aortic wall to the radial force of the stent-rings. Over time, the stent-rings are known to expand whereby the saddle shape flattens. However, the evolution of this change was not fully understood. Additionally, dilation of the aortic neck can jeopardize the seal and positional stability by which the expansion of these sealing rings may raise concerns 
for the long-term outcome. In chapter 2, the expansion and conformability of the stent-rings was investigated while in chapter 3 the geometric remodeling of the aortic neck was addressed. In the latter study, changes in aortic size were evaluated above, at and below the sealing rings to understand whether dilatation was localized to the level of the sealing rings or whether the complete neck may have been affected.

Besides expansion, the change in saddle shape can influence the position of the stent-rings within the aortic neck. With this change in geometry, the valleys may migrate upstream and the peaks downstream. Depending on how the sealing rings were positioned, this could lead to a loss of sealing length or inadvertent coverage of the renal artery ostia. Both may have serious consequences including type Ia endoleak or renal failure. The saddle-shape allows the peaks to be placed pararenally with the valleys positioned below the renal arteries, which could allow higher placement in the aortic neck that may benefit both sealing length and positional stability. However, in order to safely effectuate such device deployment (currently outside the IFU), the extent of peak and valley displacement must be understood in detail. In chapter 4, these changes have been investigated throughout the course of saddle alignment.

Limb occlusion after EVAR is a common reason for reintervention[31]. The modular limbs of the Anaconda stent-graft comprise independent O-shaped stent-rings sewn onto a woven polyester graft fabric. This design increases the conformability and compatibility for tortuous iliac anatomy. Yet, it also allows for inwards folding of the graft fabric, which may contribute to the emergence of limb embolization or thrombosis by disturbing flow patterns. Changes in the patient's anatomy several months to years after EVAR may induce changes in the initial limb geometry (e.g., length, angulation) that could result in such inwards folding of graft fabric. Consequently, it is of interest to understand these changes to identify potential predictors of limb occlusion and to further improve the stent-graft design. Chapter 5 was established to assess geometric changes in the limbs of the Anaconda stent-graft.

\section{Part II: Cardiac cycle related behavior}

Measurement of stent-graft motion in the abdominal aorta requires a method that can appreciate subtle but complex 3-dimensional motions. For this purpose, a methodology that combines image registration and segmentation techniques has previously been proposed[33,34]. In this thesis, we have implemented, evaluated and expanded the algorithms to study pulsatility-induced behavior of stent-grafts.

In chapter 6 , the accuracy and sensitivity of this method is evaluated by performing phantom experiments with a motion generation device. Furthermore, the method was tested in vivo in various clinical cases for infrarenal and juxtarenal AAA including an infrarenal Anaconda device, a fenestrated Anaconda device with visceral grafts, an Endurant device with suprarenal fixating stents (Medtronic, Santa Rosa, CA, USA) and a Nellix sac sealing endosystem (Endologix, Irvine, CA, USA) combined with chimney grafts.

As stent-grafts are implanted in a dynamic pulsatile environment, the fixation and seal as well as the mechanical stability of the device are continuously challenged. The Anaconda device was designed to counteract the pulsatility of the aortic vessel by the radial force of the proximal stent-rings. However, pulsatile distension and deformation 
of the stent-rings had not yet been investigated in a clinical setting. Such knowledge is important for fatigue life evaluation and design verification. In addition, it may be important for sizing and failure prediction during follow-up. Therefore, in chapter 7 we evaluate the cyclic adaptations of the Anaconda sealing rings during the cardiac cycle.

The methods were further expanded and applied in chapter 8 to evaluate the Nellix chimney device configuration more extensively. EndoVascular aneurysm sealing (EVAS) was introduced in 2011 as an alternative for conventional EVAR to reduce reintervention rates[35]. Different from self-expanding stent-grafts that use radial force and hooks/barbs, the Nellix endosystem uses polymer filled endobags to fixate two balloon-expandable stents that seal the entire aneurysm. Combined with chimney grafts, this technique can be used to treat para- and juxtarenal AAA. The chimney grafts that run parallel to the stents and cannulate the side branches are also stabilized by the polymer. However, cardiac-pulsatility induced motions may jeopardize the sealing and fixation if the individual stents and chimney grafts do not move as a single unit. In addition, the stent components must be able to withstand the repetitive stresses posed by the pulsatile blood flow to maintain mechanical stability. Even though the EVAS procedure was first considered simple and quick with wide patient applicability, the inclusion criteria had to be altered multiple times to reduce the presentation of failure modes[35,36]. Moreover, since long-term data on clinical outcome is not yet available, it is of utmost importance for engineers to understand the motions that occur in vivo to allow for adequate durability tests and design improvements. Consequently, the proximal stability of this configuration during the cardiac cycle was investigated in chapter 8 .

The development of quantitative methods is accompanied by its evaluation and validation for a particular application to ensure reliability of measurements. Validation of registration algorithms is a challenging problem due to a lack of ground truth in clinical image data[37]. Phantom experiments are valuable to evaluate registration performance as it allows for evaluation of a target registration error. However, the subtle deformations that may occur in the abdominal aorta are difficult to mimic in a physical phantom. Synthetically deformed data provides another means to evaluate registration accuracy. In chapter 9, we discuss a mathematical approach for the assessment of registration accuracy by transforming real application-specific patient data. 


\section{References}

1. Jongkind V, Yeung KK, Akkersdijk GJM, Heidsieck D, Reitsma JB, Tangelder GJ, et al. Juxtarenal aortic aneurysm repair. J Vasc Surg. 2010;52:760-7.

2. Droc I, Raithel D, Calinescu B. Abdominal Aortic Aneurysms - Actual Therapeutic Strategies. In: Murai Y, editor. Aneurysm. [Rijeka]: IntechOpen; 2012.

3. Sakalihasan N, Limet R, Defawe OD. Abdominal aortic aneurysm. Lancet. 2005;365:1577-89.

4. Cornuz J, Sidoti Pinto C, Tevaearai H, Egger M. Risk factors for asymptomatic abdominal aortic aneurysm. Eur J Public Health. 2004;14:343-9.

5. Reimerink JJ, Piros L, Koelemay MJW, Balm R, Legemate DA. Systematic review and metaanalysis of population-based mortality from ruptured abdominal aortic aneurysm. Br J Surg. 2013;100 11:1405-13.

6. Drury D, Michaels JA, Jones L, Ayiku L. Systematic review of recent evidence for the safety and efficacy of elective endovascular repair in the management of infrarenal abdominal aortic aneurysm. Br J Surg. 2005;92:937-46.

7. Rutherford R. Open versus endovascular stent graft repair for abdominal aortic aneurysms: an historical view. Semin Vasc Surg. 2012;25:39-48.

8. Volodos' NL, Shekhanin VE, Karpovich IP, Troian VI, Gur'ev IA. [A self-fixing synthetic blood vessel endoprosthesis]. Vestn Khir Im I I Grek. 1986;137:123-5.

9. Parodi JC, Palmaz JC, Barone HD. Transfemoral Intraluminal Graft Implantation for Abdominal Aortic Aneurysms. Ann Vasc Surg. 1991;5:491-9.

10. Propper BW, Abularrage CJ. Long-term safety and efficacy of endovascular abdominal aortic aneurysm repair. Vasc Health Risk Manag. 2013;9:135-41.

11. Powell JT, Sweeting MJ, Ulug P, Blankensteijn JD, Lederle FA, Becquemin J-P, et al. Meta-analysis of individual-patient data from EVAR-1, DREAM, OVER and ACE trials comparing outcomes of endovascular or open repair for abdominal aortic aneurysm over 5 years. BJS. 2017;104:166-78.

12. Franks SC, Sutton AJ, Bown MJ, Sayers RD. Systematic Review and Meta-analysis of 12 Years of Endovascular Abdominal Aortic Aneurysm Repair. Eur J Vasc Endovasc Surg. 2007;33:154-71.

13. Bahia SS, Holt PJE, Jackson D, Patterson BO, Hinchliffe RJ, Thompson MM, et al. Systematic Review and Meta-analysis of Long-term survival after Elective Infrarenal Abdominal Aortic Aneurysm Repair 1969-2011: 5 Year Survival Remains Poor Despite Advances in Medical Care and Treatment Strategies. Eur J Vasc Endovasc Surg. 2015;50:320-30.

14. Jacobs TS, Won J, Gravereaux EC, Faries PL, Morrissey N, Teodorescu VJ, et al. Mechanical failure of prosthetic human implants: A 10-year experience with aortic stent graft devices. J Vasc Surg. 2003;37:16-26.

15. Chaikof EL, Dalman RL, Eskandari MK, Jackson BM, Lee WA, Mansour MA, et al. The Society for Vascular Surgery practice guidelines on the care of patients with an abdominal aortic aneurysm. J Vasc Surg. 2018;67:2-77.e2.

16. Jonker LT, de Niet A, Reijnen MMPJ, Tielliu IFJ, Zeebregts CJ. Mid- and Long-Term Outcome of Currently Available Endografts for the Treatment of Infrarenal Abdominal Aortic Aneurysm. Surg Technol Int. 2018;33:239-50. 
17. Harris PL, Vallabhaneni SR, Desgranges P, Becquemin JP, Van Marrewijk C, Laheij RJF. Incidence and risk factors of late rupture, conversion, and death after endovascular repair of infrarenal aortic aneurysms: The EUROSTAR experience. J Vasc Surg. 2000;32:739-49.

18. Schermerhorn ML, Bensley RP, Giles KA. Changes in Abdominal Aortic Aneurysms Rupture and Short-Term Mortality, 1995-2008: A Retrospective Observational Study. J Vasc Surg. 2012;56:1809-10.

19. Corbett TJ, Callanan A, Morris LG, Doyle BJ, Grace PA, Kavanagh EG, et al. A Review of the in Vivo and in Vitro Biomechanical Behavior and Performance of Postoperative Abdominal Aortic Aneurysms and Implanted Stent-Grafts. J Endovasc Ther. 2008;15:468-84.

20. Kouvelos GN, Oikonomou K, Antoniou GA, Verhoeven ELG, Katsargyris A. A systematic review of proximal neck dilatation after endovascular repair for abdominal aortic aneurysm. J Endovasc Ther. 2017;24:59-67.

21. Beebe HG. Lessons learned from aortic aneurysm stent graft failure; observations from several perspectives. Semin Vasc Surg. 2003;16:129-38.

22. Belvroy VM, Houben IB, Trimarchi S, Patel HJ, Moll FL, Van Herwaarden JA. Identifying and addressing the limitations of EVAR technology. Expert Rev Med Devices. 2018;15:541-54.

23. Freyrie A, Gargiulo M, Testi G, Faglioli G, Rossi C, Mauro R, et al. Midterm results of Anaconda ${ }^{\mathrm{TM}}$ infrarenal aortic endografts: a single-center prospective study. Ital J Vasc Endovasc Surg. $2009 ; 16: 1-7$.

24. Freyrie A, Testi G, Faggioli GL, Gargiulo M, Giovanetti F, Serra C, et al. Ring-stents supported infrarenal aortic endograft fits well in abdominal aortic aneurysms with tortuous anatomy. J Cardiovasc Surg (Torino). 2010;51:467-74.

25. Freyrie A, Gallitto E, Gargiulo M, Faggioli G, Massoni CB, Mascoli C, et al. Results of the endovascular abdominal aortic aneurysm repair using the Anaconda aortic endograft. J Vasc Surg. 2014;60:1132-9.

26. Saratzis N, Melas N, Saratzis A, Lazarides J, Ktenidis K, Tsakiliotis S, et al. Anaconda aortic stent-graft: single-center experience of a new commercially available device for abdominal aortic aneurysms. J Endovasc Ther. 2008;15:33-41.

27. Karkos CD, Kapetanios DM, Anastasiadis PT, Grigoropoulou FS, Kalogirou TE, Giagtzidis IT, et al. Endovascular Repair of Abdominal Aortic Aneurysms with the Anaconda TM Stent Graft: Mid-term Results from a Single Center. Cardiovasc Intervent Radiol. 2015;38:1416-24.

28. Majumder B, Urquhart G, Edwards R, Irshad K, Velu R, Reid DB. Early clinical experience with the Anaconda re-deployable endograft in 106 patients with abdominal aortic aneurism: the west of Scotland Anaconda registry. Scott Med J. 2012;57:61-4.

29. Rödel SGJ, Zeebregts CJ, Huisman AB, Geelkerken RH. Results of the Anaconda endovascular graft in abdominal aortic aneurysm with a severe angulated infrarenal neck. J Vasc Surg. 2014;59:1495-1501.e1.

30. Rödel SGJ, Geelkerken RH, Prescott RJ, Florek HJ, Kasprzak P, Brunkwall J. The Anaconda AAA stent graft system: 2-year clinical and technical results of a multicentre clinical evaluation. Eur J Vasc Endovasc Surg. 2009;38:732-40.

31. Rödel SGJ, Zeebregts CJ, Meerwaldt R, van der Palen J, Geelkerken RH. Incidence and treatment of limb occlusion of the Anaconda endograft after endovascular aneurysm repair. J Endovasc 
Ther. 2018;26:113-20.

32. Vukovic E, Czerny M, Beyersdorf F, Wolkewitz M, Berezowski M, Siepe M, et al. Abdominal aortic aneurysm neck remodeling after Anaconda stent graft implantation. J Vasc Surg. 2018;68:13541359.e2.

33. Klein A. A tool for studying the motion of stent grafts in AAA. Segmentation and motion estimation of stent grafts in abdominal aortic aneurysms. [Enschede]: PhD Dissertation, University of Twente; 2011. p. 121-37.

34. Klein A, Renema W, Vliet JA, Oostveen LJ, Hoogeveen Y, Schultze Kool LJ, et al. Motion Calculations on Stent Grafts in AAA. In: Grundmann RT, editor. Diagnosis, Screening and Treatment of Abdominal, Thoracoabdominal and Thoracic Aortic Aneurysms. [Rijeka]: InTechOpen; 2011. p. $125-44$.

35. Reijnen MMPJ, Holden A. Status of Endovascular Aneurysm Sealing After 5 Years of Commercial Use. J Endovasc Ther. 2018;25:201-6.

36. Carpenter JP, Lane JS, Trani J, Hussain S, Healey C, Buckley CJ, et al. Refinement of anatomic indications for the Nellix System for endovascular aneurysm sealing based on 2-year outcomes from the EVAS FORWARD IDE trial. J Vasc Surg. 2018;68:720-730.e1.

37. Liu Z, Deng X, Wang G. Accuracy Validation for Medical Image Registration Algorithms: a Review. Chinese Med Sci J. 2012;27:176-81. 



\section{Part I}

Geometric changes over time 




\section{Chapter 2}

\section{Evolution of the proximal sealing rings of the Anaconda stent-graft after endovascular aneurysm repair}

Maaike A. Koenrades ${ }^{a, b}$, Almar Klein ${ }^{b}$, Anne M. Leferink ${ }^{b}$, Cornelis H. Slump ${ }^{b}$, and Robert H. Geelkerken $^{a, b}$

${ }^{a}$ Department of Vascular Surgery, Medisch Spectrum Twente, Enschede, the Netherlands

${ }^{b}$ MIRA Institute for Biomedical Engineering and Technical Medicine, University of Twente, Enschede, the Netherlands 


\section{Abstract}

Purpose: To provide insight into the evolution of the saddle-shaped proximal sealing rings of the Anaconda stent-graft after endovascular aneurysm repair (EVAR).

Methods: Eighteen abdominal aortic aneurysm patients were consecutively enrolled in a single-center, prospective, observational cohort study (LSPEAS; Trialregister.nl identifier NTR4276). The patients were treated electively using an Anaconda stentgraft with a mean $31 \%$ oversizing (range 17-47). According to protocol, participants were to be followed for 2 years, during which 5 noncontrast electrocardiogram-gated computed tomography scans would be conducted. Three patients were eliminated within 30 days (1 withdrew, 1 died, and a third was converted before stent-graft deployment), leaving 15 patients (mean age $72.8 \pm 3.7$ years; 14 men) for this analysis. Evolution in size and shape (symmetry) of both proximal infrarenal sealing rings were assessed from discharge to 24 months using dedicated postprocessing algorithms.

Results: At 24 months, the mean diameters of the first and second ring stents had increased significantly (first ring: $2.2 \pm 1.0 \mathrm{~mm}, \mathrm{p}<0.001$; second ring: $2.7 \pm 1.1$ $\mathrm{mm}, \mathrm{p}<0.001)$. At 6 months, the first and second rings had expanded to a mean $96.6 \% \pm 2.1 \%$ and $94.8 \% \pm 2.7 \%$, respectively, of their nominal diameter, after which the rings expanded slowly; ring diameters stabilized to near nominal size (first ring, $98.3 \% \pm 1.1 \%$; second ring, $97.2 \% \pm 1.4 \%$ ) at 24 months irrespective of initial oversizing. No type I or III endoleaks or aneurysm-, device-, or procedure- related adverse events were noted in follow-up. The difference in the diametric distances between the peaks and valleys of the saddle-shaped rings was marked at discharge but became smaller after 24 months for both rings (first ring: median 2.0 vs $1.2 \mathrm{~mm}$, $\mathrm{p}=0.191$; second ring: median 2.8 vs $0.8 \mathrm{~mm}$; $\mathrm{p}=0.013)$.

Conclusion: Irrespective of initial oversizing, the Anaconda proximal sealing rings radially expanded to near nominal size within 6 months after EVAR. Initial oval-shaped rings conformed symmetrically and became nearly circular through 24 months. These findings should be taken into account in planning and follow-up.

Keywords: Abdominal aortic aneurysm, endograft deployment, endovascular aneurysm repair, expansion, fixation, nitinol ring stent, proximal sealing, ring symmetry, stent-graft 


\section{Introduction}

In the past 20 years, the midterm results of endovascular aneurysm repair (EVAR) of abdominal aortic aneurysms (AAAs) have improved, resulting in broader application of this treatment and the commercialization of a multitude of stent-graft designs[1]. The long-term outcome of EVAR, though, still remains a concern[2] especially since treatment indications are expanding to include not only unfavorable AAA anatomies[3] but also younger, low-risk patients with a long life expectancy[4].

Durable proximal attachment and sealing are crucial for long-term integrity and depend on the interaction between the proximal stent-graft and the nonaneurysmal infrarenal or suprarenal aortic wall. A loss of contact with the wall can lead to endoleak and migration, which are the main reasons for reinterventions[5]. Self-expanding stent-grafts rely on a sufficient degree of oversizing to exert a continued outward pressure on the aortic wall in order to provide an adequate seal. Self-expanding stentgrafts may conform to the vessel should the aortic neck dilate, which is in contrast to balloon-expandable and sac anchoring devices that do not have such spring-like behavior.

Additionally, there are considerable differences in the sealing and fixation mechanisms among self-expanding stent-graft designs[1], including but not limited to suprarenal or infrarenal fixation, radial strength, structure of the wire frame, and number of hooks and barbs. Understanding the specific characteristics of each device is paramount in selecting the most appropriate device, size, and deployment technique for each individual patient anatomy and for appreciating potentially harmful adaptations of the aortic neck over time. In addition, such insight can encourage device manufacturers to improve devices to maximize durability.

The Anaconda AAA stent-graft (Vascutek, a Terumo company, Inchinnan, Scotland) is a self-expanding infrarenally fixating device with a proximal dual ring design that assumes a saddle shape with peaks and valleys when oversized and deployed in the aortic neck (Figure 1). The initial design of Lauterjung in 1996 has evolved to a device with favorable midterm results $[6-8]$, even in severely angulated anatomy $[9,10]$. From clinical observations, it appears that over time the rings expand and the saddle shape flattens $[11,12]$. However, the evolution of this change, the relation to oversizing, and the extent and symmetry of ring expansion have not been studied in detail. It is not known whether the rings expand uniformly in the directions of the peaks and valleys or whether the shape of the rings changes over time, which may be important for long-term integrity of the wall and seal.

The objective of the present study was to investigate the evolution of the postdeployment saddle shape of the Anaconda AAA stent-graft by prospectively evaluating changes in size and shape of the proximal sealing and fixation rings after EVAR.

\section{Methods}

Study design and patient sample From April 2014 to May 2015, asymptomatic patients $>70$ years old with an infrarenal AAA anatomically suitable for elective EVAR using an Anaconda AAA stent-graft were prospectively enrolled in a singlecenter, observational cohort study [Longitudinal study of pulsatility and expansion 


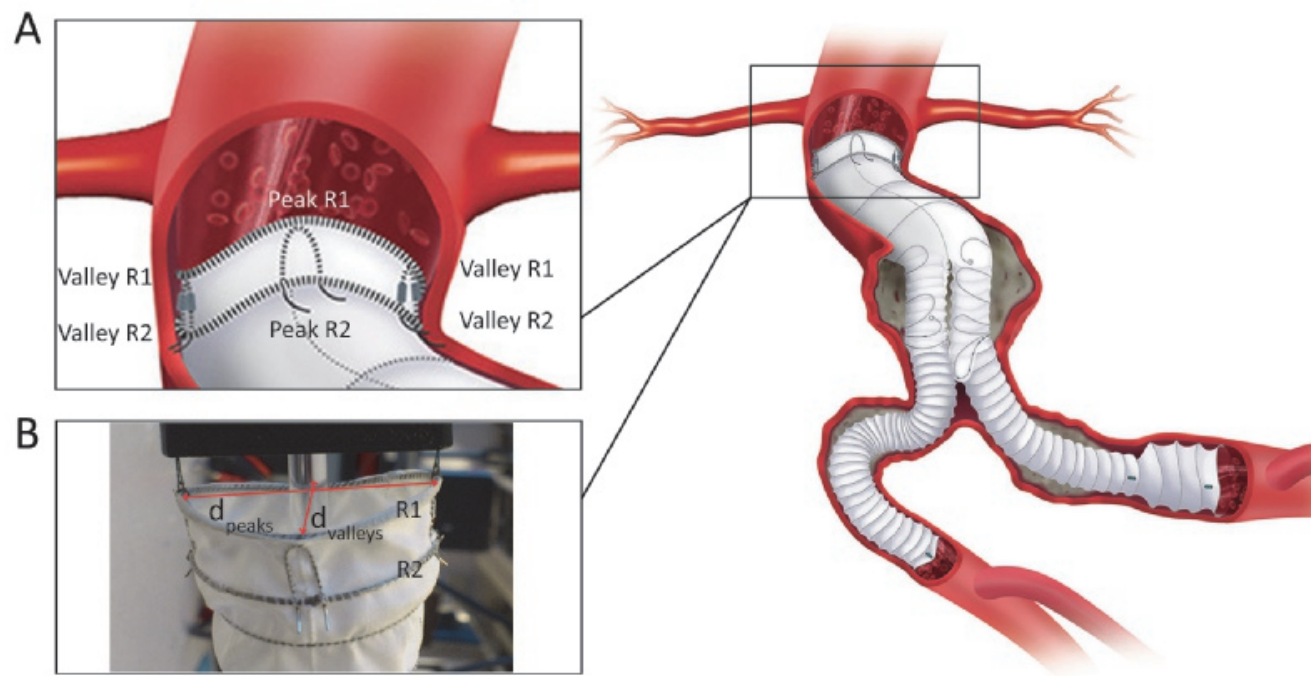

Figure 1. A representation (right image) of a deployed Anaconda stent-graft system for the treatment of infrarenal abdominal aortic aneurysm. (A) The proximal part of the main body showing the dual ring in a saddle configuration forming peaks and valleys. (B) Diametric distances analyzed in this study are labeled in the photograph for the first ring stent. R1, first ring stent; $\mathrm{R} 2$, second ring stent; $\mathrm{d}_{\text {peaks }}$, distance between peaks; $\mathrm{d}_{\text {valleys }}$, distance between valleys. (Schematic illustration was adapted with permission from Vascutek Ltd.)

in aortic stent-grafts (LSPEAS); registered on Trialregister.nl identifier NTR4276] designed to investigate factors influencing the success or failure of proximal stent-graft fixation and sealing. The study protocol was approved by the institutional review board. Written informed consent was obtained for each subject before participation in the study.

Patients were screened to evaluate their suitability for elective EVAR and inclusion in the trial. The screening consisted of a general health analysis, including the Society of Vascular Surgery[13] risk scores, as well as the American Society of Anesthesiologists classification[14]. Spiral computed tomography angiography (CTA) was performed according to standard practice to define aneurysm anatomy according to the EUROSTAR criteria[15,16]. By protocol, non-contrast electrocardiogram (ECG)gated CT scans were performed before intervention, before discharge, and after 1, 6, 12 , and 24 months of follow-up. In addition, after 1 month, participants underwent duplex ultrasound at the subsequent visits to evaluate the presence of endoleaks. Only patients who were able to comply with these requirements were eligible for the study.

Of the 18 patients enrolled in the LSPEAS trial during the observation period, 1 patient withdrew within 30 days, 1 patient died within 30 days (pulmonary embolism), and a third patient was converted to open repair owing to iliac access issues, leaving 15 patients (mean age $72.8 \pm 3.7$ years; 14 men) who completed the minimum 12-month follow-up (Figure 2). Patient characteristics and aneurysm characteristics of the 15 patients are summarized in Table 1. 


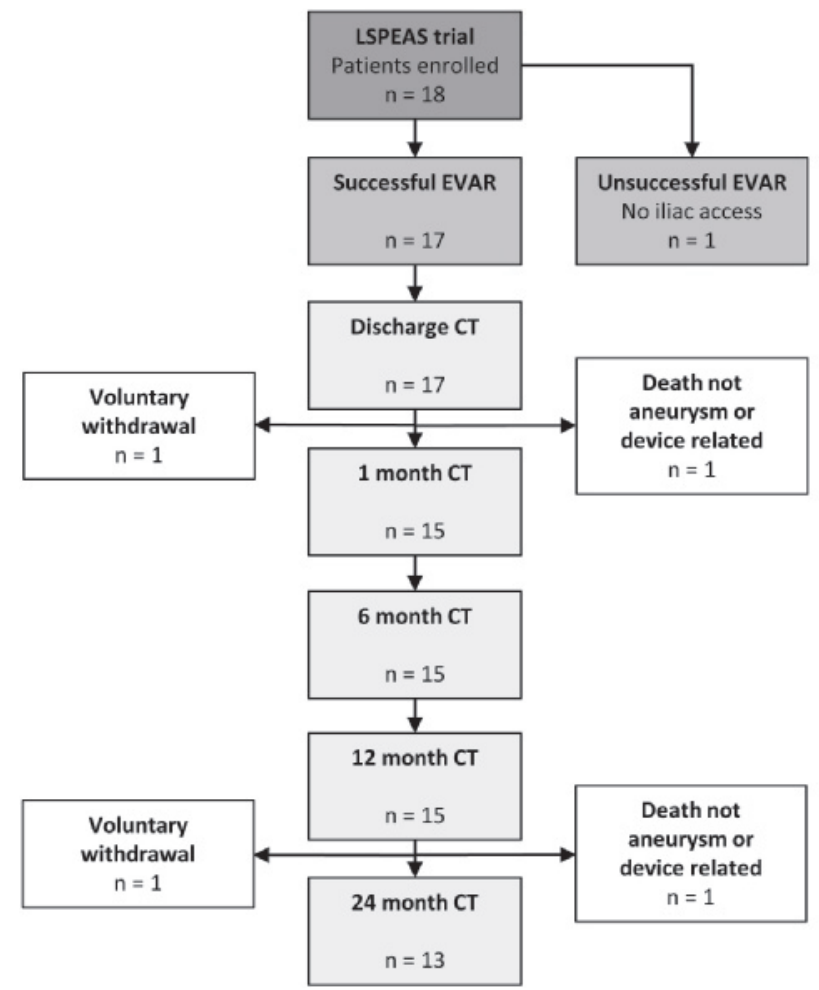

Figure 2. Chart showing the flow of patients enrolled in the LSPEAS (Longitudinal study of pulsatility and expansion in aortic stent-grafts) trial. CT, computed tomography; EVAR, endovascular aneurysm repair.

Patient demographic data and information on implanted stent-graft diameters were obtained from the patient registry. Stent-grafts were sized from inner wall diameters. Oversizing (diameter perpendicular to the flow axis) was increased in case of unfavorable neck anatomy, including reversed conical and short necks, and for inclined placement (i.e., nonperpendicular to the flow axis) in angulated necks, resulting in a broad range of initial oversizing (mean 31\%, range 17-47).

Device description The Anaconda AAA stent-graft system and implantation procedure have been extensively described elsewhere[6,8,15]. In short, the Anaconda stent-graft is a repositionable 3-piece endovascular graft for infrarenal fixation and consists of a woven polyester graft supported by independent nitinol ring stents that each comprise a single strand of wound nitinol wire, that is, a wire bundle (Figure 1). The proximal part of the main body consists of a self-expanding dual ring, which assumes the shape of a 'saddle' when oversized and constrained against the aortic wall. When unconstrained, the shape of the rings is circular. The first ring stent (R1) has a larger wire bundle diameter (higher number of wire turns) compared to the second ring stent (R2), resulting in a higher radial strength compared to R2. The 
Table 1. Patient and aneurysm characteristics. ${ }^{a}$

Demographics/risk factors

Age, y

$72.8(70-80)$

14

Body mass index, $\mathrm{kg} / \mathrm{m}^{2}$

ASA grade I / II / III

$26.8(22.2-34.7)$

Smoking

$2 / 12 / 1$

Hypertension

7

14

Hyperlipidemia

12

Cardiac disease

7

Stroke / TIA

$1 / 2$

Renal disease

1

Pulmonary disease

2

Aneurysm

Eurostar $^{b}$ A / B / C / D / E

Infrarenal neck diameters, $\mathrm{mm}$

$1 / 8 / 1 / 4 / 1$

$22(18-28)$

D2a

$22(18-28)$

$\mathrm{D} 2 \mathrm{~b}$

$23(19-29)$

D2c

$23(19-29)$

Neck shape ${ }^{c}$ I / II / III / IV

$9 / 4 / 1 / 1$

Neck length, mm

$35(20-75)$

Circumferential calcification

D2a / b / c, \%

D2a / b / c > $25 \%$

$50 / 60 / 80$
2

Luminal thrombus

D2a / b / c, \%

D2a / b / c > $25 \%$

$0 / 30 / 35$

1

Infrarenal neck angulation, deg

$>60 \mathrm{deg}$

Maximum AAA diameter

Main device diameters, $\mathrm{mm}$

25.5 (OLB 25)

28 (OLB 28)

30.5 (OLB 30)

32 (OLB 32)

34 (OLB 34)

$43(0-110)^{d}$

4

$60(40-70)^{e}$

Oversizing, ${ }^{f} \%$

1

5

6

1

2

$31(17-47)$

Abbreviations: AAA, abdominal aortic aneurysm; ASA, American Society of Anesthesiologists; OLB, main body device size; TIA, transient ischemic attack.

${ }^{a}$ Continuous data are presented as the means (range); categorical data are given as the counts.

${ }^{b}$ EUROSTAR AAA morphology[16].

${ }^{c}$ Neck shape according to Balm et al.[17]

${ }^{d}$ Two patients with angulation $>90^{\circ}$ were positioned with $90^{\circ}$ rotation (saddle peaks in lateral direction and valleys and legs in anteroposterior direction).

${ }^{e}$ One AAA $<50 \mathrm{~mm}$ but with 38 -mm iliac aneurysms (EUROSTAR category D)

${ }^{f}$ Device size was based on inner wall diameters. 
larger the main body size, the larger the wire bundle diameter (R1, 0.7-1.0 mm; R2, $0.5-0.7 \mathrm{~mm})$.

The peaks of the saddle (convexities) are commonly placed in an anteroposterior direction with the valleys (concavities) placed in a lateral direction, but a $90^{\circ}$ rotated placement may also be applied in case of severe neck angulation. The peaks are placed just below or at the level of the renal arteries. Active fixation is provided by 4 pairs of hooks, which are attached to both proximal rings at the peaks and valleys. The body is available in diameters ranging from 21.5 to $34 \mathrm{~mm}$ for aortic vessel inner diameters of 17.5 to $31 \mathrm{~mm}$. The instructions for use (IFU) advise a neck length $\geq 15 \mathrm{~mm}$, infrarenal angulation $\leq 90^{\circ}$, and an oversizing range from $10 \%$ to $20 \%$ with regard to inner vessel wall diameter[18].

Image Acquisition ECG-gated CT scans were performed on an Aquilion 64 CT scanner (Toshiba Medical Systems Corporation, Tokyo, Japan) or on a Somatom Definition Flash CT scanner (Siemens Healthineers, Forchheim, Germany) with a standardized low-dose scan protocol based on the routine static protocol for the abdomen. The 24-month scans were exclusively acquired on the Somatom Flash scanner. The scans were performed without contrast administration to preclude nephrotoxic effects. Scan parameters were as follows: rotation time 0.4 seconds (Aquilion), 0.3 seconds (Flash); collimation $64 \times 0.5 \mathrm{~mm}$ (Aquilion), $2 \times 128 \times 0.6 \mathrm{~mm}$ (Flash); slice thickness $1 \mathrm{~mm}$; slice increment $0.5 \mathrm{~mm}$; reconstructed matrix size $512 \times 512$ pixels, resulting in submillimeter isotropic datasets. The pitch factor was set automatically based on the heart rate. Tube voltage was set to $120 \mathrm{kV}$ with a tube current time product of 40,60 , or $80 \mathrm{~mA} . \mathrm{s}$ based on the patient's body mass index $(<20,20-25$, $>25 \mathrm{~kg} / \mathrm{m}^{2}$, respectively), since automated tube current modulation had to be turned off for ECG tracking. This resulted in a dose length product of $962.1 \pm 220.1 \mathrm{mGy} \cdot \mathrm{cm}$ for a scan length of $\sim 30 \mathrm{~cm}$. Images were acquired during a single breath hold after performing a standard breathing exercise. Retrospective gating was applied to obtain 10 equidistant volumes covering the cardiac cycle.

Image Processing The image processing steps included obtaining a phase- averaged 3-dimensional (3D) volume and segmentation of the 2 proximal sealing rings of the Anaconda stent-graft. Because a low-dose protocol for ECG-gated CT data was used, the exposure dose per reconstructed phase was decreased in comparison to a static CT scan, resulting in lower signal-to-noise (SNR) reconstructions. Since averaging the individual phases would result in a $3 \mathrm{D}$ volume that was subject to motion artifacts, a nonrigid B-spline registration was applied to obtain motion-compensated, time-averaged 3D volumes with improved SNR. A previously described registration algorithm[19,20] that was adjusted and validated for the purpose of stent-graft analysis in ECG-gated CT data[21] was used.

The time-averaged 3D volumes, which represented mid cardiac cycle, were used for segmentation of the dual ring and evaluation of the aortic vessel. Geometric models of the dual ring were obtained by applying a segmentation algorithm that was designed for stent analysis in volumetric CT data[22]. This 3-step segmentation algorithm used a minimum cost path (MCP) method to create a graph consisting of nodes and edges, where the edges represent the wire frame and the nodes are placed 
on the edges at wire crossings. In short, seed points that are likely to be on the wire frame of the stent-graft are detected (step 1), after which the MCP algorithm connects these seed points by tracing low-cost paths (step 2), that is, short paths between seed points through high-intensity voxels, resulting in a graph consisting of nodes that are connected by edges. Finally, because many of the traced edges do not fully run on the wire frame, an iterative cleaning operation (step 3) was performed to remove false edges and preserve only those that run through the middle of the wire bundle.

The algorithm was adjusted to allow for manual placement of additional seeds in order to prevent errors in the graph at the level of 2 high-intensity radiopaque markers on the hook struts. Further, a modification was made to allow for interactive restoration of edges in the graph that were falsely removed by the algorithm. False removal occurred in some cases with prominent intensity differences in the CT data between the first ring, the second ring, and the hooks. Finally, 1D quadratic polynomial fits in the $x, y$, and $z$ directions were implemented to obtain subvoxel positions. All segmentations were visually inspected in 3D maximum intensity projections.

Analysis The evolution over time of the size and shape of the proximal sealing rings was evaluated by measuring the diametric distances between the peaks $\left(d_{\text {peaks }}\right)$ and the valleys $\left(d_{\text {valleys }}\right)$ of the saddle-shaped rings in the segmented models through 24 months (Figure 1). The positions of the peaks and valleys on R1 and R2 were obtained as the midpoints between the nodes at each of the 4 hook pair crossings with R1 and R2. Ring diameter was calculated as the mean of $d_{\text {peaks }}$ and $d_{\text {valleys }}($ Equation 1). In addition to ring diameter, the degree of ring expansion was calculated as a percentage of the ring diameter divided by the predetermined flat ring diameter, that is, the nominal diameter as provided by the manufacturer (Equation 2). To evaluate changes in the shape of the rings, an asymmetry ratio was calculated as the maximum to minimum diametric distances between the peaks and valleys (Equation 3). For the purpose of visualizing the direction of asymmetry, the asymmetry ratio was also calculated by dividing $d_{\text {peaks }}$ by $d_{\text {valleys }}$. Additionally, the difference between the diametric distances was evaluated during follow-up.

$$
\begin{aligned}
\text { Ring diameter } & =\frac{d_{\text {peaks }}+d_{\text {valleys }}}{2} \\
\text { Ring expansion percentage } & =\frac{\text { ring diameter }}{\text { nominal ring diameter }} \times 100 \\
\text { Asymmetry ratio } & =\frac{\max \left(d_{\text {peaks }}, \quad d_{\text {valleys }}\right)}{\min \left(d_{\text {peaks }}, \quad d_{\text {valleys }}\right)}
\end{aligned}
$$

Statistical analysis Normality checks were performed to assess the distribution of the data, which are presented as means \pm standard deviation (range) for normally distributed continuous variables and as numbers for categorical variables. The median (interquartile range, IQR) is also given for non-parametric data.

Parametric data were compared between time points by use of a one-way analysis of variance (ANOVA) for repeated measures. For nonparametric data, the Friedman test was used instead with post hoc analysis using the Wilcoxon signed-rank test. The difference between diametric peak and valley distances was also compared at 
each time point for all patients in follow-up by using the Student $t$ test for paired data. Test results are presented with the $95 \%$ confidence interval (CI). Statistical significance was assumed when $\mathrm{p}<0.05$. A Bonferroni-adjusted significance level of $\mathrm{p}<0.01$ was used for nonparametric data. Statistical analysis was performed using SPSS Statistics (version 24.0; IBM Corporation, Armonk, NY, USA).

\section{Results}

No aneurysm-, device-, or procedure-related adverse events were reported through the 24-month follow-up in 13 of the 15 patients [ 1 patient died (carcinoma) and 1 patient withdrew]. The mean aneurysm sac diameter decreased from $60 \pm 7 \mathrm{~mm}$ at discharge to $44 \pm 12 \mathrm{~mm}$ after 24 months, with at least 5-mm sac diameter regression in 10 patients.

Evolution of the proximal rings Figure 3 presents the change in size of the dual rings from discharge to 24 months, as both the ring stent diameters and a percentage of their nominal size, that is, postdeployment ring expansion. For all patients, the diameter of both ring stents increased over time. From discharge to 24 months after EVAR, the mean ring diameter increased significantly by $2.2 \pm 1.0 \mathrm{~mm}(95 \%$ CI 1.2 to $3.2, \mathrm{p}<0.001)$ for $\mathrm{R} 1$ and $2.7 \pm 1.1 \mathrm{~mm}(95 \%$ CI 1.7 to 3.8. $\mathrm{p}<0.001)$ for R2 (Table 2). The maximum increase in ring diameter at 24 months was $5.0 \mathrm{~mm}(23 \%)$; however, the maximum increase in diametric distance $\left(d_{\text {peaks }}\right.$ or $\left.d_{\text {valleys }}\right)$ was $7.7 \mathrm{~mm}(34 \%)$ for a patient with an initial asymmetry ratio of 1.5 . The mean ring diameter increased most between discharge and 1 month for both rings, with a mean difference of $1.1 \pm 0.8 \mathrm{~mm}$ (95\% CI 0.3 to $1.8, \mathrm{p}=0.003)$ for $\mathrm{R} 1$ and $1.1 \pm 0.8 \mathrm{~mm}$ (95\% CI 0.3 to $1.8, \mathrm{p}=0.004$ ) for R2. Through 24 months, the mean percentage of ring expansion increased significantly by $7.6 \% \pm 3.8 \%(95 \%$ CI $4.0 \%$ to $11.2 \%, \mathrm{p}<0.001)$ for $\mathrm{R} 1$ and by $9.4 \% \pm 4.1 \%$ (95\% CI $5.5 \%$ to $13.3 \%, \mathrm{p}<0.001)$ for $\mathrm{R} 2$. The percentage increase was greatest during the first month for both ring stents $(\mathrm{p}=0.005)$ and highest in the 3 patients with the most pronounced saddle shapes because of greater oversizing $(33 \%, 40 \%$, and $47 \%)$. At 6 months, the rings had significantly expanded to a mean level of $96.6 \% \pm 2.1 \%$ for R1 and $94.8 \% \pm 2.7 \%$ for R2. After 6 months, the expansion percentage increased slowly, and the ring diameters stabilized close to their nominal size irrespective of the initial oversizing (98.3\% $\pm 1.1 \%$ for R1 and $97.2 \% \pm 1.4 \%$ for R2 at 24 months).

Figure 4 presents the evolution of ring shape for each individual patient, showing the asymmetry ratio of $d_{\text {peaks }}$ to $d_{\text {valleys }}$. At discharge, this ratio had a broad range of 0.65 to 1.21 for $\mathrm{R} 1$ and 0.72 to 1.50 for R2, but at 24 months, this range had narrowed to 0.94 to 1.11 for $\mathrm{R} 1$ and 0.94 to 1.24 for R2, meaning that the oval-shaped rings had adapted to be more circular. For R1, the average asymmetry ratio did not significantly change from discharge to 24 months $(\mathrm{p}=0.079)$, but a significant difference was found for $\mathrm{R} 2(\mathrm{p}=0.009$; Table 2$)$. The difference between the diametric peak and valleys distances was significant $(\mathrm{p}<0.005)$ at all time points for both rings, yet compared with discharge this difference had become smaller after 24 months (R1: median 2.0 vs $1.2 \mathrm{~mm}, \mathrm{p}=0.191$; $\mathrm{R} 2$ : median 2.8 vs $0.8 \mathrm{~mm}, \mathrm{p}=0.013$ ).

In Figure 5, the evolution of ring stent shape through 24 months is visualized for a patient with a pronounced saddle- shaped dual ring at discharge, showing the 

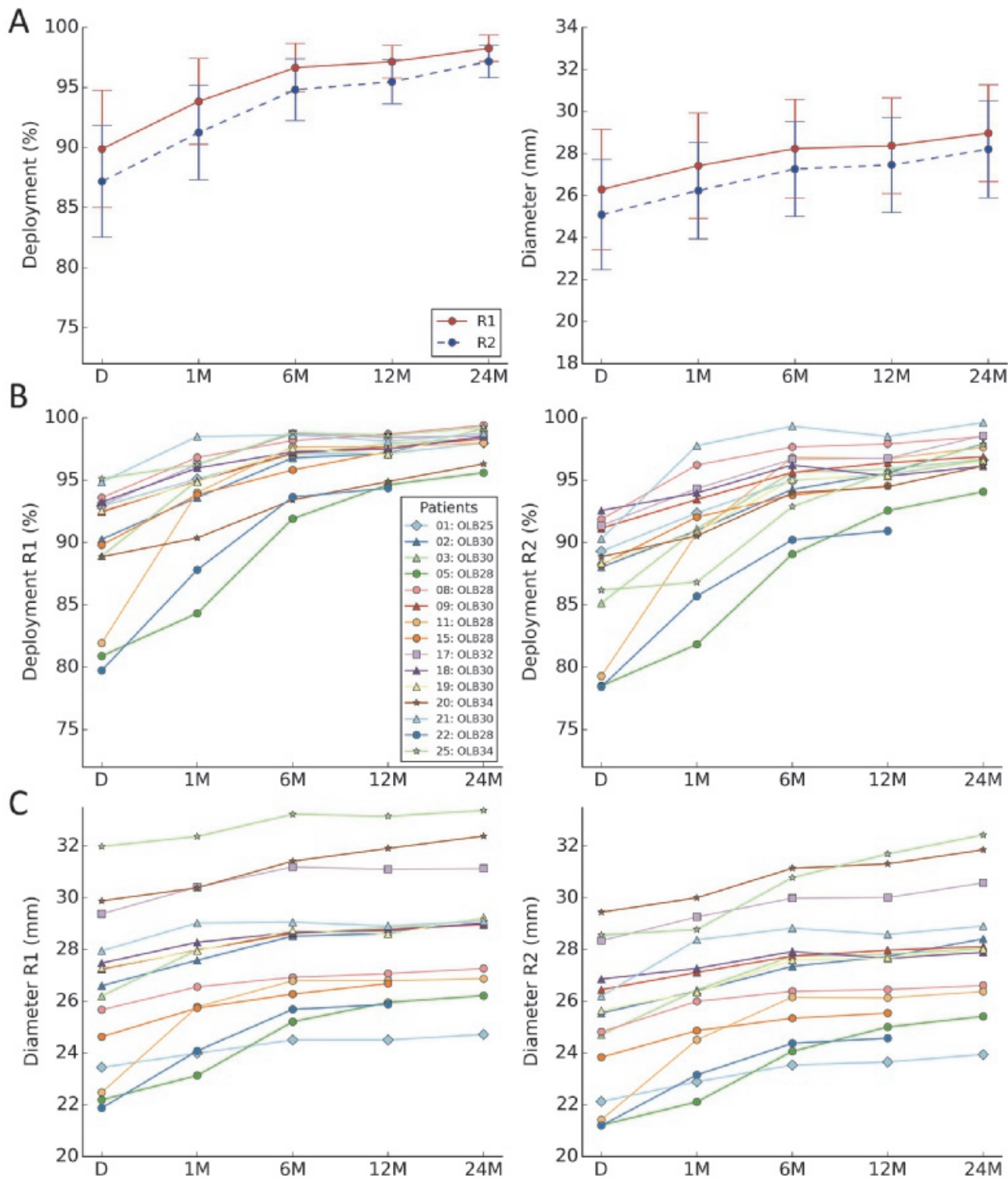

Figure 3. Evolution of the proximal dual ring of the Anaconda stent-graft from discharge to 24 months after endovascular aneurysm repair (EVAR), presented as the mean (dot) and standard deviation (whiskers) of the (A) expansion percentage (diameter ring / nominal diameter ring $\times 100)$ and the ring diameter $\left[\left(d_{\text {peaks }}+d_{\text {valleys }}\right) / 2\right]$ for both rings and $(\mathrm{B}$, C) for each individual patient for both rings. D, discharge; M, months after EVAR; OLB, main body device size; R1, first ring stent; R2, second ring stent. 
Table 2. Evolution of the size and shape of the proximal sealing rings through the 24-month follow-up. ${ }^{a}$

\begin{tabular}{|c|c|c|c|c|c|}
\hline & $\begin{array}{c}\text { Discharge } \\
(\mathrm{n}=15)\end{array}$ & $\begin{array}{c}1 \text { Month } \\
(\mathrm{n}=15)\end{array}$ & $\begin{array}{c}6 \text { Months } \\
(\mathrm{n}=15)\end{array}$ & $\begin{array}{l}12 \text { Months } \\
(\mathrm{n}=15)\end{array}$ & $\begin{array}{l}24 \text { Months } \\
(\mathrm{n}=13)\end{array}$ \\
\hline \multirow{3}{*}{$\begin{array}{l}\text { Expansion } \\
\mathrm{R} 1,{ }^{b} \%\end{array}$} & $89.9 \pm 5.1$ & $93.8 \pm 3.7$ & $96.6 \pm 2.1$ & $97.1 \pm 1.4$ & $98.3 \pm 1.1$ \\
\hline & $(79.7-95.1)$ & $(84.3-98.5)$ & (91.9-98.8) & $(94.4-98.7)$ & $(95.6-99.4)$ \\
\hline & - & $\mathrm{p}=0.005$ & $\mathrm{p}<0.001$ & $\mathrm{p}<0.001$ & $\mathrm{p}<0.001$ \\
\hline \multirow{3}{*}{$\begin{array}{l}\text { Expansion } \\
\mathrm{R} 2,^{b} \%\end{array}$} & $87.2 \pm 4.8$ & $91.2 \pm 4.1$ & $94.8 \pm 2.7$ & $95.5 \pm 1.9$ & $97.2 \pm 1.4$ \\
\hline & $(78.4-92.6)$ & (81.8-97.8) & (89.1-99.3) & $(90.9-98.5)$ & $(94.1-99.6)$ \\
\hline & - & $\mathrm{p}=0.005$ & $\mathrm{p}<0.001$ & $\mathrm{p}<0.001$ & $\mathrm{p}<0.001$ \\
\hline \multirow{3}{*}{$\begin{array}{l}\text { Diameter } \\
\text { change } \\
\mathrm{R} 1,^{c} \mathrm{~mm}\end{array}$} & - & $1.1 \pm 0.8$ & $1.9 \pm 1.0$ & $2.1 \pm 1.1$ & $2.2 \pm 1.0$ \\
\hline & & $(0.4-3.3)$ & $(1.1-4.3)$ & $(1.0-4.3)$ & $(1.2-4.4)$ \\
\hline & & $\mathrm{p}=0.003$ & $\mathrm{p}<0.001$ & $\mathrm{p}<0.001$ & $\mathrm{p}<0.001$ \\
\hline \multirow{3}{*}{$\begin{array}{l}\text { Diameter } \\
\text { change } \\
\mathrm{R} 2,^{c} \mathrm{~mm}\end{array}$} & - & $1.1 \pm 0.8$ & $2.2 \pm 1.0$ & $2.4 \pm 1.1$ & $2.7 \pm 1.1$ \\
\hline & & $(0.2-3.1)$ & $(1.1-4.7)$ & $(0.8-4.7)$ & $(1.0-5.0)$ \\
\hline & & $\mathrm{p}=0.004$ & $\mathrm{p}<0.001$ & $\mathrm{p}<0.001$ & $\mathrm{p}<0.001$ \\
\hline \multirow{5}{*}{$\begin{array}{l}\text { Asymmetry } \\
\text { ratio } \mathrm{R} 1^{d}\end{array}$} & $1.12 \pm 0.14$ & $1.10 \pm 0.11$ & $1.09 \pm 0.07$ & $1.07 \pm 0.07$ & $1.05 \pm 0.03$ \\
\hline & $(1.02-1.55)$ & $(1.01-1.40)$ & $(1.01-1.31)$ & $(1.00-1.27)$ & $(1.00-1.11)$ \\
\hline & 1.07 & 1.07 & 1.08 & 1.06 & 1.04 \\
\hline & {$[1.03,1.16]$} & {$[1.01,1.15]$} & {$[1.04,1.09]$} & {$[1.03,1.09]$} & {$[1.03,1.08]$} \\
\hline & - & $\mathrm{p}=0.430$ & $\mathrm{p}=0.236$ & $\mathrm{p}=0.058$ & $\mathrm{p}=0.079$ \\
\hline \multirow{5}{*}{$\begin{array}{l}\text { Asymmetry } \\
\text { ratio } 2^{d}\end{array}$} & $1.17 \pm 0.16$ & $1.14 \pm 0.14$ & $1.10 \pm 0.09$ & $1.08 \pm 0.07$ & $1.06 \pm 0.07$ \\
\hline & $(1.02-1.50)$ & $(1.02-1.48)$ & $(1.03-1.31)$ & $(1.00-1.24)$ & $(1.00-1.24)$ \\
\hline & 1.12 & 1.09 & 1.07 & 1.06 & 1.03 \\
\hline & {$[1.04,1.24]$} & {$[1.04,1.17]$} & {$[1.04,1.12]$} & {$[1.03,1.12]$} & {$[1.02,1.10]$} \\
\hline & - & $\mathrm{p}=0.146$ & $\mathrm{p}=0.027$ & $\mathrm{p}=0.010$ & $\mathrm{p}=0.009$ \\
\hline \multirow{5}{*}{$\begin{array}{l}\text { Difference } \\
\mathrm{d}_{\text {peaks }}- \\
\mathrm{d}_{\text {valleys }} \\
\mathrm{R} 1, \mathrm{~mm}\end{array}$} & $2.7 \pm 2.7$ & $2.4 \pm 2.5$ & $2.2 \pm 1.7$ & $1.9 \pm 1.6$ & $1.5 \pm 1.0$ \\
\hline & $(0.4-10.2)$ & $(0.2-8.3)$ & $(0.2-6.9)$ & $(0.0-6.1)$ & $(0.1-3.2)$ \\
\hline & 2.0 & 2.1 & 1.9 & 1.6 & 1.2 \\
\hline & {$[0.6,4.4]$} & {$[0.2,4.0]$} & {$[1.1,2.5]$} & {$[0.8,2.5]$} & {$[0.8,2.2]$} \\
\hline & - & $\mathrm{p}=0.635$ & $\mathrm{p}=0.331$ & $\mathrm{p}=0.131$ & $\mathrm{p}=0.191$ \\
\hline \multirow{5}{*}{$\begin{array}{l}\text { Difference } \\
\mathrm{d}_{\text {peaks }}- \\
\mathrm{d}_{\text {valleys }} \\
\mathrm{R} 2, \mathrm{~mm}\end{array}$} & $3.6 \pm 3.3$ & $3.2 \pm 3.0$ & $2.6 \pm 2.1$ & $2.2 \pm 1.8$ & $1.7 \pm 1.7$ \\
\hline & $(0.4-11.2)$ & $(0.5-10.8)$ & $(0.8-7.3)$ & $(0.6-6.8)$ & $(0.1-5.8)$ \\
\hline & 2.8 & 2.4 & 2.0 & 1.5 & 0.8 \\
\hline & {$[1.0,5.4]$} & {$[0.8,4.0]$} & {$[1.0,3.2]$} & {$[0.8,3.0]$} & {$[0.4,2.6]$} \\
\hline & - & $\mathrm{p}=0.366$ & $\mathrm{p}=0.046$ & $\mathrm{p}=0.013$ & $\mathrm{p}=0.013$ \\
\hline
\end{tabular}

Abbreviations: R1, first ring stent; R2, second ring stent.

${ }^{a}$ Data are presented as the means \pm standard deviation (range) and median [interquartile range Q1, Q3] as applicable. $\mathrm{P}$ values refer to discharge vs other time points.

${ }^{b}$ Expansion percentage $=($ diameter $/$ nominal diameter $) \times 100$.

${ }^{c}$ Diameter $=\left(\mathrm{d}_{\text {peaks }}+\mathrm{d}_{\text {valleys }}\right) / 2$.

${ }^{d}$ Asymmetry ratio $=\max \left(\mathrm{d}_{\text {peaks }}, \mathrm{d}_{\text {valleys }}\right) / \min \left(\mathrm{d}_{\text {peaks }}, \mathrm{d}_{\text {valleys }}\right)$.

adaptation from an asymmetric saddle to flattened symmetric ring stents. Note that in this case the orientation of ring stent asymmetry changed during the first month. In this patient, the diameter of the aneurysm sac decreased from $65 \mathrm{~mm}$ at discharge to $37 \mathrm{~mm}$ after 24 months. 

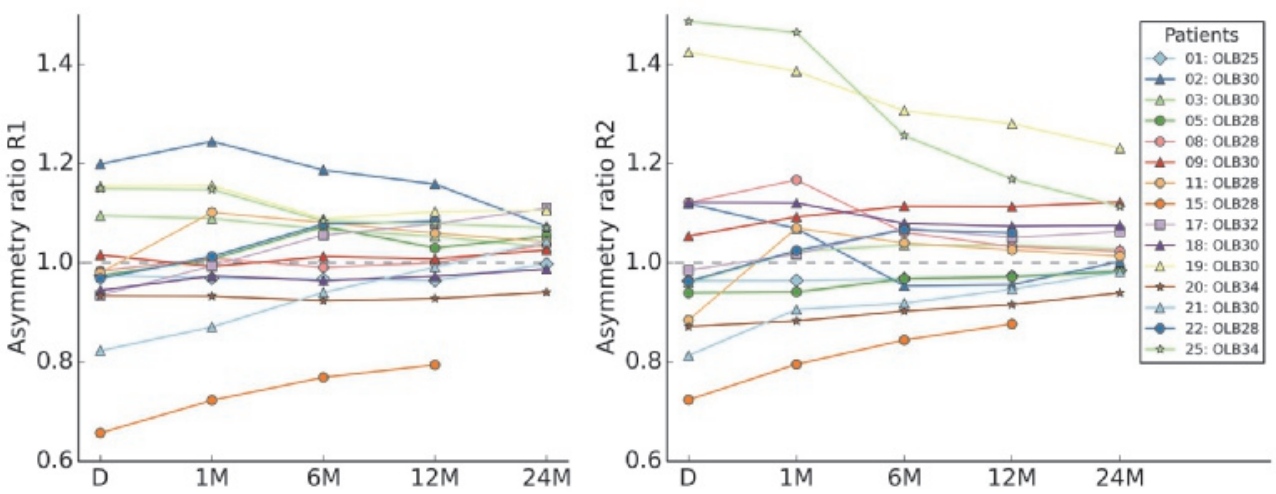

Figure 4. Evolution of the asymmetry ratio of the proximal dual ring of the Anaconda stent-graft from discharge to 24 months after endovascular aneurysm repair (EVAR). Here, for the purpose of visualizing the direction of asymmetry, the asymmetry ratio of each ring was calculated as $d_{\text {peaks }}$ to $d_{\text {valleys }}$ at a given time. The dashed line is a ratio of 1.0 , which represents symmetric ring dimensions. In 2 cases (\#19 and \#25), the body was positioned with $90^{\circ}$ rotation (saddle peaks in lateral direction and valleys in anteroposterior direction). D, discharge; M, months after EVAR; OLB, main body device size; R1, first ring stent; R2, second ring stent.

\section{Discussion}

In this study, substantial variation in the initial size of the dual rings was observed per patient and per ring stent, though the first ring had consistently expanded further compared to the second ring. Interestingly, despite this initial variation, there was consistent expansion of the saddle- shaped rings to near nominal size irrespective of the initial degree of oversizing (Figure 3). Expansion of the rings occurred mostly within the first 6 months after EVAR, with the greatest degree of expansion during the first month and in patients with the most pronounced saddle shapes (greater oversizing). An explanation for this observation could be found in the stress-strain curve of nitinol; after release of the stent-graft from the delivery system, the force can be initially higher at higher deflection[23] and thus greater oversizing. Notably, in one of these patients the ring stents expanded rapidly within 1 month, while in the other 2 patients the saddle shape was preserved for a longer period of time (Figure 3). The reason for this could be differences in aortic wall characteristics (i.e., stiffness), since there were some calcifications at the level of the dual rings in the latter 2 patients.

Another important finding of this work is that during the course of ring expansion the oval-shaped ring stents con form symmetrically and become circular. This process may take $>2$ years when the initial shape is highly asymmetric (Figure 4). Specifically the 3 patients with the most marked infrarenal neck angulation $\left(>70^{\circ}\right)$ showed the highest degree of ring stent asymmetry (Figure 6). These results imply that over time the aortic neck deforms due to the radial force of the ring stents. Additionally, these adaptations of the aortic neck may have implications for the durability of the seal and fixation. However, clinical midterm data on the Anaconda shows that migration and 

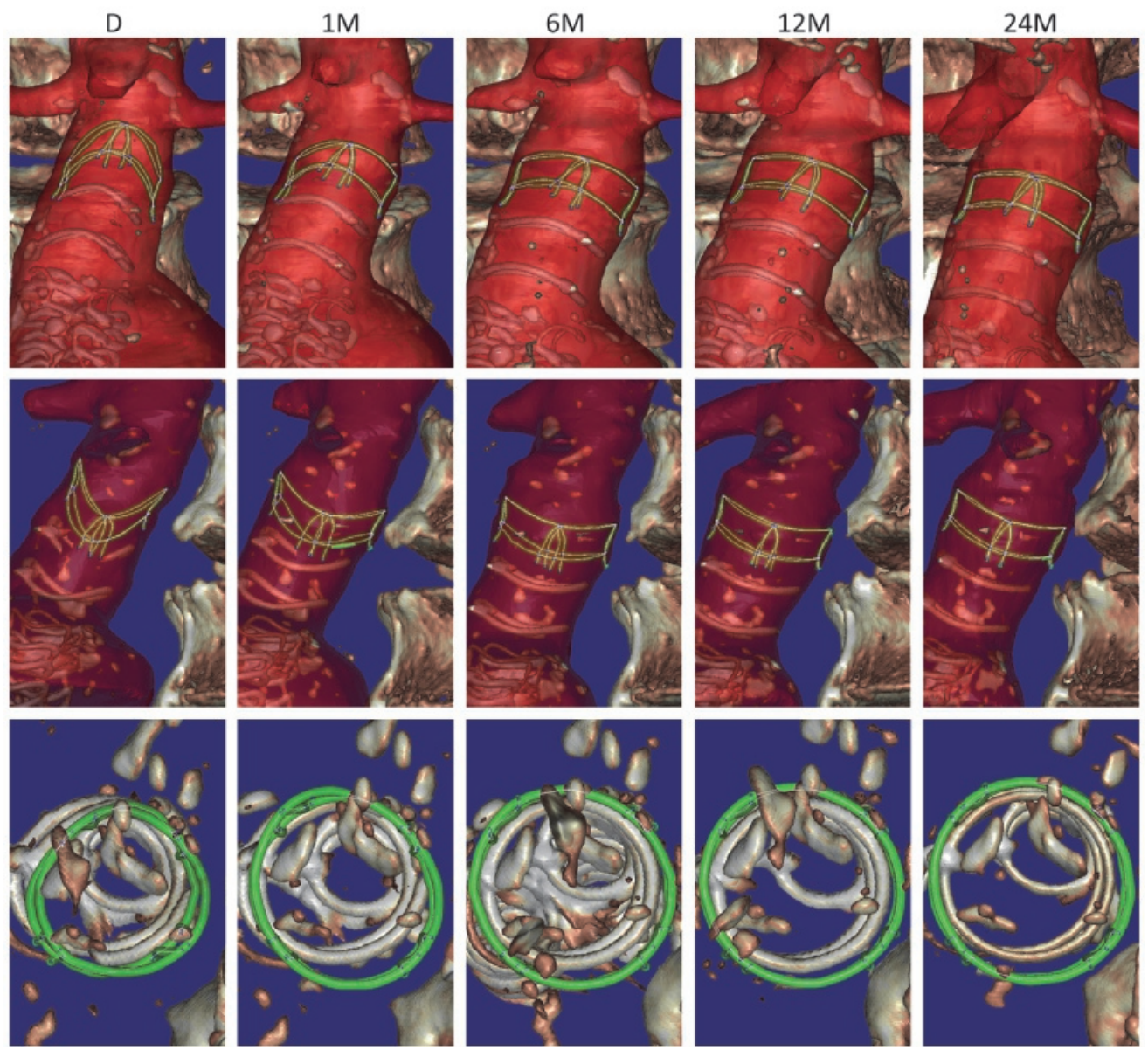

Figure 5. A clinical case (\#11) demonstrating the evolving adaptation of the proximal sealing and fixation rings from discharge to 24 months after endovascular aneurysm repair (EVAR). The illustration shows anterior to posterior views (top), lateral views from left to right (middle), and top views from superior to inferior (bottom) with the model rotated to be perpendicular to the screen. The model obtained by segmentation is shown in green, with the white lines and blue dots representing the edges and nodes in the model, respectively. The vertebrae, remaining part of the stent-graft, and calcifications are visualized as a surface rendering. A segmentation of the aortic vessel (outer wall), including the proximal part of the renal arteries and superior mesenteric artery, is shown in red. D, discharge; M, months after EVAR. 
endoleak rates are low [6-8], even in severely angulated proximal necks $[9,10]$.

Recently, the largest published single-center clinical experience using the Anaconda reported a $1.1 \%$ rate of late type Ia endoleak and no migration at a mean follow-up of $32.9 \pm 23.3$ months[7]. In our present cohort no clinical failures related to device migration or endoleak were observed, which is reflected in the regression of aneurysm sac diameters in the majority of patients. To our knowledge, no reports have been published on the symmetry of ring expansion in other self-expanding stent-grafts.

Because the ring stents continue to expand to near nominal size, the vessel wall is subjected to tensile stress and may undergo several millimeters of dilatation at the level of the sealing and fixation rings, depending on the degree of oversizing. Also, when asymmetric ring stents become circular, the degree of ring expansion over a single axis can be extensive $(>5 \mathrm{~mm})$. These significant levels of ring expansion may raise concerns related to aortic neck dilatation (AND), which has been associated with migration, endoleak, and increased reintervention rates[24-27]. However, ring expansion also enhances apposition between the graft and the vessel wall. Moreover, local dilatation due to ring expansion does not necessarily result in dilatation of the entire neck. In fact, ring expansion at only the sealing zone may prevent the stentgraft from migrating. In that sense, local proximal radial strength might be preferred over designs that have radial stents through the length of the device. Also, expansion of the rings seems to support embedding of the hooks into the vessel wall (Figure 5), which a few millimeters of migration can facilitate.

Nevertheless, it must be acknowledged that if the neck becomes diseased and subject to progressive AND, the dimensions of the neck could exceed the dimensions of the fully expanded stent-graft. In this case, an opening between the wall and the graft and/or migration may occur, resulting in type Ia endoleak. Our results suggest that after 6 months the ring stents have little remaining expansion capacity to adapt to potential progressive AND, while others have assumed that in case of progressive AND, the ring stents adapt and the saddle flattens[11,12].

Certainly, all self-expanding stent-grafts have the limitation that they will accommodate vessel dilatation only up to the point where it reaches their designed diameter. Of importance is whether dilatation of the aortic neck continues after the stent-graft has fully expanded. Monahan et al[28] investigated AND after implantation of the Zenith stent-graft and found that the neck dilates until the stent-graft has approximated its designed diameter. They found that the rate of neck expansion was greatest at early follow-up intervals (1-6 months). Moreover, they concluded that this dilatation is not associated with type I endoleak. Interestingly, 2 other studies that investigated self-expanding stent-grafts reported that AND occurred specifically within the first 6 months but then stabilized through 24 months[29,30]. Also Cao et al[24] concluded that AND is common at midterm follow-up but shows little tendency to progress at a mean follow-up of 18 months, although late reintervention was most frequently necessary in a small number of patients who developed severe ongoing AND. Although several studies do raise concern regarding continuing AND[4,31], these results imply that midterm AND is not necessarily a clinical problem and may be misinterpreted from the observation of stent-graft expansion. Moreover, the apparent absence of AND after treatment with balloon-expandable stent-grafts[32,33], 

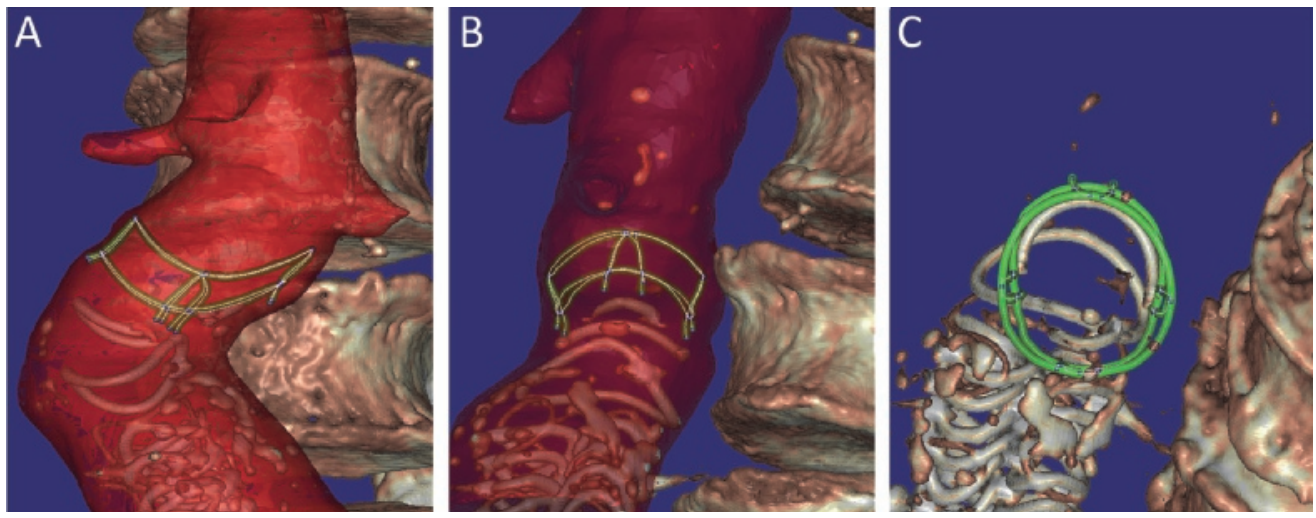

Figure 6. A clinical case (\#19) with a high degree $\left(>90^{\circ}\right)$ of infrarenal neck angulation (outside the instructions for use) demonstrating asymmetric ring dimensions at discharge in the (A) anteroposterior view, (B) lateral view, and (C) oblique craniocaudal view with the model of the dual ring perpendicular to the screen. The main body was positioned with $90^{\circ}$ rotation to allow the proximal rings and the anteroposteriorly positioned legs to better follow the course of the angulation. Both rings were not positioned in line with the aorta but rather inclined.

which do not exert continued radial force, supports this hypothesis.

The design of the Anaconda allows that the peaks of the proximal saddle-shaped ring can be placed at the level of or just above the renal artery origins, without actually covering these ostia with the valley of the ring. However, when the proximal rings expand, becoming more circular and the saddle shape flattens, the valleys of the proximal ring may come up a few millimeters, with the potential risk of covering the renal artery ostia[6,7]. Although renal artery occlusion due to upstream migration of the valleys seems to be rare, the clinical consequences can be substantial[8,9]. Whether the valleys indeed migrate upstream or whether the complete proximal device migrates further downstream is the subject of current investigation.

Finally, the evolution of ring stent expansion may differ per design. Also, the behavior of suprarenal fixating devices could differ from what has been observed in the present study, since the stiffness and thickness of the suprarenal and infrarenal vessel segments are different[34]. Therefore, future work should also study the behavior of suprarenally fixating devices, including fenestrated EVAR, especially since there is growing evidence that suprarenal fixation results in a slightly higher risk of renal complications [35].

In sum, it is of utmost importance to understand the sealing and fixation behavior of individual stent-graft designs to be able to recognize adaptations of the aortic neck that occur due to the fixation mechanism of the stent-graft itself, rather than disease progression, which may not have further clinical consequences. Additionally, knowledge of stent-graft-neck interactions for both infra- and suprarenal fixating stent-grafts is crucial for selection of the best device, size, and deployment technique based on each patient's aneurysm anatomy. 
Limitations A potential shortcoming of this study is the low number of patients. Nevertheless, because of the prospective design of the LSPEAS trial, follow-up examinations at 5 standardized time points through 24 months could be obtained. Even though the present number of patients is not enough to evaluate clinical outcome, it does allow for a detailed evaluation of stent-graft behavior. In addition, the results demonstrated clear trends and significant changes. Also, ECG-gated CT scans and advanced postprocessing using an algorithm specifically designed to analyze stents in volumetric CT data allowed accurate repeated measurements at the same time during the cardiac cycle, which is not possible with static CT scans.

\section{Conclusion}

This prospective study has provided insight into the evolution of the proximal sealing and fixation rings of the Anaconda AAA stent-graft system. The saddle-shaped rings radially expanded to near nominal size within 6 months after EVAR despite a broad range of initial oversizing, all without type I or III endoleak. Interestingly, the asymmetrically shaped ring stents conformed symmetrically and became nearly circular through 24 months. These observations imply that over time the aortic neck conforms to the size of the self-expanding nitinol rings irrespective of neck characteristics. It is therefore advisable to avoid excessive oversizing. Furthermore, one should be careful not to misinterpret neck dilatation due to ring expansion for disease progression while at the same time being alert if the neck continues to dilate after 6 to 12 months when the rings have expanded to their designed size. Local dilatation of the neck due to ring expansion is beneficial for the fixation and sealing as long as neck dilatation is localized exclusively to the sealing zone. Additional research is necessary to investigate potential dilatation of the entire neck and the relation with postoperative ring expansion.

\section{Acknowledgments}

The authors are grateful to Anja Stam (clinical research officer, Department of Surgery, Medisch Spectrum Twente) for her assistance in conducting the LSPEAS trial and to Bert Klein Rot (chief laboratory technician CT, Department of Radiology, Medisch Spectrum Twente) for his technical support with the CT acquisitions. Furthermore, the authors thank all vascular surgeons for including patients in the LSPEAS trial.

\section{Declaration of conflicting interests}

Robert H. Geelkerken is a consultant for Vascutek Ltd.

\section{Funding}

The LSPEAS trial (Trialregister.nl; identifier NTR4276) was supported in part by an unrestricted research grant from Vascutek Ltd, a TERUMO Company, and in part 
by the Dutch Ministry of Economic Affairs under TKI-Allowance under the TKIprogramme Life Sciences \& Health.

\section{References}

1. Smeds MR, Charlton-Ouw KM. Infrarenal endovascular aneurysm repair: New developments and decision making in 2016. Semin Vasc Surg. 2016;29:27-34.

2. Arko FR 3rd, Murphy EH, Boyes C, Nussbaum T, Lalka SG, Holleman J, et al. Current status of endovascular aneurysm repair: 20 years of learning. Semin Vasc Surg. 2012;25:131-5.

3. Antoniou GA, Georgiadis GS, Antoniou SA, Kuhan G, Murray D. A meta-analysis of outcomes of endovascular abdominal aortic aneurysm repair in patients with hostile and friendly neck anatomy. J Vasc Surg. 2013;57:527-38.

4. Diehm N, Dick F, Katzen BT, Schmidli J, Kalka C, Baumgartner I. Aortic neck dilatation after endovascular abdominal aortic aneurysm repair: A word of caution. J Vasc Surg. 2008;47:886-92.

5. Thomas B, Sanchez L. Proximal migration and endoleak: Impact of endograft design and deployment techniques. Semin Vasc Surg. 2009;22:201-6. 6. Rödel SGJ, Geelkerken RH, Prescott RJ, Florek HJ, Kasprzak P, Brunkwall J. The Anaconda AAA stent graft system: 2-year clinical and technical results of a multicentre clinical evaluation. Eur J Vasc Endovasc Surg. 2009;38:732-40.

7. Freyrie A, Gallitto E, Gargiulo M, Faggioli G, Massoni CB, Mascoli C, et al. Results of the endovascular abdominal aortic aneurysm repair using the Anaconda aortic endograft. J Vasc Surg. 2014;60:1132-9.

8. Saratzis N, Melas N, Saratzis A, Lazarides J, Ktenidis K, Tsakiliotis S, et al. Anaconda aortic stent-graft: single-center experience of a new commercially available device for abdominal aortic aneurysms. J Endovasc Ther. 2008;15:33-41.

9. Rödel SGJ, Zeebregts CJ, Huisman AB, Geelkerken RH. Results of the Anaconda endovascular graft in abdominal aortic aneurysm with a severe angulated infrarenal neck. J Vasc Surg. 2014;59:1495-1501.e1.

10. Freyrie A, Gallitto E, Gargiulo M, Mascoli C, Faggioli G, Pini R, et al. Proximal aortic neck angle does not affect early and late EVAR outcomes: an Anaconda ${ }^{\mathrm{TM}}$ Italian registry analysis. J Cardiovasc Surg (Torino). 2014;55:671-7.

11. Stella A, Freyrie A, Gargiulo M, Faggioli GL. The advantages of Anaconda endograft for AAA. J Cardiovasc Surg (Torino). 2009;50:145-52.

12. Freyrie A, Testi G, Faggioli GL, Gargiulo M, Giovanetti F, Serra C, et al. Ringstents supported infrarenal aortic endograft fits well in abdominal aortic aneurysms with tortuous anatomy. J Cardiovasc Surg (Torino). 2010;51:467-74.

13. Ahn SS, Rutherford RB, Johnston KW, May J, Veith FJ, Baker JD, et al. Reporting standards for infrarenal endovascular abdominal aortic aneurysm repair. J Vasc Surg. 1997;25:405-10. 14. Fitz-Henry J. The ASA classification and peri-operative risk. Ann R Coll Surg Engl. 2011;93:185-7.

15. Bungay P. The use of the Anaconda ${ }^{\mathrm{TM}}$ stent graft for abdominal aortic aneurysms. 
J Cardiovasc Surg (Torino). 2012;53:571-7.

16. Harris PL, Buth J, Mialhe C, Myhre HO, Norgren L. The need for clinical trials of endovascular abdominal aortic aneurysm stent-graft repair: The EUROSTAR project. J Endovasc Ther. 1997;4:72-7.

17. Balm R, Stokking R, Kaatee R, Blankensteijn JD, Eikelboom BC, Van Leeuwen MS. Computed tomographic angiographic imaging of abdominal aortic aneurysms: Implications for transfemoral endovascular aneurysm management. J Vasc Surg. 1997;26:231-7.

18. Terumo Aortic. Anaconda ${ }^{\text {TM }}$ AAA Stent Graft System: Combined IFU. Available from: http://www.vascutek.com/site/assets/files/3457/301179_combined_anaconda_one-lok_ifu.pdf. Accessed 29 March 2019.

19. Klein A, Kroon D-J, Hoogeveen Y, Schultze Kool LJ, Renema WKJ, Slump CH. Multimodal image registration by edge attraction and regularization using a B-spline grid. Proc SPIE Med Imaging. 2011;7962:796220-8.

20. Klein A. A tool for studying the motion of stent grafts in AAA. Segmentation and motion estimation of stent grafts in abdominal aortic aneurysms. [Enschede]: $\mathrm{PhD}$ Dissertation, University of Twente; 2011. p. 121-37.

21. Koenrades MA, Struijs EM, Klein A, Kuipers H, Geelkerken RH, Slump CH. Validation of an image registration and segmentation method to measure stent graft motion on ECG-gated CT using a physical dynamic stent graft model. Proc SPIE Med Imaging. 2017;10134:1013411-8.

22. Klein A, van der Vliet JA, Oostveen LJ, Hoogeveen Y, Schultze Kool LJ, Renema WKJ, et al. Automatic segmentation of the wire frame of stent grafts from CT data. Med Image Anal. 2012;16:127-39.

23. Stockel D. Nitinol - A material with unusual properties. Endovasc Updat. $1998 ; 1-9$.

24. Cao P, Verzini F, Parlani G, De Rango P, Parente B, Giordano G, et al. Predictive factors and clinical consequences of proximal aortic neck dilatation in 230 patients undergoing abdominal aorta aneurysm repair with self-expandable stent-grafts. J Vasc Surg. 2003;37:1200-5.

25. Dalainas I, Nano G, Bianchi P, Ramponi F, Casana R, Malacrida G, et al. Aortic neck dilatation and endograft migration are correlated with self-expanding endografts. J Endovasc Ther. 2007;14:318-23.

26. Dillavou ED, Muluk S, Makaroun MS. Is neck dilatation after endovascular aneurysm repair graft dependent? Results of 4 US Phase II trials. Vasc Endovascular Surg. 2005;39:47-54.

27. Oberhuber A, Buecken M, Hoffmann M, Orend K-HH, Mhling BM, Mühling BM. Comparison of aortic neck dilatation after open and endovascular repair of abdominal aortic aneurysm. J Vasc Surg. 2012;55:929-34.

28. Monahan TS, Chuter TAM, Reilly LM, Rapp JH, Hiramoto JS. Long-term follow-up of neck expansion after endovascular aortic aneurysm repair. J Vasc Surg. 2010;52:303-7. 
29. Soberón AB, de Garcia MM, Möll GG, Vigil BR, Krauel MA, Walter RA-S. Follow-up of aneurysm neck diameter after endovascular repair of abdominal aortic aneurysms. Ann Vasc Surg. 2008;22:559-63.

30. Sternbergh WC, Money SR, Greenberg RK, Chuter TAM, Moore WS, Buth J, et al. Influence of endograft oversizing on device migration, endoleak, aneurysm shrinkage, and aortic neck dilation: Results from the Zenith multicenter trial. J Vasc Surg. 2004;39:20-6.

31. Filis KA, Galyfos G, Sigala F, Tsioufis K, Tsagos I, Karantzikos G, et al. Proximal aortic neck progression: Before and after abdominal aortic aneurysm treatment. Front Surg. 2017;4:1-6.

32. Malas MB, Ohki T, Veith FJ, Chen T, Lipsitz EC, Shah AR, et al. Absence of proximal neck dilatation and graft migration after endovascular aneurysm repair with balloon-expandable stent-based endografts. J Vasc Surg. 2005;42:639-44.

33. Peirano MAM, Bertoni HG, Chikiar DS, Martínez JMP, Girella GA, Barone HD, et al. Size of the proximal neck in AAAs treated with balloon-expandable stent-grafts: CTA findings in mid-to long-term follow-up. J Endovasc Ther. 2009;16:696-707.

34. Wolinsky H. Comparison of medial growth of human thoracic and abdominal aortas. Circ Res. 1970;27:531-8.

35. Zettervall SL, Soden PA, Deery SE, Ultee K, Shean KE, Shuja F, et al. Comparison of renal complications between endografts with suprarenal and infrarenal fixation. Eur J Vasc Endovasc Surg. 2017;54:5-11. 





\section{Chapter 3}

\section{Geometric remodeling of the perirenal aortic neck at and adjacent to the double sealing ring of the Anaconda stent-graft after endovascular aneurysm repair}

Maaike A. Koenrades ${ }^{a, b, c}$, Marianne R.F. Bosscher ${ }^{b}$, Jouke T. Ubbink ${ }^{d}$, Cornelis H. Slump ${ }^{c}$, and Robert H. Geelkerken ${ }^{a, b}$

${ }^{a}$ Multi-modality Medical Imaging (M3I) group, Faculty of Science and Technology, Technical Medical Centre, University of Twente, Enschede, the Netherlands

${ }^{b}$ Department of Vascular Surgery, Medisch Spectrum Twente, Enschede, the Netherlands

${ }^{c}$ Robotics and Mechatronics (RaM) group, Faculty of Electrical Engineering, Mathematics and Computer Science, Technical Medical Centre, University of Twente, Enschede, the Netherlands

${ }^{d}$ Technical Medicine, Faculty of Science and Technology, Technical Medical Centre, University of Twente, Enschede, the Netherlands

Preliminary data from this study were presented at LINC (January 30 - February 2, 2018; Leipzig, Germany) 


\section{Abstract}

Purpose: To evaluate if the radial force of the double sealing ring of the Anaconda stent-graft induces dilatation in the perirenal aortic neck adjacent to the rings.

Methods: This study evaluated the serial electrocardiogram-gated computed tomography scans of 15 abdominal aortic aneurysm patients (mean age $72.8 \pm 3.7$ years; 14 men) who were treated electively using an Anaconda stent-graft. Follow-up scans were conducted before discharge and at 1, 6, 12, and 24 months after endovascular repair. Diameter and area were assessed perpendicular to the aortic centerline along the perirenal aortic neck, which was subdivided into 3 zones: the suprastent, the stent, and the infrastent zones. Measurements were performed independently by 2 experienced observers using dedicated 3-dimensional image processing software.

Results: Between discharge and 2 years follow-up the diameter and area remained stable in the suprastent zone [average diameter change: $-0.1 \pm 0.4 \mathrm{~mm}(-0.4 \% \pm 1.7 \%)$, $\mathrm{p}=0.893$; average area change: $\left.-2.9 \pm 17.2 \mathrm{~mm}^{2}(-0.7 \% \pm 3.4 \%), \mathrm{p}=0.946\right]$, increased in the stent zone [average diameter change: $+1.9 \pm 1.0 \mathrm{~mm}(+7.3 \% \pm 4.0 \%), \mathrm{p}<0.001$; average area change: $\left.+84.3 \pm 48.3 \mathrm{~mm}^{2}(+15.5 \% \pm 8.7 \%), \mathrm{p}<0.001\right]$, and diverged in the infrastent zone [average diameter change: $-0.8 \pm 2.2 \mathrm{~mm}(-2.3 \% \pm 7.4 \%), \mathrm{p}>0.99$; average area change: $-34.6 \pm 102.3 \mathrm{~mm}^{2}(-4.1 \% \pm 14.8 \%), \mathrm{p}>0.99$; increased in 4 patients, decreased in 9 patients].

Conclusion: After Anaconda implantation the infrarenal aortic neck accommodated to the expansion of the sealing rings at the stent zone. Below the stent zone the neck diameter decreased in the majority of patients, while an increase was related to downstream displacement of the main body. A decrease in size in the infrastent zone may contribute to durable sealing and fixation. A personalized follow-up scheme based on geometric neck remodeling should be feasible if our observations are confirmed in larger, long-term studies.

Keywords: Abdominal aortic aneurysm, aortic neck dilatation, endograft, endovascular aneurysm repair, fixation, geometry, neck remodeling, sealing rings, stent-graft 


\section{Introduction}

Aortic neck dilatation (AND) after endovascular aneurysm repair (EVAR) is a major concern for the durability of an effective proximal seal between the stent-graft and the aortic wall[1-3]. AND appeared to be present in nearly $25 \%$ of EVAR patients according to a recent pooled analysis[2]. Controversy surrounds the cause and clinical relevance of $\mathrm{AND}[1-3]$, especially since this phenomenon is seen with both open and endovascular repair of abdominal aortic aneurysm (AAA)[4]. AND after EVAR has been associated with migration and type Ia endoleak[5-9], which encompass the most common reasons for reinterventions[10-12], though others did not show this relation[13-16]. Oversizing of self-expanding stent-grafts may to some extent be the reason for AND[2]. Additionally, the evolution of the aortic neck may differ per stent-graft design depending on the sealing and fixation properties.

The Anaconda AAA stent-graft system (Terumo Aortic, Inchinnan, Scotland, UK) differs from most other devices in its proximal dual rings for sealing and fixation. Once deployed in the infrarenal neck, the 2 nitinol stent-rings assume the shape of a saddle, with peaks and valleys as a result of compression against the aortic wall. The stent-rings exert a continuing outward radial force on the aortic wall, which has been shown to result in proximal ring expansion to near-nominal size during the first 6 to 12 months after EVAR, irrespective of oversize[17]. Recently, Vukovic et al[18] confirmed this finding, reporting significant proximal landing zone dilatation after EVAR using the Anaconda. Still, it remained unclear whether the dilatation was localized at the level of the sealing rings, leaving the remaining portion of the neck unaffected, or whether the complete neck may have been affected. Such differentiation is imperative to understand the clinical significance of AND and to be able to identify patients at risk of migration and type Ia endoleak.

In the present study, we continue analysis of a previously reported patient cohort[17] to seek full understanding of abdominal aortic neck remodeling after Anaconda stent-graft implantation by investigating the geometric evolution of the entire perirenal neck segment above, at, and below the 2 fixation and sealing rings.

\section{Methods}

Study design and patient sample The present study evaluated 15 asymptomatic patients (mean age $72.8 \pm 3.7$ years; 14 men) with an infrarenal AAA who underwent elective EVAR between April 2014 and May 2015 with an Anaconda AAA stent-graft and had at least 12 months of imaging follow-up. Details of the patient sample, including preoperative anatomical characteristics by standard computed tomography angiography (CTA) imaging, and the image acquisition protocol were reported in a prior publication about the evolution of the Anaconda proximal sealing rings[17].

Sizes of the stent-graft body ranged from 25.5 to $34 \mathrm{~mm}$. The device was oversized by $17 \%$ to $47 \%$ (mean $31 \%$ ) based on inner wall diameters from static preoperative CTA scans. (In our practice, oversize was substantially increased particularly in case of unfavorable neck anatomy to increase the adaptive capacity of the sealing rings and intentional non-perpendicular placement with respect to the flow axis in angulated necks.) 


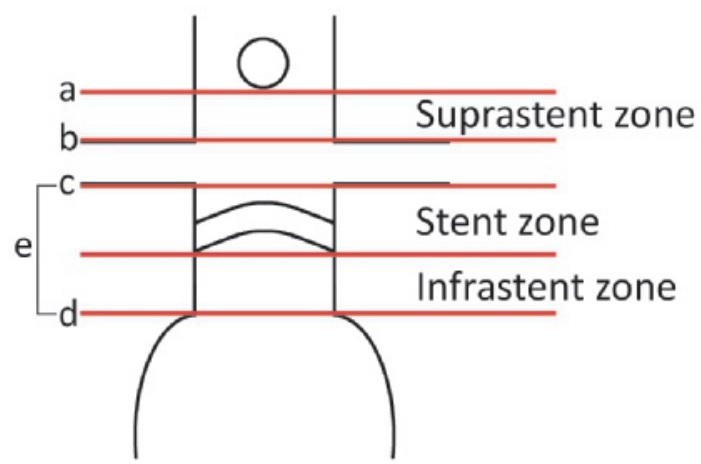

Figure 1. Subdivision of measurements (a-e) into 3 zones: (1) suprastent zone, (2) stent zone, (3) infrastent zone.

Patients were followed according to study protocol for 2 years after EVAR by noncontrast electrocardiogram (ECG)-gated CT scans before discharge and after 1, 6, 12, and 24 months of follow-up. The predischarge scans were conducted within 3 days after the EVAR procedure. Duplex ultrasound examinations were complementary to the noncontrast CT scans to follow the exclusion of the aneurysm. After 2 years, patients were followed by duplex ultrasound examinations and plain radiography according to standard practice.

The patient data were prospectively collected in a database registered on Trialregister.nl (NTR4276). The study protocol was approved by the institutional review board of the Medisch Spectrum Twente. Written informed consent was obtained for each subject before participation.

Image postprocessing and analysis Prior to image analysis, the ECG-gated phases of each scan were averaged according to a previously published protocol to obtain time-averaged CT volumes with improved signal-to-noise ratio (SNR) compared to the reduced SNR of the individual phases[17]. The time-averaged CT volumes, representing mid cardiac cycle, were analyzed using 3-dimensional image analysis software (Aquarius Intuition version 4.4; TeraRecon, San Mateo, CA, USA) by 2 independent experienced observers blinded to each other's outcomes. Outer-to-outer diameter and area were assessed along the perirenal aortic neck in cross sectional planes perpendicular to the center lumen line (CLL) at predefined levels (Figure 1): (a) just below the superior mesenteric artery; (b) upper edge of the highest renal artery; (c) lower edge of the lowermost renal artery (baseline); (d) just before the initial origin of the aneurysm (a fixed distance from baseline); and (e) every $5 \mathrm{~mm}$ below baseline down to the initial origin of the aneurysm.

Although the Anaconda AAA stent-graft is an infrarenal fixating and sealing device, suprarenal measurements were performed to assess potential suprarenal remodeling not related to the presence of the stent-graft. At each level, ellipses were drawn on the outer wall to assess area and minimum and maximum diameters, that is, minor and major axes of the ellipses, respectively. The minimum and maximum diameters were used to compute the average diameter, hereafter referred to as diameter. The 
Table 1. Overview of device-related complications during follow-up.

\begin{tabular}{lccl}
\hline Event & Patient & Months after EVAR & Intervention \\
\hline Type II endoleak & $\# 9$ & 42 & Coil embolization \\
Migration & $\# 21$ & 1 & $\begin{array}{l}\text { None; 10-mm migration with } \\
\text { adequate AAA exclusion }\end{array}$ \\
Limb occlusion & $\# 2$ & 27 & Recanalization and endolining \\
& $\# 2$ & 47 & Thrombectomy \\
& $\# 3$ & 27 & Recanalization and endolining \\
\hline
\end{tabular}

Abbreviations: AAA, abdominal aortic aneurysm; EVAR, endovascular aneurysm repair.

measurements were subdivided into 3 aortic zones: the suprastent-ring zone, the stentring sealing zone, and the infrastent-ring zone, hereafter referred to as the suprastent, the stent, and the infrastent zones (Figure 1). The suprastent zone included the suprarenal measurements, the stent zone included the infrarenal measurements starting from baseline down to the level of the most caudal point of the dual rings at 2 years follow-up, and the infrastent zone included the measurements below the stent zone down to the level of the origin of the aneurysm in the predischarge CT scan. Additionally, aneurysm sac diameters and downstream displacement of the dual rings were assessed.

Statistical analysis Data are presented as mean \pm standard deviation (range) or as median [interquartile range Q1, Q3] for normally or non-normally distributed data, respectively. Measurements were compared between time points by use of a linear mixed model repeated measures analysis for normally distributed data and the Friedman test with post hoc Wilcoxon signed-rank tests for non-normally distributed data. The autoregressive covariance model was found to be most appropriate for the repeated measures data in the mixed models. Bonferroni corrections were was applied.

Correlations between changes in aortic size in the different aortic zones were tested using the 2-tailed Pearson correlation coefficient (PCC). Additionally, correlations were tested between the change in a zone and the change in aneurysm sac size and between the change in a zone and the oversizing percentage.

Interobserver variability in measuring diameter and area was analyzed with the repeatability coefficient ( $\mathrm{RC}$ ) and the intraclass correlation coefficient (ICC). RC was calculated as 1.96 times the standard deviation of the differences between repeated measurements, according to the method of Bland and Altman[19]. The ICC was tested with a 2-way mixed model by absolute agreement. Mean values of the observers were used for further analysis. The threshold of statistical significance was $\mathrm{p}<0.05$ $(\mathrm{p}<0.01$ for Wilcoxon signed-rank tests to correct for multiple comparisons [type I error]). All statistical analyses were performed with SPSS Statistics (version 24.0; IBM Corporation, Armonk, NY, USA). 

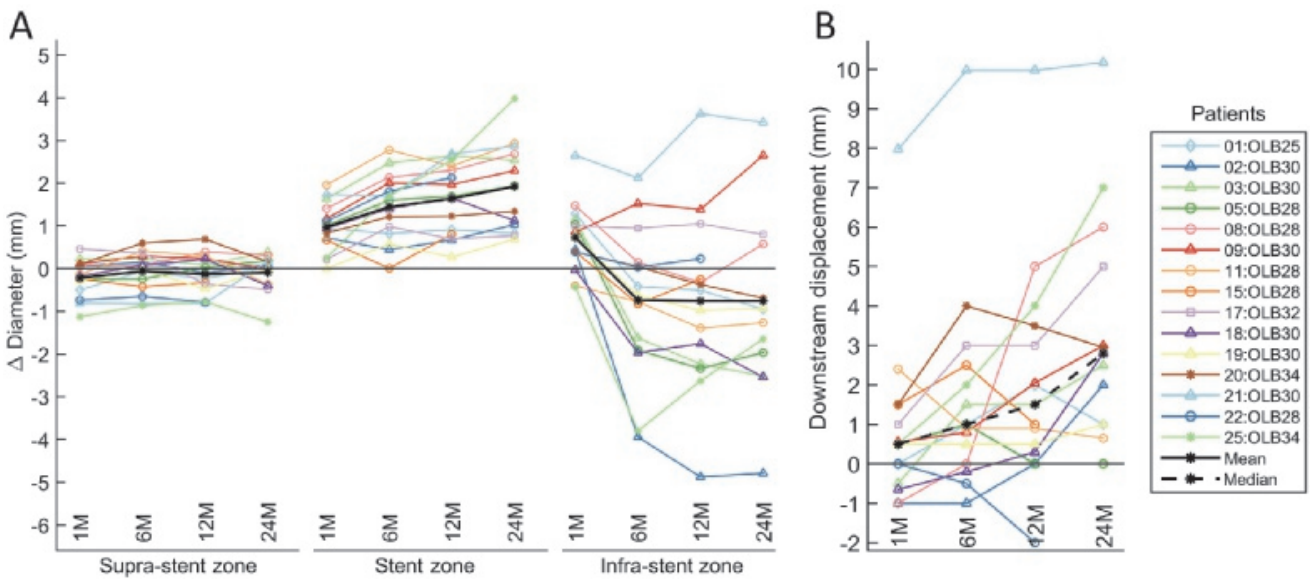

Figure 2. (A) Change in diameter of the 3 aortic zones and (B) downstream displacement of the dual rings during the 2-year follow-up period. M, months; OLB, main body device size.

\section{Results}

No type I or III endoleak was reported during a mean follow-up of $48.6 \pm 3.4$ months (range 44-55) for any of the patients in the study. One type II endoleak, 1 device migration, and 3 limb occlusions (2 patients) occurred (Table 1).

All measurements showed excellent agreement between observers (ICC 0.95 to $0.98, \mathrm{p}<0.001)$. The mean differences between repeated measurements of diameter, area, and device position, respectively, were $0.0 \pm 0.7 \mathrm{~mm}(0.0 \% \pm 2.4 \%), 0.9 \pm 31.6$ $\mathrm{mm}^{2}(0.3 \% \pm 4.6 \%)$, and $0.1 \pm 1.2 \mathrm{~mm}(0.7 \% \pm 6.0 \%)$, with an $\mathrm{RC}$ of $1.4 \mathrm{~mm}(4.7 \%)$, $61.9 \mathrm{~mm}^{2}(9.0 \%)$, and $2.4 \mathrm{~mm}(11.6 \%)$.

Table 2 presents the evolution of the diameter and area of the different aortic zones and the change with respect to the predischarge scan. Figure 2 provides a graphical representation of diameter changes and downstream displacement during follow-up. Two of the 15 patients did not complete the 2-year scan (1 voluntary withdrawal and 1 aneurysm-unrelated death). The average position of the most caudal point of the dual rings changed from $20.5 \pm 6.8 \mathrm{~mm}$ below baseline at discharge to $23.5 \pm 7.3 \mathrm{~mm}$ below baseline at 2 years, with a median change of $2.8 \mathrm{~mm}$ downstream $(\mathrm{p}=0.006$; Figure 2B).

Suprastent zone The median diameter and area of the suprastent zone at discharge were $25.5 \mathrm{~mm}$ and $511.9 \mathrm{~mm}^{2}$, respectively. The average change from discharge in diameter and area at 2 years was $-0.1 \pm 0.4 \mathrm{~mm}(-0.4 \% \pm 1.7 \%, \mathrm{p}=0.893)$ and $-2.9 \pm 17.2$ $\mathrm{mm}^{2}(-0.7 \pm 3.4 \%, \mathrm{p}=0.946)$, respectively. No significant changes were noted for any of the successive follow-up scans.

Stent zone The average diameter and area of the stent zone at discharge were $26.2 \pm 2.0 \mathrm{~mm}$ and $544.2 \pm 84.5 \mathrm{~mm}^{2}$, respectively. Diameter and area increased sig- 
A

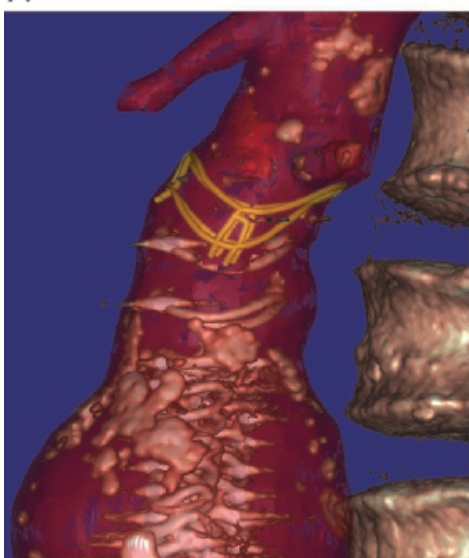

Discharge
B

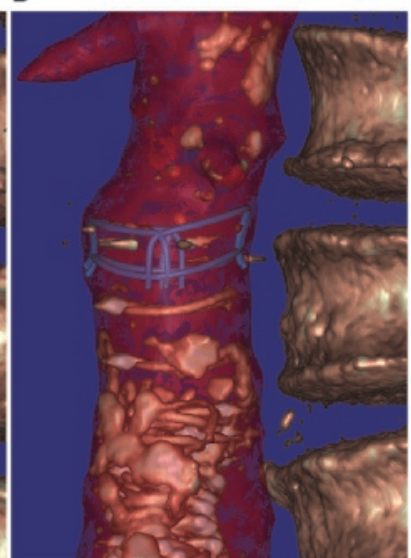

24 Months
C

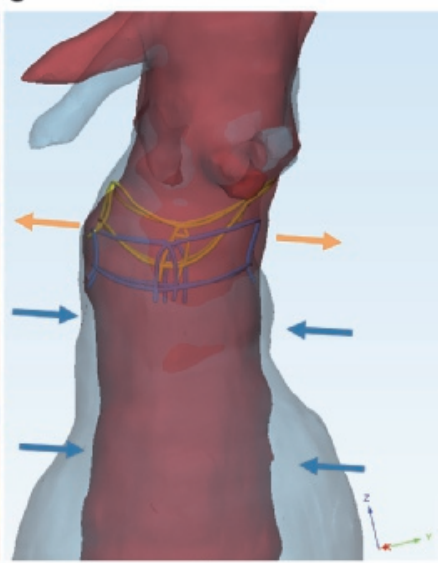

Combined

Figure 3. A clinical case (\#2) demonstrating a decrease in aortic neck size in the infrastent zone after endovascular aneurysm repair (EVAR). Computed tomography scan segmentations of the aorta (outer wall) and the proximal sealing rings (A) at discharge and (B) 2 years after EVAR were rigidly aligned based on vascular landmarks using Mimics/3-Matic software (Materialise, Leuven, Belgium) (C). Proximal ring-stent models were based on prior work[17]. Arrows indicate size increase (orange) or decrease (blue). The axes of the coordinate system $(\mathrm{C})$ denote the $\mathrm{x}$-(left-right), $\mathrm{y}$-(anteroposterior), and $\mathrm{z}$-(superior-inferior) directions.

nificantly between discharge and successive scans $(\mathrm{p}<0.001)$. The average change from discharge to 2 years' follow-up was $+1.9 \pm 1.0 \mathrm{~mm}(+7.3 \% \pm 4.0 \%, \mathrm{p}<0.001)$ and $+84.3 \pm 48.3 \mathrm{~mm}^{2}(+15.5 \% \pm 8.7 \%, \mathrm{p}<0.001)$, respectively. Between successive time points, the average percentage increase was greatest between discharge and 1 month by $+3.8 \% \pm 2.3 \%$ in diameter and $+7.8 \% \pm 4.8 \%$ in area $(\mathrm{p}<0.001)$. From 1 month, the average increase between successive time points was below $2 \%$ in diameter and below $4 \%$ in area. From 6 months, the increase between time points was not significant (diameter $\mathrm{p}>0.160$; area $\mathrm{p}>0.136$ ). Neck diameters in the stent zone did not exceed the main body stent-graft diameter.

Infrastent zone In the infrastent zone, mean diameter and area were $28.3 \pm 2.6 \mathrm{~mm}$ and $634.2 \pm 114.7 \mathrm{~mm}^{2}$ at discharge, respectively. At 1 month, mean diameter had significantly increased by $0.7 \pm 0.8 \mathrm{~mm}(2.7 \% \pm 2.9 \%, \mathrm{p}=0.038)$. From 1 to 6 months a significant decrease was observed in diameter and area by $1.5 \pm 1.4 \mathrm{~mm}(4.9 \% \pm 4.4 \%$, $\mathrm{p}<0.001)$ and $69.7 \pm 73.8 \mathrm{~mm}^{2}(9.7 \% \pm 8.9 \%, \mathrm{p}<0.001)$, respectively. The average change after 2 years was $-0.8 \pm 2.2 \mathrm{~mm}(-2.3 \% \pm 7.4 \%, \mathrm{p}>0.99)$ and $-34.6 \pm 102.3 \mathrm{~mm}^{2}$ $(-4.1 \% \pm 14.8 \%, \mathrm{p}>0.99)$, respectively. After 2 years, diameters had decreased in 9 patients and increased in 4 patients. A case example (\#2) presenting an evident decrease in size in the infrastent zone is shown in Figure 3.

An enlargement of $>2.5 \mathrm{~mm}$ in the infrastent zone diameter was found after 2 years in 2 patients (Figure 2): the patient with a type II endoleak 3.5 years after 
EVAR and the patient with a 10-mm caudal displacement of the dual rings 2 years after EVAR. This displacement had mainly occurred during the first month, after which the position stabilized. The type II endoleak was treated successfully by coil embolization. The device migration was treated conservatively. No device-related complications occurred. The aneurysm sac diameter remained stable. Due to the displacement, the infrastent zone comprised only the measurements at the initial origin of the aneurysm. In this patient, the device was oversized by $38 \%$ and was placed inclined in a $55^{\circ}$ infrarenally angulated aorta with a straight, 23-mm-long, 22 -mm-diameter neck that had no thrombus and $20 \%$ circumferential calcification. The infrastent neck diameters did not evolve beyond the main body device diameter.

In 2 other cases $(\# 8, \# 25)$, the size of the infrastent zone increased from 12 and 6 months, respectively, after an initial decrease in size, resulting in a minor $(<1 \mathrm{~mm})$ enlargement at last follow-up in 1 case (Figure 2A). This increase in size developed with a downstream displacement of the dual rings in both cases, resulting in 6- and 7mm caudal displacements, respectively, at 24 months (Figure 2B). Still, the aneurysm sac diameter had regressed by $\geq 20 \mathrm{~mm}$. The pertinent main bodies were oversized by $28 \%$ and $36 \%$, respectively. The infrarenal straight necks measured 26 and $41 \mathrm{~mm}$ in length by 21 and $25 \mathrm{~mm}$ in diameter and 0 and $100^{\circ}$ in infrarenal neck angulation, respectively, with no thrombus and minor calcification.

Correlations There was no correlation between changes in the stent zone and changes in the infrastent zone (1 year: PCC 0.18, $\mathrm{p}=0.513 ; 2$ years: PCC 0.23 , $\mathrm{p}=0.447)$. The change in diameter in the infrastent zone correlated positively with the change in aneurysm sac diameter at 1 year (PCC $0.69, \mathrm{p}=0.005$; Figure 4A) and 2 years (PCC 0.68, $\mathrm{p}=0.011$; Figure 4C). There was no correlation between the change in aneurysm sac diameter and the change in diameter in the stent zone at 1 year $(\mathrm{PCC}-0.01, \mathrm{p}=0.965$; Figure $4 \mathrm{~B})$ or 2 years $(\mathrm{PCC}-0.06, \mathrm{p}=0.848$; Figure 4D).

The oversizing percentages correlated positively with the percent change in diameter in the stent zone 1 year (PCC 0.54, $\mathrm{p}=0.037$ ) and 2 years ( $\mathrm{PCC} 0.61, \mathrm{p}=0.029$ ) after EVAR, but no correlation was found for the infrastent zone (1 year: PCC 0.32 , $\mathrm{p}=0.247 ; 2$ years: PCC 0.48, $\mathrm{p}=0.095)$.

\section{Discussion}

This study shows different remodeling of the perirenal aorta at the suprastent, stent, and infrastent zones after Anaconda implantation. While diameter and area increased in the stent zone of the infrarenal neck as a result of radial expansion of the nitinol sealing rings, a decrease was found in most patients in the infrastent neck zone during 2 years follow-up.

In line with our present observations in the stent zone, a recent retrospective study by Vukovic et al[18] reported a 2- to 4-mm increase in aortic diameter at the level of the upper and lower rings within 1 year after Anaconda implantation. This study suggested that the expansion of the Anaconda sealing rings leads to infrarenal neck expansion, stent-graft migration, and endoleak, even though diameters remained unchanged after 1 year. Additionally, the degree of ring flattening was below average in the patients with a type Ia endoleak, showing that in these cases the rings actually 
Table 2. Evolution of aortic diameter and area by aortic zone. ${ }^{a}$

\begin{tabular}{|c|c|c|c|c|c|}
\hline Aortic zone & $\begin{array}{l}\text { Discharge } \\
(\mathrm{n}=15)\end{array}$ & $\begin{array}{l}1 \text { Month } \\
(\mathrm{n}=15)\end{array}$ & $\begin{array}{l}6 \text { Months } \\
(\mathrm{n}=15)\end{array}$ & $\begin{array}{l}12 \text { Months } \\
(\mathrm{n}=15)\end{array}$ & $\begin{array}{l}24 \text { Months } \\
\quad(\mathrm{n}=13)\end{array}$ \\
\hline \multicolumn{6}{|l|}{ Suprastent } \\
\hline $\begin{array}{l}\text { Diameter, } \\
\mathrm{mm}\end{array}$ & $\begin{array}{c}25.5 \\
{[24.5,27.7]}\end{array}$ & $\begin{array}{c}25.0 \\
{[24.3,27.3]}\end{array}$ & $\begin{array}{c}25.5 \\
{[24.7,27.3]}\end{array}$ & $\begin{array}{c}25.3 \\
{[24.6,27.4]}\end{array}$ & $\begin{array}{c}25.6 \\
{[24.7,28.5]}\end{array}$ \\
\hline $\begin{array}{l}\triangle \text { Diameter, } \\
\mathrm{mm}\end{array}$ & & $\begin{array}{c}-0.2 \pm 0.4 \\
(-1.1 \text { to } 0.5) \\
p=0.073\end{array}$ & $\begin{array}{c}-0.1 \pm 0.5 \\
(-0.9 \text { to } 0.6) \\
p=0.890\end{array}$ & $\begin{array}{c}-0.1 \pm 0.5 \\
(-0.8 \text { to } 0.7) \\
p=0.303\end{array}$ & $\begin{array}{c}-0.1 \pm 0.4 \\
(-1.3 \text { to } 0.4) \\
p=0.893\end{array}$ \\
\hline$\underset{\%}{\triangle}$ Diameter, & - & $\begin{array}{c}-0.8 \pm 1.7 \\
(-4.4 \text { to } 1.9)\end{array}$ & $\begin{array}{c}-0.2 \pm 1.7 \\
(-3.4 \text { to } 1.9)\end{array}$ & $\begin{array}{c}-0.5 \pm 1.7 \\
(-3.2 \text { to } 2.2)\end{array}$ & $\begin{array}{c}-0.4 \pm 1.7 \\
(-4.9 \text { to } 1.4)\end{array}$ \\
\hline $\begin{array}{l}\text { Area, } \\
\mathrm{mm}^{2}\end{array}$ & $\begin{array}{c}511.9 \\
{[468.8,602.1]}\end{array}$ & $\begin{array}{c}492.2 \\
{[465.8,585.4]}\end{array}$ & $\begin{array}{c}509.9 \\
{[477.2,586.4]}\end{array}$ & $\begin{array}{c}502.1 \\
{[475.2,589.4]}\end{array}$ & $\begin{array}{c}515.7 \\
{[478.7,638.0]}\end{array}$ \\
\hline $\begin{array}{l}\triangle \text { Area } \\
\mathrm{mm}^{2}\end{array}$ & & $\begin{array}{c}-8.1 \pm 17.2 \\
(-41.3 \text { to } \\
16.5) \\
\mathrm{p}=0.107\end{array}$ & $\begin{array}{c}-1.5 \pm 18.9 \\
(-37.1 \text { to } 29.8) \\
\mathrm{p}=0.934\end{array}$ & $\begin{array}{c}-4.9 \pm 19.1 \\
(-35.3 \text { to } 33.9) \\
\mathrm{p}=0.389\end{array}$ & $\begin{array}{c}-2.9 \pm 17.2 \\
(-47.1 \text { to } 17.0) \\
\mathrm{p}=0.946\end{array}$ \\
\hline$\underset{\%}{\triangle}$ Area, & - & $\begin{array}{c}-1.6 \pm 3.2 \\
(-8.2 \text { to } 3.5)\end{array}$ & $\begin{array}{c}-0.4 \pm 3.4 \\
(-6.4 \text { to } 4.5)\end{array}$ & $\begin{array}{c}-1.0 \pm 3.4 \\
(-6.9 \text { to } 4.5)\end{array}$ & $\begin{array}{c}-0.7 \pm 3.4 \\
(-9.3 \text { to } 2.7)\end{array}$ \\
\hline \multicolumn{6}{|l|}{ Stent } \\
\hline $\begin{array}{l}\text { Diameter, } \\
\text { mm }\end{array}$ & $\begin{array}{c}26.2 \pm 2.0 \\
(22.9 \text { to } 30.2)\end{array}$ & $\begin{array}{c}27.2 \pm 1.9 \\
(24.0 \text { to } 31.0)\end{array}$ & $\begin{array}{c}27.7 \pm 2.2 \\
(24.5 \text { to } 31.4)\end{array}$ & $\begin{array}{c}27.9 \pm 2.2 \\
(25.1 \text { to } 31.7)\end{array}$ & $\begin{array}{c}28.1 \pm 2.2 \\
(25.1 \text { to } 33.1)\end{array}$ \\
\hline $\begin{array}{l}\triangle \text { Diameter, } \\
\mathrm{mm}\end{array}$ & & $\begin{array}{c}1.0 \pm 0.6 \\
(0.0 \text { to } 2.0) \\
\mathrm{p}<0.001\end{array}$ & $\begin{array}{c}1.4 \pm 0.8 \\
(0.0 \text { to } 2.8) \\
\mathrm{p}<0.001\end{array}$ & $\begin{array}{c}1.6 \pm 0.8 \\
(0.3 \text { to } 2.7) \\
\mathrm{p}<0.001\end{array}$ & $\begin{array}{c}1.9 \pm 1.0 \\
(0.7 \text { to } 4.0) \\
\mathrm{p}<0.001\end{array}$ \\
\hline$\underset{\%}{\triangle}$ Diameter, & - & $\begin{array}{c}3.8 \pm 2.3 \\
(-0.0 \text { to } 8.4)\end{array}$ & $\begin{array}{c}5.6 \pm 3.2 \\
(-0.0 \text { to } 11.9)\end{array}$ & $\begin{array}{c}6.3 \pm 3.2 \\
(1.1 \text { to } 10.3)\end{array}$ & $\begin{array}{c}7.3 \pm 4.0 \\
(2.6 \text { to } 14.0)\end{array}$ \\
\hline $\begin{array}{l}\text { Area, } \\
\mathrm{mm}^{2}\end{array}$ & $\begin{array}{c}544.2 \pm 84.5 \\
(412.8 \text { to } \\
717.2)\end{array}$ & $\begin{array}{c}584.4 \pm 83.8 \\
(453.6 \text { to } \\
756.1)\end{array}$ & $\begin{array}{c}605.7 \pm 94.0 \\
(472.0 \text { to } \\
773.4)\end{array}$ & $\begin{array}{c}614.3 \pm 98.2 \\
(491.5 \text { to } \\
788.0)\end{array}$ & $\begin{array}{c}626.7 \pm 95.3 \\
(492.9 \text { to } \\
859.5)\end{array}$ \\
\hline $\begin{array}{l}\triangle \text { Area } \\
\mathrm{mm}^{2}\end{array}$ & - & $\begin{array}{c}40.2 \pm 23.0 \\
(0.3 \text { to } 75.5) \\
\mathrm{p}<0.001\end{array}$ & $\begin{array}{c}61.5 \pm 32.7 \\
(1.6 \text { to } 110.4) \\
p<0.001\end{array}$ & $\begin{array}{c}70.1 \pm 36.3 \\
(11.8 \text { to } 122.1) \\
\mathrm{p}<0.001\end{array}$ & $\begin{array}{c}84.3 \pm 48.3 \\
(30.7 \text { to } 193.6) \\
\mathrm{p}<0.001\end{array}$ \\
\hline$\underset{\%}{\triangle}$ Area, & - & $\begin{array}{c}7.8 \pm 4.8 \\
(-0.0 \text { to } 17.7)\end{array}$ & $\begin{array}{c}11.7 \pm 6.7 \\
(0.1 \text { to } 25.4)\end{array}$ & $\begin{array}{c}13.3 \pm 6.9 \\
(2.3 \text { to } 22.2)\end{array}$ & $\begin{array}{c}15.5 \pm 8.7 \\
(5.8 \text { to } 30.6)\end{array}$ \\
\hline \multicolumn{6}{|l|}{ Infrastent } \\
\hline $\begin{array}{l}\text { Diameter, } \\
\mathrm{mm}\end{array}$ & $\begin{array}{c}28.3 \pm 2.6 \\
(22.6 \text { to } 33.3)\end{array}$ & $\begin{array}{c}29.0 \pm 2.5 \\
(23.0 \text { to } 33.8)\end{array}$ & $\begin{array}{c}27.6 \pm 2.1 \\
(22.6 \text { to } 30.5)\end{array}$ & $\begin{array}{c}27.6 \pm 2.2 \\
(22.8 \text { to } 30.4)\end{array}$ & $\begin{array}{c}27.7 \pm 2.4 \\
(25.0 \text { to } 31.7)\end{array}$ \\
\hline $\begin{array}{l}\triangle \text { Diameter, } \\
\mathrm{mm}\end{array}$ & 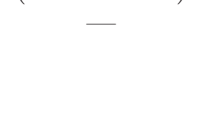 & $\begin{array}{c}0.7 \pm 0.8 \\
(-0.4 \text { to } 2.6) \\
\mathrm{p}=0.038\end{array}$ & $\begin{array}{c}-0.7 \pm 1.7 \\
(-3.9 \text { to } 2.1) \\
\mathrm{p}=0.319\end{array}$ & $\begin{array}{c}-0.8 \pm 2.0 \\
(-4.9 \text { to } 3.6) \\
\mathrm{p}=0.647\end{array}$ & $\begin{array}{c}-0.8 \pm 2.2 \\
(-4.8 \text { to } 3.4) \\
\mathrm{p}>0.99\end{array}$ \\
\hline$\underset{\%}{\triangle}$ Diameter, & - & $\begin{array}{c}2.7 \pm 2.9 \\
(-1.5 \text { to } 10.0)\end{array}$ & $\begin{array}{c}-2.3 \pm 5.6 \\
(-12.1 \text { to } 8.0)\end{array}$ & $\begin{array}{c}-2.4 \pm 6.7 \\
(-14.6 \text { to } 13.6)\end{array}$ & $\begin{array}{c}-2.3 \pm 7.4 \\
(-14.3 \text { to } 12.9)\end{array}$ \\
\hline $\begin{array}{l}\text { Area, } \\
\mathrm{mm}^{2}\end{array}$ & $\begin{array}{c}634.2 \pm 114.7 \\
(401.3 \text { to } \\
872.3)\end{array}$ & $\begin{array}{c}667.2 \pm 110.0 \\
(415.4 \text { to } \\
895.5)\end{array}$ & $\begin{array}{c}597.5 \pm 88.5 \\
(401.6 \text { to } \\
732.6)\end{array}$ & $\begin{array}{c}599.4 \pm 94.0 \\
(408.4 \text { to } \\
719.4)\end{array}$ & $\begin{array}{c}606.3 \pm 106.2 \\
(489.7 \text { to } \\
780.0)\end{array}$ \\
\hline $\begin{array}{l}\triangle \text { Area } \\
\mathrm{mm}^{2}\end{array}$ & - & $\begin{array}{c}33.0 \pm 34.0 \\
(-20.6 \text { to } \\
114.8) \\
\mathrm{p}=0.073\end{array}$ & $\begin{array}{c}-36.7 \pm 86.4 \\
(-232.4 \text { to } \\
93.4) \\
\mathrm{p}=0.283\end{array}$ & $\begin{array}{c}-34.8 \pm 92.2 \\
(-237.2 \text { to } \\
161.9) \\
\mathrm{p}=0.792\end{array}$ & $\begin{array}{c}-34.6 \pm 102.3 \\
(-232.9 \text { to } \\
151.6) \\
\mathrm{p}>0.99\end{array}$ \\
\hline$\underset{\%}{\triangle}$ Area, & - & $\begin{array}{c}5.6 \pm 5.9 \\
(-2.7 \text { to } 20.6)\end{array}$ & $\begin{array}{c}-4.6 \pm 11.6 \\
(-26.6 \text { to } 16.8)\end{array}$ & $\begin{array}{c}-4.2 \pm 13.3 \\
(-27.1 \text { to } 29.1)\end{array}$ & $\begin{array}{c}-4.1 \pm 14.8 \\
(-26.6 \text { to } 27.2)\end{array}$ \\
\hline
\end{tabular}

${ }^{a}$ Data are presented as the means \pm standard deviation (range) or as median [interquartile range Q1, Q3] as appropriate for the distribution of the data. 

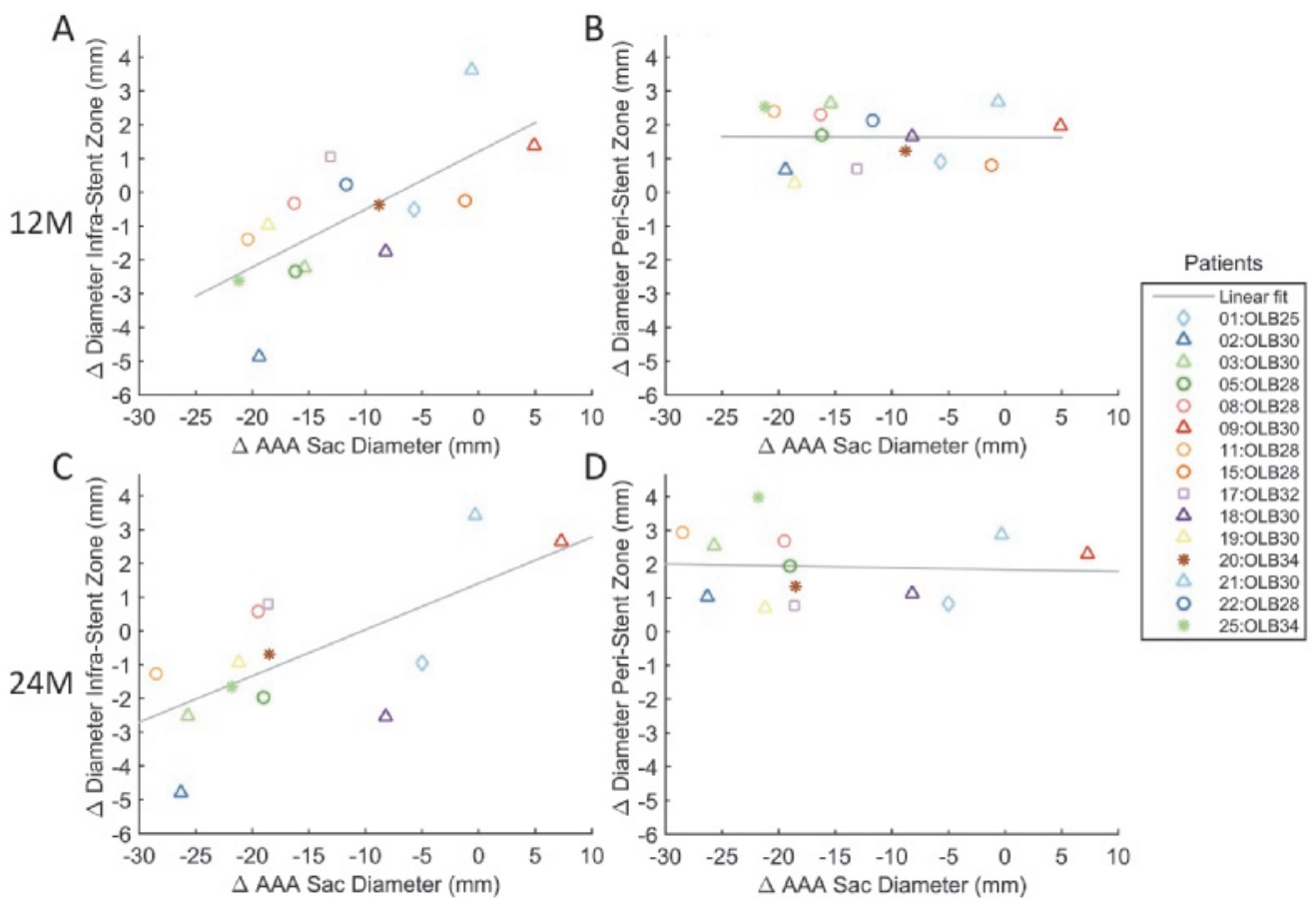

Figure 4. Correlation between change in aneurysm sac diameter and change in aortic diameter in the $(\mathrm{A}, \mathrm{C})$ infrastent zone and the $(\mathrm{B}, \mathrm{D})$ stent zone after $1(\mathrm{~A}, \mathrm{~B})$ and 2 years (C, D) follow-up. Negative values denote a decrease. The linear fit represents a least-squares fit for linear regression. AAA, abdominal aortic aneurysm; M, months; OLB, main body device size.

expanded less compared to most other patients. In our current study, the infrastent measurements showed that the proximal ring expansion had generally not affected the entire infrarenal neck segment despite a 31\% mean device oversize but had promoted diameter reduction of the neck below the nitinol sealing rings, which may in fact contribute to durable sealing and fixation. In contrast, 2 studies that investigated diameter changes at the distal end of the neck in various body-supported stent-grafts (Medtronic Talent, Cook Zenith, and Gore Excluder) did not evidence a reduction in size at the distal neck but rather growth, despite aneurysmal regression[20,21].

Kret et al[15] recently evaluated aortic neck remodeling at and $10 \mathrm{~mm}$ below the lowermost renal artery for 26 Cook Zenith, 26 Gore Excluder, 22 Medtronic Endurant, 10 Endologix Powerlink, and 2 TriVascular Ovation devices. Compared to the degree of neck dilation reported in this study at 2 years (mean change $3.8 \pm 2.4$ $\mathrm{mm}, 15.4 \% \pm 10.1 \%$ ), we observed an even lower degree of neck dilatation at similar levels (mean change $1.5 \pm 1.0 \mathrm{~mm}, 5.5 \% \pm 3.8 \%$ ) for the Anaconda device. Also, the clinical relevance of AND after EVAR is questionable since AND was not associated with adverse outcomes in multiple studies[13-16]. A study by Malas et al[22] on the performance of Lombard Medical's Aorfix stent-graft, an infrarenal fixating device that resembles the Anaconda but has an 8-mm proximal segment of concentric rings, 


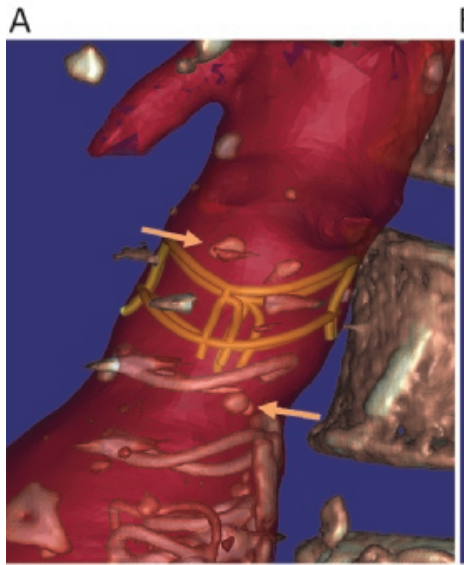

Discharge
B

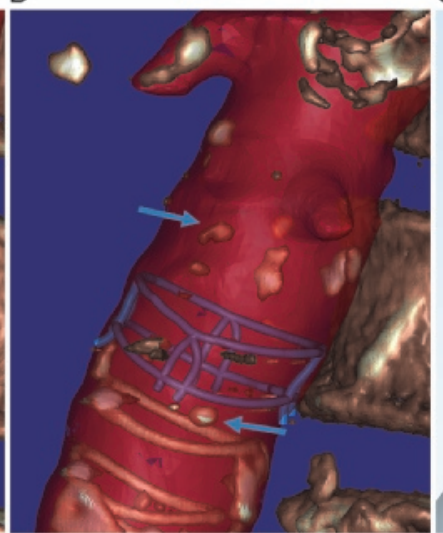

24 Months
C

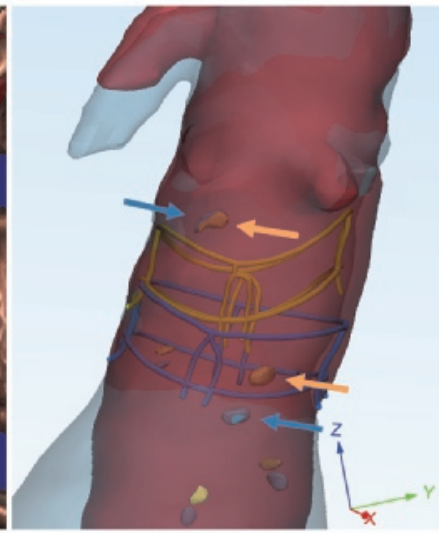

Combined

Figure 5. A case analysis $(\# 8)$ by calcification landmarks to evaluate potential axial remodeling. By aligning (C) the vasculature (A) at discharge and (B) 2 years after endovascular aneurysm repair (EVAR), it is clear that besides device migration, the perirenal aorta neck remodeled axially. The infrarenal neck length increased as a result of sac shrinkage. The arrows indicate calcification landmarks at discharge (orange) and 2 years after EVAR (blue). The axes of the coordinate system (C) denote the $\mathrm{x}$-(left-right), $\mathrm{y}$-(anteroposterior), and z-(superior-inferior) directions.

showed that for this device the aortic neck dilated at 7 and $15 \mathrm{~mm}$ below the lowermost renal artery but without an increased risk of migration.

Interestingly, a recent study on aortic neck evolution after implantation of TriVascular's Ovation stent-graft reported a slight decrease in diameters in the infrarenal segment above the polymer-filled sealing ring 2 years after EVAR[23]. Similar to the infrastent zone considered in the present study, this segment is free from outward radial force, which may have allowed aortic remodeling.

Remodeling of the distal neck may be related to remodeling mechanisms that also lead to aneurysm sac regression[24], notably, reversal of aortic wall inflammation[25], which involves tissue regeneration and shrinkage[26]. Additionally, the initial increase in infrastent neck size in the majority of patients after 1 month may be explained by the reconstructive phase of the inflammatory response, including granulation tissue formation, (myo)fibroblast proliferation, and collagen synthesis[26].

Even though the incidence of Anaconda main body migration appears to be low[2732], Vukovic et al[18] reported continuous migration of the main body during followup. However, their definition of migration overestimates downward body displacement due to the expansion and flattening of the proximal saddle-shaped rings over time. The average 6-mm increase in distance between the superior mesenteric artery and the Anaconda upper ring was reported as main body migration, while an average 3 -mm increase in renal artery to upper ring distance was found, which is similar to the displacement observed in our present study.

The downward movement of the peaks should not be considered migration, as it results from the adaptation of the sealing rings. We therefore use the lower valley of 
the dual ring to examine body migration distances. In our present study, we measured $>5$-mm downstream displacement of the main body in 3 cases that developed with an increase in infrastent neck diameter, though not resulting in any type I or III endoleak. Besides severe infrarenal neck angulation $\left(>90^{\circ}\right)$ in 1 of the 3 cases, there were no clear predisposing neck characteristics that may explain the downstream displacement. To determine whether true device migration or axial remodeling had occurred in these cases, we additionally assessed the device displacement in relation to calcification landmarks in the neck. In 2 of the 3 cases, the distance from baseline to calcification landmarks near the sealing rings had increased by 4 to $5 \mathrm{~mm}$, indicating that the neck remodeled axially under downward drag forces on the graft by the blood flow. In these cases, the true device migration actually constituted $<5 \mathrm{~mm}$. But more importantly, we observed an increase in neck length as a result of sac shrinkage. A case analysis is shown in Figure 5.

For the third case, we predominantly observed true migration, as elongation of the infrarenal neck was below $2 \mathrm{~mm}$ (Figure 6). Elongation of the aortic neck has also been observed by Litwinski et al[33], who discussed these migration phenomena. Notably, in these 3 patients the proximal sealing ring had directly lost most of its saddle shape at the predischarge scan, despite ample oversize $(>28 \%)$, indicating an immediate expansion of the stent-rings. In fact, in a previous study of the same cohort[17] these patients had the highest $(>94 \%)$ predischarge ring expansion percentages (diameter ring / nominal diameter ring x 100). Similarly, Schuurmann et al[34] found that the stent-graft had already expanded substantially at the first postoperative scan in their migration group specifically. Perhaps in these cases the aortic wall was not able to withstand the radial force of the stent-rings due to insufficient elastic recoil, resulting in ring expansion, neck dilatation, and migration or elongation. In our clinic, we currently avoid excessive oversize and advise an oversizing percentage of $10 \%$ to $20 \%$ for necks within the instructions for use and with less than $60^{\circ}$ infrarenal angulation. An in-depth individual assessment of the wall characteristics may be required in patient and device selection to identify such patients beforehand to allow for adequate treatment strategies.

Limitations The findings of the present study are limited by the small size of the study cohort and the lack of data regarding geometric changes beyond 2 years since patients were followed by duplex ultrasound examinations and plain radiography after that time. Nevertheless, reports from these non-CT examinations did not indicate any type I or III endoleak. Even though all changes from baseline in the stent zone were statistically significant, the size of the patient sample may have been too small to observe statistically significant changes in the other zones.

The strength of this investigation is that, to the best of our knowledge, no other study has reported in detail on size changes of the entire abdominal aortic neck segment. Thus, our data provide insight into neck remodeling after Anaconda implantation by differentiating between aortic zones. In addition, the repeatability of our measurements is similar to that reported by others $[35,36]$. Our study is unique in its approach because it longitudinally followed changes at standardized time points with a standardized thin-slice dynamic scan protocol that allowed measurements to be 


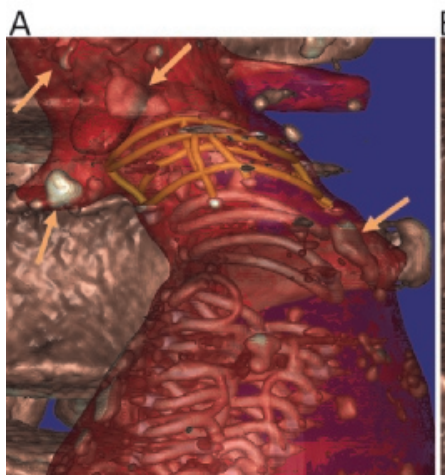

Discharge

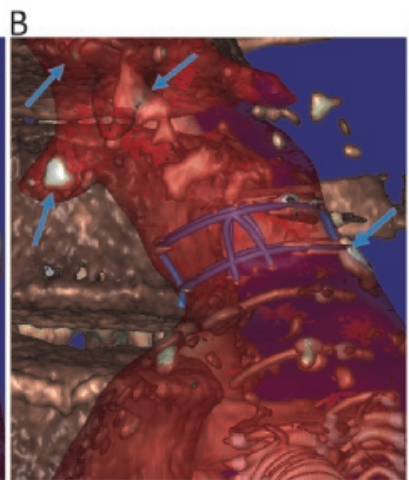

24 Months

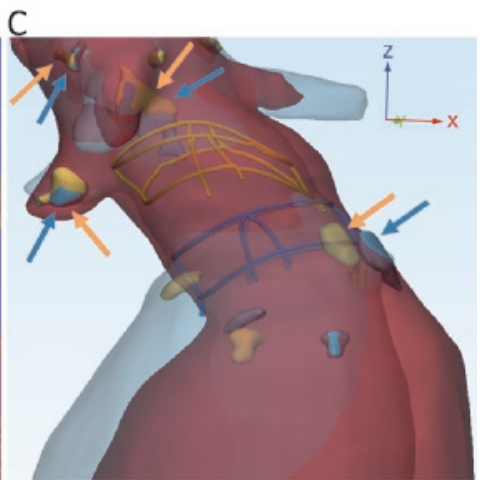

Combined

Figure 6. Another case analysis $(\# 21)$ by calcification landmarks demonstrated that the observed 10-mm main body displacement was predominantly caused by main body migration and not by axial remodeling of the aortic neck. Alignment (C) of the vasculature (A) at discharge and (B) 2 years after endovascular aneurysm repair (EVAR) shows that the position of a calcification pattern in the neck hardly changed. The arrows indicate calcification landmarks at discharge (orange) and 2 years after EVAR (blue). The axes of the coordinate system (C) denote the $\mathrm{x}$-(left-right), $\mathrm{y}$-(anteroposterior), and $\mathrm{z}$-(superior-inferior) directions.

repeated adequately at various aortic levels and at the same time during the cardiac cycle.

\section{Conclusion}

After EVAR using the Anaconda stent-graft, the infrarenal aortic neck accommodated to the expansion of the sealing rings at the stent sealing zone, but the neck below the stent zone decreased in size in the majority of patients. An evident increase in the infrastent zone was observed in 1 patient with 10-mm downstream displacement of the main body and in another patient who developed a type II endoleak. The suprarenal aortic diameter remained unchanged. Increasing diameter in the infrastent zone seems to relate to downstream device displacement, which may suggest that a decrease in size in the infrastent zone contributes to durable sealing and fixation of the Anaconda double stent-ring. Patients presenting such infrarenal neck remodeling below the stent sealing zone may require less regular follow-up, while patients presenting an increase may be prone to develop device migration and endoleak. A personalized follow-up scheme based on geometric neck remodeling after Anaconda implantation should be feasible if our observations are confirmed in larger, long-term studies.

\section{Acknowledgments}

The authors thank Prof. Dr. J.A.M. van der Palen (clinical epidemiologist, Medisch Spectrum Twente/University of Twente) for his assistance with the statistical analysis. 


\section{Declaration of conflicting interests}

R.H. Geelkerken is consultant for Terumo Aortic.

\section{Funding}

This study was funded in part by an unrestricted research grant from Vascutek Terumo, trading as Terumo Aortic, and in part by the PPP Allowance made available by Health Holland, Top Sector Life Sciences \& Health, to stimulate public-private partnerships.

\section{References}

1. Diehm N, Dick F, Katzen BT, Schmidli J, Kalka C, Baumgartner I. Aortic neck dilatation after endovascular abdominal aortic aneurysm repair: A word of caution. J Vasc Surg. 2008;47:886-92.

2. Kouvelos GN, Oikonomou K, Antoniou GA, Verhoeven ELG, Katsargyris A. A systematic review of proximal neck dilatation after endovascular repair for abdominal aortic aneurysm. J Endovasc Ther. 2017;24:59-67.

3. Filis KA, Galyfos G, Sigala F, Tsioufis K, Tsagos I, Karantzikos G, et al. Proximal aortic neck progression: Before and after abdominal aortic aneurysm treatment. Front Surg. 2017;4:1-6.

4. Oberhuber A, Buecken M, Hoffmann M, Orend K-HH, Mhling BM, Mühling BM. Comparison of aortic neck dilatation after open and endovascular repair of abdominal aortic aneurysm. J Vasc Surg. 2012;55:929-34.

5. Cao P, Verzini F, Parlani G, De Rango P, Parente B, Giordano G, et al. Predictive factors and clinical consequences of proximal aortic neck dilatation in 230 patients undergoing abdominal aorta aneurysm repair with self-expandable stent-grafts. J Vasc Surg. 2003;37:1200-5.

6. Dillavou ED, Muluk S, Makaroun MS. Is neck dilatation after endovascular aneurysm repair graft dependent? Results of 4 US Phase II trials. Vasc Endovascular Surg. 2005;39:47-54. 7. Sampaio SM, Panneton JM, Mozes G, Andrews JC, Noel AA, Kalra M, et al. Aortic Neck Dilation after Endovascular Abdominal Aortic Aneurysm Repair: Should Oversizing Be Blamed? Ann Vasc Surg. $2006 ; 20: 338-45$.

8. Dalainas I, Nano G, Bianchi P, Ramponi F, Casana R, Malacrida G, et al. Aortic neck dilatation and endograft migration are correlated with self-expanding endografts. J Endovasc Ther. 2007;14:318-23.

9. Napoli V, Sardella SG, Bargellini I, Petruzzi P, Cioni R, Vignali C, et al. Evaluation of the proximal aortic neck enlargement following endovascular repair of abdominal aortic aneurysm: 3-years experience. Eur Radiol. 2003;13:1962-71.

10. Hobo R, Buth J. Secondary interventions following endovascular abdominal aortic aneurysm repair using current endografts. A EUROSTAR report. J Vasc Surg. 2006;43:896-902.

11. De Bruin JL, Baas AF, Buth J, Prinssen M, Verhoeven ELG, Cuypers PWM, et al. Longterm outcome of open or endovascular repair of abdominal aortic aneurysm. N Engl J Med. 2010;362:1881-9.

12. Ilyas S, Shaida N, Thakor AS, Winterbottom A, Cousins C. Endovascular aneurysm repair (EVAR) follow-up imaging: the assessment and treatment of common postoperative complications. Clin Radiol. 2015;70:183-96.

13. Savlovskis J, Krievins D, De Vries JPPM, Holden A, Kisis K, Gedins M, et al. Aortic neck enlargement after endovascular aneurysm repair using balloon-expandable versus self-expanding endografts. J Vasc Surg. 2015;62:541-9.

14. Monahan TS, Chuter TAM, Reilly LM, Rapp JH, Hiramoto JS. Long-term follow-up of neck expansion after endovascular aortic aneurysm repair. J Vasc Surg. 2010;52:303-7.

15. Kret MR, Tran K, Lee JT. Change in Aortic Neck Diameter after Endovascular Aortic Aneurysm 
Repair. Ann Vasc Surg. 2017;43:115-20.

16. Tsilimparis N, Dayama A, Ricotta JJ. Remodeling of aortic aneurysm and aortic neck on followup after endovascular repair with suprarenal fixation. J Vasc Surg. 2015;61:28-34.

17. Koenrades MA, Klein A, Leferink AM, Slump CH, Geelkerken RH. Evolution of the Proximal Sealing Rings of the Anaconda Stent-Graft After Endovascular Aneurysm Repair. J Endovasc Ther. 2018;25:480-491.

18. Vukovic E, Czerny M, Beyersdorf F, Wolkewitz M, Berezowski M, Siepe M, et al. Abdominal aortic aneurysm neck remodeling after Anaconda stent graft implantation. J Vasc Surg. 2018;68:13541359.e2.

19. Martin Bland J, Altman D. Statistical methods for assessing agreement between two methods of clinical measurement. Lancet. 1986;327:307-10.

20. Kaladji A, Cardon A, Laviolle B, Heautot J-F, Pinel G, Lucas A. Evolution of the upper and lower landing site after endovascular aortic aneurysm repair. J Vasc Surg. 2012;55:24-32.

21. Singh-Ranger R, Adiseshiah M. Differing Morphological Changes following Endovascular AAA Repair Using Balloon-Expandable or Self-Expanding Endografts. J Endovasc Ther. 2000;7:479-85.

22. Malas MB, Jordan WD, Cooper MA, Qazi U, Beck AW, Belkin M, et al. Performance of the Aorfix endograft in severely angulated proximal necks in the PYTHAGORAS United States clinical trial. J Vasc Surg. 2015;62:1108-17.

23. De Donato G, Setacci F, Bresadola L, Castelli P, Chiesa R, Mangialardi N, et al. Aortic neck evolution after endovascular repair with TriVascular Ovation stent graft. J Vasc Surg. 2016;63:8-15.

24. Georgakarakos E, Georgiadis GS, Ioannou C V., Kapoulas KC, Trellopoulos G, Lazarides M. Aneurysm sac shrinkage after endovascular treatment of the aorta: Beyond sac pressure and endoleaks. Vasc Med (United Kingdom). 2012;17:168-73.

25. Kuivaniemi H, Ryer EJ, Elmore JR, Tromp G. Understanding the pathogenesis of abdominal aortic aneurysms. Expert Rev Cardiovasc Ther. 2015;13:975-87.

26. Rote NS, Huether SE, McCance KL. Innate Immunity: Inflammation. Pathophysiol - Biol Basis Dis Adults Child. 7th ed. [St. Louis, Missouri]: Elsevier Mosby; 2013. p. 195-220.

27. Freyrie A, Gallitto E, Gargiulo M, Faggioli G, Massoni CB, Mascoli C, et al. Results of the endovascular abdominal aortic aneurysm repair using the Anaconda aortic endograft. J Vasc Surg. 2014;60:1132-9.

28. Freyrie A, Gallitto E, Gargiulo M, Mascoli C, Faggioli G, Pini R, et al. Proximal aortic neck angle does not affect early and late EVAR outcomes: an Anaconda TM Italian registry analysis. J Cardiovasc Surg (Torino). 2014;55:671-7.

29. Rödel SGJ, Geelkerken RH, Prescott RJ, Florek HJ, Kasprzak P, Brunkwall J. The Anaconda AAA stent graft system: 2-year clinical and technical results of a multicentre clinical evaluation. Eur J Vasc Endovasc Surg. 2009;38:732-40.

30. Rödel SGJ, Zeebregts CJ, Huisman AB, Geelkerken RH. Results of the Anaconda endovascular graft in abdominal aortic aneurysm with a severe angulated infrarenal neck. J Vasc Surg. 2014;59:1495-1501.e1.

31. Saratzis N, Melas N, Saratzis A, Lazarides J, Ktenidis K, Tsakiliotis S, et al. Anaconda aortic stent-graft: single-center experience of a new commercially available device for abdominal aortic aneurysms. J Endovasc Ther. 2008;15:33-41.

32. Majumder B, Urquhart G, Edwards R, Irshad K, Velu R, Reid DB. Early clinical experience with the Anaconda re-deployable endograft in 106 patients with abdominal aortic aneurism: the west of Scotland Anaconda registry. Scott Med J. 2012;57:61-4.

33. Litwinski RA, Donayre CE, Chow SL, Song TK, Kopchok G, Walot I, et al. The role of aortic neck dilation and elongation in the etiology of stent graft migration after endovascular abdominal aortic aneurysm repair with a passive fixation device. J Vasc Surg. 2006;44:1176-81.

34. Schuurmann RCL, van Noort K, Overeem SP, van Veen R, Ouriel K, Jordan WD, et al. Determination of Endograft Apposition, Position, and Expansion in the Aortic Neck Predicts Type Ia Endoleak and Migration After Endovascular Aneurysm Repair. J Endovasc Ther. 2018;25:366-75.

35. Ghatwary TMH, Patterson BO, Karthikesalingam A, Hinchliffe RJ, Loftus IM, Morgan R, 
et al. A systematic review of protocols for the three-dimensional morphologic assessment of abdominal aortic aneurysms using computed tomographic angiography. Cardiovasc Intervent Radiol. $2013 ; 36: 14-24$.

36. Schuurmann RCL, Overeem SP, van Noort K, de Vries BA, Slump CH, de Vries J-PPM. Validation of a New Methodology to Determine 3-Dimensional Endograft Apposition, Position, and Expansion in the Aortic Neck After Endovascular Aneurysm Repair. J Endovasc Ther. 2018;25:358-65. 


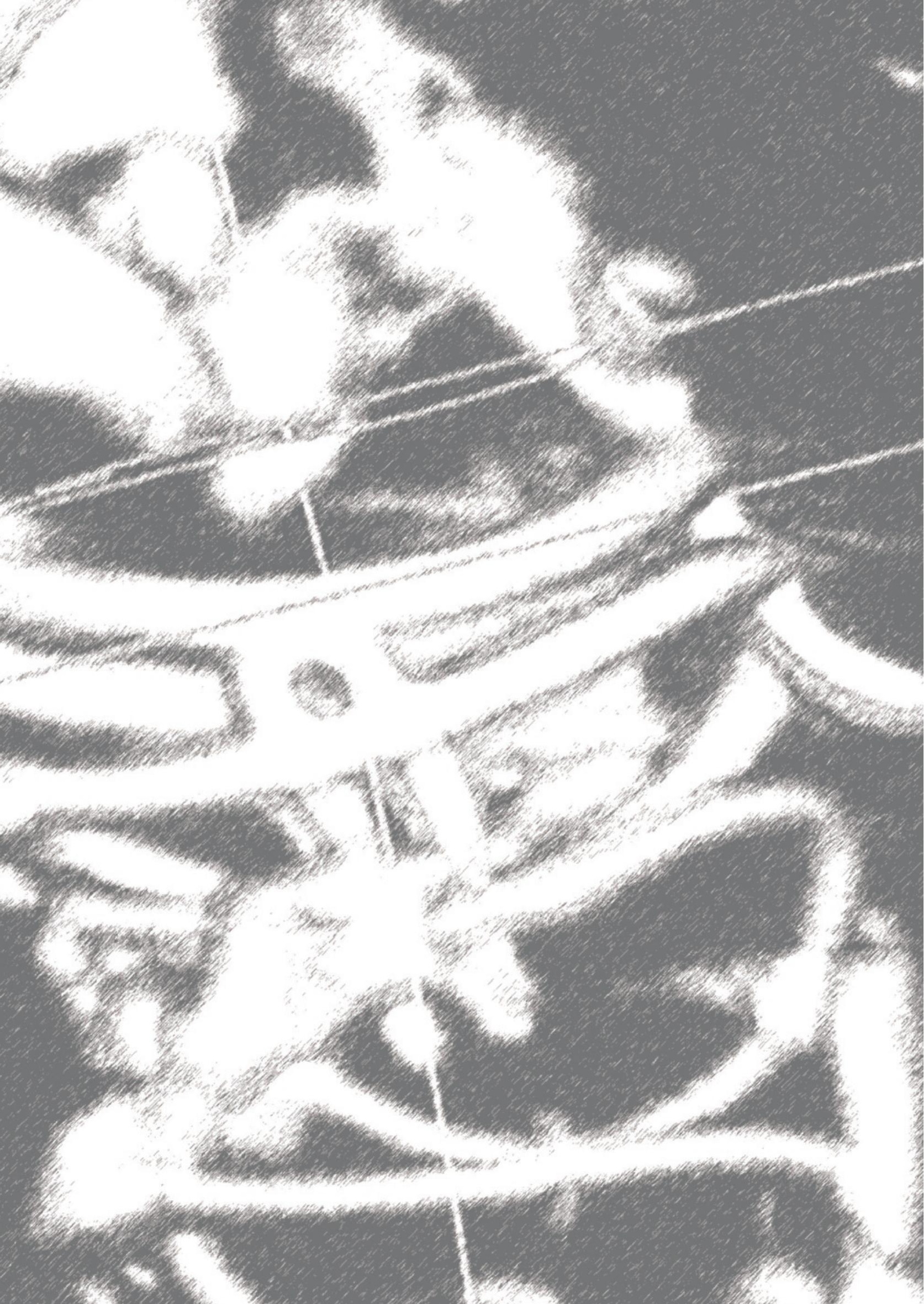





\section{Chapter 4}

\section{Peak and valley alignment of the Anaconda saddle-shaped sealing rings after endovascular aortic aneurysm repair: Implications for device positioning and sealing length}

Maaike A. Koenrades ${ }^{a, b, c *}$, Jaimy A. Simmering ${ }^{a, b}$, Cornelis H. Slump ${ }^{c}$, Robert H. Geelkerken ${ }^{a, b}$

${ }^{a}$ Multi-modality Medical Imaging (M3I) group, Faculty of Science and Technology, Technical Medical Centre, University of Twente, Enschede, the Netherlands

${ }^{b}$ Department of Vascular Surgery, Medisch Spectrum Twente, Enschede, the Netherlands

${ }^{c}$ Robotics and Mechatronics (RaM) group, Faculty of Electrical Engineering, Mathematics and Computer Science, Technical Medical Centre, University of Twente, Enschede, the Netherlands

Preliminary data from this study were presented at the 2018 Annual Meeting of the European Society for Vascular Surgery (September 25-28, 2018; Valencia, Spain). The presentation was awarded the $1^{\text {st }}$ prize for best poster presentation. 


\section{Abstract}

Objective: It was this study's aim to evaluate the extent of upstream and downstream displacement of the peaks and valleys of the saddle-shaped Anaconda double sealing ring following endovascular aneurysm repair (EVAR).

Methods: This study evaluated prospectively collected data of 15 patients who underwent elective EVAR with the Anaconda stent-graft between April 2014 and May 2015. Electrocardiogram-gated computed tomography examinations were conducted before discharge and after 1, 6, 12, and 24 months. Curvilinear distances along the aortic centerline were measured relative to the lowermost renal artery and tilt of the peaks and valleys toward the centerline was computed.

Results: The peaks had displaced significantly more than the valleys at all time points $(\mathrm{p}<0.001)$. At 1 month, 6 months, 12 months, and 24 months follow-up, the median displacement of the peaks and valleys was $-1.9 \mathrm{~mm}$ vs $0.3 \mathrm{~mm},-4.5 \mathrm{~mm}$ vs $-1.4 \mathrm{~mm},-5.9 \mathrm{~mm}$ vs $-1.2 \mathrm{~mm}$, and $-7.3 \mathrm{~mm}$ vs $-3.1 \mathrm{~mm}$, respectively. In 3 cases with substantial oversizing $(>30 \%)$, valleys had moved upstream at last follow-up, by at most $3 \mathrm{~mm}$. No renal artery coverage or renal complications were observed. The median tilt of the peaks and valleys toward the centerline decreased from $9.6^{\circ}$ at discharge to $6.3^{\circ}$ at 24 months $(\mathrm{p}=0.057)$ and from $8.7^{\circ}$ to $5.3^{\circ}(\mathrm{p}=0.006)$, respectively.

Conclusion: Alignment of the peaks and valleys in the proximal saddle shape during 24 months follow-up evolved mostly by downward displacement of the peaks and a decrease in tilt. The observations suggest that with moderate oversizing the flattening of the saddle shape does not pose a risk for inadvertent coverage of the renal artery ostia by the valleys, which propounds juxtarenal placement of the stent-ring valleys as a feasible technique that may be beneficial for stent-graft sealing apposition and positional stability in the long term.

Keywords: Endovascular aortic aneurysm repair, device positioning, Anaconda stent-graft, renal artery coverage, sealing length, infrarenal neck, geometry 


\section{Introduction}

In endovascular aneurysm repair (EVAR), appropriate positioning of a stent-graft in the infrarenal aortic neck is crucial to obtain an adequate seal with sufficient overlap between the stent-graft and the aortic wall. Loss of sealing length can lead to type Ia endoleak, one of the most frequent and troublesome complications after EVAR $[1,2]$. A stent-graft should be positioned as close as possible to the renal arteries to fully utilize the available neck length[3]. Positioning strategy may be particularly relevant for the Anaconda stent-graft (Terumo Aortic, Inchinnan, Scotland, UK). This stentgraft has a different conformability compared to most other stent-grafts due to its saddle-shaped double stent-ring design for proximal infrarenal fixation and sealing. Once deployed, the double stent-ring forms a saddle-shape-like-configuration with two peaks and two valleys at each stent-ring.

It is known from previous research that the saddle-shape flattens as the stentrings expand during the first 6 to 12 months after EVAR with the greatest degree of expansion during the first month[4]. With this change in geometry, the valleys may migrate upstream. As a result, the instructions for use (IFU) require placement of the proximal peaks below the level of the renal arteries[5]. However, the saddle-shape design allows the peaks to be placed pararenally with the valleys accommodating the lower edge of the renal ostia. While upstream movement of the valleys has been discussed and observed during the first few days after implantation[6-9], so far only one retrospective study has investigated changes in distance between the proximal stent-ring and the visceral arteries[10]. However, since baseline CTA scans were taken within 3 months after EVAR with a slice thickness up to $3 \mathrm{~mm}$, the data provided in this study does not seem sufficiently accurate to evaluate the extent of upstream valley displacement. Additionally, with the static CT acquisition, images may have been obtained anywhere during systole or diastole, which could have further affected the measurements. In the present study, we evaluated a series of electrocardiogramgated CT scans that were prospectively acquired according to a standardized study protocol starting directly before discharge. The objective of this study was to define in detail the evolution and extent of upstream and downstream displacement of the peaks and valleys of the proximal Anaconda saddle-shaped sealing ring following EVAR.

\section{Methods}

This is a retrospective evaluation of prospectively collected data of 15 asymptomatic abdominal aortic aneurysm (AAA) patients (mean age $72.8 \pm 3.7 ; 14$ men) who underwent elective EVAR with an Anaconda AAA stent-graft between April 2014 and May 2015. Patients were concurrently enrolled in a clinical trial (Trialregister.nl identifier NTR4276) and signed an informed consent form approved by the institutional review board. Patients were followed by noncontrast electrocardiogram (ECG)-gated computed tomography (CT) scans before discharge and after 1, 6, 12, and 24 months of follow-up. A complete description of the patient sample, including anatomic characteristics, the image acquisition protocol, and the ECG-gated CT scan processing prior to analysis are published elsewhere[4]. The ECG-gated CT scans were reconstructed into 10 consecutive phases with a slice thickness of $1 \mathrm{~mm}$ and a slice increment 

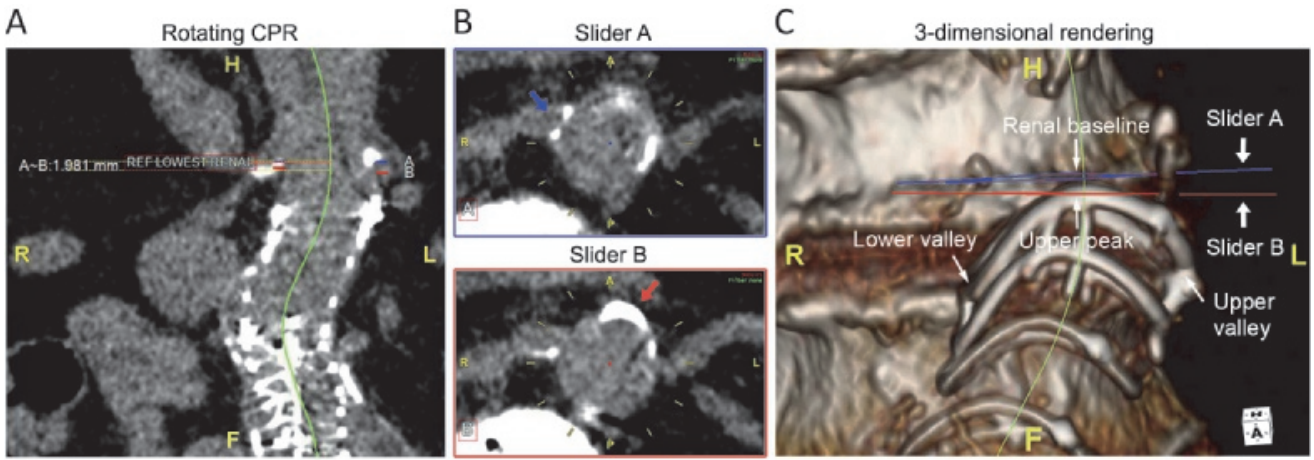

Figure 1. Computed tomography distance measurements on a TeraRecon workstation by (A) CPR imaging and (C) 3-dimensional volume rendering using (B) two cross-sectional planar reconstructions perpendicular to the centerline that can be shifted along the centerline by two orthogonal sliders. The A-slider (blue) was set to the lower edge of the lowermost renal artery (baseline; blue arrow). In this example, the B-slider (red) was set to the anterior peak of the proximal stent-ring (red arrow). The peak and valley that were positioned most proximally were defined as the upper peak and valley, respectively. The lower peak and valley refer to the other more distal peak and valley, respectively. $\mathrm{H}$, head; F, foot; $\mathrm{R}$, right; L, left; A, anterior; P, posterior.

(overlap) of $0.5 \mathrm{~mm}$. Two of the 15 patients did not complete the 24 -month scan (1 voluntary withdrawal and 1 non AAA-related death). Main body oversizing was based on inner wall diameters on preoperative static CTA scans and ranged from 17 to $47 \%$ (mean $31 \pm 9 \%$ ).

Image analysis Phase-averaged CT volumes with sufficient signal-to-noise ratio resulting from postprocessing were analyzed using 3-dimensional image analysis software (Aquarius Intuition version 4.4, TeraRecon, San Mateo, CA, USA). The phaseaveraged volumes represent mid cardiac cycle. An experienced observer performed the image analysis on all scans. Aortic centerlines were manually drawn using placement of points on standard planar reconstructions with subsequent automated interpolation generating sub-mm orthogonal planar reconstruction images. Centerlines were refined in curved planar reformation (CPR) images while rotating around the centerline. Curvilinear distances were measured along the aortic centerline, from the lower edge of the lowermost renal artery (baseline) to the two peaks and two valleys of the proximal stent-ring (Figure 1). The slope of the peaks and valleys toward the centerline was determined from the distance measurements by calculating the angle between the axis perpendicular to the centerline and the axis of the peaks/valleys (illustrated in Appendix A, Figure S1). This methodology resembles a previously validated approach to assess tilt[3].

Statistics Interobserver variability in measurement of distances and tilt was assessed with the intraclass correlation coefficient (ICC) and the repeatability coefficient (RC). Hereto, a second experienced observer independently performed all measure- 
D

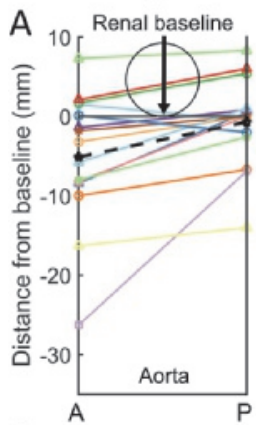

$1 \mathrm{M}$

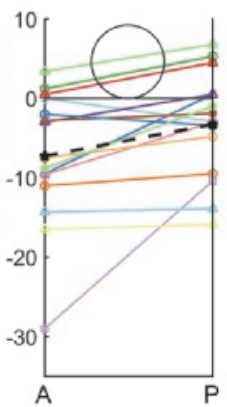

$6 \mathrm{M}$

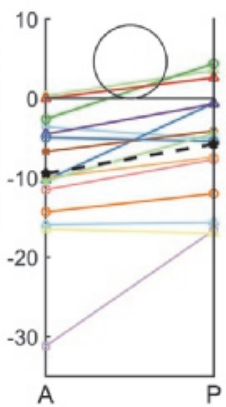

$12 \mathrm{M}$

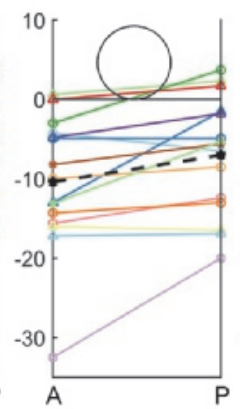

$24 \mathrm{M}$

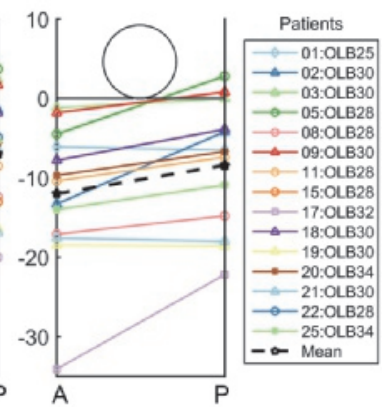

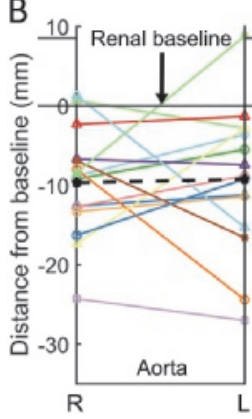
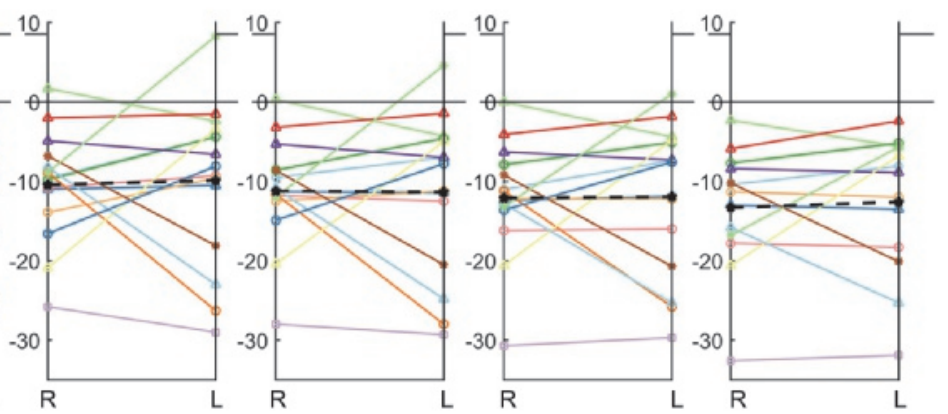

Figure 2. (A) and (B) are a schematic representation of proximal stent-ring peak and valley positions relative to renal artery baseline. (A) represents a lateral view; (B) a frontal anterior to posterior view. In two cases $(\# 19,25)$ with a $>90^{\circ}$ angulated infrarenal neck the body rotated by $90^{\circ}$ such that the valleys were in anteroposterior direction and the peaks in lateral direction[11]. In all other cases, the peaks were in anteroposterior direction and the valleys in lateral direction. Patient numbering refers to study identification numbering. A, anterior; P, posterior; R, right; L, left; D, discharge; M, months after EVAR; OLB, main body device size.

ments on the first and last postoperative scan of each patient, which was found to achieve sufficient power. The RC is defined as 1.96 times the standard deviation of the difference between two repeated measurements covering $95 \%$ of the expected variation[12]. The ICC was tested with a 2 -way mixed model by absolute agreement.

Descriptive statistics were performed including mean \pm standard deviation or median [ $1^{\text {st }}$ quartile, $3^{\text {rd }}$ quartile] as applicable for the type of distribution at hand. For parametric data, the change with time was analyzed by use of a linear mixed-model repeated measures analysis with a post-hoc Bonferroni correction to correct for multiple time point comparisons. For nonparametric data, the Friedman test was used instead with post hoc Wilcoxon signed-rank tests. Univariable analysis was performed for comparison of displacement between peak and valley subgroups using Wilcoxon signed-rank tests. Statistical significance was assumed when $\mathrm{p}<0.05$. Statistical analysis was performed with SPSS Statistics version 24.0 (IBM Corporation, Armonk, NY, USA). 
Table 1. Evolution of distances and tilt of the peaks and valleys of the proximal sealing ring. ${ }^{a}$

\begin{tabular}{|c|c|c|c|c|c|}
\hline & $\begin{array}{l}\text { Discharge } \\
\quad(\mathrm{n}=15)\end{array}$ & $\begin{array}{l}1 \text { Month } \\
(\mathrm{n}=15)\end{array}$ & $\begin{array}{l}6 \text { Months } \\
(\mathrm{n}=15)\end{array}$ & $\begin{array}{l}12 \text { Months } \\
\quad(\mathrm{n}=15)\end{array}$ & $\begin{array}{l}24 \text { Months } \\
\quad(\mathrm{n}=13)\end{array}$ \\
\hline \multicolumn{6}{|l|}{$\begin{array}{l}\text { Distance from } \\
\text { baseline }^{b}, \mathrm{~mm}\end{array}$} \\
\hline Upper peak & $\begin{array}{c}1.1 \pm 4.6 \\
(-6.9-8.8)\end{array}$ & $\begin{array}{c}-1.5 \pm 6.4 \\
(-13.9-8.3)\end{array}$ & $\begin{array}{c}-4.2 \pm 6.8 \\
(-16.6-4.6)\end{array}$ & $\begin{array}{l}-5.7 \pm 7.2 \\
(-20-3.7)\end{array}$ & $\begin{array}{c}-7.4 \pm 7.0 \\
(-22.2-2.8)\end{array}$ \\
\hline$\triangle$ Distance & - & $\begin{array}{c}-1.5 \\
{[-3.0,-0.5]} \\
p=0.158\end{array}$ & $\begin{array}{c}4.8 \\
{[-7.4,-2.2]} \\
p=0.002\end{array}$ & $\begin{array}{c}-5.7 \\
{[-8.5,-2.6]} \\
p=0.001\end{array}$ & $\begin{array}{c}-7.4 \\
{[-14.7,-4.7]} \\
\mathrm{p}<0.001\end{array}$ \\
\hline Lower peak & $\begin{array}{c}-5.5 \pm 8.3 \\
(-26.3-7.3)\end{array}$ & $\begin{array}{c}-7.8 \pm 8.7 \\
(-29.0-3.4)\end{array}$ & $\begin{array}{l}-10.0 \pm 8.3 \\
(-31.2-0.3)\end{array}$ & $\begin{array}{l}-10.9 \pm 8.7 \\
(-32.5-0.6)\end{array}$ & $\begin{array}{c}-12.3 \pm 8.6 \\
(-34.1--1.2)\end{array}$ \\
\hline$\triangle$ Distance & - & $\begin{array}{c}-1.6 \\
{[-3.5,-1.0]} \\
p=0.006\end{array}$ & $\begin{array}{c}-4.3 \\
{[-4.9,-3.1]} \\
\mathrm{p}<0.001\end{array}$ & $\begin{array}{c}-4.8 \\
{[-6.7,-3.4]} \\
p<0.001\end{array}$ & $\begin{array}{c}-7.2 \\
{[-8.5,-5.5]} \\
p<0.001\end{array}$ \\
\hline Upper valley & $\begin{array}{c}-7.4 \pm 6.5 \\
(-24.3-1.3)\end{array}$ & $\begin{array}{c}-7.8 \pm 6.6 \\
(-25.8-1.7)\end{array}$ & $\begin{array}{c}-9.4 \pm 6.9 \\
(-28.0-0.3)\end{array}$ & $\begin{array}{l}-10.3 \pm 7.4 \\
(-30.7-0.0)\end{array}$ & $\begin{array}{c}-12.0 \pm 7.6 \\
(-32.6--2.3)\end{array}$ \\
\hline$\triangle$ Distance & - & $\begin{array}{c}0.1 \\
{[-0.9,1.1]} \\
p>0.99\end{array}$ & $\begin{array}{c}-1.7 \\
{[-3.6,0.2]} \\
p=0.392\end{array}$ & $\begin{array}{c}-2.3 \\
{[-3.8,-0.5]} \\
p=0.150\end{array}$ & $\begin{array}{c}-3.3 \\
{[-8.3,-1.4]} \\
\mathrm{p}=0.024\end{array}$ \\
\hline Lower valley & $\begin{array}{c}-12.9 \pm 6.9 \\
(-27.0--2.3)\end{array}$ & $\begin{array}{l}-13.6 \pm 8.0 \\
(-29.0--2.0)\end{array}$ & $\begin{array}{c}-14.2 \pm 8.2 \\
(-29.3--3.2)\end{array}$ & $\begin{array}{c}-14.6 \pm 7.8 \\
(-29.7--4.1)\end{array}$ & $\begin{array}{c}-15.5 \pm 8.0 \\
(-31.9--5.6)\end{array}$ \\
\hline$\triangle$ Distance & - & $\begin{array}{c}-0.6 \\
{[-1.5,0.4]} \\
p>0.99\end{array}$ & $\begin{array}{c}-0.7 \\
{[-2.4,0.8]} \\
\mathrm{p}=0.967\end{array}$ & $\begin{array}{c}-1.6 \\
{[-3.5,0.3]} \\
\mathrm{p}=0.453\end{array}$ & $\begin{array}{c}-2.8 \\
{[-5.0,-1.0]} \\
\mathrm{p}=0.091\end{array}$ \\
\hline \multicolumn{6}{|l|}{$\operatorname{Tilt}^{c},{ }^{\circ}$} \\
\hline Peaks & $\begin{array}{c}9.6 \\
{[5.0,18.8]}\end{array}$ & $\begin{array}{c}7.2 \\
{[4.0,19.1]}\end{array}$ & $\begin{array}{c}6.2 \\
{[4.9,18.3]}\end{array}$ & $\begin{array}{c}5.5 \\
{[3.2,22.0]}\end{array}$ & $\begin{array}{c}6.3 \\
{[3.5,18.1]}\end{array}$ \\
\hline$\triangle$ Tilt & - & $\begin{array}{c}-1.4 \pm 5.0 \\
(-14.8-5.8) \\
\mathrm{p}=0.489\end{array}$ & $\begin{array}{c}-2.9 \pm 6.5 \\
(16.0-5.8) \\
\mathrm{p}=0.188\end{array}$ & $\begin{array}{c}-4.0 \pm 7.3 \\
(-20.3-5.0) \\
p=0.083\end{array}$ & $\begin{array}{c}-5.0 \pm 7.7 \\
(-21.8-6.0) \\
\mathrm{p}=0.057\end{array}$ \\
\hline Valleys & $\begin{array}{c}8.7 \\
{[4.8,18.2]}\end{array}$ & $\begin{array}{c}10.1 \\
{[3.3,20.8]}\end{array}$ & $\begin{array}{c}5.8 \\
{[2.5,16.7]}\end{array}$ & $\begin{array}{c}6.3 \\
{[1.1,14.2]}\end{array}$ & $\begin{array}{c}5.3 \\
{[1.0,6.8]}\end{array}$ \\
\hline$\triangle$ Tilt & {$[-$} & $\begin{array}{c}0.4 \pm 3.3 \\
(-5.4-5.3) \\
\mathrm{p}=0.561\end{array}$ & $\begin{array}{c}-1.7 \pm 3.2 \\
(-7.2-3.1) \\
p=0.073\end{array}$ & $\begin{array}{c}-2.5 \pm 3.6 \\
(-8.2-3.7) \\
\mathrm{p}=0.018\end{array}$ & $\begin{array}{c}-3.9 \pm 4.3 \\
(-13.3-4.9) \\
p=0.006\end{array}$ \\
\hline
\end{tabular}

${ }^{a}$ Data are presented as the mean \pm standard deviation (range) or as the median $\left[1^{\text {st }}\right.$ quartile, $3^{\text {rd }}$ quartile] as appropriate for the type of distribution. $\mathrm{P}$ values refer to discharge vs other time points.

${ }^{b}$ Negative and positive distances denote below and above renal artery baseline, respectively; Negative and positive change denote downstream and upstream displacement, respectively.

${ }^{c} \mathrm{~A}$ tilt of $0^{\circ}$ means perpendicular to the centerline. 


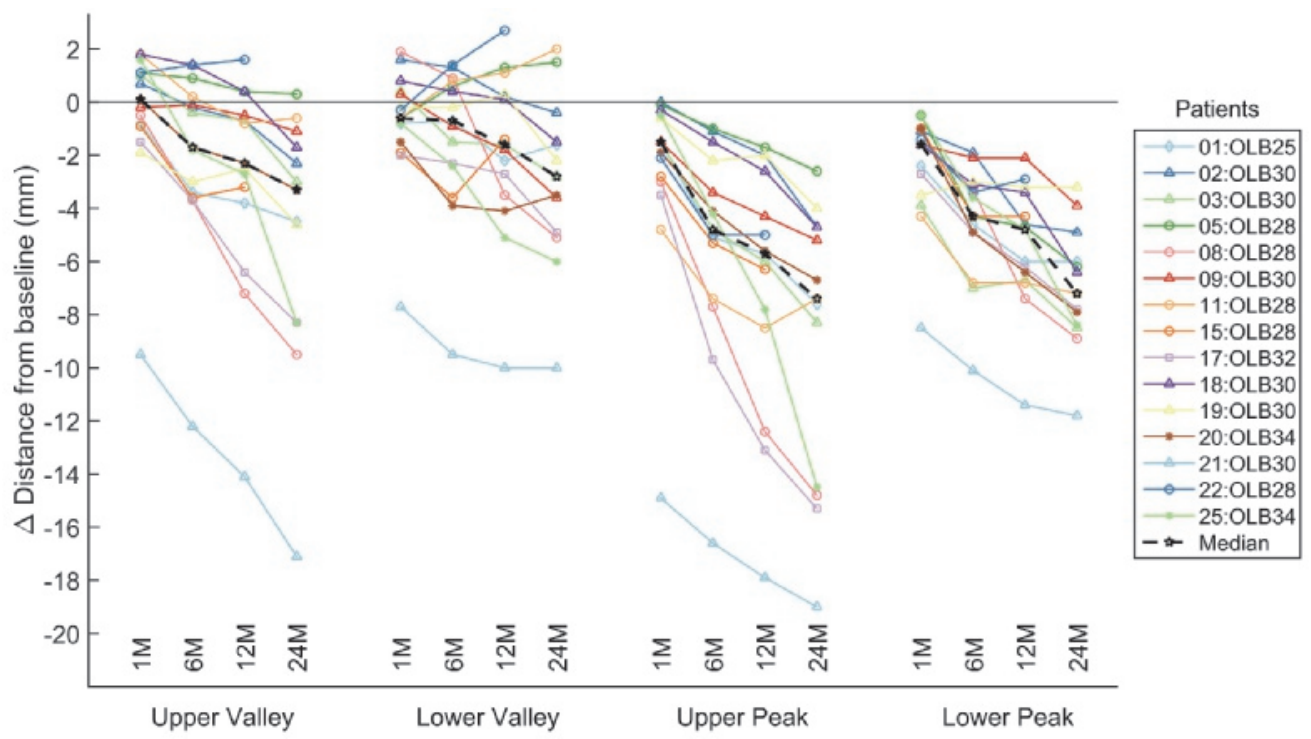

Figure 3. Displacement of the two peaks (upper and lower) and the two valleys (upper and lower) of the proximal stent-ring, showing the change in distance from the renal artery baseline through follow-up. Change is with respect to the discharge scan. Negative values represent downstream displacement; positive values represent upstream displacement. M, months after EVAR; OLB, main body device size.

\section{Results}

Distances between renal artery baseline and the peaks and valleys through follow-up are summarized in Table 1 and visualized for each patient in Figure 2. Upon discharge, peaks were positioned above renal artery baseline in 6 of the 15 patients. The change in distance from the predischarge scan is displayed in Figure 3 for each patient. The peaks displaced significantly more than the valleys at all time points $(\mathrm{p}<0.001)$. At 1 month, 6 months, 12 months, and 24 months follow-up, the median displacement of the peaks and valleys was $-1.9 \mathrm{~mm}[-2.7,-1.0]$ vs $0.3 \mathrm{~mm}[-1.1,0.7],-4.5 \mathrm{~mm}[-5.9,-2.7]$ vs $-1.4 \mathrm{~mm}[-2.8,0.6],-5.9 \mathrm{~mm}[-7.7,-3.2]$ vs $-1.2 \mathrm{~mm}[-3.9,0.2]$, and $-7.3 \mathrm{~mm}[-11.5$, $-4.7]$ vs $-3.1 \mathrm{~mm}[-6.9,-1.5]$, respectively, where negative values denote downstream displacement. Additionally, we found the upper valleys to move significantly more than the lower valleys between discharge and 24 months follow-up (-3.3 mm [-8.3, $-1.4]$ vs $-2.8 \mathrm{~mm}[-5.0,-1.0] ; \mathrm{p}=0.010)$. The displacement of the upper peaks did not significantly differ from the lower peaks $(-7.4 \mathrm{~mm}[-14.7,-4.7] \mathrm{vs}-7.2 \mathrm{~mm}[-8.5,-5.5]$; $\mathrm{p}=0.190)$. In three cases, one with the peaks positioned above renal artery baseline, one or both valleys had moved upstream by at most $3 \mathrm{~mm}$ at last follow-up (Figure 3 ). These three patients had the most pronounced saddle shapes upon discharge in this cohort with applied oversizing percentages $>30 \%$ and $<19^{\circ}$ tilt upon placement. At their last follow-up, the stent-rings had expanded to $>94 \%$ of the nominal flat ring diameter[4]. In seven other cases, an upstream displacement of the valleys of at most $2 \mathrm{~mm}$ occurred temporarily up to 6 or 12 months after EVAR, after which the valleys 
moved downstream (Figure 3). A typical case example is presented in Figure 4. The upstream movement of the valleys did not lead to renal artery coverage or any sign of renal complications.

Tilt of the proximal peaks and valleys is summarized in Table 1. In Figure 5, the tilt of the peaks and valleys during follow-up is displayed for each patient. At discharge, the median tilt of the peaks toward the aortic centerline was $9.6^{\circ}\left[5.0^{\circ}\right.$, $\left.18.8^{\circ}\right]$; the median tilt of the valleys was $8.7^{\circ}\left[4.8^{\circ}, 18.2^{\circ}\right]$. During follow-up, tilt decreased in cases with an initial higher tilt, indicating alignment toward the aortic axis (Figure 5). At 24 months follow-up, the median tilt of the peaks had decreased to $6.3^{\circ}\left(3.5^{\circ}, 18.1^{\circ} ; \mathrm{p}=0.057\right)$; the median tilt of the valleys had decreased to $5.3^{\circ}$ $\left(1.0^{\circ}, 6.8^{\circ} ; \mathrm{p}=0.006\right)$.

Interobserver agreement The repeated distance measurements for the peaks and valleys showed excellent agreement (peaks: ICC $>0.955, \mathrm{p}<0.001$; valleys: ICC $>0.970, \mathrm{p}<0.001)$. The mean difference between repeated measurements was -0.1 to $0.6 \mathrm{~mm}$ for the peaks and -0.1 to $0.5 \mathrm{~mm}$ for the valleys, with $95 \%$ of the variance within 2.7 and $3.5 \mathrm{~mm}$ and within 2.3 and $3.9 \mathrm{~mm}$, respectively. Excellent agreement was also observed for the assessment of tilt (peaks: ICC $>0.960, \mathrm{p}<0.001$; valleys: ICC $>0.956, \mathrm{p}<0.001)$. The mean interobserver difference for tilt was -0.8 to $0.4^{\circ}$ for the peaks and -1.3 to $-1.2^{\circ}$ for the valleys, with $95 \%$ of the variance within 4.7 to $7.1^{\circ}$ for the peaks and 3.9 to $5.8^{\circ}$ for the valleys.

\section{Discussion}

As the proximal sealing ring expanded over time, the valleys had moved upstream by at most $3 \mathrm{~mm}$ in 3 patients with substantial oversizing percentages $>30 \%$. No renal artery coverage or renal complications were observed. The unfolding and flattening of the peaks and valleys had resulted in a median $7 \mathrm{~mm}$ downstream displacement of the peaks and a median $3 \mathrm{~mm}$ downstream displacement of the valleys 2 years after EVAR. These results support the feasibility and the safety to place the valleys of the Anaconda stent-graft juxtarenally with moderate oversizing.

To prevent renal artery coverage by the valleys, the Anaconda IFU states placement of the proximal peaks below the level of the renal arteries[5]. Admittedly, the incidence of renal artery occlusion is rare. A recently published and to date largest single-centre study on the Anaconda stent-graft including 317 patients with a mean follow-up of 47 months did not report any renal artery occlusions[13]. Another singlecentre study including 177 Anaconda patients reported two (1.1\%) asymptomatic cases of late renal artery occlusion at 12 months post-EVAR[6]. In the present study, in 6 out of 15 patients one or both peaks of the Anaconda proximal stent-ring were placed above the lowest renal artery, c.q. outside the IFU, resulting in no coverage of renal artery ostia. Also Vukovic et al.[10] did not report any renal artery occlusions amongst 126 Anaconda patients at a median follow-up of 2.0 years. As opposed to upstream movement of the valleys, we primarily observe some downstream movement of both the peaks and valleys, similar to the study by Vukovic et al.[10]. While Vukovic[10] did not report on device positioning, we demonstrate that over time alignment of the peaks with the valleys may reduce the device length within 


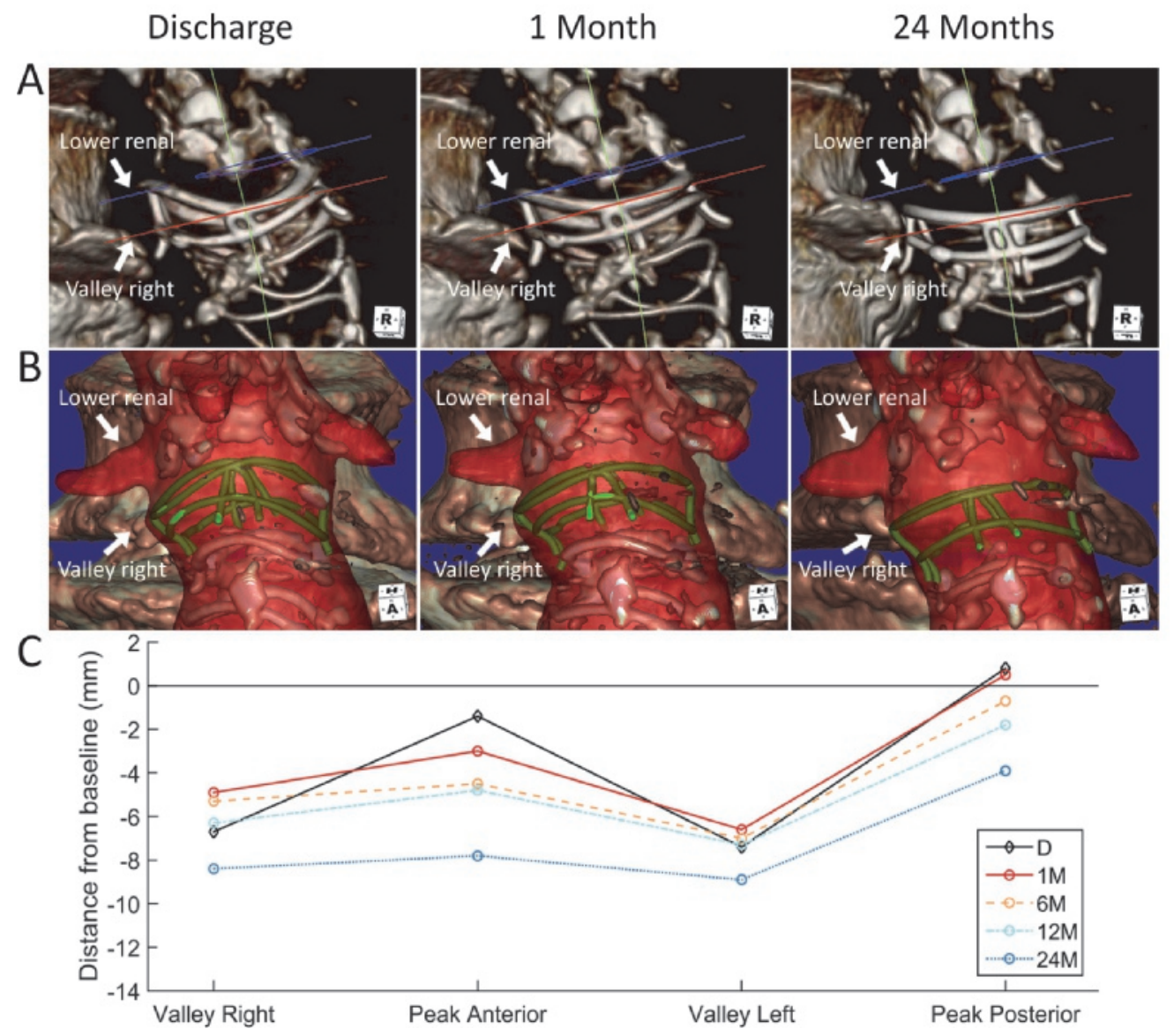

Figure 4. A typical case example (\#18) with temporary upstream movement of the valleys up to 6 months after EVAR due to flattening of the saddle-shape geometry. From 6 months, the valleys came back down. The main body (OLB30) was oversized by $22 \%$ with respect to inner wall diameter. The change in distance from the right (upper) valley to renal artery baseline is illustrated in 3-dimensional image sequences in (A) a right to left view showing the centerline and orthogonal sliders and (B) an anterior to posterior view with a segmentation of the vasculature. (C) Displays the distance to baseline for the two peaks and two valleys from discharge to 24 months follow-up. D, discharge; M, months after EVAR; H, head; F, foot; R, right; L, left; A, anterior; P, posterior. 

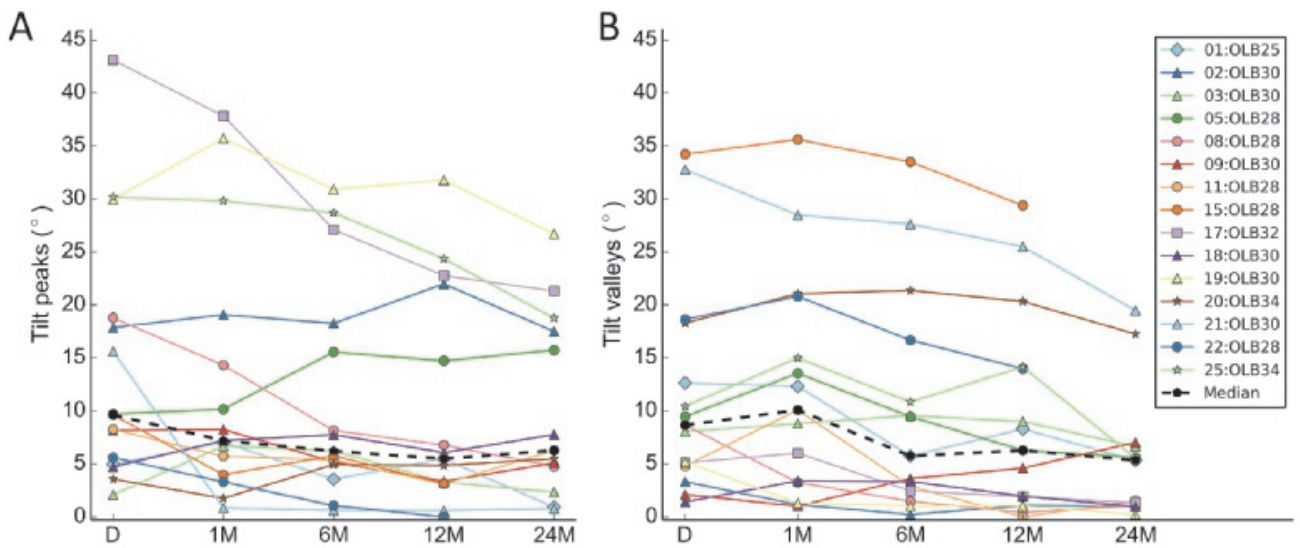

Figure 5. Evolution of tilt of the proximal stent-ring for (A) the peaks and (B) the valleys. A tilt of $0^{\circ}$ means perpendicular to the centerline. D, discharge; M, months after EVAR; OLB, main body device size.

the neck, leaving a proximal portion of the neck unused (Figure 2), which may be unfavorable for long-term sealing and fixation[14,15]. When the peaks are deployed directly below the level of the renal arteries while applying 10 to $20 \%$ oversizing, the valleys are then - depending on the body size - positioned approximately 7 to $13 \mathrm{~mm}$ below the renal arteries (Appendix A, Table S1). It should further be noted that the distance between the first and second stent-ring increases with main body size (Appendix A, Table S1). Deployment of the valleys more proximally to the renal arteries may contribute to durable sealing apposition and positional stability. A case example with evident pararenal peak and juxtarenal valley deployment with valleys as close as $3 \mathrm{~mm}$ to renal artery baseline is shown in Figure 6. Although in our patient sample higher device positioning did not lead to any adverse effect, one must be careful during device deployment and observant after implantation to recognize eventual coverage of the renal ostia as the consequences of renal occlusion can be severe[16]. Particularly patients with unfavorable neck characteristics may benefit from juxtarenal valley positioning in the long term. Others have treated necks as short as $3 \mathrm{~mm}$ by placing a stent in one or both renal arteries and pushing up the Anaconda main body by the repositioning system, with no medium term loss of renal patency or proximal seal[17]. However, we and others[18,19] do recommend to be cautions with EVAR outside the IFU, particularly with short necks $(<15 \mathrm{~mm})$ as both peaks and valleys seem to come down a few millimeters. In short necks, the alternative treatment option could be the use of suprarenal fixating devices in combination with fenestrations or chimneys, as they move the fixation zone into a potentially more stable but also more complicated aortic segment. Still, although the literature is conflicting on this topic[20], suprarenal fixation has been associated with higher rates of renal complications and longer length of hospital stay[21].

Furthermore, we observe a decrease in tilt for cases with an initial tilted orientation. This was also reflected by the downstream displacement of the upper peaks 
and/or valleys, which was observed to be as high as $15 \mathrm{~mm}$ with a $22^{\circ}$ decrease in tilt from $43^{\circ}$ to $21^{\circ}$ (\#17). This decrease in tilt should be considered in planning and follow-up as an initial higher device position in the neck may not be preserved. A slight reduction of tilt was also observed by Schuurmann et al. for other stentgrafts[15]. Downward drag forces of the blood flow on the flowsplitter concentrating on the most upstream fixating hooks is the most plausible hypothesis for this alignment with the aortic axis, especially since the drag forces appear to be greater in angulated anatomy[22], where a tilted device placement is more common. Additionally, this may have caused the hooks to further penetrate into the vessel wall, resulting in a few millimeters downstream displacement.

A limitation of this study may be the small patient number. However, considering the focus of this study to quantify in detail the evolution and extent of peak and valley displacement resulting from flattening of the stent-ring saddle shape, the employed data was appropriate for such in depth analysis. The thin-slice ECG-gated CT scans allowed for an accurate comparison of measurements taken at mid cardiac cycle. The low signal-to-noise ratio of the reconstructed individual phases did not allow for quantification of curvilinear distances during the cardiac cycle. However, qualitative evaluation of movement in dynamic cine-loop visualizations revealed practically no change in distance between the stent-ring peaks and the valleys during the cardiac cycle.

It must be noted that we measured distances along the centerline and not along the aortic contour, which may have moderately under- or overestimated the distances in case of highly curved anatomic segments[3]. However, there were no highly curved parts in the evaluated neck segments of the three cases in which the valleys had moved upstream. Furthermore, the interobserver agreements were excellent. The variance for the distance measurements was similar to the variance reported by England et al. for the assessment of migration distances[23] and slightly smaller compared to the variance reported for fabric distances along the aortic contour by Schuurmann et al. [3,24], which may be related to the smaller slice thickness of the data employed in the present study. Accordingly, variability of tilt estimations was also slightly lower in the present study compared to the study by Schuurmann et al.[24] Analysis of larger series is warranted to further evaluate the safety and efficacy of juxtarenal valley positioning.

\section{Conclusion}

Alignment of the peaks and valleys in the saddle shape of the Anaconda proximal sealing ring following EVAR evolved mostly by downward displacement of the peaks and a decrease of tilt toward the aortic centerline. We observe at most $3 \mathrm{~mm}$ upward valley movement after 24 months follow-up, exclusively in patients with substantial oversizing $>30 \%$, with no adverse effect. Since the degree of oversizing directly effects the extent of peak and valley displacement during the flattening of the saddle shape, it is unlikely that upward valley displacement would exceed the extent of upstream displacement observed in the present study when adhering to a 10-20\% oversizing. The observations propound juxtarenal placement of the stent-ring valleys as a feasible technique that may be beneficial for stent-graft sealing apposition and positional 


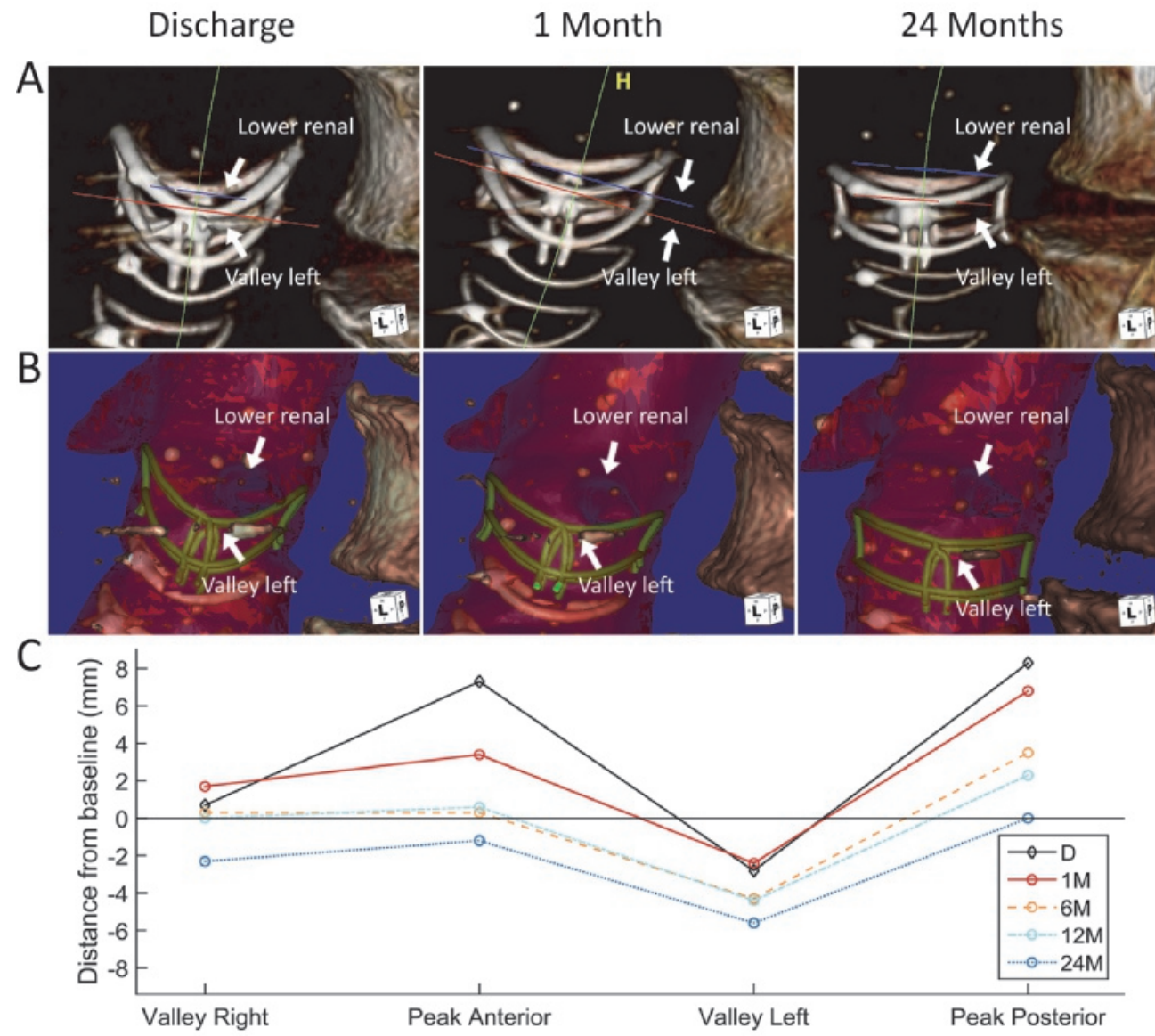

Figure 6. A case example (\#3) with placement outside the IFU with the peaks positioned pararenally and the valleys positioned juxtarenally as scallops around the renal artery ostia. The main body (OLB30) was oversized by $27 \%$ with respect to inner wall diameter. At discharge, the distance between renal artery baseline (left) and the ipsilateral valley was 3 $\mathrm{mm}$. The change in distance from this valley to baseline is illustrated in 3-dimensional image sequences in (A) a left to right view showing the centerline and orthogonal sliders and (B) with a segmentation of the vasculature. (C) Displays the distance to baseline for the two peaks and two valleys from discharge to 24 months follow-up. D, discharge; M, months after EVAR; H, head; F, foot; R, right; L, left; A, anterior; P, posterior. 
stability in the long term as the downward movement of the peaks reduces the length of stent-graft device within the neck. The safety and efficacy of juxtarenal valley positioning should be further investigated in a clinical registry.

\section{Acknowledgments}

The authors thank prof.dr. J.A.M. van der Palen (clinical epidemiologist, Medisch Spectrum Twente/University of Twente) for performing power calculations on observer variability assessment. The authors further thank I. Wolsink, MSc., and M.L. Groot Koerkamp, MSc., for examining the suitability of measurement methods during their Technical Medicine internship.

\section{Declaration of conflicting interests}

R.H. Geelkerken is consultant for Terumo Aortic.

\section{Funding}

This study was funded in part by an unrestricted research grant from Vascutek Terumo, trading as Terumo Aortic, and in part by the PPP Allowance made available by Health Holland, Top Sector Life Sciences \& Health, to stimulate public-private partnerships.

\section{References}

1. Thomas B, Sanchez L. Proximal migration and endoleak: Impact of endograft design and deployment techniques. Semin Vasc Surg. 2009;22:201-6.

2. Katzen BT, MacLean AA. Complications of endovascular repair of abdominal aortic aneurysms: A review. Cardiovasc Intervent Radiol. 2006;29:935-46.

3. Schuurmann RCL, Overeem SP, Ouriel K, Slump CH, Jordan WD, Muhs BE, et al. A Semiautomated Method for Measuring the 3-Dimensional Fabric to Renal Artery Distances to Determine Endograft Position after Endovascular Aneurysm Repair. J Endovasc Ther. 2017;24:698-706.

4. Koenrades MA, Klein A, Leferink AM, Slump CH, Geelkerken RH. Evolution of the Proximal Sealing Rings of the Anaconda Stent-Graft After Endovascular Aneurysm Repair. J Endovasc Ther. 2018;25:480-491.

5. Terumo Aortic. Anaconda ${ }^{\mathrm{TM}}$ AAA Stent Graft System: Combined IFU. Available from: http://www.vascutek.com/site/assets/files/3457/301-179_combined_anaconda_one-lok_ifu.pdf. Accessed 29 March 2019.

6. Freyrie A, Gallitto E, Gargiulo M, Faggioli G, Massoni CB, Mascoli C, et al. Results of the endovascular abdominal aortic aneurysm repair using the Anaconda aortic endograft. J Vasc Surg. 2014;60:1132-9.

7. Rödel SGJ, Geelkerken RH, Prescott RJ, Florek HJ, Kasprzak P, Brunkwall J. The Anaconda AAA stent graft system: 2-year clinical and technical results of a multicentre clinical evaluation. Eur J Vasc Endovasc Surg. 2009;38:732-40.

8. Karkos CD, Kapetanios DM, Anastasiadis PT, Grigoropoulou FS, Kalogirou TE, Giagtzidis IT, et al. Endovascular Repair of Abdominal Aortic Aneurysms with the Anaconda ${ }^{\mathrm{TM}}$ Stent Graft: Mid-term Results from a Single Center. Cardiovasc Intervent Radiol. 2015;38:1416-24.

9. Stella A, Freyrie A, Gargiulo M, Faggioli GL. The advantages of Anaconda endograft for AAA. J Cardiovasc Surg (Torino). 2009;50:145-52. 
10. Vukovic E, Czerny M, Beyersdorf F, Wolkewitz M, Berezowski M, Siepe M, et al. Abdominal aortic aneurysm neck remodeling after Anaconda stent graft implantation. J Vasc Surg. 2018;68:13541359.e2.

11. Rödel SGJ, Zeebregts CJ, Huisman AB, Geelkerken RH. Results of the Anaconda endovascular graft in abdominal aortic aneurysm with a severe angulated infrarenal neck. J Vasc Surg. 2014;59:1495-1501.e1.

12. Martin Bland J, Altman D. Statistical methods for assessing agreement between two methods of clinical measurement. Lancet. 1986;327:307-10.

13. Rödel SGJ, Zeebregts CJ, Meerwaldt R, van der Palen J, Geelkerken RH. Incidence and treatment of limb occlusion of the Anaconda endograft after endovascular aneurysm repair. J Endovasc Ther. 2018;26:113-20.

14. Zarins CK, Bloch DA, Crabtree T, Matsumoto AH, White RA, Fogarty TJ, et al. Stent graft migration after endovascular aneurysm repair: Importance of proximal fixation. J Vasc Surg. 2003;38:1264-72.

15. Schuurmann RCL, van Noort K, Overeem SP, van Veen R, Ouriel K, Jordan WD, et al. Determination of Endograft Apposition, Position, and Expansion in the Aortic Neck Predicts Type Ia Endoleak and Migration After Endovascular Aneurysm Repair. J Endovasc Ther. 2018;25:366-75.

16. Katzen BT, MacLean AA, Katzman HE. Retrograde migration of an abdominal aortic aneurysm endograft leading to postoperative renal failure. J Vasc Surg. 2005;42:784-7.

17. Bungay $\mathrm{P}$. The use of the Anaconda ${ }^{\mathrm{TM}}$ stent graft for abdominal aortic aneurysms. J Cardiovasc Surg (Torino). 2012;53:571-7.

18. Charbonneau P, Hongku K, Herman CR, Habib M, Girsowicz E, Doonan RJ, et al. Long-term survival after endovascular and open repair in patients with anatomy outside instructions for use criteria for endovascular aneurysm repair. J Vasc Surg. 2019; in press.

19. Herman CR, Charbonneau P, Hongku K, Dubois L, Hossain S, Lee K, et al. Any nonadherence to instructions for use predicts graft-related adverse events in patients undergoing elective endovascular aneurysm repair. J Vasc Surg. 2018;67:126-33.

20. Miller LE, Razavi MK, Lal BK. Suprarenal versus infrarenal stent graft fixation on renal complications after endovascular aneurysm repair. J Vasc Surg. 2015;61:1340-9.

21. Zettervall SL, Deery SE, Soden PA, Shean K, Siracuse JJ, Alef M, et al. Editor's Choice - Renal complications after EVAR with suprarenal versus infrarenal fixation among all users and routine users. Eur J Vasc Endovasc Surg. 2017;54:287-93.

22. Morris L, Delassus P, Walsh M, McGloughlin T. A mathematical model to predict the in vivo pulsatile drag forces acting on bifurcated stent grafts used in endovascular treatment of abdominal aortic aneurysms (AAA). J Biomech. 2004;37:1087-95.

23. England A, Garca-Fiana M, How T V., Vallabhaneni SR, McWilliams RG. The accuracy of computed tomography central luminal line measurements in quantifying stent graft migration. J Vasc Surg. 2012;55:895-905.

24. Schuurmann RCL, Overeem SP, van Noort K, de Vries BA, Slump CH, de Vries J-PPM. Validation of a New Methodology to Determine 3-Dimensional Endograft Apposition, Position, and Expansion in the Aortic Neck After Endovascular Aneurysm Repair. J Endovasc Ther. 2018;25:358-65. 


\section{Appendix A}

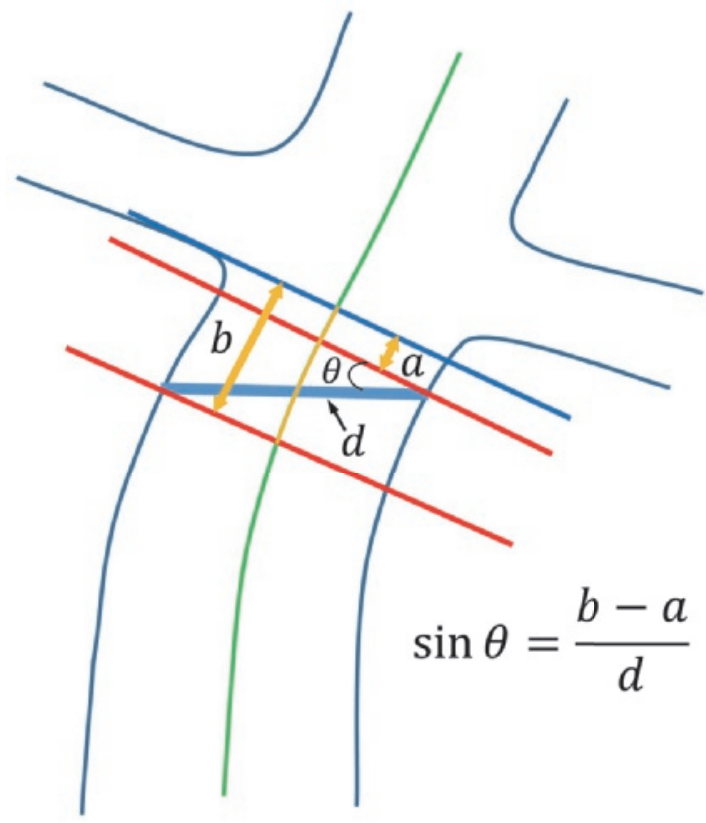

Figure S1. Illustration of the assessment of tilt for the peaks and valleys by calculating the inverse sine function for the angle between the axis of the proximal stent-ring peaks or valleys and the axis perpendicular to the centerline based on distance measurements, where $\mathrm{a}$ and $\mathrm{b}$ denote the curvilinear centerline distance from the lower edge of the lowermost renal artery to the upper and lower peak or the upper and lower valley, respectively, and d denotes the straight-line 3-dimensional distance from the upper to the lower peak or the upper to the lower valley. A tilt of 0 degrees denotes perpendicular to the centerline. 
Table S1. Ring profile device specifications of the Anaconda stent-graft double sealing ring. The distance between the peaks and valleys increases with oversize. Adapted from the Anaconda ring profile planning sheet with permission from the manufacturer.

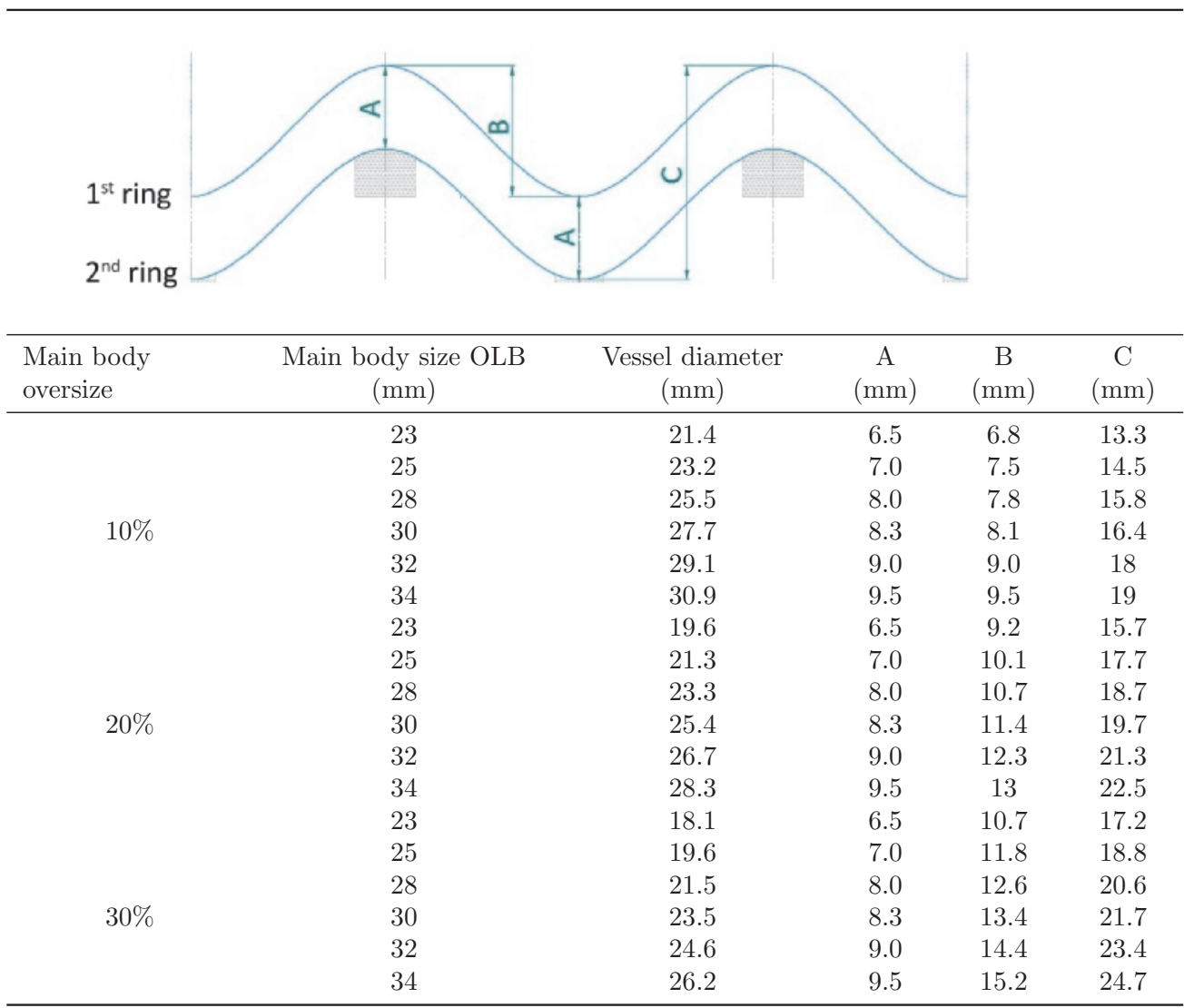




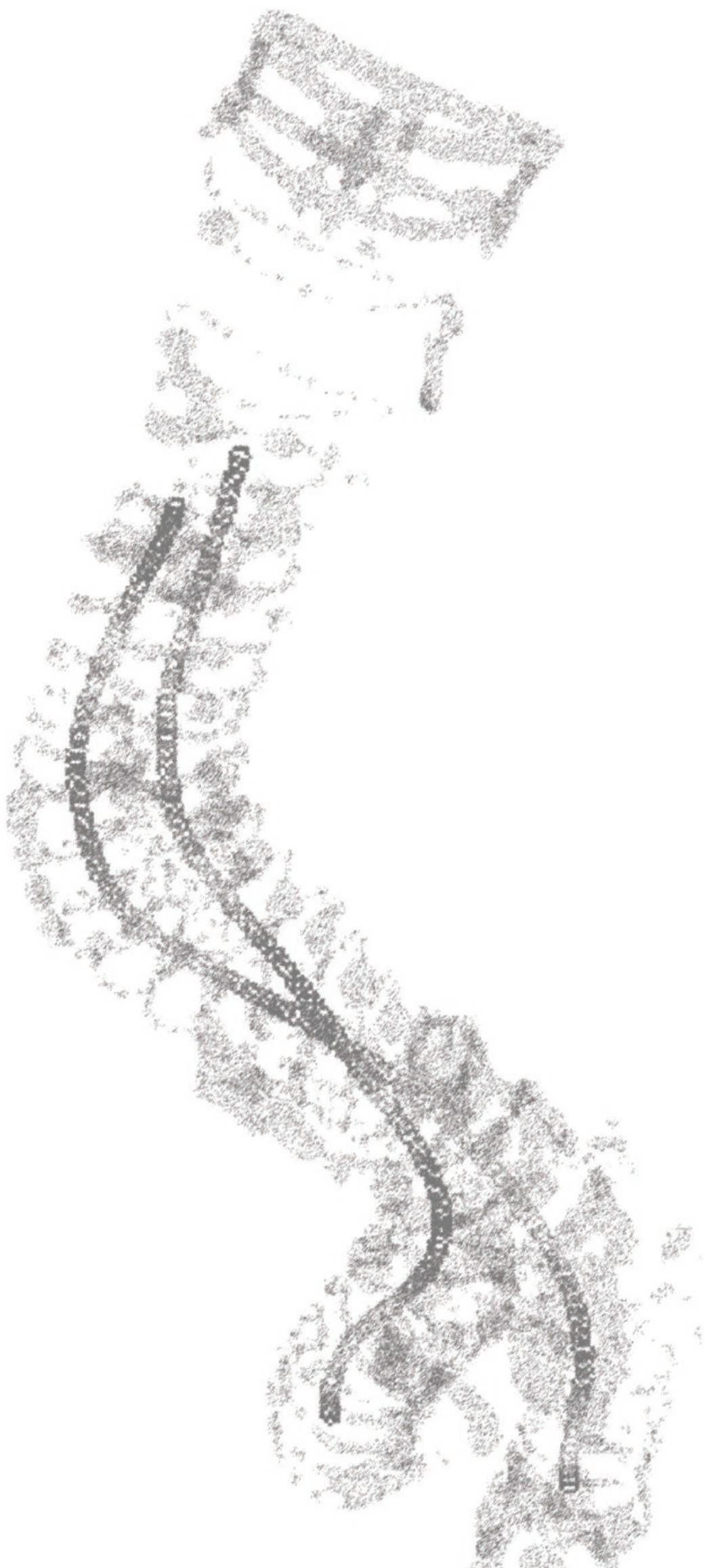





\section{Chapter 5}

\section{Geometric changes in Anaconda endograft limbs after endovascular aneurysm repair: A potential predictor for limb occlusion}

Jaimy A. Simmering ${ }^{a, b, *}$, Maaike A. Koenrades ${ }^{a, b, c, *}$, Cornelis H. Slump ${ }^{c}$, and Robert H. Geelkerken $^{a, b}$

* Jaimy A. Simmering and Maaike A. Koenrades contributed equally to this work and have shared first authorship

${ }^{a}$ Multi-modality Medical Imaging (M3I) group, Faculty of Science and Technology, Technical Medical Centre, University of Twente, Enschede, the Netherlands

${ }^{b}$ Department of Vascular Surgery, Medisch Spectrum Twente, Enschede, the Netherlands

${ }^{c}$ Robotics and Mechatronics (RaM) group, Faculty of Electrical Engineering, Mathematics and Computer Science, Technical Medical Centre, University of Twente, Enschede, the Netherlands

Preliminary data from this study were presented at the ESCVS International Congress (May 22-25, 2019; Groningen, the Netherlands)

Submitted 


\section{Abstract}

Objective: The emergence of limb occlusion after endovascular aneurysm repair may be related to the ongoing interaction between the endograft configuration and the patient's anatomy. We aimed to provide detailed information about geometric changes of Anaconda endograft limbs during follow-up as well as during the cardiac cycle.

Methods: Fifteen patients (mean age 72.8 \pm 3.7 years; 14 men) underwent postoperative electrocardiogram-gated computed tomography (CT) scans according to a prospective study design between April 2014 and May 2017. Changes in curvature, length of the limbs, and distances between successive stent-rings (inter-ring distance) of the endograft limbs during a 2-year follow-up period were quantified using meticulous image processing methods involving image registration, centerline extraction, and model-based stent-ring segmentation.

Results: From discharge to 24 months, mean curvature increased significantly by $9.6 \mathrm{~m}^{-1}$ (SD, $11.1 ; 95 \%$ CI 3.4 to $15.8 \mathrm{~m}^{-1} ; \mathrm{p}=0.002$ ) for the right limbs and by 6.1 $\mathrm{m}^{-1}$ (SD, 9.4; 95\% CI 0.8 to $11.5 \mathrm{~m}^{-1} ; \mathrm{p}=0.21$ ) for the left limbs. The length of the right limbs decreased significantly by $9.5 \mathrm{~mm}$ (SD, 7.6; 95\% CI 3.5 to $15.6 \mathrm{~mm}$; $\mathrm{p}=0.002$ ); the length of the left limbs by $10.1 \mathrm{~mm}$ (SD, $5.1 ; 95 \%$ CI 5.9 to $14.2 \mathrm{~mm}$; $\mathrm{p}<0.001)$. The minimal inter-ring distance decreased by $0.36 \mathrm{~mm}$ (SD, $0.26 ; 95 \%$ CI 0.17 to $0.55 \mathrm{~mm} ; \mathrm{p}<0.001)$ for the right limbs and $0.35 \mathrm{~mm}(\mathrm{SD}, 0.19 ; 95 \%$ CI 0.21 to $0.49 \mathrm{~mm} ; \mathrm{p}<0.001)$ for the left limbs. Cardiac pulsatility-induced changes in curvature, limb length and inter-ring distance were negligible $(2 \%, 0.3 \%$ and $0.3 \%$, respectively).

Conclusion: We observed changes in the geometry of the Anaconda endograft limbs after EVAR up to 24 months follow-up, as seen by an increase in curvature, shortening of the limbs, and a corresponding decrease in inter-ring distance. These geometry changes could result in inwards folding of the graft fabric, which may relate to the emergence of limb occlusion. Further investigation of these metrics in a larger cohort involving patients with and without occlusions may allow to determine their predictive value.

Keywords: Endovascular aneurysm repair, limb occlusion, geometry, curvature, Anaconda endograft, fabric, ring stent 


\section{Introduction}

Progress in endovascular aortic aneurysm repair (EVAR) has been made by advances in endograft design and image-guided endovascular techniques. However, compared to open surgery the benefits of this less invasive procedure[1,2] are challenged by a considerable reintervention rate up to $20 \%$ in the first five years after EVAR due to complications including endoleak, device migration and limb occlusion[2-4]. Since its introduction in 1998, the Anaconda endograft (Terumo Aortic, Inchinnan, Scotland, UK) has evolved to a third-generation device (One-Lok) with an independent nitinol stent-ring design to optimize device conformability and compatibility with tortuous anatomy. The Anaconda design has demonstrated durable aneurysm exclusion in daily practice[5-7] with a low reported number of type I endoleaks $(3.5 \%$ vs. 4.2-8.5\% for other endografts[4]), type III endoleaks (0.6\% vs. $0-1.5 \%$ for other endografts[4]) and endograft migration (0.6\% vs. $0-0.9 \%$ for other endografts[4]). Still, a recent single-center study involving 110 elective patients with a One-Lok Anaconda device reported an overall limb occlusion rate of $6.4 \%$ after a mean follow-up of 47 months, while the incidence of iliac limb occlusion was estimated $5.6 \%$ in a recent meta-regression analysis involving 5454 patients[8]. These results indicate that further efforts may be needed to optimize the next generation of Anaconda limbs in order to reduce the incidence of limb occlusion. Previous studies have identified predisposing factors that increase the risk of occlusion, including significant angulation and calcification of the iliac arteries, excessive limb oversizing, small distal limb diameter, small external iliac artery diameter extension of the endograft limb into the external iliac artery, and kinking[7,9-13]. Albeit useful to help define specific treatment strategies, these factors do not help to select patients at risk during follow-up as they do not provide insight in the ongoing interaction between the endograft configuration and the patient's anatomy. Moreover, detailed information about changes in limb geometry several months to years after EVAR is lacking in the literature. Understanding these changes may help to identify potential predictors of limb occlusion and additionally to improve endograft design. We hypothesize that shrinkage of the aneurysm sac may result in changes in the initial configuration including changes in limb length and limb angulation. Due to the independent stent-ring configuration of the Anaconda limbs, these changes may lead to inwards folding of the endograft fabric, which may contribute to the emergence of limb embolization or thrombosis by inducing regions with static, recirculating and turbulent flow patterns[11,14]. Additionally, pulsatile blood flow may induce cyclic geometric changes in limb configuration, potentially affecting flow patterns. The aim of this work was to provide detailed information about geometric changes of Anaconda endograft limbs during follow-up as well as during the cardiac cycle.

\section{Methods}

This study used postoperative electrocardiogram (ECG)-gated computed tomography (CT) scans of 15 asymptomatic infrarenal AAA patients (mean age $72.8 \pm 3.7$ years; 14 men) who underwent elective EVAR with an Anaconda One-Lok endograft between April 2014 and May 2015. These patients were prospectively enrolled in 
an observational single center cohort study (Longitudinal Study of Pulsatility and Expansion in Aortic Stent-grafts [LSPEAS], Trialregister.nl identifier NTR4276) and were followed for 24 months after EVAR by ECG-gated CT scans according to a standardized protocol: before discharge and after 1, 6, 12 and 24 months follow-up. The study design, patient sample and image acquisition protocol have been reported in detail previously[15]. Additional patient characteristics related to the iliac anatomy and implanted endograft limbs are presented in Appendix A. Table S1. The CT scans were acquired during a single breath hold and were reconstructed into 10 phases to cover the cardiac cycle with a slice thickness of $1 \mathrm{~mm}$. The CT scans were performed without contrast administration to preclude nephrotoxic effects. Two of the 15 patients did not undergo the 24 months scan (non-AAA-related death and voluntary withdrawal).

The Anaconda device The Anaconda endograft design, characteristics and implant technique have been previously described in detail $[5,16,17]$. Here, we briefly describe the Anaconda limb characteristics. The limbs consist of multiple independent nitinol stent-rings that are sewn onto the woven polyester graft fabric, providing flexibility in tortuous iliac arteries. The One-Lok Anaconda bifurcate body has a standardized iliac gate diameter of $10.5 \mathrm{~mm}$ to facilitate the standard $12 \mathrm{~mm}$ proximal limb diameter. To improve body-limb combinations for patient-specific sizing the distal outflow configuration can be either tapered, straight, or flared with stent-ring diameters ranging from 10 to $23 \mathrm{~mm}$. The distance between the $12 \mathrm{~mm}$ stent-rings is $5.5 \mathrm{~mm}$. For a $10 \mathrm{~mm}$ tapered end the inter-ring distance is $4.5 \mathrm{~mm}$; for a flared end the inter-ring distance increases with increasing stent-ring diameter up to $10 \mathrm{~mm}$ for a $23 \mathrm{~mm}$ flared limb.

ECG-gated CT image processing and analysis The ECG-gated CT scans were processed using a previously developed algorithm that uses image registration to estimate motion and image segmentation to model geometry of the endograft's stentframe[18-20]. The purpose of the image registration was two-fold: 1) to obtain a deformation (i.e., vector) field for each reconstructed phase in the cardiac cycle in order to obtain a phase-averaged 3-dimensional (3D) volume for image segmentation and 2) to evaluate motion and deformation of the limb geometry during the cardiac cycle. By using deformable registration, the phase-averaged 3D volumes representing mid cardiac cycle were compensated for motion artefacts to allow for assessment of limb deformation. The image processing steps have been detailed previously[20]. The algorithm was further refined to evaluate curvature and length of the limbs by obtaining center lumen lines (CLLs; Figure 1A). Hereto, a threshold segmentation (>600 Hounsfield units) was performed to obtain a set of points that describe the stent-frame. The CLLs were extracted by calculating the path through the center of the respective set of points of the stent-frame by maximizing the distance to these points. The start of the CLL was defined at the level of the most proximal stent-ring in the limb; the end at the level of the most distal stent-ring. The CLLs comprise a set of consecutive points with a set distance of $1 \mathrm{~mm}$. All CLLs were visually inspected. To quantify distances between the stent-rings of the limbs (e.g., perceived increased risk of limb occlusion related to inward folding of graft fabric) we created 3D geometric 

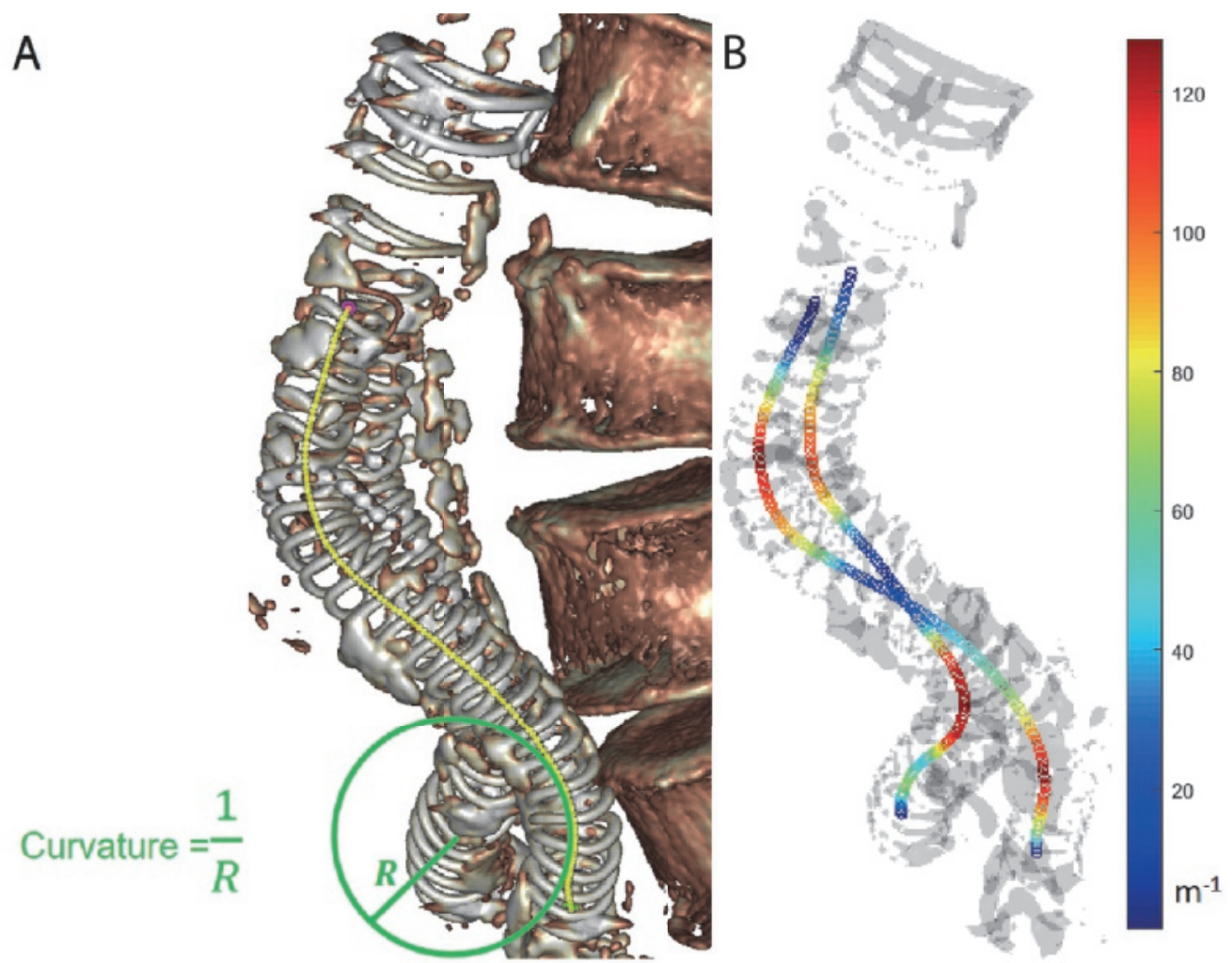

Figure 1. An example of the assessment of curvature over the center lumen line (CLL), illustrating (A) the calculation of curvature over the CLL along with (B) the color-coded curvature that was calculated for each point of the CLL of both limbs (blue represents low curvature, i.e., straight; red represents high curvature, i.e., angulated). R, radius of curvature.

models of each stent-ring. To obtain individual stent-ring models, seed points for initialization were manually selected per stent-ring, after which the stent-ring models were automatically obtained[19]. To evaluate cardiac-induced changes of the metrics, the CLLs and stent-ring models that were generated in the phase-averaged 3D volumes were transformed to the 10 phases of a cardiac cycle by backward mapping of the deformation fields. Applying this image registration algorithm instead of repeating the measurements in the different phases of the cardiac cycle is (1) time efficient and (2) avoids inconsistencies between the models and CLLs in the different phases that may drastically reduce the accuracy of the measurements.

Curvature Curvature was defined as the inverse of the radius of the spherical shape that best approximates the curve at each point along the CLL, expressed in $\mathrm{m}^{-1}$ (Figure 1). Mathematically, curvature was calculated for a parametrically-defined $3 \mathrm{D}$ curve (equation 1$)$ : 


$$
\kappa=\frac{\sqrt{\left(z^{\prime \prime} y^{\prime}-y^{\prime \prime} z^{\prime}\right)^{2}+\left(x^{\prime \prime} z^{\prime}-z^{\prime \prime} x^{\prime}\right)^{2}+\left(y^{\prime \prime} x^{\prime}-x^{\prime \prime} y^{\prime}\right)^{2}}}{\left(x^{\prime 2}+y^{\prime 2}+z^{\prime 2}\right)^{3 / 2}},
$$

where $x(n), y(n)$ and $z(n)$ are the Cartesian coordinates in three dimensions for the number of points $n$ along the CLL, ' is the first derivative and " the second derivative. The curvature calculation was implemented in Matlab 2018a (The Mathworks, Natick, MA, USA) and was validated using a helix-shaped phantom that was designed to validate curvature calculations[21]. Larger curvature values indicate a stronger limb angulation, i.e., larger limb curvature. Change in mean and maximum curvature during follow-up was assessed for each limb by comparing these metrics at mid-cardiac cycle. Furthermore, mean and maximal cardiac pulsatility-induced changes in curvature were evaluated in each scan. Maximal change in curvature indicated the largest change in curvature at a particular CLL point and mean change indicates the average change of all CLL points.

Limb length The length of the endograft limbs was assessed as the length of the CLLs in mm. The change in limb length was evaluated over time at mid-cardiac cycle and in each scan during the cardiac cycle by comparing the limb length in each phase of the cardiac cycle.

Distances between stent-rings Distances were calculated between successive stent-rings, i.e., inter-ring distances. For each pair of successive stent-rings the shortest distance from each point on the most cranial stent-ring to a point on the successive stent-ring was automatically obtained. These distances were used to calculate mean and minimal inter-ring distance for each ring pair (expressed in mm; Figure 2). For each limb, the mean and minimal inter-ring distance were calculated by respectively taking the average of the mean and minimal inter-ring distances of all ring pairs. Change in mean and minimal inter-ring distance during follow-up was assessed by comparing these metrics at mid cardiac cycle. Again, cardiac-induced changes in distance were evaluated in each scan.

Statistical analysis Normality of the data was checked by histograms. The data was found to be normally distributed and was displayed as mean values followed by the standard deviation (SD) and the $95 \%$ confidence interval (CI) in brackets. Mixedmodel repeated measures analysis was performed to assess changes in curvature, limb length and inter-ring distances during follow-up. A fixed effects model was used to evaluate differences in change between the right and left limb at different time points relative to discharge with the assumption that changes in the right and left limbs were unrelated. The optimal covariance type was selected per analysis based on Akaike's Information Criterion. A post-hoc Sidak correction was used to correct for multiple comparisons. The two-tailed Pearson's correlation coefficient (PCC) was used to investigate the relationship between different metrics. For all analyses a significance level of $\mathrm{p}<0.05$ was assumed statistically significant. Statistical analyses were performed using SPSS Statistics version 25.0 (IBM Corporation, Armonk, NY, USA). 


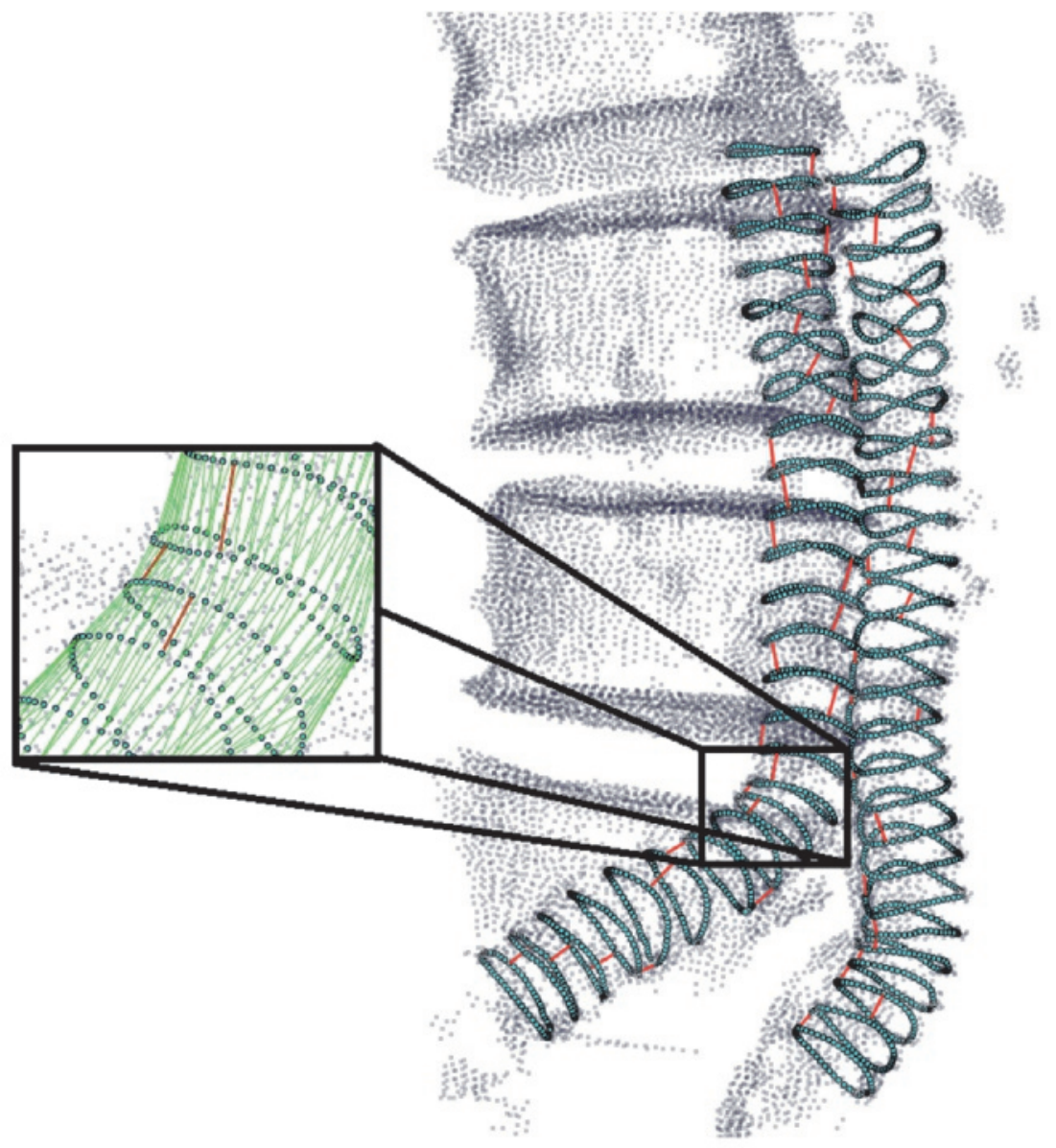

Figure 2. Example of the assessment of inter-ring distances between successive stent-rings. The 3D geometric models of the individual stent-rings are shown for which distances between the points on the successive stent-rings were automatically calculated (green lines in the boxmagnification). The minimal distance between each pair of stent-rings is indicated with red lines. 
Table 1. Evolution of geometric changes in the Anaconda endograft limbs through the 24-month follow-up period. ${ }^{a}$

\begin{tabular}{|c|c|c|c|c|c|}
\hline & $\begin{array}{l}\text { Discharge } \\
(\mathrm{n}=15)\end{array}$ & $\begin{array}{c}1 \text { month } \\
(\mathrm{n}=15)\end{array}$ & $\begin{array}{c}6 \text { months } \\
(\mathrm{n}=15)\end{array}$ & $\begin{array}{l}12 \text { months } \\
(\mathrm{n}=15)\end{array}$ & $\begin{array}{l}24 \text { months } \\
(\mathrm{n}=13)\end{array}$ \\
\hline $\begin{array}{l}\text { Mean curvature, } \\
\mathrm{m}^{-1}\end{array}$ & $\begin{array}{c}18.88 \pm 4.43 \\
(17.23- \\
20.54)\end{array}$ & $\begin{array}{c}19.35 \pm 5.06 \\
(17.45- \\
21.24)\end{array}$ & $\begin{array}{c}21.89 \pm 6.58 \\
(19.43- \\
24.35)\end{array}$ & $\begin{array}{c}24.85 \pm 7.17 \\
(22.17- \\
27.53)\end{array}$ & $\begin{array}{c}27.02 \pm 8.71 \\
(23.76- \\
30.28)\end{array}$ \\
\hline $\begin{array}{l}\triangle \text { curvature, } \\
\mathrm{m}^{-1}\end{array}$ & - & $\begin{array}{c}0.46 \pm 1.89 \\
(-0.45- \\
1.38) \\
\mathrm{p}=0.568\end{array}$ & $\begin{array}{c}3.00 \pm 4.00 \\
(1.06-4.95) \\
\mathrm{p}=0.001\end{array}$ & $\begin{array}{c}5.96 \pm 5.35 \\
(-3.37- \\
8.56) \\
\mathrm{p}<0.001\end{array}$ & $\begin{array}{c}8.14 \pm 7.18 \\
(4.38- \\
11.90) \\
\mathrm{p}<0.001\end{array}$ \\
\hline $\begin{array}{l}\text { Maximal curvature, } \\
\mathrm{m}^{-1}\end{array}$ & $\begin{array}{c}57.69 \pm 25.22 \\
(48.24- \\
67.14)\end{array}$ & $\begin{array}{c}57.54 \pm 25.72 \\
(47.87- \\
67.20)\end{array}$ & $\begin{array}{c}58.80 \pm 23.78 \\
(49.76- \\
67.84)\end{array}$ & $\begin{array}{c}67.06 \pm 26.71 \\
(56.98- \\
77.13)\end{array}$ & $\begin{array}{c}74.45 \pm 29.56 \\
(64.69- \\
86.44)\end{array}$ \\
\hline $\begin{array}{l}\triangle \text { curvature } \\
\mathrm{m}^{-1}\end{array}$ & - & $\begin{array}{c}-0.15 \pm 8.95 \\
(-4.50- \\
4.20) \\
\mathrm{p}>0.99\end{array}$ & $\begin{array}{c}1.11 \pm 16.05 \\
(-6.69- \\
8.91) \\
p=0.993\end{array}$ & $\begin{array}{c}9.37 \pm 22.69 \\
(-1.66- \\
20.39) \\
p=0.121\end{array}$ & $\begin{array}{c}17.88 \pm 26.49 \\
(4.04- \\
31.72) \\
\mathrm{p}=0.007\end{array}$ \\
\hline $\begin{array}{l}\text { Maximal curvature } \\
\text { location, } \mathrm{mm}\end{array}$ & $\begin{array}{c}103.9 \pm 43.8 \\
(87.1- \\
118.8)\end{array}$ & $\begin{array}{c}101.5 \pm 46.7 \\
(85.7- \\
117.4)\end{array}$ & $\begin{array}{c}96.4 \pm 42.7 \\
(80.52- \\
112.2)\end{array}$ & $\begin{array}{c}85.2 \pm 48.2 \\
(69.4- \\
101.0)\end{array}$ & $\begin{array}{c}66.9 \pm 39.7 \\
(57.1-89.6)\end{array}$ \\
\hline $\begin{array}{l}\triangle \text { location, } \\
\mathrm{mm}\end{array}$ & - & $\begin{array}{c}-1.4 \pm 28.57 \\
(-14.5- \\
11.7) \\
p=0.998\end{array}$ & $\begin{array}{c}-6.6 \pm 32.1 \\
(-21.4-8.3) \\
p=0.711\end{array}$ & $\begin{array}{c}-17.7 \pm 36.2 \\
(-34.6--0.7) \\
p=0.038\end{array}$ & $\begin{array}{c}-29.6 \pm 35.0 \\
(-47.7- \\
-11.5) \\
p=0.001\end{array}$ \\
\hline $\begin{array}{l}\text { Limb length, } \\
\mathrm{mm}\end{array}$ & $\begin{array}{c}147.5 \pm 30.5 \\
(136.0- \\
159.0)\end{array}$ & $\begin{array}{c}145.7 \pm 30.0 \\
(134.4- \\
157.0)\end{array}$ & $\begin{array}{c}142.1 \pm 30.3 \\
(130.7- \\
153.6)\end{array}$ & $\begin{array}{c}140.5 \pm 30.3 \\
(129.1- \\
151.9)\end{array}$ & $\begin{array}{c}132.9 \pm 30.3 \\
(125.7- \\
150.2)\end{array}$ \\
\hline $\begin{array}{l}\triangle \text { length, } \\
\mathrm{mm}\end{array}$ & - & $\begin{array}{c}-1.8 \pm 1.5 \\
(-2.6--1.1) \\
\mathrm{p}<0.001\end{array}$ & $\begin{array}{c}-5.4 \pm 3.6 \\
(-7.1--3.6) \\
p<0.001\end{array}$ & $\begin{array}{c}-7.1 \pm 4.7 \\
(-9.3--4.8) \\
\mathrm{p}<0.001\end{array}$ & $\begin{array}{c}-9.6 \pm 6.8 \\
(-13.2--6.0) \\
p<0.001\end{array}$ \\
\hline $\begin{array}{l}\text { Mean inter-ring } \\
\text { distance, } \mathrm{mm}\end{array}$ & $\begin{array}{c}4.67 \pm 0.33 \\
(4.54-4.80)\end{array}$ & $\begin{array}{c}4.65 \pm 0.35 \\
(4.52-4.78)\end{array}$ & $\begin{array}{c}4.61 \pm 0.34 \\
(4.48-4.74)\end{array}$ & $\begin{array}{c}4.56 \pm 0.35 \\
(4.43-4.69)\end{array}$ & $\begin{array}{c}4.49 \pm 0.39 \\
(4.36-4.63)\end{array}$ \\
\hline $\begin{array}{l}\triangle \text { distance, } \\
\mathrm{mm}\end{array}$ & - & $\begin{array}{c}-0.02 \pm 1.48 \\
(-0.09- \\
0.05) \\
\mathrm{p}=0.956\end{array}$ & $\begin{array}{c}-0.06 \pm 1.48 \\
(-0.13- \\
0.01) \\
p=0.089\end{array}$ & $\begin{array}{c}-0.11 \pm 1.48 \\
(-0.18- \\
-0.05) \\
p<0.001\end{array}$ & $\begin{array}{c}-0.17 \pm 1.43 \\
(-0.25- \\
-0.10) \\
p<0.001\end{array}$ \\
\hline $\begin{array}{l}\text { Minimal inter-ring } \\
\text { distance, } \mathrm{mm}\end{array}$ & $\begin{array}{c}3.71 \pm 0.42 \\
(3.55-3.86)\end{array}$ & $\begin{array}{c}3.72 \pm 0.43 \\
(3.56-3.87)\end{array}$ & $\begin{array}{c}3.64 \pm 0.37 \\
(3.49-3.79)\end{array}$ & $\begin{array}{c}3.55 \pm 0.37 \\
(3.40-3.70)\end{array}$ & $\begin{array}{c}3.39 \pm 0.42 \\
(3.20-3.50)\end{array}$ \\
\hline $\begin{array}{l}\triangle \text { distance, } \\
\mathrm{mm}\end{array}$ & - & $\begin{array}{c}0.01 \pm 2.46 \\
(-0.11- \\
0.12) \\
p=0.999\end{array}$ & $\begin{array}{c}-0.07 \pm 2.46 \\
(-0.18- \\
0.05) \\
p=0.485\end{array}$ & $\begin{array}{c}-0.16 \pm 2.46 \\
(-0.27- \\
-0.04) \\
p=0.003\end{array}$ & $\begin{array}{c}-0.36 \pm 2.40 \\
(-0.48- \\
-0.24) \\
\mathrm{p}<0.001\end{array}$ \\
\hline
\end{tabular}

${ }^{a}$ Measurements are presented as mean \pm SD with $95 \%$ CI in brackets; Change indicates the difference to discharge. 
A
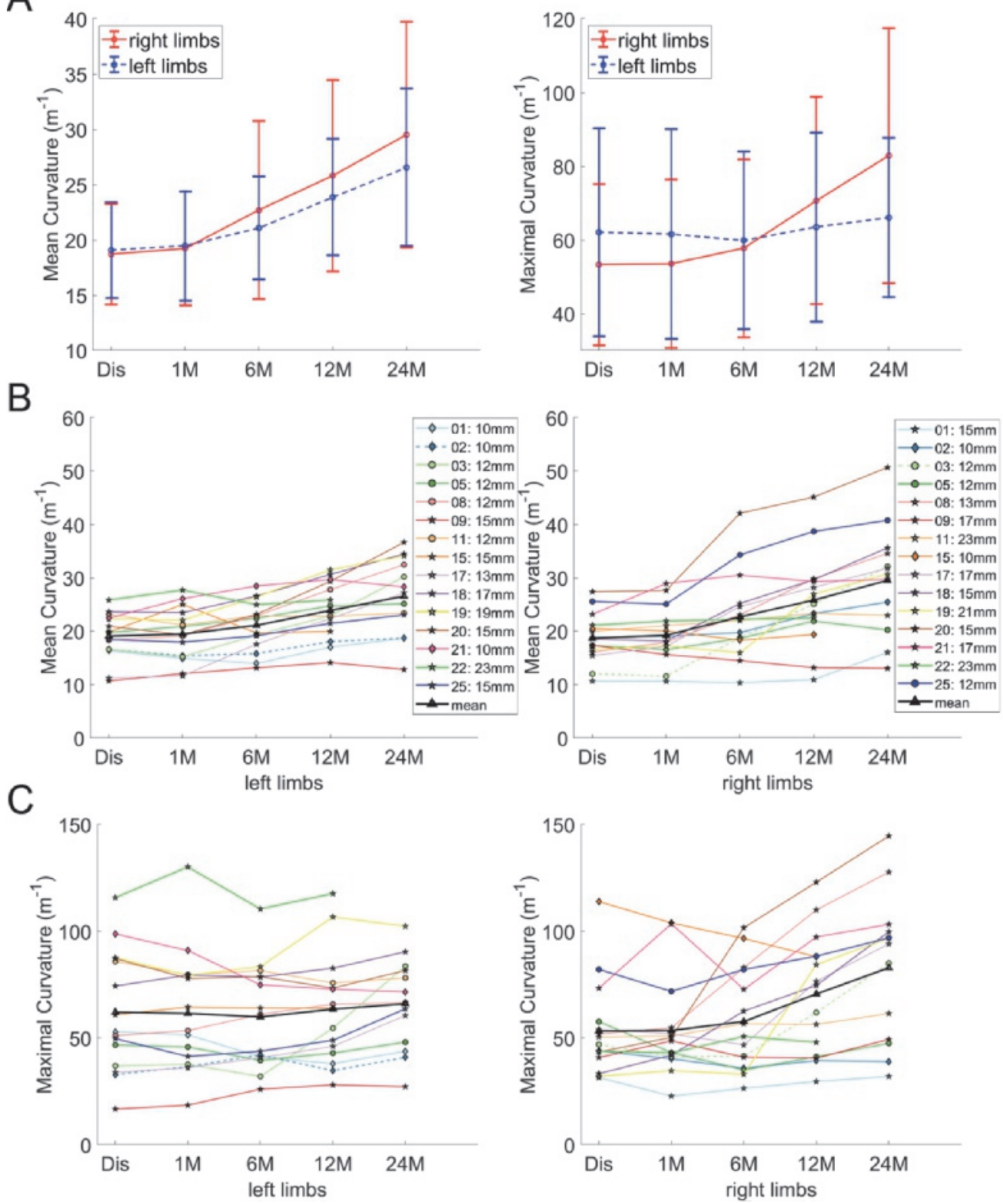

Figure 3. Evolution of mean and maximal curvature of the left and right limbs of the Anaconda endograft from discharge to 24 months EVAR. (A) The mean (dot) and standard deviation (whiskers) are displayed as well as the (B) values of the mean and (C) maximal curvature for each individual patient. The distal diameters of the limbs are shown for each patient in the legend, where three type of markers indicates tapered $(\diamond)$, straight $(\mathrm{o})$, or flared $(\star)$. Dis, discharge; M, months after EVAR. 
A
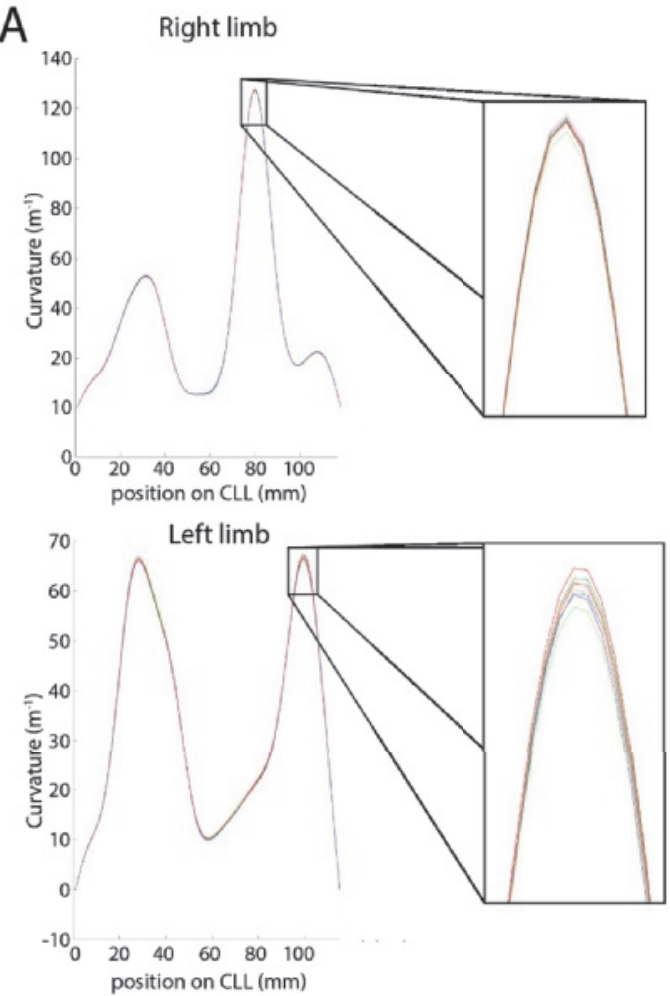

B

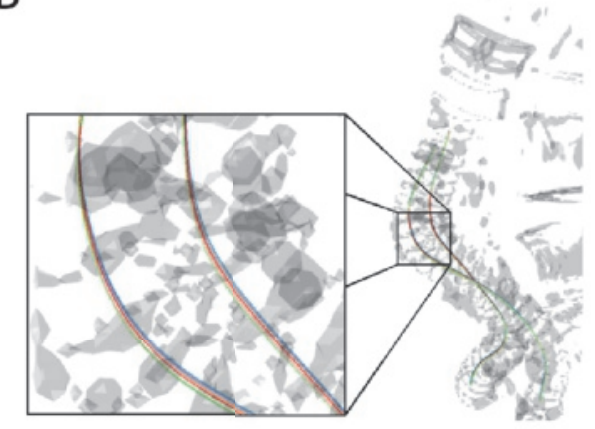

C

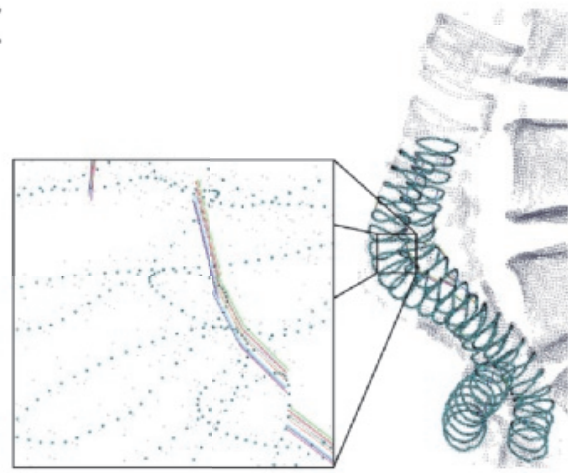

Figure 4. An example of cardiac pulsatility-induced changes in limb geometry in one patient. (A) Curvature along the CLL for the 10 different phases in a cardiac cycle; points on the CLL are in consecutive order, starting at the proximal point of the CLL. (B) Threedimensional view of the CLLs in each phase in a cardiac cycle. (C) Three-dimensional view of the stent-ring models at mid-cardiac cycle with the minimal inter-ring distances shown for each phase in a cardiac cycle. Each line represents one cardiac phase.

\section{Results}

Table 1 presents geometric changes in curvature, limb length and inter-ring distance in the Anaconda endograft left and right limbs through the 24-month follow-up period.

Curvature Figure 3 presents the change in mean and maximal curvature from discharge to 24 months after EVAR. For all patients, mean and maximal curvature of both limbs had increased over time. From discharge to 24 months, mean curvature increased significantly by $9.6 \mathrm{~m}^{-1}$ (SD, $11.1 ; 95 \%$ CI 3.4 to $15.8 \mathrm{~m}^{-1}$; $\mathrm{p}=0.002$ ) for the right limbs and by $6.1 \mathrm{~m}^{-1}\left(\mathrm{SD}, 9.4 ; 95 \%\right.$ CI 0.8 to $\left.11.5 \mathrm{~m}^{-1} ; \mathrm{p}=0.21\right)$ for the left limbs. Maximal curvature increased significantly for the right limbs by $28.3 \mathrm{~m}^{-1}$ (SD, $32.2 ; 95 \%$ CI 3.0 to $\left.53.6 \mathrm{~m}^{-1} ; \mathrm{p}=0.025\right)$, but not for the left limbs $\left(7.7 \mathrm{~m}^{-1}\right.$; SD, 22.4 ; $95 \%$ CI -4.5 to $\left.20.0 \mathrm{~m}^{-1} ; \mathrm{p}=0.330\right)$. On average, this is an increase of $56 \%$ (SD $46 \%$ ) of the mean curvature measured at discharge and an increase of $52 \%$ (SD $73 \%$ ) of 
the maximal curvature. The change in curvature from discharge to 24 months of the left and right limbs was not significantly different for mean $(\mathrm{p}=0.294)$ nor maximal curvature $(\mathrm{p}=0.060)$.

The location of maximal curvature on the CLL, measured from the proximal end, moved cranially during the 24-month follow-up period for both the right and the left limbs (right: $25.0 \mathrm{~mm}$, SD 40.5, 95\% CI -1.8 to $51.8 \mathrm{~mm}, \mathrm{p}=0.072$; left: $33.9 \mathrm{~mm}$, SD $31.9,95 \%$ CI 9.3 to $58.5 \mathrm{~mm}, \mathrm{p}=0.005)$. There was no significant difference between both limbs $(\mathrm{p}=0.573)$, resulting in an overall shifting from $103.9 \mathrm{~mm}$ (SD, 43.8; $95 \%$ CI 87.1 to $118.8 \mathrm{~mm}$ ) to $66.9 \mathrm{~mm}$ (SD, 39.7; $95 \%$ CI 57.1 to $89.6 \mathrm{~mm}$ ) from the proximal end of the CLL by a mean change of $29.6 \mathrm{~mm}$ (SD, 35.0; 95\% CI 11.5 to 47.7; $\mathrm{p}=0.001)$.

During the cardiac cycle, the mean and maximal change in curvature ranged from 0.0292 to $1.497 \mathrm{~m}^{-1}$ and from 0.493 to $4.167 \mathrm{~m}^{-1}$, respectively. These changes are on average $1.2 \%$ and $2.2 \%$ of respectively the smallest mean and maximal curvature values during the cardiac cycle. An example of cardiac pulsatility-induced changes in CLL geometry and curvature is shown in Figure 4A,B. The changes in mean and maximal curvature during the cardiac cycle did not significantly differ between the right and left limbs $(\mathrm{p}>0.422)$ nor between time points during follow-up ( $>>0.096)$.

Limb length The length of the limbs decreased significantly from discharge to 24 months by $9.6 \mathrm{~mm}$ (SD, 6.8; 95\% CI 6.0 to $13.2 \mathrm{~mm}$; $<<0.001$ ). Right limbs decreased in length by $9.5 \mathrm{~mm}$ (SD, 7.6; $95 \%$ CI 3.5 to $15.6 \mathrm{~mm} ; \mathrm{p}=0.002$ ) and left limbs by $10.1 \mathrm{~mm}$ (SD, 5.1; $95 \%$ CI 5.9 to $14.2 \mathrm{~mm}$; $<0.001$; Figure 5). On average, this is a decrease of $7.6 \%$ (SD $5.0 \%$ ) of the length at discharge. No significant difference in change in limb length was observed between right and left limbs $(p=0.877)$.

Change in limb length during cardiac cycle ranged from 0.09 to $1.22 \mathrm{~mm}$ and was not statistically different between the right and left limbs $(p=0.894)$. On average, the cardiac pulsatility-induced changes were $0.3 \%$ of the smallest limb length during the cardiac cycle. During follow-up, these changes were statistically different between discharge and 1 month only (-0.100 vs $-0.006 \mathrm{~mm}, \mathrm{p}=0.033)$.

Distances between rings A decrease was observed in the mean inter-ring distance from discharge to 24 months (all limbs: $0.17 \mathrm{~mm}$, SD $0.14,95 \%$ CI 0.10 to $0.25 \mathrm{~mm}$, $\mathrm{p}<0.001$; right: $0.17 \mathrm{~mm}$, SD 0.17, 95\% CI -0.04 to $0.29 \mathrm{~mm}, \mathrm{p}=0.004$; left: $0.18 \mathrm{~mm}$, SD $0.14,95 \%$ CI 0.11 to $0.25 \mathrm{~mm}, \mathrm{p}<0.001$; Figure 6 ). Similarly, the minimal interring distance decreased by $0.36 \mathrm{~mm}$ (SD, $0.24 ; 95 \%$ CI 0.24 to $0.48 \mathrm{~mm} ; \mathrm{p}<0.001$ ) for all limbs, $0.36 \mathrm{~mm}$ (SD, 0.26; 95\% CI 0.17 to $0.55 \mathrm{~mm} ; \mathrm{p}<0.001)$ for the right limbs, and $0.35 \mathrm{~mm}$ (SD, $0.19 ; 95 \%$ CI 0.21 to $0.49 \mathrm{~mm}$; $<<0.001$ ) for the left limbs (Figure $6)$. These differences between the right and left limbs were not statistically significant $(\mathrm{p}>0.763)$.

Cardiac pulsatility-induced changes in mean and minimal inter-ring distances ranged from 0.004 to $0.030 \mathrm{~mm}$ and from 0.004 to 0.021 , respectively. This is on average $0.3 \%$ of the smallest minimal inter-ring distances during the cardiac cycle. Figure $4 \mathrm{C}$ shows an example of cardiac induced changes in minimal inter-ring distances. During follow-up, these changes did not differ between time points $(\mathrm{p}>0.082)$, except 

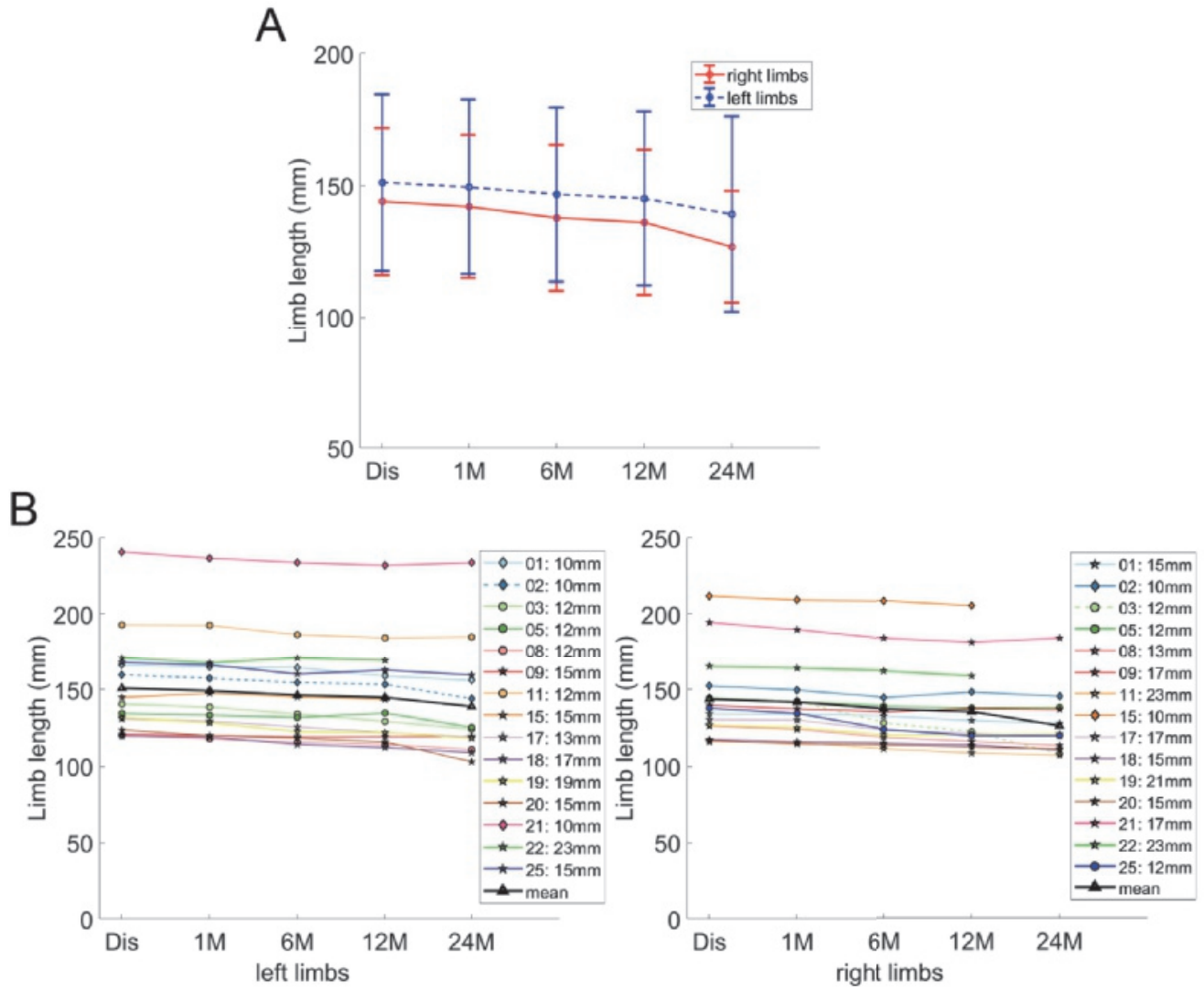

Figure 5. Evolution in limb length of the left and right limbs of the Anaconda endograft from discharge to 24 months after EVAR. (A) The mean (dot) and standard deviation (whiskers) are displayed as well as (B) the limb length for each individual patient. The distal diameters of the limbs are shown for each patient in the legend, where three type of markers indicates tapered $(\diamond)$, straight $(o)$, or flared $(\star)$. Dis, discharge; M, months after EVAR.

for minimal inter-ring distance between discharge and 1 month follow-up $(0.010 \mathrm{~mm}$, SD $0.004 \mathrm{~mm}$ vs. 0.008 , SD $0.003 \mathrm{~mm}, \mathrm{p}=0.038)$.

Correlations between metrics The change in mean curvature during the 24months follow-up period correlated with the change in minimal inter-ring distance $(\mathrm{PCC}=-0.63, \mathrm{p}=0.001)$ and the change in limb length $(\mathrm{PCC}=-0.47, \mathrm{p}=0.016) . \mathrm{Ad}-$ ditionally, the change in limb length correlated positively with the change in minimal inter-ring distance during 24 months follow up $(\mathrm{PCC}=0.86, \mathrm{p}<0.001)$. Furthermore, the 24-month change in aneurysm sac diameter significantly correlated with the change in limb length $(\mathrm{PCC}=0.43, \mathrm{p}=0.028)$ and minimal inter-ring distances $(\mathrm{PCC}=0.41, \mathrm{p}=0.039)$, but not with the change in mean curvature $(\mathrm{PCC}=-0.31$, $\mathrm{p}=0.121)$. 
Patients with limb occlusion In two of the 15 patients, unilateral limb occlusions were observed 27 months after EVAR (patient \#2 left limb; patient \#3 right limb). The limb occlusions were treated by endovascular recanalization (thrombectomy) and endolining (10 mm Advanta V12 balloon-expandable covered stents, Maquet Getinge Group, Hudson, NH, USA). Patency remained to date, 3 years after reintervention. For these patients, the metrics considered here (curvature, limb length and inter-ring distance) are shown as dotted lines in Figures 3,5 and 6.

\section{Discussion}

The present study evaluated in detail geometrical changes in Anaconda endograft limbs over time and during cardiac cycle by quantifying curvature of the limbs, limb length and distances between successive stent-rings in the limbs (inter-ring distance). During 24-months follow-up, curvature increased and limb length and inter-ring distances decreased. Change in minimal inter-ring distance correlated with the change in mean curvature, limb length and AAA sac diameter, which suggests that these metrics may be related to the emergence of limb occlusion by inwards folding of the graft fabric as the inter-ring distance decreases. Another relevant finding of this study is that geometry changes per heartbeat in curvature, limb length and inter-ring distance were negligible $(2 \%, 0.3 \%$ and $0.3 \%$, respectively).

Recently, Rödel et al.[7] observed a $6.4 \%$ limb occlusion rate in the third generation device compared to a $13.1 \%$ limb occlusion rate in the second generation device at a mean follow-up of 47 months. This suggests that there is room for improvement as the average rate of limb occlusion was reported $5.6 \%[8]$. Iliac limb angulation $\geq 60^{\circ}$, distal limb diameter $\leq 13 \mathrm{~mm}$, iliac calcification and extension of the limb (into the external iliac artery) are known predisposing factors for limb occlusion $[7,9,10,12,13]$. However, limb occlusion emerges also in patients without these predisposing factors and occlusions may be related to post-EVAR changes in limb configuration as observed in the present study. Shrinkage of the AAA sac, which occurs also longitudinally[22], seems to cause a shortening of the limb length and a decrease in inter-ring distance. Shortening of the limbs may also form regions with increased curvature, which could explain the observation that the location of maximum curvature shifts cranially into the AAA sac. Inwards folding of the graft fabric and increased angulation can result in regions of fluid stasis and high shear stress, which are known to increase the platelet activation potential and thus increase the risk of thrombus formation[23].

The limited changes during the cardiac cycle are not an unexpected finding since the difference between the arterial pressure inside the stent-graft and the AAA sac pressure outside the stent-graft is small throughout the cardiac cycle due to a lack of pulse wave in the sac that even decreases further over time[24]. In addition, aortic distension after EVAR at the level of maximum AAA diameter was previously observed to be small per cardiac cycle[25,26] as well as variations in stress and strain in limbs of various EVAR endografts between systole and diastole[27]. Still, the observed cardiac pulsatility-induced changes in curvature are slightly higher than the curvature change reported by Itoga et al.[28] for the Nellix device (Endologix Inc, Irvine, CA, USA). This is probably due to the difference in stent-graft design where 
A
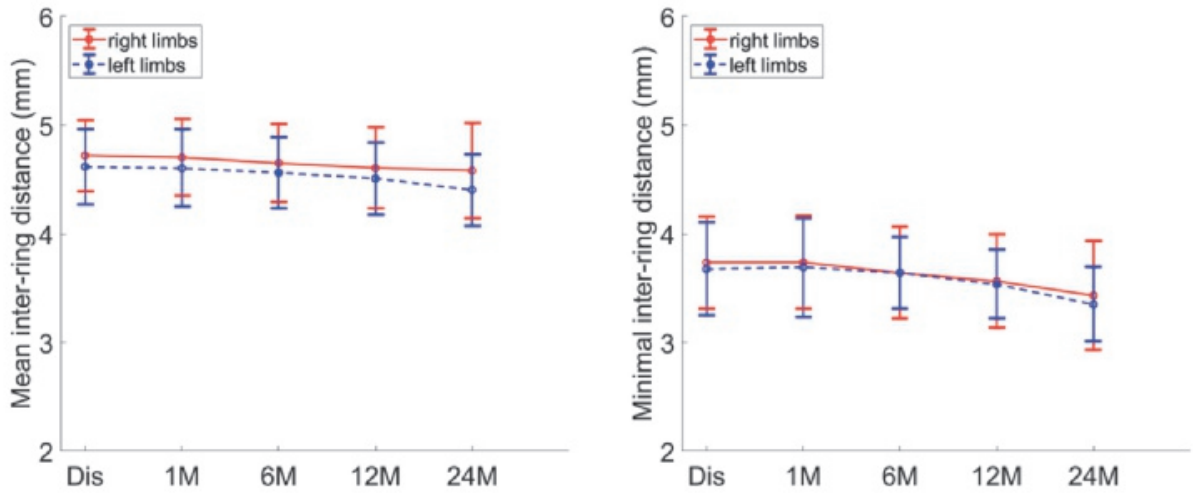

B
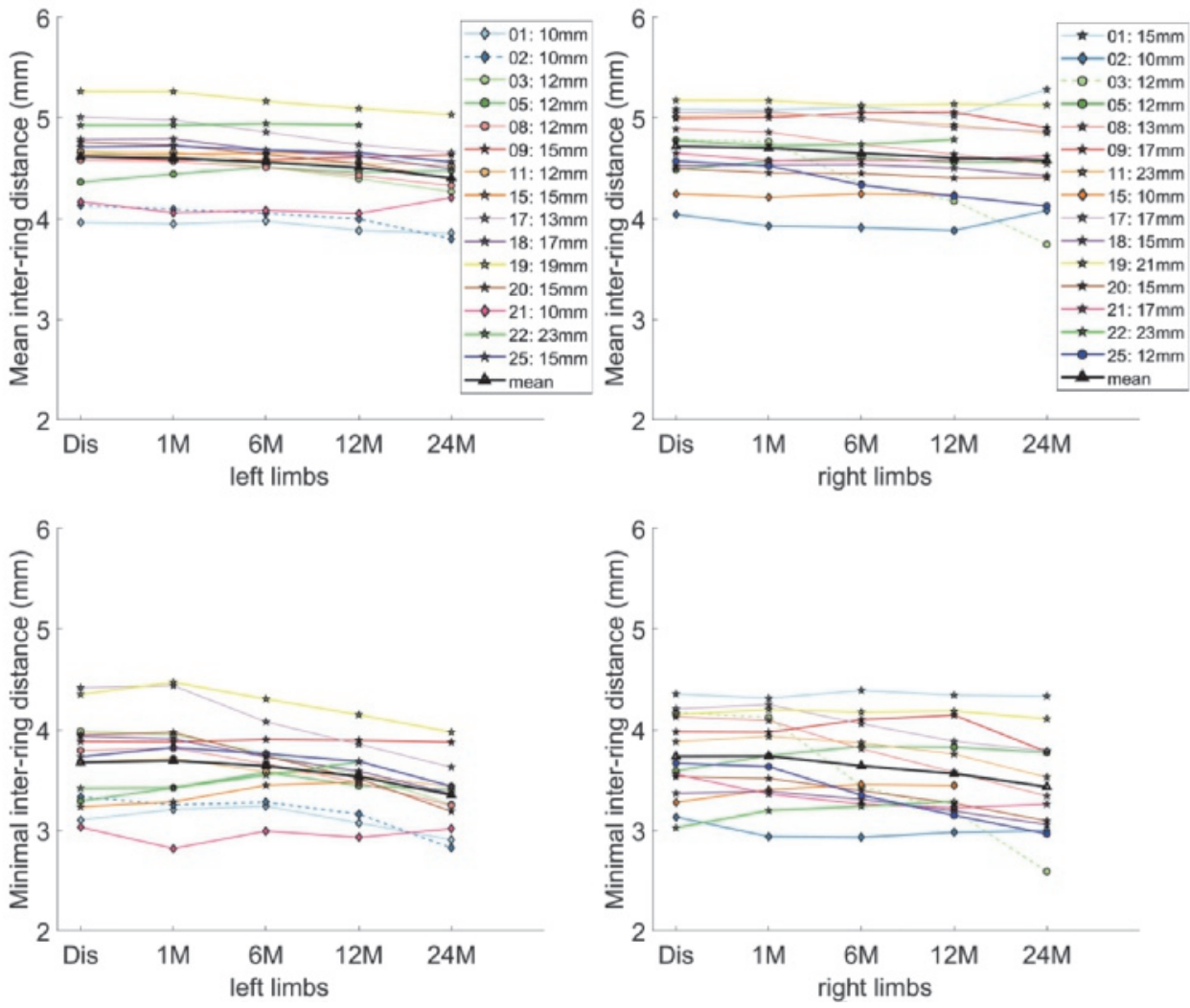

Figure 6. Evolution of mean and minimal inter-ring distance of the left and right limbs of the Anaconda endograft from discharge to 24 months after EVAR. (A) The mean (dot) and standard deviation (whiskers) are displayed as well as (B) the values of the mean and (C) minimal inter-ring distances for each individual patient. The distal diameters of the limbs are shown for each patient in the legend, where three type of markers indicates tapered $(\diamond)$, straight $(\mathrm{o})$, or flared $(\star)$. Dis, discharge; M, months after EVAR. 
the Nellix device uses stiff balloon-expandable stent-grafts while the Anaconda device has flexible self-expanding stent-graft limbs with lower column strength.

Notably, two of the 15 patients developed limb occlusion 27 months after EVAR. We observe several interesting differences compared to the other patients. In one patient (\#3), the occluded limb shows an increase in curvature far above average and the largest decrease in limb length and inter-ring distance. The other patient (\#2) shows an average increase in curvature but presents a small inter-ring distance from discharge on with an apparent decrease in limb length and mean inter-ring distance from 12 to 24 months. Minimal inter-ring distance decrease was even perceivable from 6 months on. In both patients, the inter-ring distances of the occluded limb were the smallest of all limbs at 24 months follow-up, i.e., shortly before they were diagnosed with limb occlusion. In addition, these two patients have relatively small distal limb diameters ( $\leq 12 \mathrm{~mm}$ ), for which the incidence of limb occlusion appears to be higher[7]. While other limbs also showed small inter-ring distances similar to the occluded limbs, in those cases the inter-ring distances did not decrease, except in one patient ( $\# 1$, left limb) who based on these observations might be at risk to develop limb occlusion. However, the relation of the geometric changes considered in the present study, complementary to known predisposing factors, should be further investigated in a larger cohort comprising patients that have and have not presented limb occlusion.

Finally, it should be noted that the initial mean inter-ring distance was always below the fabricated $5.5 \mathrm{~mm}$ straight limb inter-ring distance. This implies that the limbs may not have been deployed to their full length, possibly due to overestimation of the required limb length, which is not uncommon, especially in angulated anatomies[29]. Also, extension of the limbs (12 of the 30 limbs were extended) may lead to introduction of limbs that exceed the length of the iliac anatomy and consequently upward pushing of the limbs during intervention, inducing smaller inter-ring distances. Therefore, we recommend to measure the length of device limb required three-dimensionally and to stretch out the limbs during intervention in order to prevent inwards folding of graft fabric due to small inter-ring distances.

The present study involved a relatively small patient cohort, which may be considered a limitation. This is related to our local regular EVAR follow-up protocol that comprises duplex ultrasound instead of CT examinations. Nevertheless, the ECG-gated CT scans, prospective study design with close follow-up and meticulous processing methods allowed for in depth evaluation of both geometry changes during follow-up and changes due to pulsatile blood flow, which cannot be achieved in static CT scans. Furthermore, it should be noted that the small cardiac pulsatility-induced changes approached the experimentally derived detection limit of $0.3 \mathrm{~mm}[20]$. Finally, because modelling the individual stent-rings is currently time-consuming the methodology should be further optimized in order to use the measurement of interring distances in clinical practice.

\section{Conclusion}

The findings of this study suggest that postoperatively the geometry of the Anaconda limbs changes over time as seen by an increase in curvature, shortening of the limbs, 
and a corresponding decrease in distance between successive stent-rings, particularly in two patients that later on were diagnosed with limb occlusion. As these geometry changes could result in inwards folding of the graft fabric, the metrics considered in the present study may be related to the emergence of limb occlusion. Examining these metrics in a larger cohort involving patients with and without occlusions may allow to determine their predictive value.

\section{Acknowledgments}

The authors thank Prof. Dr. J.A.M. van der Palen (clinical epidemiologist, Medisch Spectrum Twente/University of Twente) and dr. M. Haalboom (clinical epidemiologist, Medisch Spectrum Twente/ postdoctoral researcher, University of Twente) for their support with the statistical analyses. The authors also thank S. Cnossen, MSc., for his contribution during his Technical Medicine internship.

\section{Declaration of conflicting interests}

R.H. Geelkerken is consultant for Terumo Aortic.

\section{Funding}

This study was funded in part by an unrestricted research grant from Vascutek Terumo, trading as Terumo Aortic, and in part by the PPP Allowance made available by Health Holland, Top Sector Life Sciences \& Health, to stimulate public-private partnerships.

\section{References}

1. Prinssen M, Verhoeven ELG, Buth J, Cuypers PWM, van Sambeek MRHM, Balm R, et al. A Randomized Trial Comparing Conventional and Endovascular Repair of Abdominal Aortic Aneurysms. N Engl J Med. 2004;351:1607-18.

2. Patel R, Sweeting MJ, Powell JT, Greenhalgh RM. Endovascular versus open repair of abdominal aortic aneurysm in 15-years' follow-up of the UK endovascular aneurysm repair trial 1 (EVAR trial 1): a randomised controlled trial. Lancet. 2016;388:2366-74.

3. Nordon IM, Karthikesalingam A, Hinchliffe RJ, Holt PJ, Loftus IM, Thompson MM. Secondary Interventions Following Endovascular Aneurysm Repair ( EVAR ) and the Enduring Value of Graft Surveillance. Eur J Vasc Endovasc Surg. 2010;39:547-54.

4. Jonker LT, de Niet A, Reijnen MMPJ, Tielliu IFJ, Zeebregts CJ. Mid- and Long-Term Outcome of Currently Available Endografts for the Treatment of Infrarenal Abdominal Aortic Aneurysm. Surg Technol Int. 2018;33:239-50.

5. Rödel SGJ, Zeebregts CJ, Huisman AB, Geelkerken RH. Results of the Anaconda endovascular graft in abdominal aortic aneurysm with a severe angulated infrarenal neck. J Vasc Surg. 2014;59:1495-1501.e1.

6. Freyrie A, Gallitto E, Gargiulo M, Faggioli G, Massoni CB, Mascoli C, et al. Results of the endovascular abdominal aortic aneurysm repair using the Anaconda aortic endograft. J Vasc Surg. 2014;60:1132-9.

7. Rödel SGJJ, Zeebregts CJ, Meerwaldt R, Van der Palen J, Geelkerken RH, Palen J Van Der, et al. Incidence and treatment of limb occlusion of the Anaconda endograft after endovascular aneurysm repair. J Endovasc Ther. 2018;26:113-20. 
8. Hammond A, Hansrani V, Lowe C, Asghar I, Antoniou SA, Antoniou GA. Meta-analysis and meta-regression analysis of iliac limb occlusion after endovascular aneurysm repair. J Vasc Surg. 2018;68:1916-1924.e7.

9. Faure EM, Becquemin JP, Cochennec F. Predictive factors for limb occlusions after endovascular aneurysm repair. J Vasc Surg. 2015;61:1138-45.

10. Mantas GK, Antonopoulos CN, Sfyroeras GS, Moulakakis KG, Kakisis JD, Mylonas SN, et al. Factors predisposing to endograft limb occlusion after endovascular aortic repair. Eur J Vasc Endovasc Surg. 2015;49:39-44.

11. Georgakarakos E, Argyriou C, Schoretsanitis N, Ioannou C V., Kontopodis N, Morgan R, et al. Geometrical Factors Influencing the Hemodynamic Behavior of the AAA Stent Grafts: Essentials for the Clinician. Cardiovasc Intervent Radiol. 2014;37:1420-9.

12. Carroccio A, Faries PL, Morrissey NJ, Teodorescu V, Burks JA, Gravereaux EC, et al. Predicting iliac limb occlusions after bifurcated aortic stent grafting: Anatomic and device-related causes. J Vasc Surg. 2002;36:679-84.

13. Maleux G, Koolen M, Heye S, Nevelsteen A. Limb Occlusion after Endovascular Repair of Abdominal Aortic Aneurysms with Supported Endografts. J Vasc Interv Radiol. 2008;19:1409-12.

14. Guan X, Guan X, Martonen TB, Martonen TB. Simulations of flow in curved tubes. Aerosol Sci Technol. 1997;26:485-504.

15. Koenrades MA, Klein A, Leferink AM, Slump CH, Geelkerken RH. Evolution of the Proximal Sealing Rings of the Anaconda Stent-Graft After Endovascular Aneurysm Repair. J Endovasc Ther. 2018;25:480-91.

16. Freyrie A, Gargiulo M, Rossi C, Losinno F, Testi G, Mauro R, et al. Preliminary Results of Anaconda $^{\text {TM }}$ Aortic Endografts: A Single Center Study. Eur J Vasc Endovasc Surg. 2007;34:693-8. 17. Rödel SGJ, Geelkerken RH, Prescott RJ, Florek HJ, Kasprzak P, Brunkwall J. The Anaconda ${ }^{\text {TM }}$ AAA Stent Graft System: 2-Year Clinical and Technical Results of a Multicentre Clinical Evaluation. Eur J Vasc Endovasc Surg. 2009;38:732-40.

18. Klein A, Jan Renema WK, der Vliet JA van, J.Oostveen L, Hoogeveen Y, Schultze Kool LJ, et al. Motion Calculations on Stent Grafts in AAA. In: Grundmann RT, editor. Diagnosis, Screen Treat Abdominal, Thoracoabdominal Thorac Aortic Aneurysms. [Rijeka]: InTechOpen; 2011. p. $125-44$.

19. Klein A, van der Vliet JA, Oostveen LJ, Hoogeveen Y, Kool LJS, Renema WKJ, et al. Automatic segmentation of the wire frame of stent grafts from CT data. Med Image Anal. 2012;16:127-39.

20. Koenrades MA, Struijs EM, Klein A, Kuipers H, Reijnen MMPJ, Slump CH, et al. Quantitative stent-graft motion in ECG-gated CT by image registration and segmentation: In vitro validation and preliminary clinical results. Eur J Vasc Endovasc Surg. 2019; [Epub ahead of print].

21. Schuurmann RCL, Kuster L, Slump CH, Vahl A, Van Den Heuvel DAF, Ouriel K, et al. Aortic curvature instead of angulation allows improved estimation of the true aorto-iliac trajectory. Eur J Vasc Endovasc Surg. 2016;51:216-24.

22. Harris P, Brennan J, Martin J, Gould D, Bakran A, Gilling-Smith G, et al. Longitudinal Aneurysm Shrinkage Following Endovascular Aortic Aneurysm Repair: A Source of Intermediate and Late Complications. J Endovasc Surg. 1999;6:11-6.

23. Di Achille P, Tellides G, Figueroa CA, Humphrey JD. A haemodynamic predictor of intraluminal thrombus formation in abdominal aortic aneurysms. Proc R Soc A Math Phys Eng Sci. 2014;470:20140163.

24. Chuter TAM. Durability of Endovascular Infrarenal Aneurysm Repair: When Does Late Failure Occur and Why? Semin Vasc Surg. 2009;22:102-10.

25. Lindblad B, Dias N, Malina M, Ivancev K, Resch T, Hansen F, et al. Pulsatile Wall Motion (PWM) measurements after endovascular abdominal aortic aneurysm exclusion are not useful in the classification of endoleak. Eur J Vasc Endovasc Surg. 2004;28:623-8.

26. Vos AWF, Wisselink W, Marcus JT, Vahl AC, Manoliu RA, Rauwerda JA. Cine MRI Assessment of Aortic Aneurysm Dynamics before and after Endovascular Repair. J Endovasc Ther. 2003;10:433-9. 
27. Demanget N, Duprey A, Badel P, Orgéas L, Avril S, Geindreau C, et al. Finite Element Analysis of the Mechanical Performances of 8 Marketed Aortic Stent-Grafts. J Endovasc Ther. 2013;20:523-35.

28. Itoga NK, Suh G-Y, Cheng CP. Stabilization of the Abdominal Aorta During the Cardiac Cycle with the Sac-Anchoring Nellix Device. Ann Vasc Surg. 2018;52:312.e7-312.e12.

29. Lee K, Hossain S, Sabalbal M, Dubois L, Duncan A, DeRose G, et al. Explaining endograft shortening during endovascular repair of abdominal aortic aneurysms in severe aortoiliac tortuosity. J Vasc Surg. 2017;65:1297-304.

30. Harris PL, Buth J, Mialhe C, Myhre HO, Norgren L. The Need for Clinical Trials of Endovascular Abdominal Aortic Aneurysm Stent-Graft Repair: The EUROSTAR Project. J Endovasc Ther. 1997;4:72-7. 
Appendix A 


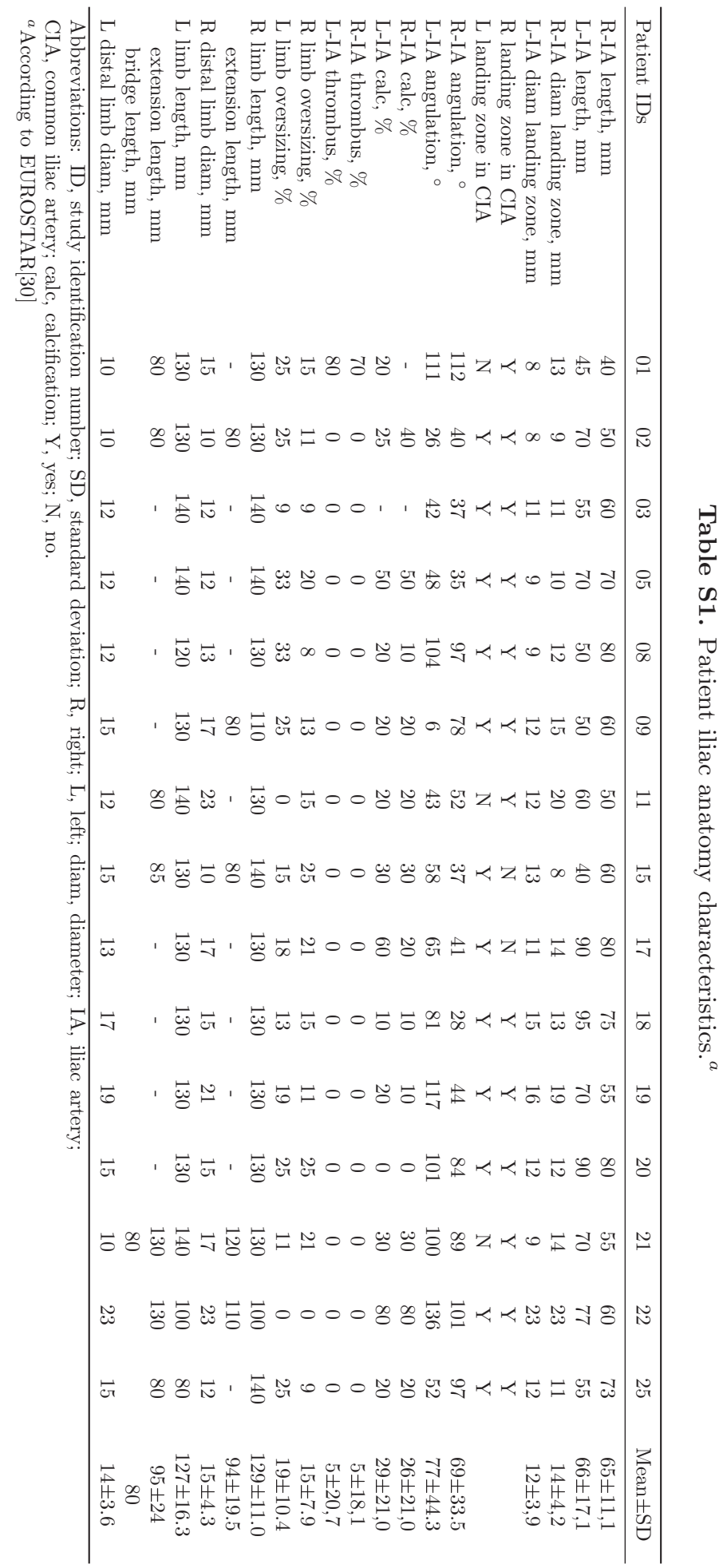




\section{Part II}

\section{Cardiac cycle related behavior}



pe

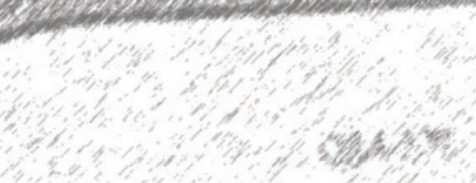

(lo

1.8

,

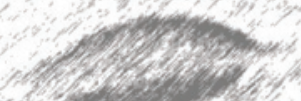

19.0

2010
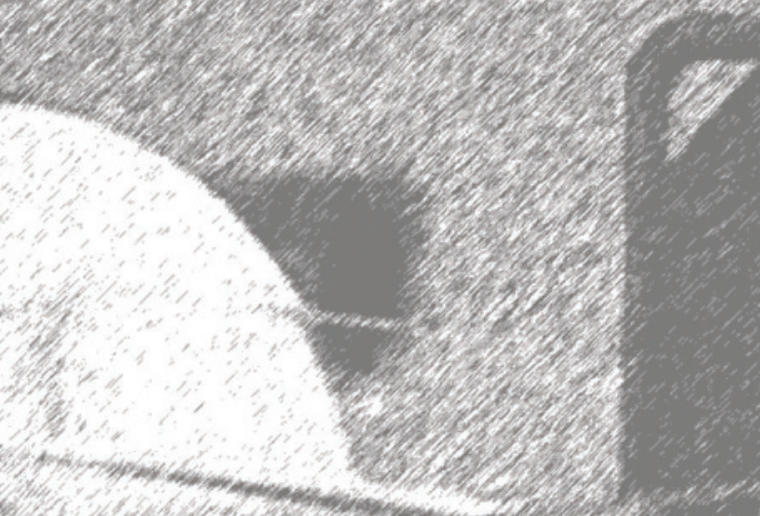

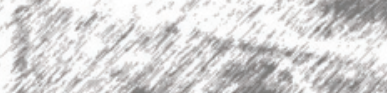

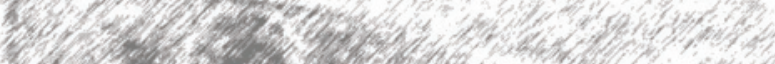

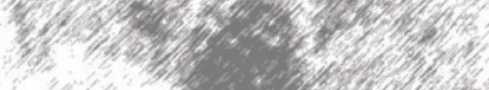

1.

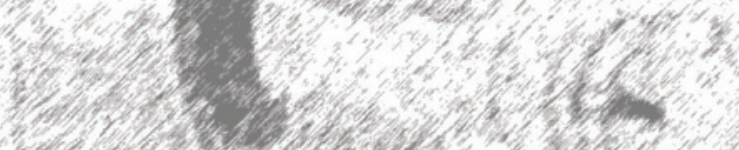

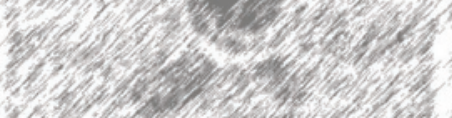

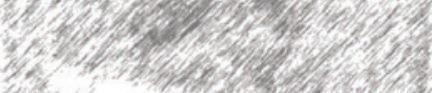

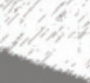

1.

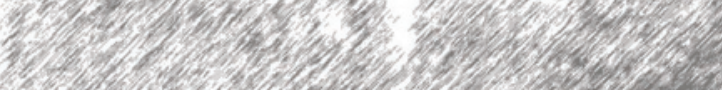

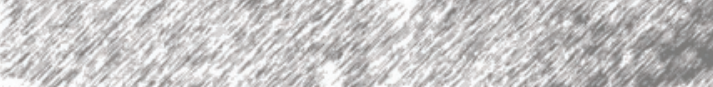

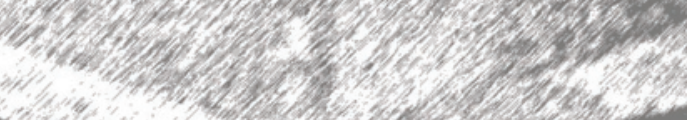

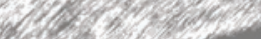

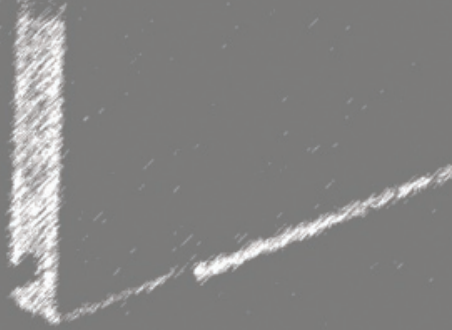





\section{Chapter 6}

\section{Quantitative stent-graft motion in ECG-gated CT by image registration and segmentation: In vitro validation and preliminary clinical results}

Maaike A. Koenrades ${ }^{a, b, c}$, Esmeralda M. Struijs ${ }^{d}$, Almar Klein $^{e}$, Hendrik Kuipers ${ }^{c}$, Michel M.P.J. Reijnen $^{a, f}$, Cornelis H. Slump ${ }^{c}$, Robert H. Geelkerken ${ }^{a, b}$

${ }^{a}$ Multi-modality Medical Imaging (M3I) group, Faculty of Science and Technology, Technical Medical Centre, University of Twente, Enschede, the Netherlands

${ }^{b}$ Department of Vascular Surgery, Medisch Spectrum Twente, Enschede, the Netherlands

${ }^{c}$ Robotics and Mechatronics (RaM) group, Faculty of Electrical Engineering, Mathematics and Computer Science, Technical Medical Centre, University of Twente, Enschede, the Netherlands

${ }^{d}$ Technical Medicine, Faculty of Science and Technology, Technical Medical Centre, University of Twente, Enschede, the Netherlands

${ }^{e}$ Independent Scholar, Enschede, the Netherlands

${ }^{f}$ Department of Vascular Surgery, Rijnstate, Arnhem, the Netherlands

Preliminary data from this study were presented at the SPIE Medical Imaging conference (February 11 - 16, 2017; Orlando, USA) 


\begin{abstract}
Objective: The dynamic endovascular environment of stent-grafts may influence long-term outcome after endovascular aneurysm repair (EVAR). The sealing and fixation of a stent-graft to the aortic wall is challenged at every heartbeat, yet knowledge on cardiac-induced dynamics of stent-grafts is sparse. Understanding the stent-artery interaction is crucial for device development and may aid the prediction of failure in the individual patient. The aim of this work was to establish quantitative stent-graft motion in multiphasic electrocardiogram (ECG)-gated computed tomography $(\mathrm{CT})$ by image registration and segmentation techniques.
\end{abstract}

Methods: Experimental validation was performed by evaluating a series of ECGgated CT scans of a stent-graft moving at different amplitudes of displacement at different virtual heart rates using a motion generating device with synchronized ECG-triggering. The methodology was further tested on clinical data of patients treated with EVAR devices with different stent-graft designs. Displacement during the cardiac cycle was analyzed for points on the fixating stent-rings, the branches or fenestrations, and the spine.

Results: Errors for the amplitude of displacement measured in vitro at individual points on the wire frame were at most $0.3 \mathrm{~mm}$. In situ cardiac-induced displacement of the devices was found to differ per location and also depended on the type of stent-graft. Displacement during the cardiac cycle was greatest in a fenestrated device and smallest in a chimney graft sac-anchoring endosystem, with maximum displacement varying from 0.0 to $1.4 \mathrm{~mm}$. There was no substantial displacement measurable in the spine.

Conclusion: A novel methodology to quantify and visualize stent-graft motion in multiphasic ECG-gated CT has been validated in vitro and tested in vivo. This methodology enables further exploration of in situ motion of different stent-grafts and branch stents and their interaction with native vessels.

Keywords: Stent graft motion, endovascular aneurysm repair, in vitro validation, image registration, dynamic quantification, ECG-gated computed tomography 


\section{Introduction}

Stent-grafts are implanted in a dynamic endovascular environment, which undoubtedly affects long-term patient outcome[1,2]. The long-term outcome of endovascular aneurysm repair (EVAR) is still a reason for concern[3-5], even though EVAR has become widely accepted for the treatment of aortic aneurysms. Every heartbeat, the fixation and sealing of the stent-graft to the aortic wall is challenged. If the sealing fails, blood flow can again enter the aneurysm sac resulting in type I endoleak and the risk of rupture returns. Additionally, device configurations are becoming increasingly complex, including the use of fenestrated (FEVAR) and branched stent-grafts (BEVAR), sac-anchoring endosystems (EVAS), and chimney grafts (CHEVAR/CHEVAS)[6], which may jeopardize the durability. Cardiac-induced motions may cause component dislocation or detachment of the stent-graft from the vessel wall, leading to migration, endoleak and/or kinking[1,2]. The forces on the stent may even change in time, due to changes in the stent expansion, in patients presenting with neck dilatation. Stent-graft migration and endoleak have shown to be responsible for reintervention within 6 years after endovascular treatment in $5-29 \%$ of the patients $[6-9]$. Also, stent fracture may occur due to continuous cyclic deformation that exerts mechanical stress on the wire frame, resulting in fatigue. Depending on the type of stent-graft and the location of the fracture, this may have serious consequences[10,11]. Conversion to resolve failing EVAR is accompanied with a substantial morbidity and mortality[12] and FEVAR in a failed EVAR device is complex and may lead to technical failure due to the inability to cannulate the side branches[13].

Failure of EVAR may occur years after treatment. Besides anatomical changes in time, including neck enlargement, stent-related factors could contribute to these late failures. Manufacturers use computational modeling and accelerated fatigue tests to optimize their designs. However, these techniques are mainly based on assumptions and often lack actual knowledge of in situ cardiac-induced motion. A realistic simulation of the endovascular environment, including patient-specific physiological and mechanical properties, is essential for accurate failure prediction[1]. Fatigue tests for example rely on the extent of stress and strain imposed on the wire frame of the stentgraft. The reliability of computational techniques that aim to provide insight in the distribution of stress and strain, such as finite element analysis, highly depends on the degree to which they can be validated against actual in situ measurements of strain. It is therefore highly relevant to obtain information on the motion of stent-grafts in situ, both to perform risk assessment in the preclinical stage during device development and for early prediction of failure in the individual patient in post-market evaluations of new or modified devices.

Detailed knowledge on the motions of implanted stent-grafts is limited, since routine follow-up imaging is commonly restricted to static computed tomography (CT) that does not allow for measurement of motion. Electrocardiogram (ECG)-gated (3D+time) CT can be used to quantify motions of stent-grafts[14,15] and the aortic vessel[2,16-23], although validation studies are scarce[24,25]. Most studies have evaluated pulsatile distention of the aorta during the cardiac cycle by measuring aortic dimensions perpendicular to a central luminal line in multiplanar reconstructions at predetermined levels[2,17-23]. A disadvantage of using 2-dimensional cross sectional 

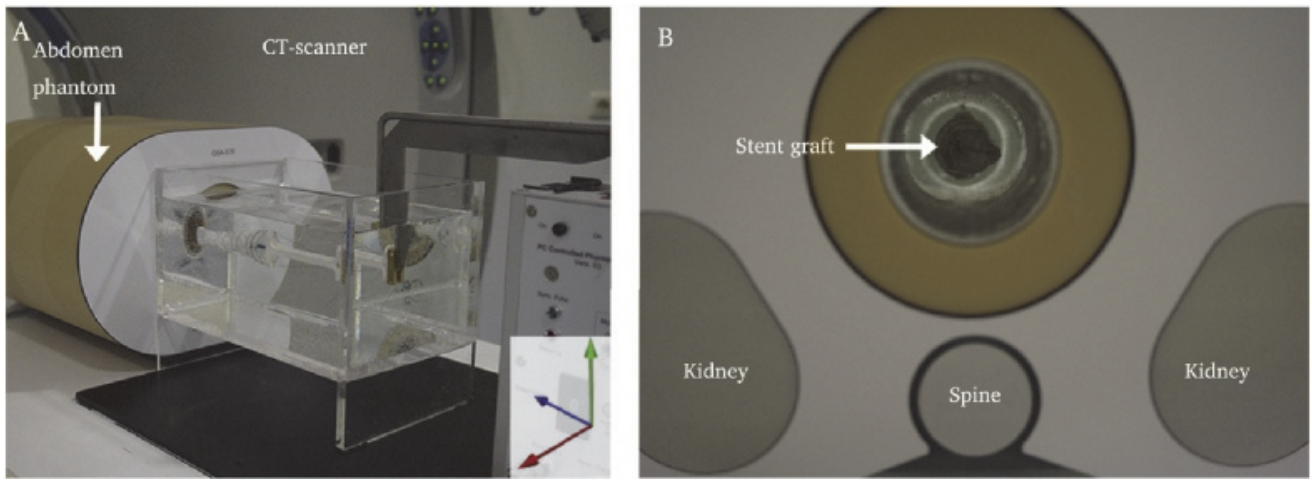

Figure 1. The experimental setup with CT scanner: the stent-graft was moving in $z$ direction (A) inside the aortic abdomen phantom (B). The axes of the coordinate system (A) denote the $x$-(red), $y$-(green) and $z$-direction (blue). CT, computed tomography.

images is that it does not allow for correction of movement out of plane in cranial or caudal direction and thus not allow for three dimensional measurement of motion. Also, in these studies measurements were performed separately in each of the reconstructed phases, which is prone to measurement inaccuracies, resulting in relatively high observer variability in the range of the measured motion[19,20]. Moreover, there was no reference for the actual in situ motion to validate the measurements.

The current study was conducted to provide accurate data characterizing the motion of implanted stent-grafts. The method used was based on a previously established combination of an image registration and a segmentation algorithm to quantitatively and qualitatively assess motions of stent-grafts and their interaction with native vessels in time-resolved CT data[15]. The method was validated in vitro and further tested on clinical data of patients treated with EVAR devices with different stentgraft designs.

\section{Methods}

\section{In vitro validation experiments}

Experimental setup A motion generating device with synchronized ECGtriggering (PC controlled phantom device, QRM Quality Assurance in Radiology and Medicine GmbH, Möhrendorf, Germany[26]) was used to obtain a series of ECGgated CT scans with a stent-graft moving according to predetermined patterns with different amplitudes of motion at different virtual heart rates. The main body of an Anaconda stent-graft (Terumo Aortic, Inchinnan, Scotland, UK) was attached to the lever of the motion device. The body of this stent-graft comprises two nitinol O shaped stent-rings to provide fixation and sealing of the stent-graft against the vessel wall. The lever was placed in a water filled polyacrylic container with cylindrical tube (diameter, $45 \mathrm{~mm}$; wall thickness, $2 \mathrm{~mm}$ ) to fit the hole of an anthropomorphic aortic abdomen phantom (QRM GmbH; dimensions $x y z, 300 \times 200 \times 250 \mathrm{~mm}^{3}$; hole diameter, 
$45 \mathrm{~mm}$ ). This phantom was developed to mimic a cross section of the human abdomen for CT imaging, comprising of spine, kidneys and soft tissue equivalent material. The experimental setup is shown in Figure 1.

Abdominal aortic motion patterns A motion pattern composed of Gaussian functions was designed to mimic aortic motion. By providing the pattern's frequency and amplitude two series of motion patterns were generated. In the A series the heart rate was changed while the amplitude remained constant and in the B series vice versa. Note that with amplitude we refer to the maximum absolute value of displacement, that is, the maximum distance between two positions during a full cycle. Two motion patterns in the B series were given a second peak to simulate irregular aortic motion, resembling non-uniform patterns[27] and wave reflection patterns[28]. The amplitudes chosen were relatively small since the stent-graft is expected to locally decrease the motion of the abdominal aorta, which has been reported to be in the order of 1-2 $\mathrm{mm}[16,27]$. Detailed information on the generated motion patterns is available in Supplemental Resource 1.

Validation of the motion device To obtain a sub-mm accurate ground truth for algorithm validation, a high resolution camera was used to assess the patterns executed by the motion device. Details of this in vitro validation and the acquired reference patterns are available in Supplemental Resource 1 and corresponding Video S1 and S2.

ECG-gated CT scans Experiments were performed on two CT scanners that were recently used to acquire ECG-gated CT scans in a clinical trial (Trialregister.nl identifier NTR4276): an Aquilion 64 CT scanner (Toshiba Medical Systems Corporation, Tokyo, Japan) and a Somatom Definition Flash CT scanner (Siemens Healthineers, Forchheim, Germany). The motion device was scanned with a helical scan protocol while executing the generated motion patterns. Time-resolved ECG-gated scans were obtained by triggering the CT scanners with the motion device. Scan and reconstruction parameters were in accordance with the clinical trial and were similar for both scanners: rotation time, 0.4 seconds (Aquilion), 0.285 seconds (Flash); collimation, $64 \times 0.5 \mathrm{~mm}$ (Aquilion), $2 \times 128 \times 0.6 \mathrm{~mm}$ (Flash); slice thickness, $1 \mathrm{~mm}$; slice overlap (increment), $0.5 \mathrm{~mm}$; tube voltage, $120 \mathrm{kV}$; tube current time product, $60 \mathrm{mAs}$. Data were reconstructed with a FC12 (Aquilion) and I36f (Flash) convolution kernel, a matrix size of $512 \times 512$ pixels, and a field of view of approximately $250 \times 250 \mathrm{~mm}^{2}$, resulting in approximately isotropic voxels of $0.5 \mathrm{~mm}^{3}$. The pitch factor was set automatically based on the heart rate by which the duration of the scans varied between 10 and 20 seconds. Retrospective gating was applied to obtain 10 equidistant volumes covering the cardiac cycle (i.e., $0-90 \%$ of the RR-interval). The resulting data were cropped to $256 \times 256 \times 200$ voxels $(x y z)$ to reduce memory requirements.

Motion modeling by image registration and segmentation Motion of the stent-graft was quantitatively and qualitatively assessed by applying a combined image registration and segmentation algorithm[15,29,30]. This method involved three 
A

Groupwise image registration
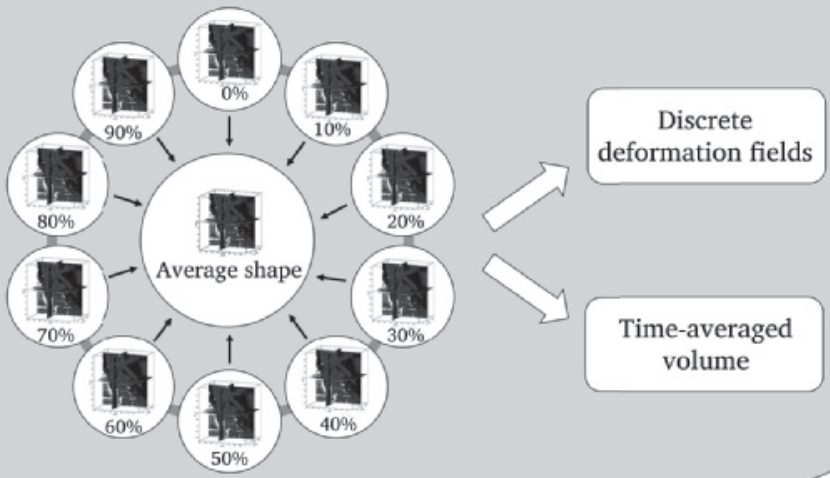

B

Image segmentation
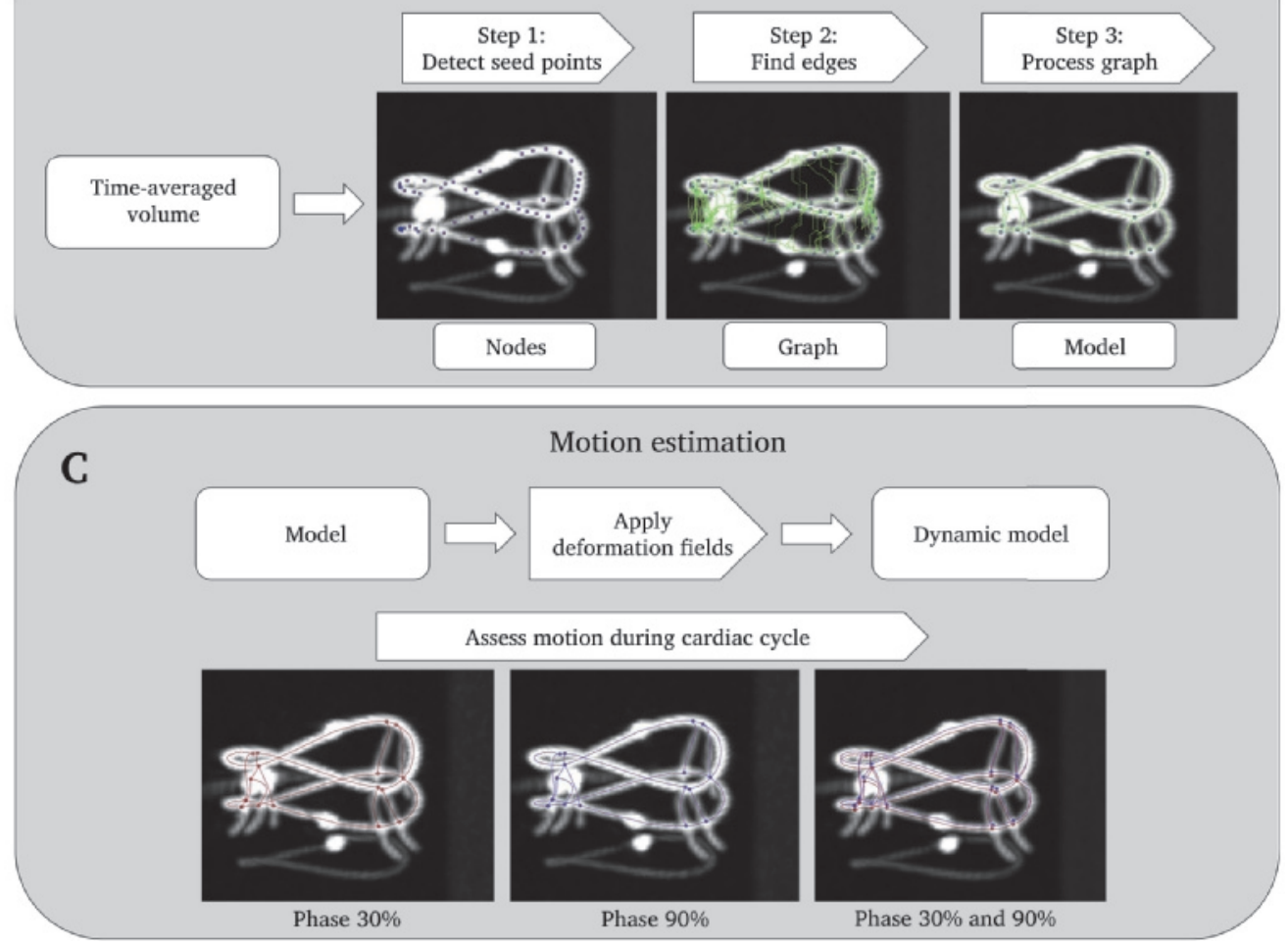

Figure 2. Illustration of the image processing steps, including (A) groupwise image registration by simultaneously aligning all time-resolved 3D CT volumes towards their 'mean shape'; (B) segmentation of the stent-rings in the time-averaged volume; and (C) motion estimation during the cardiac cycle by applying the deformation fields to the stent-ring model to estimate the position of the stent-rings at different time points in the cardiac cycle. As an example, a model is shown at $30 \%$ and $90 \%$ of the cardiac cycle. The combined view shows the position of the model at both time points with respect to the time-averaged volume. 3D, three dimensional. 
Table 1. Experimental results of the pattern amplitude errors obtained for two CT scanners.

\begin{tabular}{llllll}
\hline P & $\begin{array}{l}\text { A } \\
(\mathrm{mm})\end{array}$ & \multicolumn{2}{c}{ Aquilion 64 scanner } & \multicolumn{2}{c}{ Somatom Definition Flash scanner } \\
& & Error $(\mathrm{mm})$ & Error $(\%)$ & Error $(\mathrm{mm})$ & Error $(\%)$ \\
\hline A1 & 0.72 & $0.08 \pm 0.05(0.03-0.17)$ & $12 \pm 6(4-24)$ & $0.06 \pm 0.05(0-0.13)$ & $8 \pm 6(0-18)$ \\
A2 & 0.75 & $0.07 \pm 0.04(0-0.15)$ & $9 \pm 6(1-20)$ & $0.09 \pm 0.08(0.02-0.24)$ & $12 \pm 10(3-32)$ \\
A3 & 0.70 & $0.11 \pm 0.05(0.03-0.16)$ & $16 \pm 7(5-23)$ & $0.16 \pm 0.06(0.07-0.25)$ & $23 \pm 9(9-36)$ \\
B0 & 0 & $0.03 \pm 0.03(0-0.08)$ & - & $0.21 \pm 0.03(0.17-0.26)$ & - \\
B1 & 0.23 & $0.10 \pm 0.05(0.01-0.17)$ & $43 \pm 23(6-73)$ & $0.06 \pm 0.04(0.01-0.14)$ & $27 \pm 19(5-58)$ \\
B2 & 0.37 & $0.06 \pm 0.04(0.02-0.12)$ & $15 \pm 10(5-32)$ & $0.16 \pm 0.04(0.09-0.21)$ & $42 \pm 10(25-56)$ \\
B3 & 0.70 & $0.06 \pm 0.03(0-0.10)$ & $8 \pm 5(2-15)$ & $0.11 \pm 0.08(0.01-0.23)$ & $16 \pm 11(1-32)$ \\
B4 & 1.38 & $0.06 \pm 0.09(0-0.27)$ & $5 \pm 7(0-20)$ & $0.07 \pm 0.06(0-0.16)$ & $5 \pm 4(0-12)$ \\
B5 & 1.26 & $0.07 \pm 0.07(0-0.24)$ & $6 \pm 6(0-19)$ & $0.10 \pm 0.09(0.03-0.29)$ & $8 \pm 7(2-23)$ \\
B6 & 1.24 & $0.09 \pm 0.08(0-0.23)$ & $7 \pm 6(1-19)$ & $0.13 \pm 0.09(0.01-0.30)$ & $10 \pm 7(1-24)$ \\
\hline
\end{tabular}

Abbreviations: CT, computed tomography; P, motion pattern; A, amplitude of reference pattern. Data are presented as mean \pm standard deviation (range). Values are given in millimeters and as a percentage of A. A3 and B3 are repeated measures and have the same reference.

steps: 1) deformable (i.e., elastic) registration of the time-resolved 3D volumes to acquire deformation (i.e., vector) fields that describe the displacement of all voxels in all phases with respect to the average of all phases (hereafter referred to as time-averaged volume); 2) segmentation of the stent-rings, resulting in a geometric model that represents the wire frame and consists of nodes at wire crossings and edges connecting the nodes; and 3) applying the deformation fields to the geometric model to assess motion of the model throughout the phases. This process is illustrated in Figure 2. Detailed information is provided in Supplemental Resource 2. The algorithms were coded in Python programming language (version 3).

Algorithm validation Displacement patterns of the models as estimated by the algorithm were compared with the motion device reference patterns. The node points ( 8 per ring) and the points halfway along the edges of the rings ( 8 per ring) were used, resulting in 32 points per model. Errors were calculated by subtracting the values of maximum displacement, i.e., pattern amplitudes errors, and by subtracting the respective patterns over the full cardiac cycle by taking the reference pattern positions at the correct times, that is, the positions at 10 equidistant time-intervals corresponding to the 10 volumetric phases of the gated CT scans.

Statistics Data were normally distributed and summarized as mean \pm standard deviation and range, and were compared between the two respective CT scanners using a dependent Student's t-test for paired samples. A $p$ value $<0.05$ was considered significant. Statistical analysis was performed using the open source statistical functions of Scipy 1.1 with Miniconda3 as Python interpreter (Python 3).

Clinical application The clinical applicability of the algorithm was evaluated using postoperative ECG-gated CT scans of two EVAR cases (Anaconda device, 

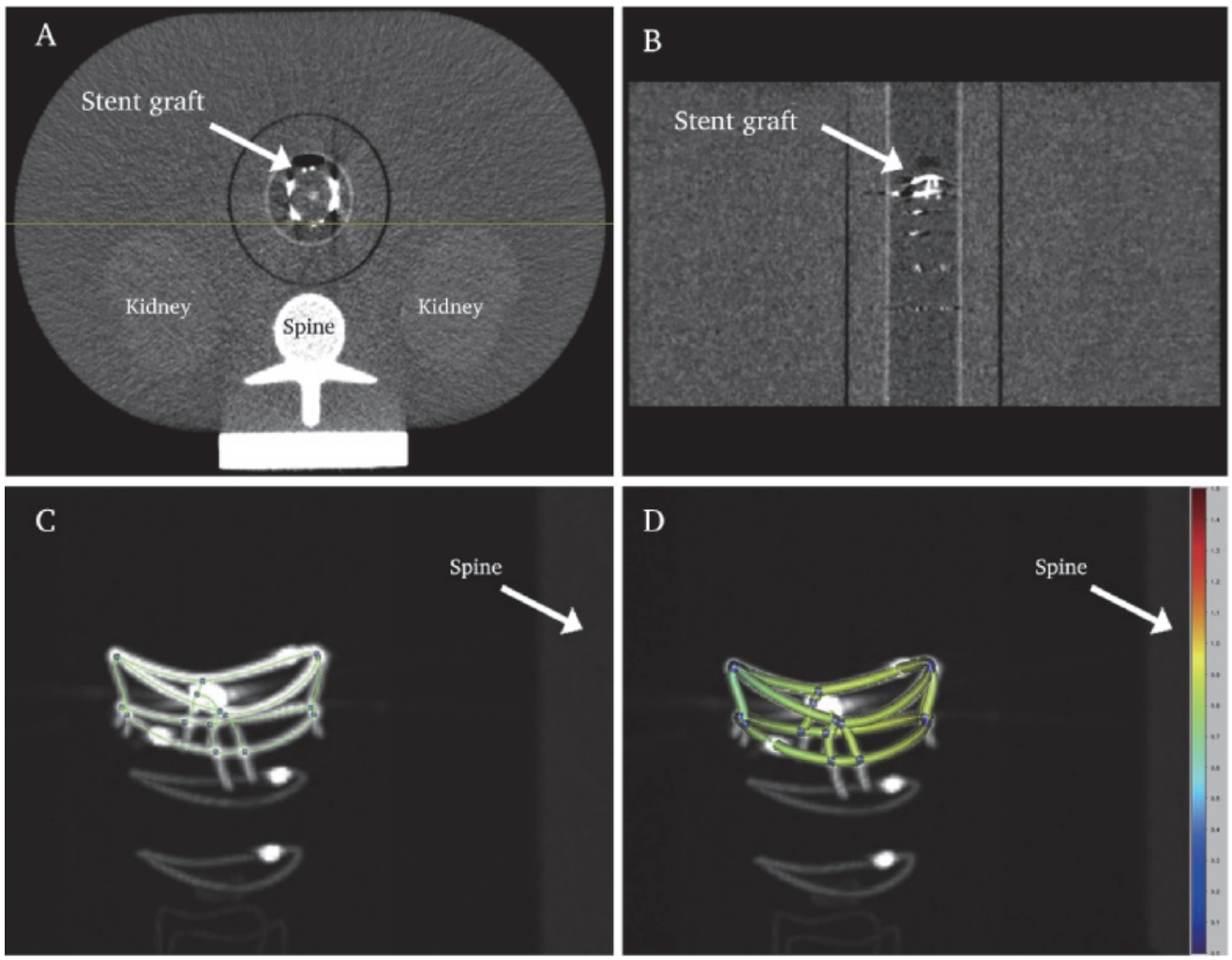

Figure 3. Example of a dynamic model that was acquired from the Aquilion CT data (motion pattern A3), showing an (A) axial and (B) coronal multiplanar reconstruction (MPR) slice of the time averaged volume and $(\mathrm{C}, \mathrm{D})$ three dimensional maximum intensity projections (MIP's) with the geometric model of the two upper rings for which the amplitude of motion is visualized in a color coded mesh on a scale from 0 to $1.5 \mathrm{~mm}$ (D, Video S3). The yellow horizontal line in the axial MPR slice (A) indicates the level of the coronal slice (B). The green lines and blue dots (C,D) represent the model edges and node points, respectively.

Terumo Aortic, Inchinnan, Scotland, UK; Endurant device, Medtronic, Santa Rosa, CA, USA), a FEVAR case (Fenestrated Anaconda device, Terumo Aortic) employing Advanta V12 covered stents (Maquet Getinge Group, Hudson, NH, USA), and a CHEVAS case (Nellix device, Endologix, Irvine, CA, USA) also employed Advanta V12 covered stents. The EVAR and FEVAR data were collected from clinical trial databases (Trialregister.nl identifier NTR4276 and NTR6225, respectively). The CHEVAS case was retrospectively retrieved from the ASCEND registry[31]. The data collection was approved by local ethical committees. The ECG-gated CT scans comprised 10 time-resolved CT volumes at 10\% steps of the cardiac cycle. Threedimensional displacement patterns were assessed for distinctive points on the proximal stent-rings and the branches or fenestrations by applying the image registration and segmentation algorithm. For validation purposes, the displacement of points in the spine was assessed. 


\section{Results}

\section{In vitro validation}

The groupwise registration process took approximately 12 minutes per scan on an Intel Core i7-5500U processor with $2.9 \mathrm{GHz}$ clock speed and 16 GB RAM. An example of a scan and obtained dynamic stent-ring model is shown in Figure 3. Figure 4 compares reference patterns with algorithm patterns for the CT data that was obtained on each scanner. Table 1 presents the absolute values of the amplitude errors. Mean amplitude errors were smaller than $0.11 \mathrm{~mm}$ and $0.21 \mathrm{~mm}$ for the image data acquired on the Aquilion and Flash scanner respectively. The maximum amplitude error for a single point in the model was $0.27 \mathrm{~mm}$ (B4) for the Aquilion data and $0.30 \mathrm{~mm}$ (B6) for the Flash data. Small but statistically significant differences were noted between amplitude errors for the Aquilion and Flash data for the patterns A1 ( $p=.045)$, A3 $(\mathrm{p}=.040), \mathrm{B} 0(\mathrm{p}<.001), \mathrm{B} 1(\mathrm{p}<.001), \mathrm{B} 2(\mathrm{p}=.004)$, and B3 $(\mathrm{p}=.003)$. Absolute errors over the full cycle (all 10 positions/ phases) are presented in Figure 5. The difference between errors for the Aquilion and Flash data was small but statistically significant for the patterns A1 (p<.001), A2 (p=.040), B0 ( $\mathrm{p}<.001), \mathrm{B} 1(\mathrm{p}<.001), \mathrm{B} 2(\mathrm{p}<.001)$, B4 $(p=.006)$, and B6 $(p=.020)$. Overall, mean absolute errors were smaller than 0.14 $\mathrm{mm}$ and $0.12 \mathrm{~mm}$ for the Aquilion and Flash data respectively.

Clinical application Figure 6 presents cardiac-induced displacement patterns in clinical ECG-gated CT data of an Anaconda (Figure 6A), an Endurant (Figure 6B), a Fenestrated Anaconda (Figure 6C), and a Chimney Nellix (Figure 6D) case. The amplitude of displacement over the cardiac cycle was greatest in the fenestrated case and smallest in the Nellix case, varying from 0.0 to $1.4 \mathrm{~mm}$. As to be expected, the peaks in the stent-graft motion patterns were found during the systolic phases of the cardiac cycle for the abdominal aorta. In all cases, the amplitude of displacement of the stent-graft was above the uttermost error threshold of $0.3 \mathrm{~mm}$, except for the Nellix case where only the branch showed moderate displacement. There was no substantial displacement measurable in the spine ( $x$-direction: $0.19 \pm 0.08 \mathrm{~mm}$; y-direction: $0.19 \pm 0.10$; $z$-direction: $0.18 \pm 0.08)$.

\section{Discussion}

This study validates a novel methodology combining image registration and segmentation techniques to quantify stent-graft motions on multiphasic ECG-gated CT. Since an absolute ground truth is lacking in clinical data, we have used a physical phantom to evaluate the accuracy of the methodology. This in vitro validation demonstrated that the error for the amplitude of motion at individual points on the stent-rings was small and at most $0.3 \mathrm{~mm}$, which is about half the pertinent voxel size. The clinical applicability of the algorithm was demonstrated for four different stent-graft designs in EVAR, FEVAR and CHEVAS cases. Because the aortic wall compliance generally increases from the abdominal to the thoracic region[32], it may be expected that the motion of the fixating stent-rings differs per location. This was indeed observed in this study, where the relative displacement, caused by the cardiac cycle, varied between 0.0 and $0.7 \mathrm{~mm}$. The maximum displacement also depended on the type of 

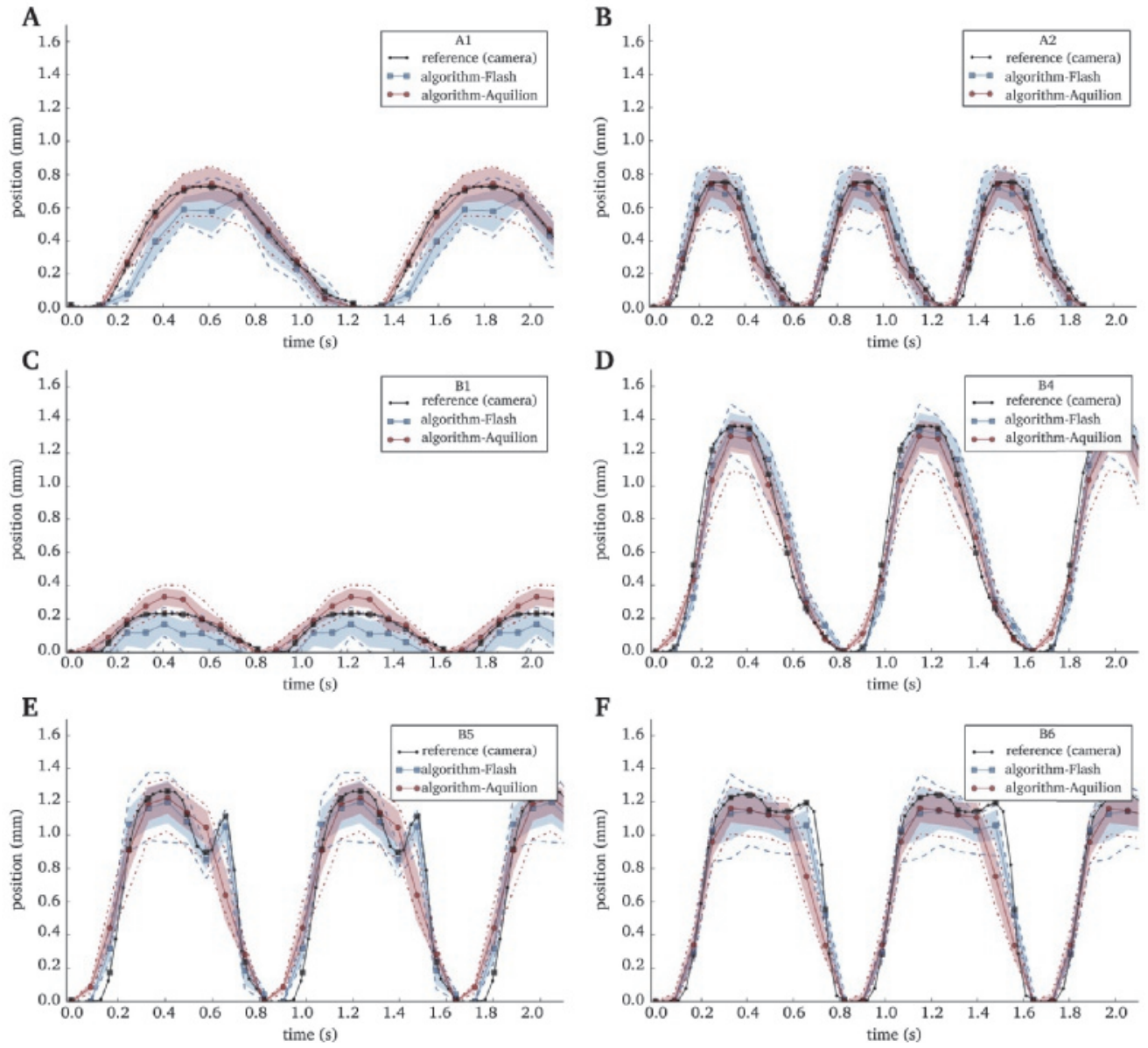

Figure 4. Reference versus algorithm for both the Aquilion and the Flash CT scanner for six motion patterns (A-F). Mean \pm standard deviation of the algorithm patterns is shown as well as the patterns showing the minimum and maximum displacement (dashed/dotted lines). The black squares in the reference patterns correspond to each of the 10 reconstructed phases in the cardiac cycle to compare algorithm and reference. 

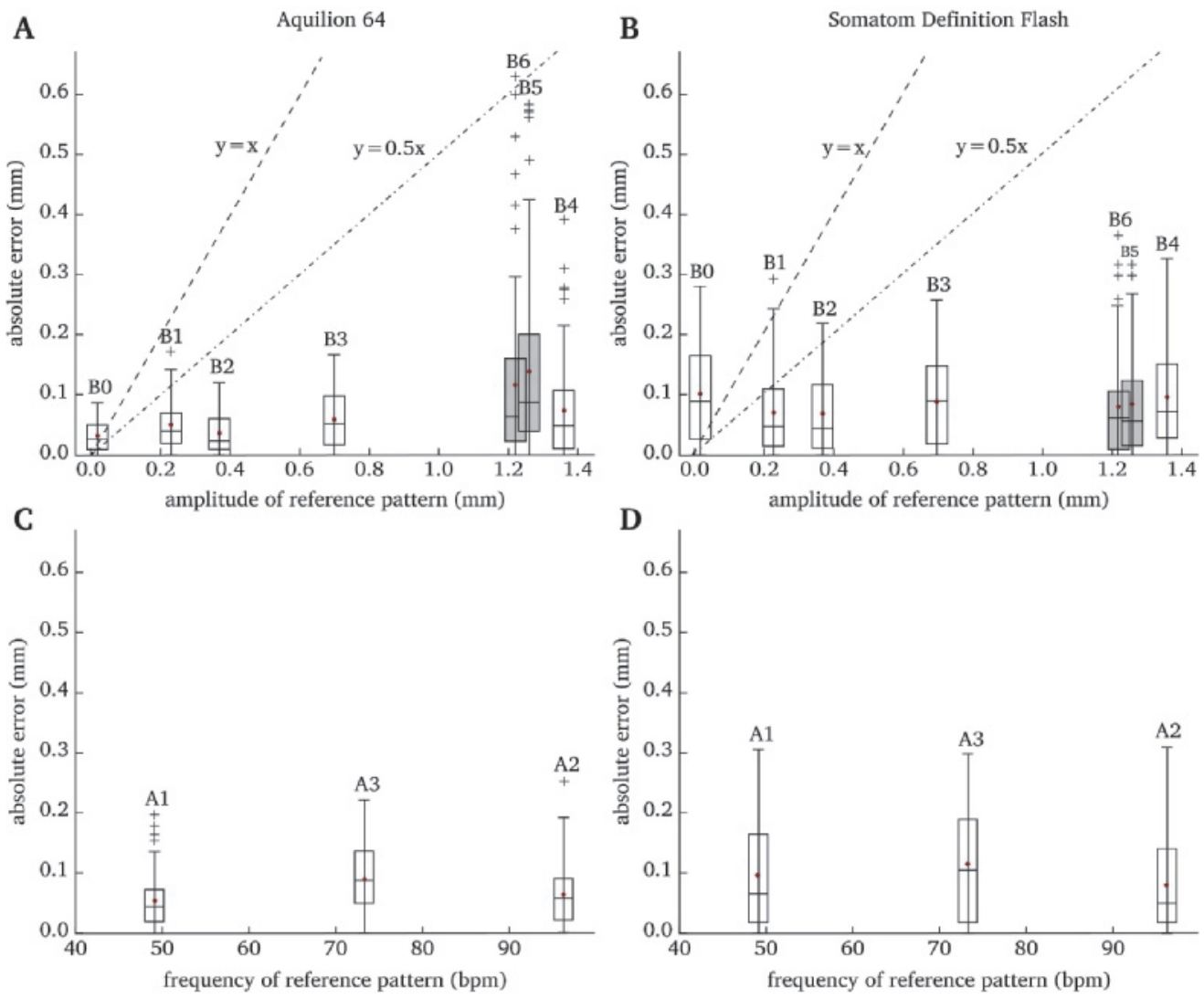

Figure 5. Absolute errors of the reference versus the algorithm over the full cycle for all 10 positions (10 phases) in the displacement patterns (Figure 4). Errors are shown for both the Aquilion (A,C) and the Flash (B,D) CT image data for the patterns with varying amplitude $(\mathrm{A}, \mathrm{B})$ and frequency $(\mathrm{C}, \mathrm{D})$. The red dots represent mean absolute errors. The whiskers represent 1.5 times the interquartile range. The two patterns with an extra peak (B5, B6) are shown with gray boxes. Reference lines at $y=x$ and $y=0.5 x$ are shown $(\mathrm{A}, \mathrm{B})$ to relate the error to the amplitude of motion. A3 and B3 are repeated measures and have the same reference. 
stent-graft. It is also not surprising that there was virtually no movement of the Nellix device, which uses rigid polymer-filled endobags to fill the aneurysmal lumen and stabilize two balloon-expandable covered stents in the aorta and iliac arteries. Yet, the dynamic behavior may change over time and can potentially be an early indicator of failure. More knowledge and understanding of this new endovascular treatment technique seems requisite, since the longer-term outcomes appear to be significantly inferior to other available treatment options[5].

While several studies have investigated the dynamic behavior of the aorta $[2,16$ 23], including a recent study that employed deformable registration techniques for the aortic arch[33], little has been reported on the dynamic behavior of stent-grafts. From a clinical point of view this information, however, is of utmost importance. Late failures after EVAR are not uncommon and necessitate prolonged follow-up. Early identification of those at risk for failure, may lead to a more individualized follow-up scheme and reduce the burden of prolonged follow-up. Langs et al. have proposed a method to study motion of stent-grafts in the thoracic aorta by applying a statistical shape variation model that is built during registration from a finite set of interest points, i.e., landmarks on the wire frame[14]. Using in situ patient data they evaluated correspondence quality of the registration by computing distances between original interest points in an individual phase of the cardiac cycle and interest points that were mapped according to the deformation field. They found mean registration errors between 0.25 and 0.73 voxels (in-plane xy dimensions, $0.65 \times 0.65 \mathrm{~mm}^{2}$ to $0.98 \times 0.98$ $\mathrm{mm}^{2}$; slice thickness, $0.625 \mathrm{~mm}$ ) but did not report the errors of individual interest points. These registration errors appear to be higher than the errors reported in the present work, which may be explained by the fact that the statistical models are based on a set of landmarks rather than a texture-based deformation of the entire volume as applied by the present algorithm.

In the in vitro validation experiments we compared the results of two CT scanners. Several patterns showed small yet statistically significant differences between the scanners. Several factors may have contributed to such differences, including the hardware of the scanners, the reconstruction process (e.g., interpolation, filtering), and the temporal resolution (Aquilion $200 \mathrm{~ms}$ vs. Flash $75 \mathrm{~ms}$ ). Notably, the extra peaks that were added in two patterns were not identified in the data of the Aquilion scanner, but these were identified in the data of the Flash scanner (Figure 4E,F). Overall, there was no consistent under- or overestimation of motions when comparing the errors of both scanners, which suggests that results would not require correction when comparing clinical data from the respective scanners.

A limitation of this work is that the experimental validation was performed in $z$ direction only. Nevertheless, three dimensional displacement patterns were analyzed in clinical ECG gated CT data, demonstrating that pulsatile motions can be measured in all three directions and may differ between stent-grafts. Additionally, there was no substantial motion in the spine, which suggests that the measured in vivo stent-graft motion was cardiac induced and not due to image artifacts or registration errors. Moreover, it was expected that the performance in $x$ - and $y$-direction is equal or even better compared to the performance in $z$-direction since, although the voxel dimensions were approximately the same in all dimensions $(0.5 \mathrm{~mm})$, the effective spatial resolution was higher in the $x-y$ dimensions because the slice thickness was 
A

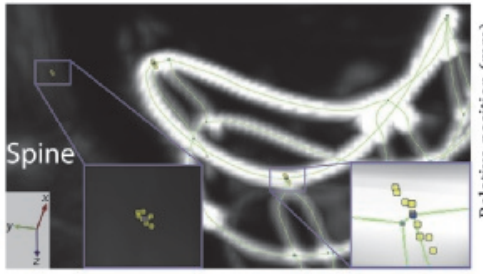

B

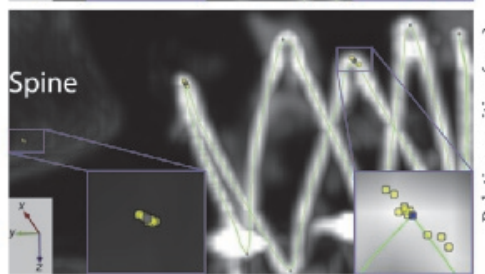

C

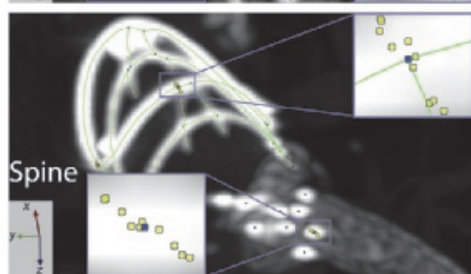

D

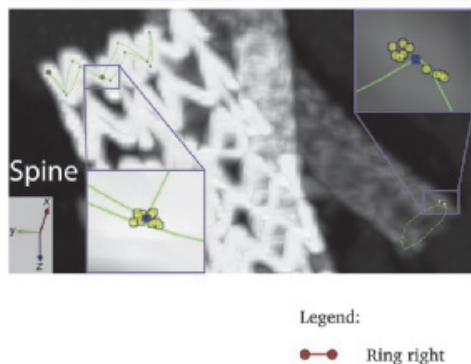

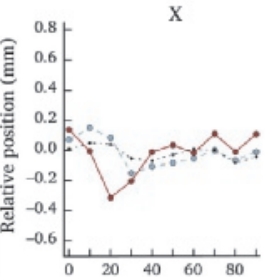
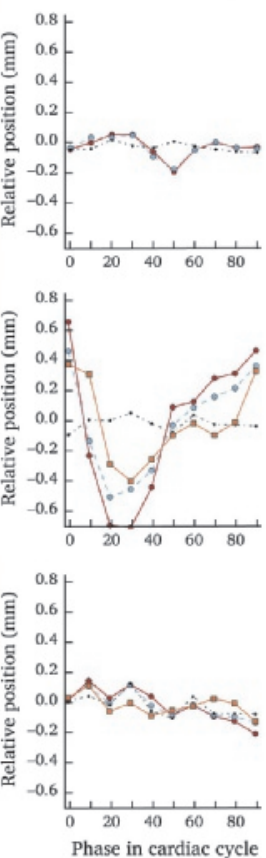

Phase in cardiac cycle

o - Ring posterior
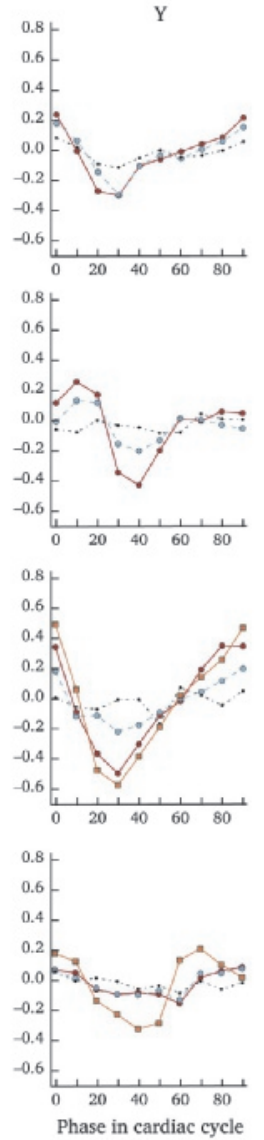

Phase in cardiac cycle
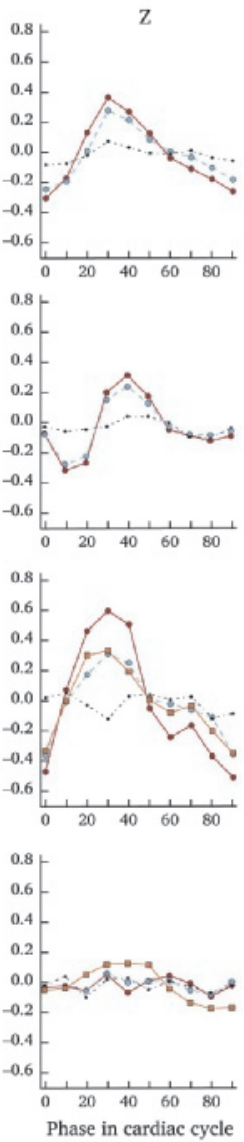

Figure 6. An in situ analysis of displacement during the cardiac cycle in postoperative ECGgated CT data of four abdominal aortic aneurysm patients who were treated endovascularly using an Anaconda (A), an Endurant (B), a Fenestrated Anaconda (C), or a Chimney Nellix (D) device configuration. Displacement is shown for individual points of the proximal stentrings, the fenestration or branch, and the spine. The three dimensional images display the position of the geometric models (model lines and blue dots) at mid-cardiac cycle in the time-averaged volume and the position of distinct, individual points at each phase in the cardiac cycle (yellow dots) with higher magnifications in the boxes. For each case the graphs display the relative position of the individual points with respect to the time-averaged volume as a function of the cardiac phase (in percent of RR-interval) in $x$-, $y$ - and $z$-direction, corresponding to the right $(-)$ to left $(+)$, anterior $(-)$ to posterior $(+)$, and cranial $(-)$ to caudal $(+)$ direction. The axes of the coordinate systems denote the $x$-(red), $y$-(green) and $z$-direction (blue). 
$1 \mathrm{~mm}$. Also, in previous work it was found that the detectability of motions was better in $x$-direction than in $z$-direction[25]. Furthermore, even though we mimicked pulsatile displacement profiles, the motion was simplified compared to the situation in vivo where the blood flow may also deform the stent-graft and the aorta (e.g., radial expansion), which may be more difficult to measure. Nonetheless, the employed deformable registration allows to measure such complex transformations[30]. Additional experiments may be performed with more realistic motion patterns by for example using synthetic data with known deformation fields or real-world patient data with extracted landmark trajectories, since it is virtually unfeasible to validate subtle deformations in physical experimental models.

This study provides evidence that the proposed algorithm is able to accurately measure (subvoxel) motions of aortic stent-grafts in ECG-gated CT. This enables to measure subtle cardiac-induced adaptations of the stent-graft in the aortic neck and therewith to evaluate the consequences of stent-grafting on aortic neck compliance. Moreover, such measurements allow for adequate preclinical tests and early clinical assessment of stent-graft efficacy and durability, including risk assessment for endoleak, migration, stent dislocation, and/or fracture.

\section{Conclusion}

Using a motion generating device, this study has demonstrated that the accuracy of the proposed registration and segmentation technique is adequate for measurement of abdominal aortic stent-graft motions on ECG-gated CT data. Errors for the amplitude of motion at individual points on the stent-rings were no more than $0.3 \mathrm{~mm}$, which supports the feasibility of measuring subtle motions in vivo. The algorithm was successfully applied to clinical ECG-gated CT data to measure and visualize three dimensional motions during the cardiac cycle in EVAR, FEVAR, and CHEVAS cases with different $\mathrm{Z}$ and $\mathrm{O}$ shaped stent-graft designs. This novel methodology offers the prospect to investigate in situ behavior of different stent-grafts and branch stents in the dynamic endovascular environment of the thoraco-abdominal aorta and branches.

\section{Acknowledgments}

The authors thank the Department of Radiology of the Medisch Spectrum Twente and Bert Klein Rot (Chief laboratory technician CT) in particular for providing technical support with the CT acquisitions. Furthermore, the authors thank the Department of Radiology of the University Medical Center Groningen (UMCG) and Prof.dr. Marcel J.W. Greuter (Head of Medical Physics UMCG) for providing us their QRM motion device to test the setup. Finally, the authors thank Prof.dr.ir. Bernard J. Geurts (Chairholder for Multiscale Modeling and Simulation in the Department of Applied Mathematics at the University of Twente) for critically reviewing the article.

\section{Declaration of conflicting interests}

The authors declare that there are no conflicts of interest. 


\section{Funding}

This study was funded in part by an unrestricted research grant from Vascutek Terumo, trading as Terumo Aortic, and in part by the PPP Allowance made available by Health Holland, Top Sector Life Sciences \& Health, to stimulate public-private partnerships.

\section{References}

1. Chuter TAM. Durability of Endovascular Infrarenal Aneurysm Repair: When Does Late Failure Occur and Why? Semin Vasc Surg. 2009;22:102-10.

2. Van Keulen JW, Moll FL, Barwegen GK, Vonken EPA, Van Herwaarden JA. Pulsatile distension of the proximal aneurysm neck is larger in patients with stent graft migration. Eur J Vasc Endovasc Surg. 2010;40:326-31.

3. Thomas B, Sanchez L. Proximal migration and endoleak: Impact of endograft design and deployment techniques. Semin Vasc Surg. 2009;22:201-6.

4. Ilyas S, Shaida N, Thakor AS, Winterbottom A, Cousins C. Endovascular aneurysm repair (EVAR) follow-up imaging: the assessment and treatment of common postoperative complications. Clin Radiol. 2015;70:183-96.

5. Verhoeven ELGG, Mani K. New Technology Failures: Who to Blame or Time to be Cautious? Eur J Vasc Endovasc Surg. 2018;56:318-9.

6. Buck DB, van Herwaarden JA, Schermerhorn ML, Moll FL. Endovascular treatment of abdominal aortic aneurysms. Nat Rev Cardiol. 2014;11:112-23.

7. Hobo R, Buth J. Secondary interventions following endovascular abdominal aortic aneurysm repair using current endografts. A EUROSTAR report. J Vasc Surg. 2006;43:896-902.

8. Troisi N, Donas KP, Austermann M, Tessarek J, Umscheid T, Torsello G. Secondary procedures after aortic aneurysm repair with fenestrated and branched endografts. J Endovasc Ther. 2011;18:146-53.

9. De Bruin JL, Baas AF, Buth J, Prinssen M, Verhoeven ELG, Cuypers PWM, et al. Long-term outcome of open or endovascular repair of abdominal aortic aneurysm. N Engl J Med. 2010;362:1881-9.

10. Verhoeven ELG, Vourliotakis G, Bos WTGJ, Tielliu IFJ, Zeebregts CJ, Prins TR, et al. Fenestrated Stent Grafting for Short-necked and Juxtarenal Abdominal Aortic Aneurysm: An 8-Year Single-centre Experience. Eur J Vasc Endovasc Surg. 2010;39:529-36.

11. Roos JE, Hellinger JC, Hallet R, Fleischmann D, Zarins CK, Rubin GD. Detection of endograft fractures with multidetector row computed tomography. J Vasc Surg. 2005;42:1002-6.

12. Kansal V, Nagpal S, Jetty P. Editor's Choice - Late Open Surgical Conversion after Endovascular Abdominal Aortic Aneurysm Repair. Eur J Vasc Endovasc Surg. 2018;55:163-9.

13. Falkensammer J, Taher F, Uhlmann M, Hirsch K, Strassegger J, Assadian A. Rescue of failed endovascular aortic aneurysm repair using the fenestrated Anaconda device. J Vasc Surg. 2017;66:1334-9.

14. Langs G, Paragios N, Desgranges P, Rahmouni A, Kobeiter H. Learning deformation and structure simultaneously: In situ endograft deformation analysis. Med Image Anal. 2011;15:12-21.

15. Klein A, Renema W, Vliet JA, Oostveen LJ, Hoogeveen Y, Schultze Kool LJ, et al. Motion Calculations on Stent Grafts in AAA. In: Grundmann RT, editor. Diagnosis, Screening and Treatment of Abdominal, Thoracoabdominal and Thoracic Aortic Aneurysms. [Rijeka]: InTechOpen; 2011. p. $125-44$.

16. van Keulen JW, van Prehn J, Prokop M, Moll FL, van Herwaarden JA. Dynamics of the Aorta Before and After Endovascular Aneurysm Repair: A Systematic Review. Eur J Vasc Endovasc Surg. 2009;38:586-96.

17. Teutelink A, Muhs BE, Vincken KL, Bartels LW, Cornelissen SA, van Herwaarden JA, et al. Use of dynamic computed tomography to evaluate pre- and postoperative aortic changes in AAA 
patients undergoing endovascular aneurysm repair. J Endovasc Ther. 2007;14:44-9.

18. Iezzi R, Di Stasi C, Dattesi R, Pirro F, Nestola M, Cina A, et al. Proximal aneurysmal neck: dynamic ECG-gated CT angiography-conformational pulsatile changes with possible consequences for endograft sizing. Radiology. 2011;260:591-8.

19. van Keulen JW, Vincken KL, van Prehn J, Tolenaar JL, Bartels LW, Viergever MA, et al. The Influence of Different Types of Stent Grafts on Aneurysm Neck Dynamics after Endovascular Aneurysm Repair. Eur J Vasc Endovasc Surg. 2010;39:193-9.

20. Pol JA, Truijers M, van der Vliet JA, Fillinger MF, Marra SP, Renema WKJ, et al. Impact of dynamic computed tomographic angiography on endograft sizing for endovascular aneurysm repair. J Endovasc Ther. 2009;16:546-51.

21. Muhs BE, Vincken KL, van Prehn J, Stone MKC, Bartels LW, Prokop M, et al. Dynamic Cine-CT Angiography for the Evaluation of the Thoracic Aorta; Insight in Dynamic Changes with Implications for Thoracic Endograft Treatment. Eur J Vasc Endovasc Surg. 2006;32:532-6.

22. Ganten MK, Krautter U, Von Tengg-Kobligk H, Böckler D, Schumacher H, Stiller W, et al. Quantification of aortic distensibility in abdominal aortic aneurysm using ECG-gated multi-detector computed tomography. Eur Radiol. 2008;18:966-73.

23. de Beaufort HWL, Nauta FJH, Conti M, Cellitti E, Trentin C, Faggiano E, et al. Extensibility and Distensibility of the Thoracic Aorta in Patients with Aneurysm. Eur J Vasc Endovasc Surg. 2017;53:199-205.

24. Ganten M, Boese JM, Leitermann D, Semmler W. Quantification of aortic elasticity: Development and experimental validation of a method using computed tomography. Eur Radiol. $2005 ; 15: 2506-12$.

25. Klein A, Oostveen LJ, Greuter MJWW, Hoogeveen Y, Schultze Kool LJ, Slump CH, et al. Detectability of Motions in AAA With ECG-gated CTA: a Quantitative Study. Med Phys. 2009;36:4616-24.

26. QRM Quality assurance in Radiology and Medicine GmbH. Motion Simulator Sim2D. Available from: http://www.qrm.de/content/products/dynamic/sim2d.htm. Accessed 20 May 2015.

27. Flora HS, Woodhouse N, Robson S, Adiseshiah M. Micromovements at the aortic aneurysm neck measured during open surgery with close-range photogrammetry: Implications for aortic endografts. J Endovasc Ther. 2001;8:511-20.

28. Nichols WW. Clinical measurement of arterial stiffness obtained from noninvasive pressure waveforms. Am J Hypertens. 2005;18:3-10.

29. Klein A, van der Vliet JA, Oostveen LJ, Hoogeveen Y, Schultze Kool LJ, Renema WKJ, et al. Automatic segmentation of the wire frame of stent grafts from CT data. Med Image Anal. 2012;16:127-39.

30. Klein A, Kroon D-J, Hoogeveen Y, Schultze Kool LJ, Renema WKJ, Slump CH. Multimodal image registration by edge attraction and regularization using a B-spline grid. Proc SPIE Med Imaging. 2011;7962:796220-8.

31. Thompson M, Youssef M, Jacob R, Zerwes S, Reijnen M, Szopinski P, et al. Early Experience With Endovascular Aneurysm Sealing in Combination With Parallel Grafts for the Treatment of Complex Abdominal Aneurysms: The ASCEND Registry. J Endovasc Ther. 2017;24:764-772.

32. Schriefl AJ, Zeindlinger G, Pierce DM, Regitnig P, Holzapfel GA. Determination of the layerspecific distributed collagen fibre orientations in human thoracic and abdominal aortas and common iliac arteries. J R Soc Interface. 2012;9:1275-86.

33. Nasr B, Le Ven F, Savean J, Ben Salem D, Nonent M, Gouny P, et al. Characterization of the Physiological Displacement of the Aortic Arch Using Non-Rigid Registration and MR Imaging. Eur J Vasc Endovasc Surg. 2017;53:282-9. 


\section{Appendix A}

The following are the Supplementary data to this chapter:

Supplemental Resource 1: Aortic motion patterns and motion device validation. Supplemental Resource 2: Motion modeling by registration and segmentation. Supplemental Video S1, S2, S3.

Supplemental videos can be accessed online:

https://doi.org/10.1016/j.ejvs.2019.03.009

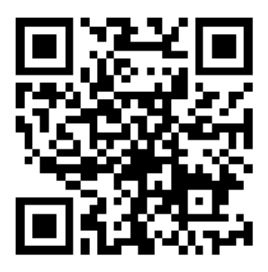




\section{Supplemental Resource 1: Aortic motion patterns and motion device validation}

Detailed information on the generated motion patterns and the accuracy and precision of the motion device is provided in this supplementary document. Video S1 and S2 are complementary to this document.

Abdominal aortic motion patterns A motion pattern composed of Gaussian functions was designed to mimic aortic motion and to control the pattern's frequency and amplitude:

$$
\begin{gathered}
P(t)=A \times\left(G_{\sigma 1}\left(t-t_{\text {top }}\right) \times H\left(t_{\text {top }}-t\right)+G_{\sigma 2}\left(t-t_{\text {top }}\right) \times H\left(t-t_{\text {top }}\right)+P_{\text {extra }}(t)\right)(1) \\
G_{\sigma}(t)=e^{-t^{2} / 2 \sigma^{2}} \\
H(t)=\left\{\begin{array}{c}
1, t>0 \\
0, t \leq 0
\end{array}\right. \\
P_{\text {extra }}(t)=A_{\text {extra }} \times G_{\sigma_{\text {extra }}}\left(t-t_{\text {top extra }}\right)
\end{gathered}
$$

where $P(t)$ is the position over time $(0 \leq \mathrm{t} \leq \mathrm{T}$ [period]), $A$ is the amplitude of the pattern, $G_{\sigma}(t)$ (Equation 2) and $H(t)$ (Equation 3) the Gaussian and Heaviside functions respectively, $\sigma 1$ and $\sigma 2$ the sigma's of the respective Gaussian function, and $t_{\text {top }}$ the temporal location of the peak. Both sigma and peak location are expressed as a fraction of the period $(\mathrm{T})$. A second peak can be created in the pattern by setting $A_{\text {extra }}, t_{\text {top extra }}$ and $\sigma_{\text {extra }}$ to nonzero (Equation 4 ).

Two series of motion patterns (Table S1.1) were generated using Equation1, with $t_{\text {top }}$ at $35 \%$ of the period, which represents the peak systole in the abdominal aorta[1], and with $\sigma_{1}$ of $0.4 \times t_{t o p}$ and $\sigma_{2}$ of $0.4 \times\left(1-t_{t o p}\right)$. In the $\mathrm{A}$ series the heart rate was changed while the amplitude remained constant and in the $\mathrm{B}$ series vice versa. For the A series the output amplitude of the motion device was tuned to be constant. The pattern B0 contained no motion and was included as a control. Two of the generated patterns are presented in Figure S1.1. Note that with amplitude we refer to the maximum absolute value of displacement, that is, the maximum distance between two positions during a full cycle. Two motion patterns were given a second peak at $80 \%$ of the period to simulate irregular aortic motion, resembling wave reflection patterns[2] and non-uniform patterns such as reported by Flora et al.[3]. The amplitude of motion in the abdominal aorta has been reported to be in the order of 1-2 $\mathrm{mm}[3,4]$. Amplitudes for the motion patterns generated were chosen relatively small since the proximal sealing rings of the stent graft are expected to locally decrease the motion of the abdominal aorta.

Accuracy and precision of the motion device The motion device (PC controlled phantom device, QRM Quality Assurance in Radiology and Medicine GmbH, Möhrendorf, Germany[5]) provides 1D motions according to preset patterns with a maximum amplitude of $50 \mathrm{~mm}$. To be able to evaluate the accuracy of the proposed algorithm for measuring stent graft motion in ECG-gated CT data it is of paramount 
Table S1.1. Patterns generated with the motion device. Input (Equation 1) and output parameters of the executed patterns with mean and standard deviation are given. The actual executed patterns were used as a reference for algorithm validation. Note that A3 and B3 are the same pattern.

\begin{tabular}{lccccc}
\hline Motion Pattern & \multicolumn{3}{c}{ Input } & \multicolumn{2}{c}{ Output } \\
& $F(\mathrm{bpm})$ & $A(\mathrm{~mm})$ & $A_{\text {extra }}, \sigma_{\text {extra }}$ & $F(\mathrm{bpm})$ & $A(\mathrm{~mm})$ \\
\hline A1 & 50 & 1.0 & - & $49 \pm 1$ & $0.72 \pm 0.01$ \\
A2 & 100 & 1.3 & - & $96 \pm 2$ & $0.75 \pm 0.01$ \\
A3 & 75 & 1.2 & - & $73 \pm 1$ & $0.70 \pm 0.01$ \\
B0 & 75 & 0 & - & 75 & 0 \\
B1 & 75 & 0.6 & - & $73 \pm 1$ & $0.23 \pm 0.02$ \\
B2 & 75 & 0.8 & - & $73 \pm 1$ & $0.37 \pm 0.02$ \\
B3 & 75 & 1.2 & - & $73 \pm 1$ & $0.70 \pm 0.01$ \\
B4 & 75 & 2.0 & - & $73 \pm 1$ & $1.38 \pm 0.05$ \\
B5 & 75 & 2.0 & $0.7,0.05$ & $73 \pm 1$ & $1.26 \pm 0.04$ \\
B6 & 75 & 2.0 & $0.7,0.09$ & $73 \pm 1$ & $1.24 \pm 0.02$ \\
\hline A & 75 &
\end{tabular}

Abbreviations: $F$, frequency in beats per minute (bpm); $A$, amplitude in millimeters.

A

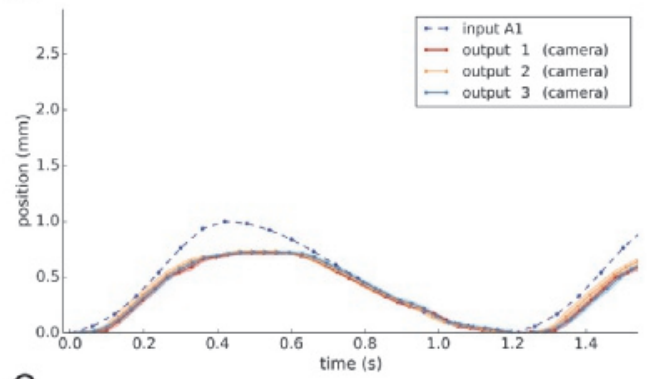

C

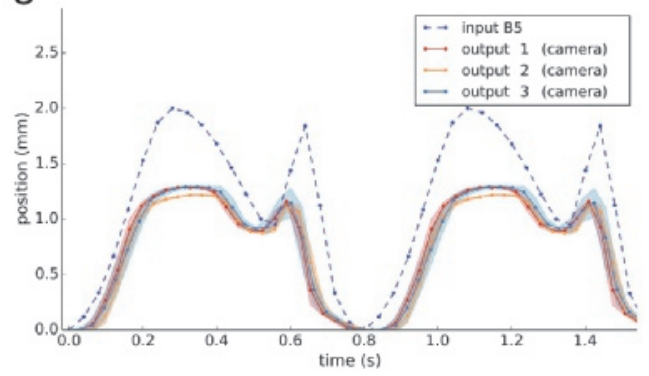

B

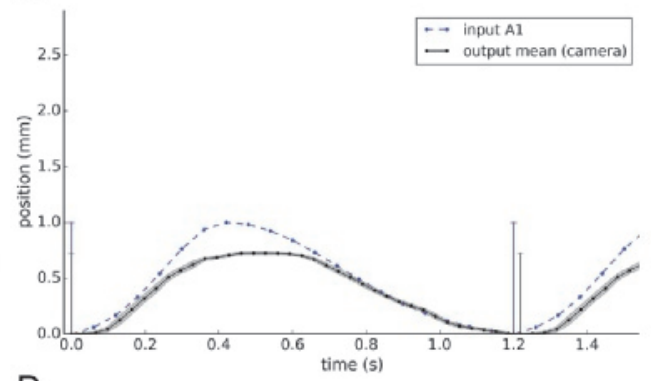

$\mathrm{D}$

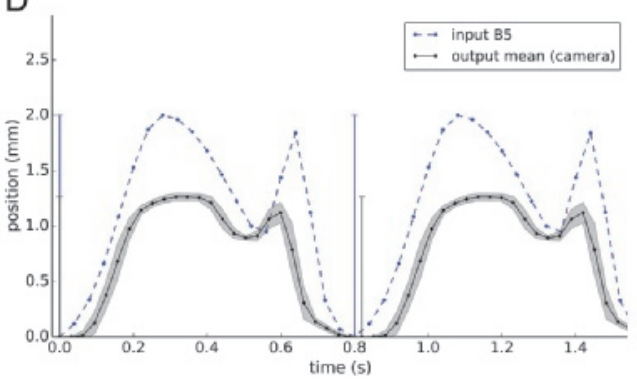

Figure S1.1. Example of motion pattern A1 and B5 (Table S1.1). 'Input' refers to the patterns that were programmed in the motion device. 'Output' refers to the actual executed patterns as obtained by analysis of optical camera recordings. The mean \pm standard deviation (colored bounds) of the recorded periods is shown periodically. The three camera recordings ('output' in $\mathrm{A}, \mathrm{C}$ ) were averaged to obtain a reference ('output mean' in B,D) for algorithm validation. The height of the vertical lines denotes the amplitude of the respective pattern. These lines also indicate the start and end of the period. 
A

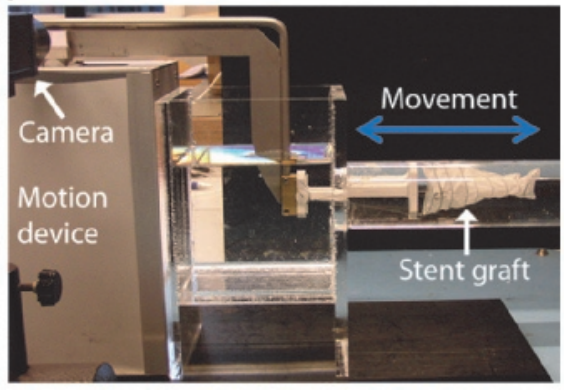

B

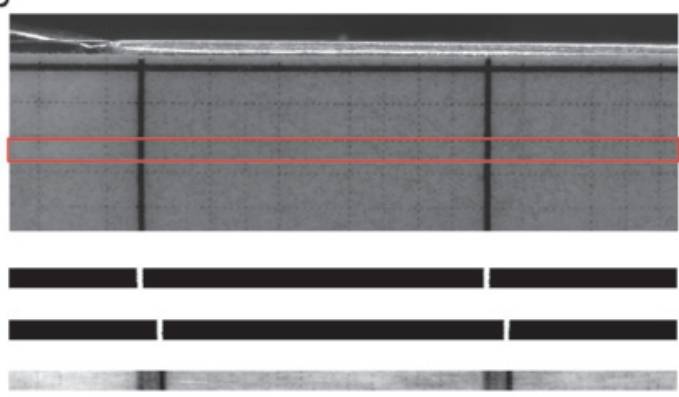

Figure S1.2. The experimental setup with a camera recording a mm-grid on the lever of the motion device moving in $z$-direction (A; Video S1). Displacement of the grid lines was analyzed by applying a binary threshold operation on a section of the recorded grid (red rectangle) in Grayscale image frames (B; Video S2). The thresholded section (black and white) is shown for a frame at the start and end of a period. The average of all recorded image frames is also shown to visualize displacement of the grid lines (bottom). The obtained displacement patterns (Fig. S1.1) were used as a reference for algorithm validation.

importance that the motion executed by the device is known and precise. Technical specifications of the device include an accuracy $<6.3 \%$ for a sinusoidal motion at 140 beats per minute $(\mathrm{bpm})$ and an accuracy $<0.2 \mathrm{~mm}$ in static mode (constant displacement) better than $0.2 \mathrm{~mm}$. However, these specifications were not guaranteed for the relatively small motion patterns of interest. To obtain a sub-mm accurate ground truth for algorithm validation, we have analyzed the executed motion by optical camera recordings of the experimental setup (Figure S1.2). A Sony XCD-X710 high resolution monochrome camera (30 frames/s; XGA: $1024 \times 768$ pixels) recorded the displacement of an attached mm-grid. Each pattern was recorded three times for approximately 20 seconds, which is similar to the duration of an ECG-gated CT scan. These recordings were alternated with the experimental CT scans of the setup because simultaneous measurements during CT acquisition were not feasible. The displacement of mm-grid lines in the images was analyzed in Matlab 2014b (The Mathworks Inc., Natick, MA, USA) by converting the images to grayscale and applying a binary threshold operation (Figure S1.2). To obtain a reference for each pattern, the average over all recorded periods was calculated. Individual periods, i.e., waveform segments, were obtained from the signals by detecting the local minima. Subsequently, these segments were superimposed by time shifting while minimizing the mean squared error, and averaged. Average waveform amplitudes and the variation of amplitudes are presented in Table S1.1 (Output) together with the programmed motion (Input). The variation in amplitude was small for all patterns, with standard deviations between $0.01 \mathrm{~mm}$ and $0.05 \mathrm{~mm}$. There may have been several reasons for the small variations between waveform segments, including the motion device itself, water resistance, and the processing of camera images. In Figure S1.1 the programmed patterns (Input) and the actual executed patterns (Output) are shown for two of the generated motion patterns.

In sum, analysis of the motion device showed that the executed motion devi- 
ated from the programmed motion but the motion was precise and reproducible in $z$-direction (superior-inferior) and was therefore considered a reliable reference for validation of the algorithm. For each motion pattern the mean of the recorded periods, i.e., one full cycle, was used as the reference. In $x$-direction (right-left), with the lever rotated by 90 degrees, the motion was not consistent for the small motion patterns of interest. Experiments were therefore performed in $z$-direction only.

\section{References}

1. Hazer D, Finol EA, Kostrzewa M, Kopaigorenko M, Richter G-M, Dillmann R. Computational biomechanics and experimental validation of vessel deformation based on 4D-CT imaging of the porcine aorta. Hu XP, Clough A V., editors. Proc SPIE Med Imaging. 2009;7262:72621F-9.

2. Nichols WW. Clinical measurement of arterial stiffness obtained from noninvasive pressure waveforms. Am J Hypertens. 2005;18:3-10.

3. Flora HS, Woodhouse N, Robson S, Adiseshiah M. Micromovements at the aortic aneurysm neck measured during open surgery with close-range photogrammetry: Implications for aortic endografts. J Endovasc Ther. 2001;8:511-20.

4. van Keulen JW, van Prehn J, Prokop M, Moll FL, van Herwaarden JA. Dynamics of the Aorta Before and After Endovascular Aneurysm Repair: A Systematic Review. Eur J Vasc Endovasc Surg. 2009;38:586-96.

5. QRM Quality assurance in Radiology and Medicine GmbH. Motion Simulator Sim2D. Available from: http://www.qrm.de/content/products/dynamic/sim2d.htm. Accessed 20 May 2015. 


\section{Supplemental Resource 2: Motion modeling by registration and segmentation}

Motion of the stent-graft was quantitatively assessed by applying a combined image registration and segmentation algorithm in which deformation fields are applied to a stent-graft model to estimate its position at different time points in the cardiac cycle[1-3]. The process of registration and segmentation is explained in detail in the following section, including applied parameters and modifications that were implemented in the present work. An overview of the applied methodology is shown in Figure 2 in chapter 6.

Registration algorithm Deformable (i.e., elastic) registration of the time resolved $3 \mathrm{D}$ volumes was applied in a groupwise fasion, whereby the volumes are simultaneously (i.e., groupwise) registered to each-other towards their 'mean shape' (in order not to be biased towards a single volume). This results in a deformation (i.e., vector) field for each volume, and (by averaging the registered volumes) a 3D reference volume representing this mean shape, hereafter referred to as the time-averaged volume. A deformation field therefore describes the displacement of each voxel in the volume with respect to the time-averaged volume.

The registration algorithm combines a B-spline grid for regularization with an image 'force' that drives the registration from individually weighted voxels. It was first proposed by Klein et al.[4] and is published within the Python Image Registration Toolkit (PIRT) in a Bitbucket repository. The algorithm uses a so called 'Gravity force' to drive the registration, which is based on the idea of masses, i.e., pixels/voxels with high intensities, in the images/volumes that attract one another. There are two parameters that are specific to this registration algorithm; the SpeedFactor determines the relative strength of the deformation at each iteration and the MassDerivativeOrder defines how the images are transformed at each iteration to obtain mass images in order to detect changes of mass between the registered images. The MassDerivativeOrder refers to the order of differentiation where 1 and 2 refer to the gradient magnitude and the Laplacian respectively. Here we have used a Laplacian operator for the calculation of mass images, which gives a high response to second order derivatives (e.g., lines in 3D), such that the resulting mass (and attraction thereof) is dominated by the wires of the stent-graft. The registration is an iterative process in which the scale is smoothly decreased until the FinalScale is reached. The ScaleSampling sets the number of iterations per scale reduction. The regularization of the deformations is controlled by the parameter FinalGridSampling, which specifies the spacing between the knots of the final B-spline grid. Small values allow for more variation in the deformation but decrease the level of regularization. Parameter settings for the present work are given in Table S2.1 and are based on qualitative evaluation of the deformed volumes and previous experimental work[2].

Segmentation algorithm Geometric models of the stent-rings were obtained from the time-averaged volumes by applying the segmentation algorithm described by Klein et al.[3]. In short, this method can be divided into three steps in which several parameters are used. Parameter values employed for the present work were determined 
experimentally for the time-averaged volumes of one motion pattern and were reused for the other volumes (Table S2.1). First, seed points on the wire frame of the stentgraft are detected by finding local maxima with an intensity above the SeedThreshold and a direct neighbor with an intensity that is also above this threshold (step 1). Second, a Minimum Cost Path (MCP) method is used to trace the wire and connect the seed points by growing fronts from all the seed points (step 2). The parameter MCPspeedfactor determines the cost function, i.e., the algorithms' affinity for high intensities. This step results in a graph that represents the wire frame and consists of nodes (seed points) and edges with an associated 'cumulative cost' and intensity (in Hounsfield units [HU's]). Third, a graph processing operation is performed to iteratively prune (i.e., remove) unwanted nodes and edges and to reposition the nodes at edge crossings (step 3). In this process the algorithm removes all edges with a HU below the WeakThreshold, preserves edges with a HU above the StrongThreshold, and removes redundant edges with $\mathrm{HU}$ in between these threshold values.

The pruning operation was modified to retain the hook struts between the two upper ring stents of the Anaconda stent-graft. Furthermore, we have implemented a subvoxel estimation for the locations of nodes and edges in the geometric model using 1D quadratic polynomials to fit the $\mathrm{x}, \mathrm{y}$ and $\mathrm{z}$ subvoxel location, to position the points in the center of the wire bundle while obtaining smoother models.

Table S2.1. Parameter settings used for image registration and segmentation.

\begin{tabular}{llll}
\hline \multicolumn{1}{c}{ Registration } & Segmentation & \\
Parameter & Value & Parameter & Value \\
\hline MassDerivativeOrder & 2 & SeedThreshold (step 1) & 2000 [HU] \\
SpeedFactor & 1.0 & MCPspeedfactor (step 2) & 500 \\
ScaleSampling & 16 & WeakThreshold (step 3) & $500[\mathrm{HU}]$ \\
FinalGridSampling & 20 & StrongThreshold (step 3) & 1800 [HU] \\
FinalScale & 1.0 & & \\
\hline Abbreviation & & \\
\hline
\end{tabular}

Abbreviations: HU, Hounsfield units

\section{References}

1. Klein A, Renema W, Vliet JA, Oostveen LJ, Hoogeveen Y, Schultze Kool LJ, et al. Motion Calculations on Stent Grafts in AAA. In: Grundmann RT, editor. Diagnosis, Screening and Treatment of Abdominal, Thoracoabdominal and Thoracic Aortic Aneurysms. [Rijeka]: InTechOpen; 2011. p. 125-44.

2. Klein A. A tool for studying the motion of stent grafts in AAA. Segmentation and motion estimation of stent grafts in abdominal aortic aneurysms. [Enschede]: PhD Dissertation, University of Twente; 2011. p. 121-37.

3. Klein A, van der Vliet JA, Oostveen LJ, Hoogeveen Y, Schultze Kool LJ, Renema WKJ, et al. Automatic segmentation of the wire frame of stent grafts from CT data. Med Image Anal. 2012;16:127-39.

4. Klein A, Kroon D-J, Hoogeveen Y, Schultze Kool LJ, Renema WKJ, Slump CH. Multimodal image registration by edge attraction and regularization using a B-spline grid. Proc SPIE Med Imaging. 2011;7962:796220-8. 


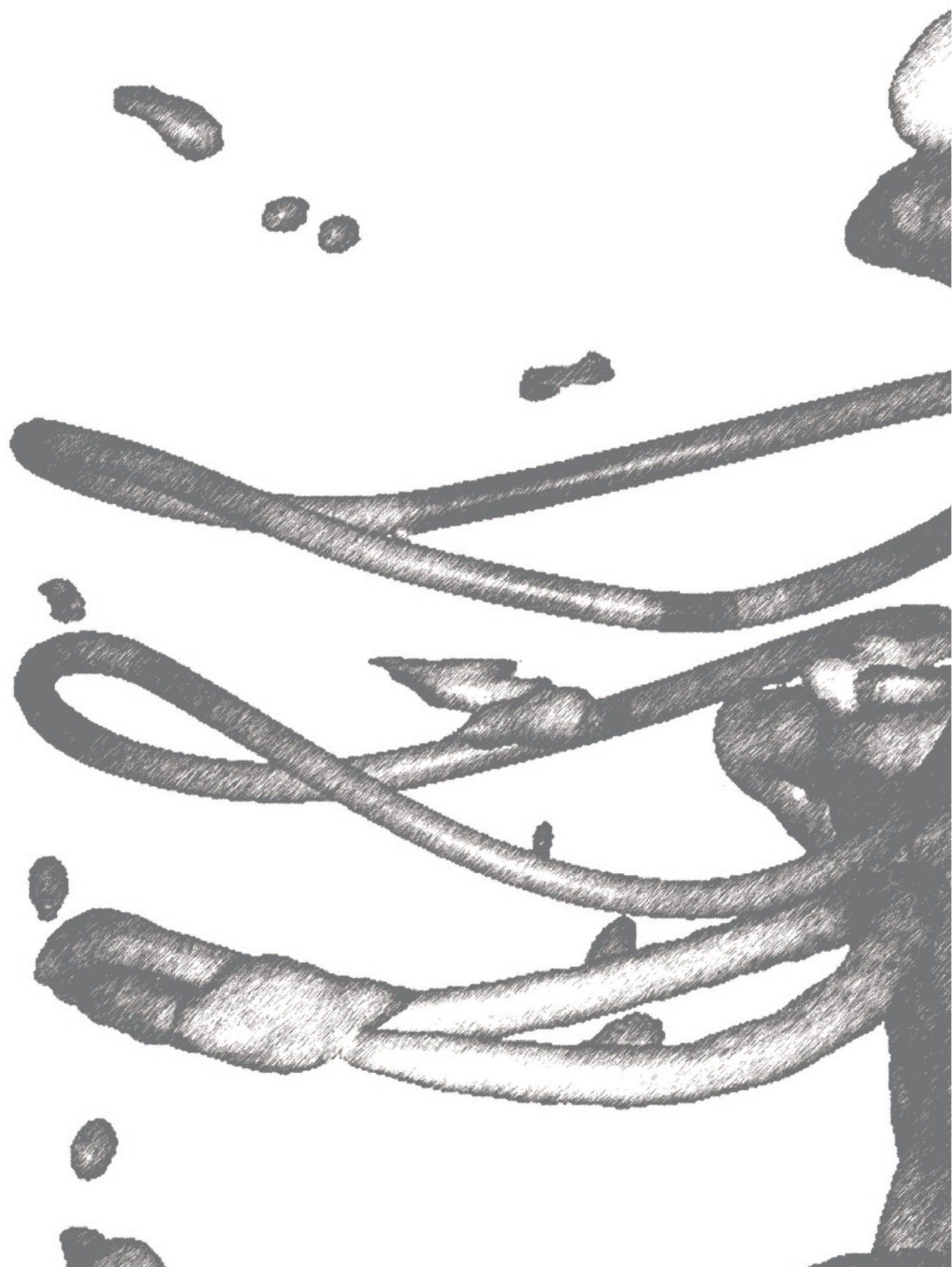



\section{Chapter 7}

\section{Electrocardiogram gated computed tomography quantification of sealing ring dynamics after endovascular aneurysm repair in patients treated with an Anaconda stent-graft}

Maaike A. Koenrades ${ }^{a, b, c}$, Almar Klein ${ }^{d}$, Cornelis H. Slump ${ }^{c}$, and Robert H. Geelkerken ${ }^{a, b}$

${ }^{a}$ Multi-modality Medical Imaging (M3I) group, Faculty of Science and Technology, Technical Medical Centre, University of Twente, Enschede, the Netherlands

${ }^{b}$ Department of Vascular Surgery, Medisch Spectrum Twente, Enschede, the Netherlands

${ }^{c}$ Robotics and Mechatronics (RaM) group, Faculty of Electrical Engineering, Mathematics and Computer Science, Technical Medical Centre, University of Twente, Enschede, the Netherlands

${ }^{d}$ Independent Scholar, Enschede, the Netherlands 


\section{Abstract}

Purpose: To evaluate the cardiac pulsatility-induced motion and deformation of the Anaconda stent-graft double sealing ring.

Methods: Postoperative electrocardiogram gated computed tomography scans of 15 patients were evaluated using dedicated image-processing algorithms. The scans were prospectively gathered by study protocol before discharge and 1, 6, 12, and 24 months after endovascular aneurysm repair. After deformable registration of the reconstructed phases, the proximal sealing rings were segmented to study the in situ stent-ring displacement, pulsatile distension, and curvature change during the cardiac cycle at each time point.

Results: There were no significant cardiac pulsatility-induced changes between time points during follow-up. Displacements up to $0.9 \mathrm{~mm}$ in $\mathrm{x}$ - and $\mathrm{y}$-direction and up to $1.6 \mathrm{~mm}$ in z-direction were measured during the cardiac cycle. Pulsatile distension was below $1 \mathrm{~mm}(<3.5 \%)$, with asymmetry ratios ranging from 1.0 to 4.7 . The distance between the first and second stent-ring varied by at most $0.4 \mathrm{~mm}$ during the cardiac cycle. Maximum change in curvature was at most $0.148 \mathrm{~cm}^{-1}(6 \%)$ for the first stent-ring and $0.218 \mathrm{~cm}^{-1}(8 \%)$ for the second stent-ring; mostly located near the peaks and valleys of the saddle-shaped stent-rings. Changes in curvature were most pronounced at the anterior quadrant of the stent-rings and smallest at the posterior quadrant.

Conclusion: The proximal stent-rings adequately counteracted aortic pulsatility to reduce the risk of stent fatigue. The presented methodology enables to identify in-vivo stent locations that may have a lower fatigue safety factor under in-vivo loading conditions, and are therefore of particular importance when considering device durability.

Keywords: Endovascular aortic aneurysm repair, electrocardiogram gated computed tomography, nitinol ring, fatigue, pulsatile distension, dynamics, Anaconda stentgraft, proximal fixation and sealing 


\section{Introduction}

Advancements in preclinical testing and design verification of stent-grafts have substantially reduced the failure incidence of endovascular aortic aneurysm repair (EVAR)[1]. Still, device fatigue remains a major concern as it can lead to late rupture of abdominal aortic aneurysm (AAA)[2]. While fractures of the wire frame may often remain unnoticed as they are typically asymptomatic, instances of stent fracture have certainly been observed[2,3]. The long-term durability of stent-grafts is difficult to assess as it depends on a multitude of factors related to the device design and the patient-specific endovascular environment[1]. Computational models and accelerated fatigue tests are helpful to understand the distribution of stress and strain to estimate stent life but depend on the applied boundary conditions[1,4-6]. Knowledge of the dynamics of implanted stent-grafts is therefore crucial in the design and development of stent-grafts for input into stress-strain analysis, fatigue life evaluation and design verification. In addition, determining the extent of stent-graft radial distension and displacement due to pulsatile blood flow may further aid our understanding of factors contributing to stent-graft dislocation and type Ia endoleak $[7,8]$.

The Anaconda stent-graft is a trimodular device with two proximal stent-rings for sealing interconnected by four pairs of hooks between the stent-rings to provide active fixation. The stent-rings consist of multiple turns of Nitinol wire that form a wire bundle. The design philosophy behind this device is that the proximal stent-rings should be sufficiently stiff to counteract the pulsatility of the aortic vessel during the cardiac cycle and thus minimize the risk of metal fatigue by reducing the cardiacinduced cyclic deformation of the Nitinol stent material to a level below its fatigue safety threshold. Even though the Anaconda device has been shown to perform well in clinical use[9-17], empirical evidence of in situ stent-ring dynamics during the cardiac cycle is lacking. For this reason, the present study evaluates motion and deformation of the proximal stent-rings using electrocardiogram (ECG)-gated computed tomography and dedicated image-processing algorithms.

\section{Methods}

Patient sample The patient data from the Longitudinal Study of Pulsatility and Expansion in Aortic Stent-grafts (Trialregister.nl identifier NTR4276) were used to further quantify sealing ring dynamics during the cardiac cycle in 15 patients (mean age $72.8 \pm 3.7 ; 14$ men) treated with an Anaconda stent-graft (mean oversizing $31 \pm 9 \%$ ) for an infrarenal AAA between April 2014 and May 2015. According to protocol, patients were prospectively followed for 2 years after EVAR by noncontrast ECGgated computed tomography (CT) scans before discharge (within 3 days) and after $1,6,12$, and 24 months of follow-up. Two of the 15 patients were followed up to 12 months due to one voluntary withdrawal and one non-AAA related death. The trial design and patient sample were reported in a previous study about longitudinal expansion of the sealing rings[18]. The study design and protocol were approved by the institutional review board. Informed consent was obtained from all participants. 

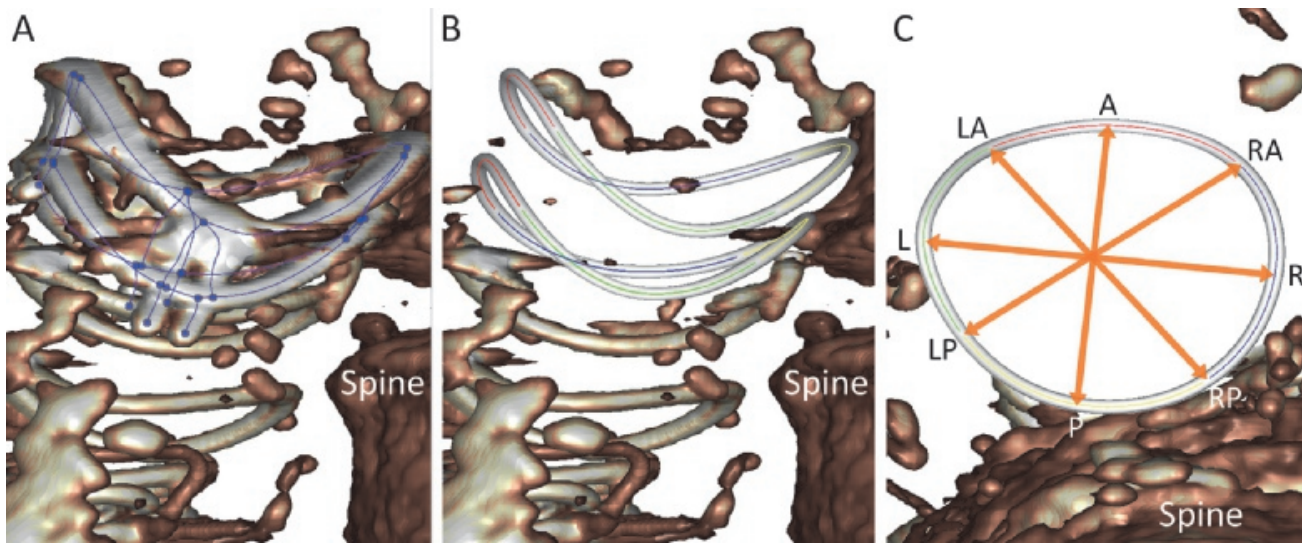

Figure 1. Illustration of quantitative measurements of the stent-ring models. First, the hooks were removed from the model to obtain the stent-rings individually (A, B). The stentrings were subdivided into four quadrants (colors in B, C: yellow, posterior; green, left; red, anterior; blue, right) to quantify displacement, distance change between the first and second stent-ring, and curvature change. Pulsatility (distance max $_{\text {- }}$ distance $_{\min }$ ) was measured over four directions of distension, from peak-to-peak, valley-to-valley, and between the points halfway in between the peaks and valleys, i.e., mid-to-mid (C). The top view visualizes the first stent-ring only (C). P, posterior; A, anterior; L, left; R, right; LP, left posterior; RP, right posterior; LA, left anterior; RA, right anterior.

Imaging All scans were performed on an Aquilion 64 CT scanner (Toshiba Medical Systems Corporation, Tokyo, Japan) or on a Somatom Definition Flash CT scanner (Siemens Healthineers, Forchheim, Germany) with a standardized low-dose scan protocol based on the routine static protocol for the abdomen. The 24-month scans were exclusively acquired on the Somatom Flash scanner. Scans parameters were rotation time, 0.4 seconds (Aquilion) and 0.3 seconds (Flash); collimation, $64 \times 0.5 \mathrm{~mm}$ (Aquilion) and $2 \times 128 \times 0.6 \mathrm{~mm}$ (Flash); slice thickness, $1 \mathrm{~mm}$; slice increment, $0.5 \mathrm{~mm}$; reconstructed matrix size, $512 \times 512$ pixels. The pitch factor was set automatically based on the heart rate. Radiation exposure parameters were $120 \mathrm{kV}$ and 40, 60, or $80 \mathrm{~mA} \cdot \mathrm{s}$ based on the patient's body mass index $\left(<20,20-25,>25 \mathrm{~kg} / \mathrm{m}^{2}\right.$, respectively), resulting in a dose length product of $962.1 \pm 220.1 \mathrm{mGy} \cdot \mathrm{cm}$ for a scan length of $\sim 30 \mathrm{~cm}$. Blood pressure was measured immediately after scanning with a digital brachial sphygmomanometer. Retrospective gating was performed at $10 \%$ intervals from $0 \%$ to $90 \%$ of the RR-interval covering the cardiac cycle.

Image analysis A previously developed algorithm dedicated to motion estimation of stent-grafts in multiphasic ECG-gated CT data[19-21] was used to quantify stentring dynamics during the cardiac cycle. First, the reconstructed volumes of each CT scan were simultaneously registered using a deformable (i.e., non-rigid) B-spline registration algorithm to obtain discrete deformation fields for each volume. The deformation fields describe the displacement of all voxels in the volume with respect to the average of all phases, hereafter referred to as time-averaged volume. The time- 
averaged volume represents mid cardiac cycle. Subsequently, the proximal sealing rings were segmented as reported previously[18]. To analyze stent-ring motion during the cardiac cycle, the deformation fields were applied to the stent-ring models by backward mapping to estimate the positions at each phase in the cardiac cycle with respect to the time-averaged volume. Applying this image registration technique avoids the need to repeat the segmentations in the different phases of the cardiac cycle, which avoids inconsistencies between the models in different phases and allows for automated comparison of phases.

The stent-ring models were then used to quantify displacement in $\mathrm{x}^{-}, \mathrm{y}-$ and z-direction, the change in distance between the first and second stent-ring, pulsatile distension (hereafter referred to as pulsatility), and the change in curvature during the cardiac cycle as illustrated in Figure 1. Individual models of the first and the second stent-ring were obtained by automatically detecting and removing the hooks in the model that interconnect the two stent-rings. Pulsatility was defined as the maximum (peak-systolic) minus the minimum (end-diastolic) diametric distance during the cardiac cycle. Mean and maximum pulsatility over four directions of distension were obtained as well as asymmetry ratios between perpendicular directions. To consider pulsatility under physiological pressure conditions, the change in diametric distance was calculated for a given change in pressure, i.e., compliance[22]. Compliance was computed as the percentage change in diametric distance per $100 \mathrm{mmHg}$ :

$$
\mathrm{C}=100 \times \frac{D_{\text {maximum }}-D_{\text {minimum }}}{D_{\text {minimum }}} \times \frac{100}{B P_{\text {systolic }}-B P_{\text {diastolic }}},
$$

where $\mathrm{C}$ is compliance, $\mathrm{D}$ the diametric distance and $\mathrm{BP}$ the blood pressure in $\mathrm{mmHg}$.

In order to characterize local deformation of the stent-rings, which can occur due to bending of the wire during radial vessel expansion and contraction, the change in curvature during the cardiac cycle was assessed for each point of the stent-ring models to obtain the maximum and mean change in curvature. Curvature was defined for a parametrically-defined 3-dimensional (3D) curve:

$$
\kappa=\frac{\sqrt{\left(z^{\prime \prime} y^{\prime}-y^{\prime \prime} z^{\prime}\right)^{2}+\left(x^{\prime \prime} z^{\prime}-z^{\prime \prime} x^{\prime}\right)^{2}+\left(y^{\prime \prime} x^{\prime}-x^{\prime \prime} y^{\prime}\right)^{2}}}{\left(x^{\prime 2}+y^{\prime 2}+z^{\prime 2}\right)^{3 / 2}}
$$

where $\mathrm{x}(n), \mathrm{y}(n)$, and $\mathrm{z}(n)$ are the Cartesian coordinates of a geometric stent-ring model in three dimensions for the number of points $n$ along the stent-ring model, ' is the first derivative and "the second derivative. The curvature implementation was validated using a helix-shaped phantom with a known curvature value[23].

Statistical analysis Data are presented as mean \pm standard deviation (range) for normally distributed data or as median [first quartile, third quartile] for nonparametric data. For comparison of values over time and between groups (i.e., directions of distension or stent-ring quadrants), a within-subjects one-way analysis of variance (ANOVA) was used for parametric data and the Friedman test with post hoc Wilcoxon signed-rank tests for non-parametric tests. Bonferroni adjustment for multiple comparisons was applied. P-values $<0.05$ were considered statistically sig- 
nificant. Statistical analysis was performed using SPSS Statistics (version 24.0; IBM Corporation, Armonk, NY, USA).

\section{Results}

The results are summarized in Table 1 and 2. Figure 2 summarizes the mean displacement in $\mathrm{x}-, \mathrm{y}-$, and $\mathrm{z}$-direction for the full stent-ring and per stent-ring quadrant. Figure 3 displays the maximum pulsatility for each patient during follow-up. Figure 4 summarizes pulsatility along the directions of distension as indicated in Figure 1. The change in curvature during the cardiac cycle is summarized for the full stent-rings and per stent-ring quadrant in Figure 5. Visualization of pulsatility and curvature change during the cardiac cycle of a case example is shown in Figure 6. A dynamic visualization of the original ECG-gated CT scan and color-coded representations of the stent-ring models are shown in Video S1, S2 and S3).

Displacement of the stent-rings During follow-up, displacement of the stentrings did not significantly change over time $(\mathrm{p}>0.069)$. Overall, displacements in $\mathrm{x}-, \mathrm{y}-$ and $\mathrm{z}$-direction and in $3 \mathrm{D}(\mathrm{p}>0.069)$ were on average $0.4 \pm 0.2 \mathrm{~mm}(0.1-0.9$ $\mathrm{mm}), 0.4 \pm 0.1 \mathrm{~mm}(0.1-0.8 \mathrm{~mm}), 0.7 \pm 0.3 \mathrm{~mm}(0.1-1.6 \mathrm{~mm})$, and $0.9 \pm 0.3 \mathrm{~mm}$ $(0.3-1.8 \mathrm{~mm})$ for the first stent-ring and $0.4 \pm 0.2 \mathrm{~mm}(0.1-0.9 \mathrm{~mm}), 0.4 \pm 0.1 \mathrm{~mm}$ $(0.1-0.7 \mathrm{~mm}), 0.7 \pm 0.3 \mathrm{~mm}(0.1-1.5 \mathrm{~mm})$, and $0.9 \pm 0.3 \mathrm{~mm}(0.3-1.7 \mathrm{~mm})$ for the second stent-ring, respectively. At most time points, displacements at the posterior quadrant of the stent-rings were significantly less compared to displacements at the other quadrants (Figure 2). Furthermore, displacement differed significantly between the left and right quadrant in $y$-direction at 24 months at both stent-rings $(\mathrm{p}<0.015)$ and between the anterior and right quadrant in $y$-direction at 24 months $(p=0.015)$ at the first stent-ring and at 1, 6, 12, and 24 months at the second stent-ring $(\mathrm{p}<0.009)$.

Over time, the mean change in distance between the first and second stent-ring varied by at most $0.4 \mathrm{~mm}$ during the cardiac cycle. There were no significant differences between time points $(\mathrm{p}>0.330)$ nor quadrants $(\mathrm{p}>0.160)$. Overall, the change in distance was on average $0.1 \pm 0.1 \mathrm{~mm}(0.0-0.4 \mathrm{~mm})$.

Pulsatility and curvature change of the stent-rings During follow-up CT examinations, there was no significant change in systolic blood pressure $(\mathrm{p}>0.106)$. A significant increase in diastolic pressure was noted between discharge and 12 months $(76.9 \pm 10.5 \mathrm{mmHg}$ vs. $87.7 \pm 12.0 \mathrm{mmHg}, \mathrm{p}=0.035)$. No significant differences in mean and maximum pulsatility and compliance were noted between time points during follow-up for both stent-rings $(\mathrm{p}>0.661)$. During follow-up, maximum pulsatility of the first and the second stent-ring were on average $0.4 \pm 0.2 \mathrm{~mm}(1.6 \pm 0.6 \%)$ and $0.4 \pm 0.2 \mathrm{~mm}(1.4 \pm 0.6 \%)$, respectively. There were no significant differences in pulsatility between the directions of distension $(\mathrm{p}>0.177)$, except for one significant difference between the anterior-posterior (AP) and the right anterior-left posterior direction (RALP) direction on the first stent-ring at 24 months of follow-up (0.34 \pm 0.11 vs. $0.21 \pm 0.11, \mathrm{p}<0.001)$. During follow-up, the pulsatility ratio ranged from 1.0 to 3.7 over the AP- vs. left-right (LR) direction and from 1.0 to 4.7 over the left 
R1
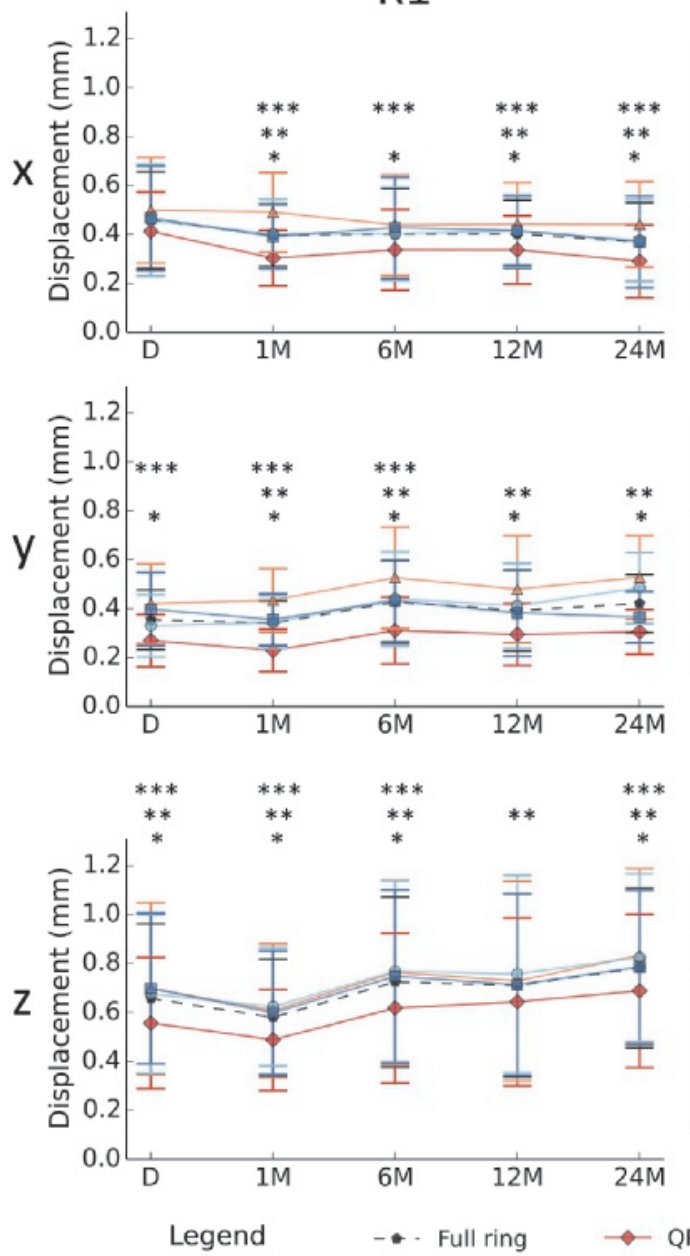

$\mathrm{R} 2$
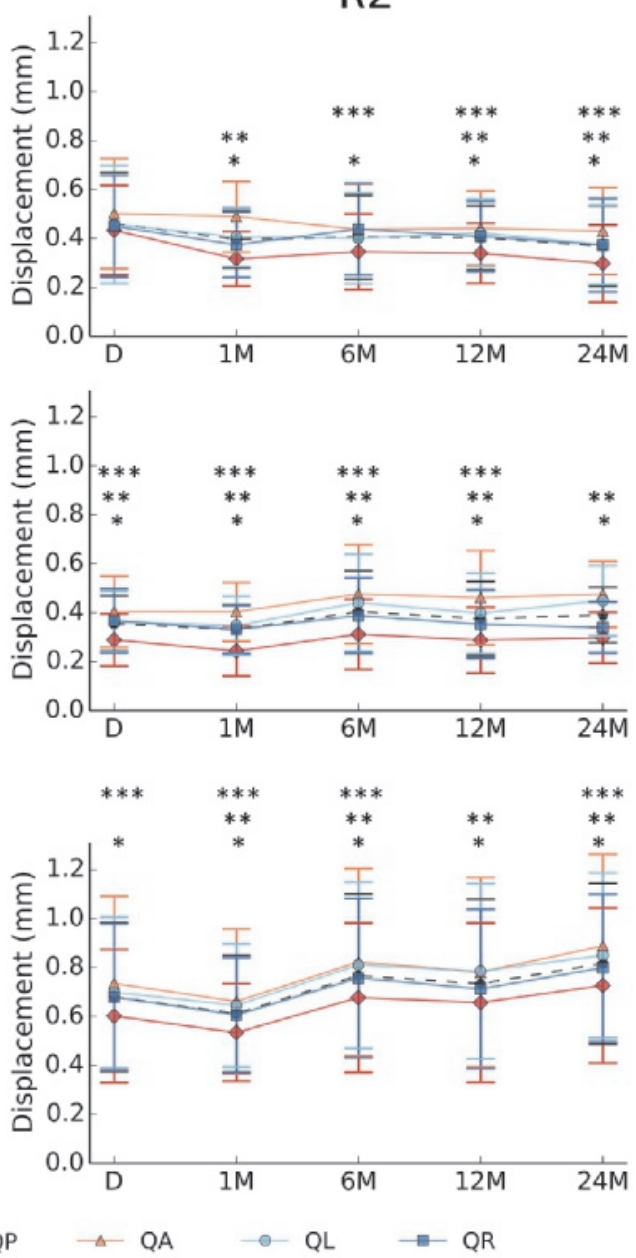

Figure 2. Mean displacement in $\mathrm{x}-, \mathrm{y}-$, and $\mathrm{z}$-direction for the full stent-rings and per stentring quadrant during follow-up. D, discharge; $\mathrm{M}$, months after EVAR; R1, first stent-ring; R2, second stent-ring; QP, posterior quadrant; QA, anterior quadrant; QL, left quadrant; $\mathrm{QR}$, right quadrant; *Displacement differed significantly between QP and QA; **Displacement differed significantly between $\mathrm{QP}$ and QL; *** Displacement differed significantly between QP and QR. 
R1
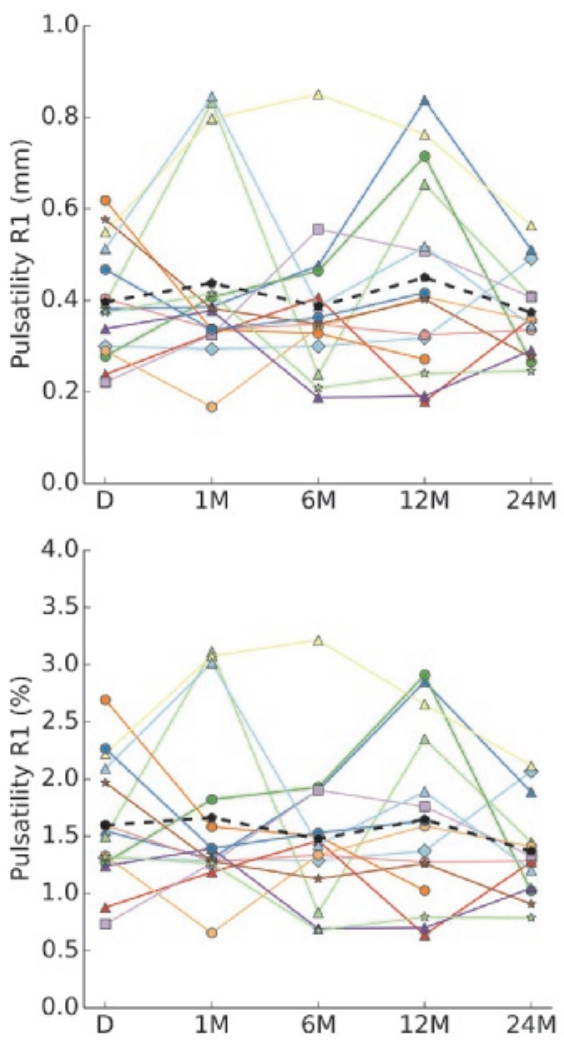

$\mathrm{R} 2$
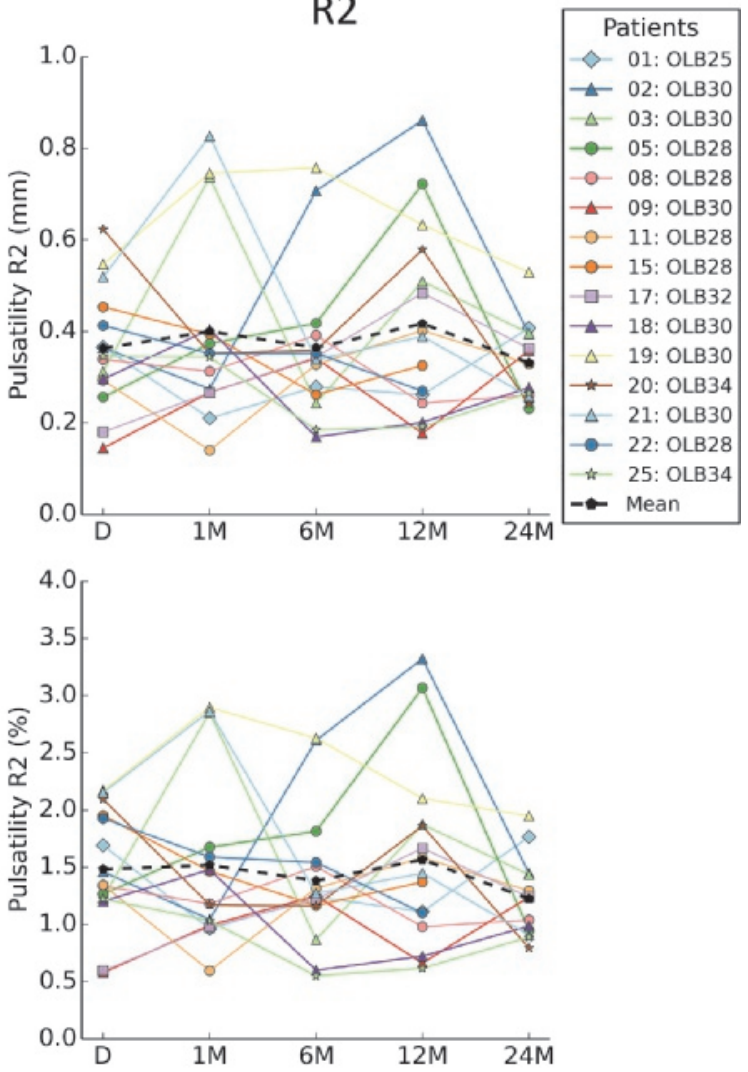

Figure 3. Maximum pulsatility of the first (left) and second (right) stent-ring during followup. D, discharge; M, months after EVAR; OLB, main body device size; R1, first stent-ring; $\mathrm{R} 2$, second stent-ring.

anterior-right posterior (LARP) direction vs. RALP-direction. Both ratios did not significantly change between time points during follow-up ( $\mathrm{p}>0.127)$. Overall, the median AP/LR and LARP/RALP ratios were $1.4[1.2,1.7]$ and $1.6[1.3,2.1]$ on the first stent-ring and $1.4[1.2,1.9]$ and $1.6[1.3,1.9]$ on the second stent-ring.

Mean curvature of the stent-rings changed significantly during follow-up, with curvature of the first and second stent-ring decreasing from $0.893 \pm 0.115 \mathrm{~cm}^{-1}$ and $0.934 \pm 0.117 \mathrm{~cm}^{-1}$ at discharge to $0.752 \pm 0.068 \mathrm{~cm}^{-1}(\mathrm{p}<0.001)$ and $0.776 \pm 0.068$ $\mathrm{cm}^{-1}(\mathrm{p}<0.001)$ at 24 months at diastole, respectively. Maximum change and mean change in curvature during the cardiac cycle did not significantly change over time ( $\mathrm{p}>0.140)$. The maximum change in curvature during follow-up was at most 0.148 $\mathrm{cm}^{-1}(6 \%)$ for the first stent-ring and $0.218 \mathrm{~cm}^{-1}(8 \%)$ for the second stent-ring. The mean change in curvature during follow-up was at most $0.048 \mathrm{~cm}^{-1}(5 \%)$ for the first stent-ring and $0.053 \mathrm{~cm}^{-1}(5 \%)$ for the second stent-ring. For the first stentring, the mean change in curvature at the anterior quadrant was significantly greater compared to the posterior quadrant at all time points $(\mathrm{p}<0.004)$ except at discharge 

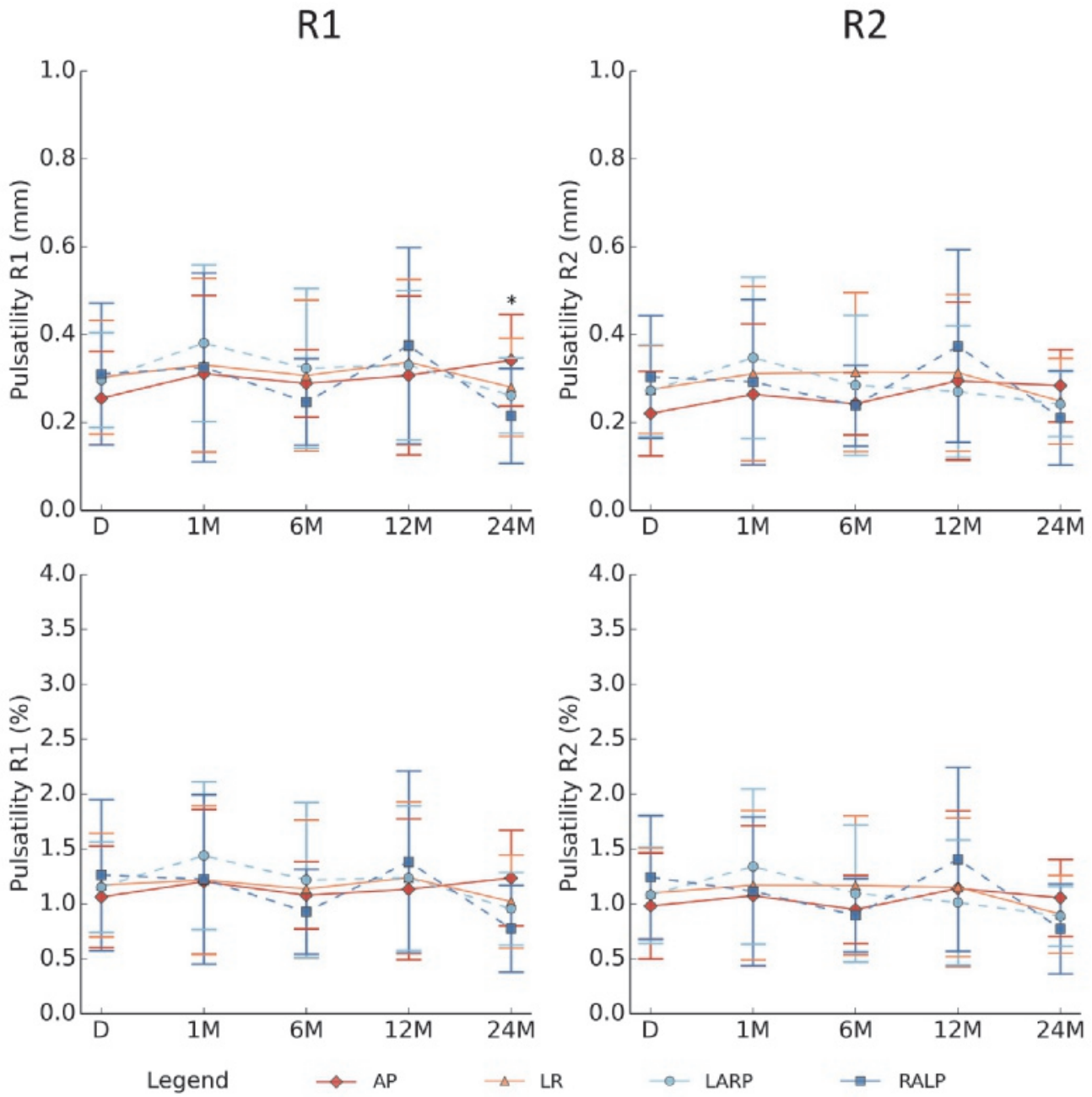

Figure 4. Pulsatility measurements during follow-up from peak-to-peak and valley-to-valley in the anterior-posterior (AP) direction or left-right (LR) direction, and from mid-to-mid in oblique direction from left anterior to right posterior (LARP) and from right anterior to left posterior (RALP) as indicated in Figure 1. D, discharge; M, months after EVAR; R1, first stent-ring; R2, second stent-ring; *Pulsatility differed significantly between the AP- and the RALP-direction. 
Table 1. Results of pulsatility and compliance over time. ${ }^{a}[$ Part I]

\begin{tabular}{|c|c|c|c|c|c|}
\hline & $\begin{array}{l}\text { Discharge } \\
(\mathrm{n}=15)\end{array}$ & $\begin{array}{l}1 \text { Month } \\
\mathrm{n}=15)\end{array}$ & $\begin{array}{l}6 \text { Months } \\
(\mathrm{n}=15)\end{array}$ & $\begin{array}{l}12 \text { Months } \\
(\mathrm{n}=15)\end{array}$ & $\begin{array}{l}24 \text { Months } \\
(\mathrm{n}=13)\end{array}$ \\
\hline $\begin{array}{l}\text { Systolic } \\
\text { pressureb, } \\
\text { mmHg } \\
\text { Diastolic } \\
\text { pressureb, } \\
\mathrm{mmHg}\end{array}$ & $\begin{array}{l}140.9 \pm 17.2 \\
(104.0-169.0) \\
- \\
76.9 \pm 10.5 \\
(64.0-96.0) \\
-\end{array}$ & $\begin{array}{l}143.5 \pm 21.4 \\
(114.0-182.0) \\
p>0.99 \\
83.1 \pm 10.4 \\
(68.0-100.0) \\
p=0.192\end{array}$ & $\begin{array}{l}151.3 \pm 17.3 \\
128.0-199.0) \\
p>0.99 \\
83.9 \pm 9.9 \\
(70.0-104.0) \\
p>0.99\end{array}$ & $\begin{array}{l}155.2 \pm 16.2 \\
(122.0-188.0) \\
p=0.272 \\
87.7 \pm 12.0 \\
(69.0-104.0) \\
p=0.035\end{array}$ & $\begin{array}{l}158.4 \pm 20.9 \\
(115.0-191.0) \\
\mathrm{p}=0.106 \\
85.8 \pm 11.1 \\
(68.0-101.0) \\
\mathrm{p}=0.263\end{array}$ \\
\hline \multicolumn{6}{|l|}{$\mathrm{R} 1$} \\
\hline $\begin{array}{l}\text { Maximum } \\
\text { pulsatility }^{c}, \%\end{array}$ & $\begin{array}{l}1.6 \pm 0.5 \\
(0.7-2.7) \\
-\end{array}$ & $\begin{array}{l}1.7 \pm 0.7 \\
(0.7-3.1) \\
p>0.99\end{array}$ & $\begin{array}{l}1.5 \pm 0.6 \\
(0.7-3.2) \\
p>0.99\end{array}$ & $\begin{array}{l}1.6 \pm 0.7 \\
(0.6-2.9) \\
p>0.99\end{array}$ & $\begin{array}{l}1.4 \pm 0.4 \\
(0.8-2.1) \\
p>0.99\end{array}$ \\
\hline $\begin{array}{l}\text { Mean } \\
\text { pulsatility }^{c}, \%\end{array}$ & $\begin{array}{l}1.2 \pm 0.4 \\
(0.6-1.9) \\
-\end{array}$ & $\begin{array}{l}1.3 \pm 0.6 \\
(0.6-2.8) \\
p>0.99\end{array}$ & $\begin{array}{c}1.1 \pm 0.4 \\
(0.4-2.0) \\
\mathrm{p}>0.99\end{array}$ & $\begin{array}{l}1.2 \pm 0.6 \\
(0.5-2.7) \\
p>0.99\end{array}$ & $\begin{array}{l}1.0 \pm 0.3 \\
(0.6-1.6) \\
p>0.99\end{array}$ \\
\hline $\begin{array}{l}\text { Asymmetry } \\
\text { ratio } \\
\text { pulsatility } \\
\text { AP/LR }\end{array}$ & $\begin{array}{l}1.6 \pm 0.5 \\
(1.1-2.7) \\
1.4 \\
{[1.2,2.0]} \\
-\end{array}$ & $\begin{array}{l}1.4 \pm 0.4 \\
(1.0-2.6) \\
1.3 \\
{[1.1,1.6]} \\
p=0.389\end{array}$ & $\begin{array}{l}1.6 \pm 0.7 \\
(1.0-3.6) \\
1.3 \\
{[1.1,1.9]} \\
p=0.890\end{array}$ & $\begin{array}{l}1.5 \pm 0.6 \\
(1.1-3.5) \\
1.3 \\
{[1.2,1.7]} \\
p=0.489\end{array}$ & $\begin{array}{l}1.5 \pm 0.4 \\
(1.1-2.6) \\
1.5 \\
{[1.2,1.7]} \\
\mathrm{p}=0.376\end{array}$ \\
\hline $\begin{array}{l}\text { Asymmetry } \\
\text { ratio } \\
\text { pulsatility } \\
\text { LARP/RALP }\end{array}$ & $\begin{array}{l}1.8 \pm 0.4 \\
(1.3-3.0) \\
1.6 \\
{[1.5,1.9]} \\
-\end{array}$ & $\begin{array}{l}1.6 \pm 0.5 \\
(1.1-2.5) \\
1.5 \\
{[1.2,2.2]} \\
p=0.389\end{array}$ & $\begin{array}{l}1.8 \pm 0.8 \\
(1.0-3.4) \\
1.6 \\
{[1.2,2.8]} \\
p=0.934\end{array}$ & $\begin{array}{l}1.7 \pm 0.7 \\
(1.1-3.6) \\
1.5 \\
{[1.2,2.3]} \\
p=0.847\end{array}$ & $\begin{array}{l}1.7 \pm 0.6 \\
(1.1-2.9) \\
1.7 \\
{[1.2,2.2]} \\
p>0.99\end{array}$ \\
\hline $\begin{array}{l}\text { Maximum } \\
\text { compliance }^{b, d} \text {, } \\
\% \\
\text { Mean } \\
\text { compliance }^{b, d} \text {, } \\
\%\end{array}$ & $\begin{array}{l}2.5 \pm 0.7 \\
(1.1-3.9) \\
- \\
1.8 \pm 0.5 \\
(0.9-2.7) \\
-\end{array}$ & $\begin{array}{l}3.0 \pm 1.9 \\
(1.1-8.9) \\
\mathrm{p}>0.99 \\
2.3 \pm 1.7 \\
(1.0-8.1) \\
\mathrm{p}>0.99\end{array}$ & $\begin{array}{l}2.2 \pm 0.8 \\
(1.0-3.8) \\
\mathrm{p}>0.99 \\
1.6 \pm 0.6 \\
(0.6-2.8) \\
\mathrm{p}>0.99\end{array}$ & $\begin{array}{l}2.5 \pm 1.2 \\
(1.0-5.0) \\
\mathrm{p}>0.99 \\
1.9 \pm 0.9 \\
(0.7-3.5) \\
\mathrm{p}>0.99\end{array}$ & $\begin{array}{l}2.1 \pm 1.1 \\
(1.2-5.3) \\
\mathrm{p}>0.99 \\
1.5 \pm 0.8 \\
(0.9-3.7) \\
\mathrm{p}>0.99\end{array}$ \\
\hline
\end{tabular}

$(\mathrm{p}=0.112)$ and greater compared to the left quadrant at 6 months $(\mathrm{p}=0.003)$ and the right quadrant at 12 months $(\mathrm{p}<0.001)$ (Figure 5). At the second stent-ring, only at 24 months the mean change in curvature at the anterior quadrant was significantly greater compared to the posterior $(\mathrm{p}<0.001)$ and right quadrant $(\mathrm{p}=0.025)$.

\section{Discussion}

The present study evaluated the cyclic adaptations of the Anaconda proximal sealing stent-rings resulting from pulsatile blood flow. This work represents our first effort to characterize complex 3D stent-graft dynamics during follow-up. Our ECG-gated CT assessment, using automated image registration techniques, demonstrated minimal pulsatile expansion and contraction $(<1 \mathrm{~mm},<3.5 \%)$ and synchronized movement of the sealing rings as seen by $<0.4 \mathrm{~mm}$ change in distance between the first and second stent-ring during the cardiac cycle. Displacement was $<0.9 \mathrm{~mm}$ in $\mathrm{x}$ - and y-direction and $<1.6 \mathrm{~mm}$ in z-direction and frequently significantly smaller at the 
Table 1. Results of pulsatility and compliance over time. ${ }^{a}[$ Part II $]$

\begin{tabular}{|c|c|c|c|c|c|}
\hline & $\begin{array}{l}\text { Discharge } \\
(\mathrm{n}=15)\end{array}$ & $\begin{array}{l}1 \text { Month } \\
\mathrm{n}=15)\end{array}$ & $\begin{array}{l}6 \text { Months } \\
(\mathrm{n}=15)\end{array}$ & $\begin{array}{l}12 \text { Months } \\
(\mathrm{n}=15)\end{array}$ & $\begin{array}{l}24 \text { Months } \\
(\mathrm{n}=13)\end{array}$ \\
\hline \multicolumn{6}{|l|}{$\mathrm{R} 2$} \\
\hline $\begin{array}{l}\text { Maximum } \\
\text { pulsatility }^{c}, \%\end{array}$ & $\begin{array}{l}1.5 \pm 0.5 \\
(0.6-2.2) \\
-\end{array}$ & $\begin{array}{l}1.5 \pm 0.7 \\
(0.6-2.9) \\
p>0.99\end{array}$ & $\begin{array}{l}1.4 \pm 0.6 \\
(0.6-2.6) \\
p>0.99\end{array}$ & $\begin{array}{l}1.6 \pm 0.8 \\
(0.6-3.3) \\
p>0.99\end{array}$ & $\begin{array}{l}1.2 \pm 0.3 \\
(0.8-1.9) \\
p>0.99\end{array}$ \\
\hline $\begin{array}{l}\text { Mean } \\
\text { pulsatility }^{c}, \%\end{array}$ & $\begin{array}{l}1.1 \pm 0.3 \\
(0.5-1.7) \\
-\end{array}$ & $\begin{array}{l}1.2 \pm 0.6 \\
(0.5-2.5) \\
p>0.99\end{array}$ & $\begin{array}{l}1.0 \pm 0.4 \\
(0.4-1.9) \\
p>0.99\end{array}$ & $\begin{array}{l}1.2 \pm 0.6 \\
(0.5-2.9) \\
p>0.99\end{array}$ & $\begin{array}{l}0.9 \pm 0.3 \\
(0.6-1.5) \\
p>0.99\end{array}$ \\
\hline $\begin{array}{l}\text { Asymmetry } \\
\text { ratio }\end{array}$ & $\begin{array}{l}1.8 \pm 0.7 \\
(1.0-3.3)\end{array}$ & $\begin{array}{l}1.5 \pm 0.4 \\
(1.0-2.4)\end{array}$ & $\begin{array}{l}1.5 \pm 0.7 \\
(1.0-3.7)\end{array}$ & $\begin{array}{l}1.8 \pm 0.9 \\
(1.1-3.6)\end{array}$ & $\begin{array}{l}1.5 \pm 0.4 \\
(1.0-2.1)\end{array}$ \\
\hline pulsatility & 1.7 & 1.4 & 1.3 & 1.3 & 1.5 \\
\hline $\mathrm{AP} / \mathrm{LR}$ & {$[1.2,2.4]$} & $\begin{array}{l}{[1.2,1.7]} \\
\mathrm{p}=0.330\end{array}$ & $\begin{array}{l}{[1.1,1.8]} \\
\mathrm{p}=0.330\end{array}$ & $\begin{array}{l}{[1.1,2.5]} \\
\mathrm{p}=0.639\end{array}$ & $\begin{array}{l}{[1.2,1.8]} \\
\mathrm{p}=0.127\end{array}$ \\
\hline $\begin{array}{l}\text { Asymmetry } \\
\text { ratio } \\
\text { pulsatility } \\
\text { LARP/RALP }\end{array}$ & $\begin{array}{l}1.7 \pm 0.6 \\
(1.0-3.6) \\
1.6 \\
{[1.2,1.9]} \\
-\end{array}$ & $\begin{array}{l}1.7 \pm 0.6 \\
(1.0-3.0) \\
1.5 \\
{[1.1,2.3]} \\
p=0.890\end{array}$ & $\begin{array}{l}1.9 \pm 1.0 \\
(1.0-4.7) \\
1.5 \\
{[1.3,1.9]} \\
p=0.978\end{array}$ & $\begin{array}{l}1.8 \pm 0.8 \\
(1.0-3.5) \\
1.6 \\
{[1.2,2.1]} \\
\mathrm{p}=0.679\end{array}$ & $\begin{array}{l}1.7 \pm 0.4 \\
(1.1-2.3) \\
1.9 \\
{[1.3,2.0]} \\
p=0.414\end{array}$ \\
\hline $\begin{array}{l}\text { Maximum } \\
\text { compliance }^{b, d}, \\
\% \\
\text { Mean } \\
\text { compliance }^{b, d}, \\
\%\end{array}$ & $\begin{array}{l}2.3 \pm 0.9 \\
(0.9-4.2) \\
- \\
1.7 \pm 0.6 \\
(0.8-3.1) \\
-\end{array}$ & $\begin{array}{l}2.7 \pm 1.7 \\
(1.0-8.1) \\
p>0.99 \\
2.1 \pm 1.5 \\
(0.9-7.2) \\
p>0.99\end{array}$ & $\begin{array}{l}2.0 \pm 0.8 \\
(0.8-3.4) \\
p>0.99 \\
1.5 \pm 0.5 \\
(0.6-2.5) \\
p>0.99\end{array}$ & $\begin{array}{l}2.3 \pm 1.2 \\
(1.0-5.3) \\
\mathrm{p}>0.99 \\
1.8 \pm 0.9 \\
(0.7-3.7) \\
\mathrm{p}>0.99\end{array}$ & $\begin{array}{l}1.9 \pm 0.9 \\
(0.9-4.5) \\
p>0.99 \\
1.4 \pm 0.7 \\
(0.8-3.4) \\
p=0.661\end{array}$ \\
\hline
\end{tabular}

Abbreviations: R1, first stent-ring; R2, second stent-ring; AP, anterior-posterior direction; LR, left-right direction; LARP, left anterior-right posterior direction; RALP, right anterior-left posterior direction.

${ }^{a}$ Data are presented as mean \pm standard deviation and range. For nonparametric data, the median [interquartile range Q1, Q3] is also presented.

${ }^{b}$ Number of patients with blood pressure measurements available: discharge, $\mathrm{n}=14$; 1 month, $\mathrm{n}=15 ; 6$ months, $\mathrm{n}=14 ; 12$ months, $\mathrm{n}=15 ; 24$ months, $\mathrm{n}=13$.

${ }^{c}$ Pulsatility is defined as the change in diametric distance (D) over the cardiac cycle (expansion and contraction), i.e., $\mathrm{D}_{\text {maximum }}-\mathrm{D}_{\text {minimum }}$. The percentage pulsatility corresponds to the percentage change in diametric distance, i.e., $100 \times\left(\mathrm{D}_{\text {maximum }}\right.$ $\left.\mathrm{D}_{\text {minimum }}\right) / \mathrm{D}_{\text {minimum }}$.

${ }^{d}$ Compliance is defined as the percentage change in diametric distance per $100 \mathrm{mmHg}$, i.e., $\left(100 \times\left(\mathrm{D}_{\text {maximum }}-\mathrm{D}_{\text {minimum }}\right) / \mathrm{D}_{\text {minimum }}\right) \times\left(100 /\left(\mathrm{BP}_{\text {systolic }}-\mathrm{BP}_{\text {diastolic }}\right)\right)$. $\mathrm{BP}$, blood pressure in $\mathrm{mmHg}$.

posterior quadrant of the stent-rings compared to other quadrants; mostly measuring greatest at the anterior quadrant. Similarly, the mean change in curvature was significantly greater at the anterior quadrant compared to the other quadrants at multiple time points and reached a value of at most $7 \%$ at the anterior quadrant. No significant cardiac pulsatility-induced changes were observed between time points during 2 years of follow-up. Instead, after implantation immediate low pulsatility values were observed from discharge on.

Quantifying the cyclic adaptation of stent-grafts is imperative for the development, design verification and further improvement of stent-grafts, yet the extent of 
Table 2. Results of stent-ring curvature during the cardiac cycle.

\begin{tabular}{|c|c|c|c|c|c|}
\hline & $\begin{array}{l}\text { Discharge } \\
(\mathrm{n}=15)\end{array}$ & $\begin{array}{l}1 \text { Month } \\
(\mathrm{n}=15)\end{array}$ & $\begin{array}{l}6 \text { Months } \\
(\mathrm{n}=15)\end{array}$ & $\begin{array}{l}12 \text { Months } \\
(\mathrm{n}=15)\end{array}$ & $\begin{array}{l}24 \text { Months } \\
(\mathrm{n}=13)\end{array}$ \\
\hline \multicolumn{6}{|l|}{$\mathrm{R} 1$} \\
\hline \multicolumn{6}{|l|}{$\begin{array}{l}\text { Mean } \\
\text { curvature, } \\
\text { cm-1 }\end{array}$} \\
\hline Minimum $^{a}$ & $\begin{array}{l}0.884 \pm 0.113 \\
(0.684-1.082) \\
-\end{array}$ & $\begin{array}{l}0.824 \pm 0.102 \\
(0.669-1.057) \\
p=0.006\end{array}$ & $\begin{array}{l}0.778 \pm 0.077 \\
(0.642-0.921) \\
p<0.001\end{array}$ & $\begin{array}{l}0.770 \pm 0.069 \\
(0.633-0.893) \\
p<0.001\end{array}$ & $\begin{array}{l}0.748 \pm 0.067 \\
(0.633-0.882) \\
p<0.001\end{array}$ \\
\hline Maximum $^{b}$ & $\begin{array}{l}0.893 \pm 0.115 \\
(0.689-1.095) \\
-\end{array}$ & $\begin{array}{l}0.832 \pm 0.103 \\
(0.674-1.071) \\
p=0.009\end{array}$ & $\begin{array}{l}0.785 \pm 0.078 \\
(0.644-0.932) \\
p<0.001\end{array}$ & $\begin{array}{l}0.778 \pm 0.070 \\
(0.637-0.900) \\
p<0.001\end{array}$ & $\begin{array}{l}0.752 \pm 0.068 \\
(0.636-0.890) \\
\mathrm{p}<0.001\end{array}$ \\
\hline \multicolumn{6}{|l|}{$\begin{array}{l}\text { Change in } \\
\text { curvature }^{c} \text {, } \\
\text { cm-1 }\end{array}$} \\
\hline $\begin{array}{l}\text { Maximum } \\
\text { change }\end{array}$ & $\begin{array}{l}0.071 \pm 0.033 \\
(0.037-0.148) \\
-\end{array}$ & $\begin{array}{l}0.053 \pm 0.019 \\
(0.034-0.101) \\
p=0.672\end{array}$ & $\begin{array}{l}0.054 \pm 0.020 \\
(0.017-0.096) \\
p>0.99\end{array}$ & $\begin{array}{l}0.057 \pm 0.029 \\
(0.021-0.142) \\
p>0.99\end{array}$ & $\begin{array}{l}0.053 \pm 0.018 \\
(0.029-0.087) \\
p>0.99\end{array}$ \\
\hline $\begin{array}{l}\text { Mean } \\
\text { change }\end{array}$ & $\begin{array}{l}0.029 \pm 0.009 \\
(0.017-0.048) \\
-\end{array}$ & $\begin{array}{l}0.025 \pm 0.005 \\
(0.018-0.034) \\
p>0.99\end{array}$ & $\begin{array}{l}0.026 \pm 0.007 \\
(0.010-0.039) \\
p>0.99\end{array}$ & $\begin{array}{l}0.025 \pm 0.008 \\
(0.012-0.035) \\
\mathrm{p}>0.99\end{array}$ & $\begin{array}{l}0.023 \pm 0.005 \\
(0.015-0.035) \\
p>0.99\end{array}$ \\
\hline \multicolumn{6}{|l|}{$\mathrm{R} 2$} \\
\hline \multicolumn{6}{|l|}{$\begin{array}{l}\text { Mean } \\
\text { curvature, } \\
\text { cm-1 }\end{array}$} \\
\hline Minimum $^{a}$ & $\begin{array}{l}0.926 \pm 0.115 \\
(0.778-1.159)-\end{array}$ & $\begin{array}{l}0.868 \pm 0.106 \\
(0.746-1.091) \\
p=0.004\end{array}$ & $\begin{array}{l}0.813 \pm 0.086 \\
(0.702-0.998) \\
p<0.001\end{array}$ & $\begin{array}{l}0.805 \pm 0.070 \\
(0.698-0.926) \\
p<0.001\end{array}$ & $\begin{array}{l}0.771 \pm 0.067 \\
(0.674-0.904) \\
p<0.001\end{array}$ \\
\hline Maximum $^{b}$ & $\begin{array}{l}0.934 \pm 0.117 \\
(0.786-1.174)-\end{array}$ & $\begin{array}{l}0.877 \pm 0.106 \\
(0.748-1.101) \\
\mathrm{p}=0.005\end{array}$ & $\begin{array}{l}0.820 \pm 0.087 \\
(0.707-1.007) \\
p<0.001\end{array}$ & $\begin{array}{l}0.813 \pm 0.071 \\
(0.707-0.932) \\
\mathrm{p}<0.001\end{array}$ & $\begin{array}{l}0.776 \pm 0.068 \\
(0.676-0.911) \\
\mathrm{p}<0.001\end{array}$ \\
\hline \multicolumn{6}{|l|}{$\begin{array}{l}\text { Change in } \\
\text { curvature }^{c} \text {, } \\
\text { cm-1 }\end{array}$} \\
\hline $\begin{array}{l}\text { Maximum } \\
\text { change }\end{array}$ & $\begin{array}{l}0.072 \pm 0.045 \\
(0.031-0.218) \\
-\end{array}$ & $\begin{array}{l}0.058 \pm 0.029 \\
(0.030-0.134) \\
p>0.99\end{array}$ & $\begin{array}{l}0.061 \pm 0.037 \\
(0.018-0.166) \\
p>0.99\end{array}$ & $\begin{array}{l}0.070 \pm 0.054 \\
(0.023-0.239) \\
p>0.99\end{array}$ & $\begin{array}{l}0.051 \pm 0.017 \\
(0.030-0.080) \\
p>0.99\end{array}$ \\
\hline $\begin{array}{l}\text { Mean } \\
\text { change }\end{array}$ & $\begin{array}{l}0.028 \pm 0.009 \\
(0.016-0.053) \\
-\end{array}$ & $\begin{array}{l}0.026 \pm 0.007 \\
(0.016-0.038) \\
p>0.99\end{array}$ & $\begin{array}{l}0.025 \pm 0.008 \\
(0.010-0.040) \\
p>0.99\end{array}$ & $\begin{array}{l}0.024 \pm 0.007 \\
(0.013-0.036) \\
p>0.99\end{array}$ & $\begin{array}{l}0.022 \pm 0.005 \\
(0.015-0.033) \\
p=0.140\end{array}$ \\
\hline
\end{tabular}

Abbreviations: R1, first stent-ring; R2, second stent-ring.

${ }^{a}$ Representing the mean peak-systolic curvature.

${ }^{b}$ Representing the mean end-diastolic curvature.

${ }^{c}$ Change during the cardiac cycle. 


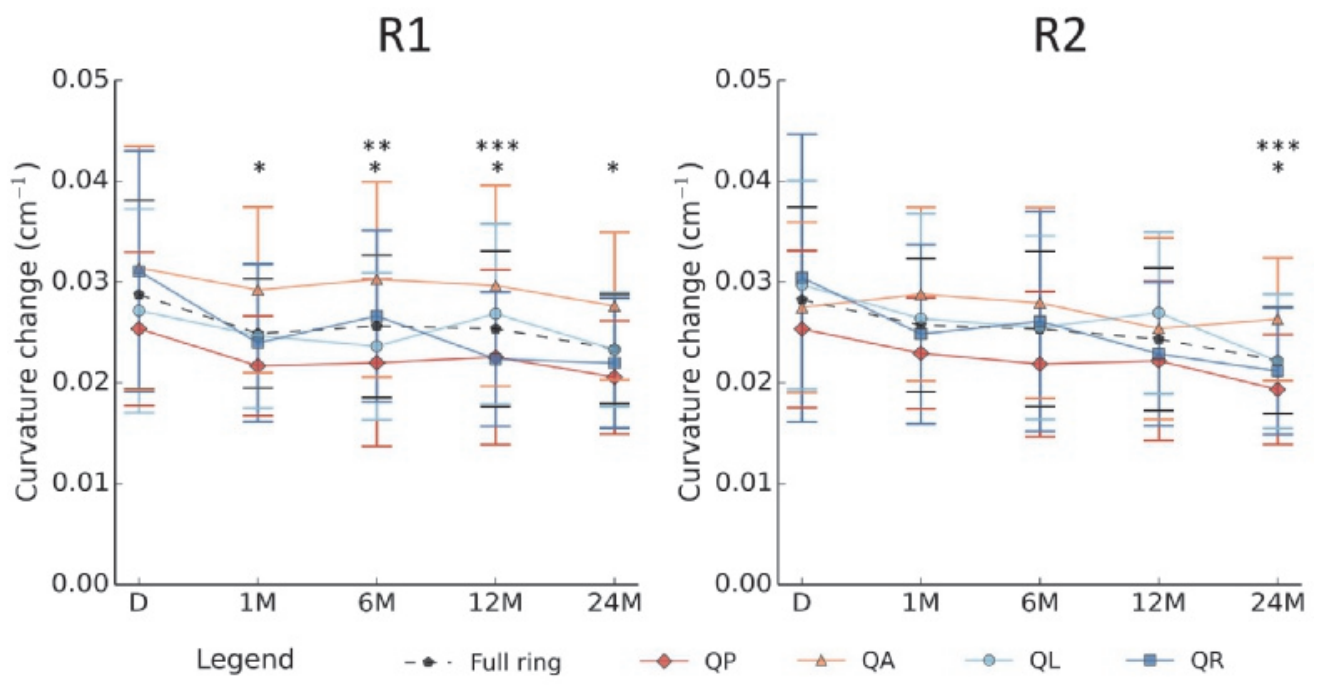

Figure 5. Mean change in curvature for the full stent-rings and per stent-ring quadrant during follow-up. D, discharge; M, months after EVAR; R1, first stent-ring; R2, second stent-ring; QP, posterior quadrant; QA, anterior quadrant; QL, left quadrant; QR, right quadrant; *Curvature change during the cardiac cycle differed significantly between QP and QA; **Curvature change during the cardiac cycle differed significantly between QL and QA; ***Curvature change during the cardiac cycle differed significantly between QR and QA.

cyclic adaptation in situ is largely unknown[1,24,25]. While several studies reported useful insights regarding abdominal aortic distension[24,26-28], albeit limited to 2D planes and lacking a validation of accuracy, we found only two groups that quantified $3 \mathrm{D}$ dynamics of the stent-graft skeleton itself[25,29,30]. Koning et al. underlined the importance to quantify 3D stent-graft dynamics and demonstrated the feasibility of fluoroscopic Roentgenographic stereophotogrammetric analysis (FRSA) to study $3 \mathrm{D}$ stent-graft dynamics in situ[25]. However useful for clinical application considering radiation exposure, this methodology is so far dependent on identifiable markers on the stent-graft (positioning markers, welding points) and does not enable motion analysis of the full wire frame structure. Singland et al. studied the dynamics of a homemade aorto-uni-iliac endograft in 11 patients using ECG-gated CT at a mean follow-up of 4.3 years by manually selecting the eyelets that connect the stent struts in 2D planes to subsequently model a 3D stent-graft structure[29]. A disadvantage of their methodology is the simplification of the complexity and morphological variability of the stent-graft structure. Moreover, manual selection of the eyelets induced a considerable margin of error of at least one pixel $(0.36-0.50 \mathrm{~mm})$. In addition, this methodology may not be applicable to stent-grafts with circular stents or M-shaped stents that comprise curved structures. Nevertheless, this study provided useful insights for the stent-graft studied and was able to visualize areas with the greatest stress by computing angular variation between eyelet triangles, i.e., modeled stent struts. This study reported a mean radial distension of $0.6 \pm 0.4 \mathrm{~mm}(6.2 \pm 2.4 \%)$ for 7 patients while 3 patients showed radial distension and/or angular variation of 
$>1 \mathrm{~mm}(6.6 \%)$ up to $1.5 \mathrm{~mm}(8.1 \%)$ and $>6^{\circ}$ up to $7.1^{\circ}$, respectively. Our present study measured pulsatile distension $<1 \mathrm{~mm}(3.5 \%)$ in all patients. Considering that commercially available fatigue test banks apply pulsation at a 'worst case' $5 \%$ of the device diameter[1,29], our present study indicates that the proximal stent-rings adequately counteract radial vessel expansion and contraction to prevent stent fatigue. Furthermore, limited pulsatile distention seems favorable to maintain a functional seal and fixation, especially since greater preoperative pulsatile distention has been associated with stent-graft migration[31]. Still, damping of aortic distension by the implanted stent-graft may affect the physiologic haemodynamic flow and possibly increases the risk of atherosclerotic events[22].

We observed minimal changes in curvature of the stent-rings during the cardiac cycle, which suggests a low risk of stent fracture by metal fatigue. These observations are in line with literature, as no cases of fracture of Anaconda sealing rings in the treatment of infrarenal AAA have been reported. Still, fracture of a proximal stentring in a fenestrated Anaconda device 3 years after treatment of a juxtarenal AAA has recently been observed, though with prosperous aneurysm shrinkage[32]. Investigation of this fracture case by Terumo Aortic identified post-implant disease progression as well as calcification in the vicinity of the stent. These, together with a greater degree of stent-ring motion for fenestrated Anaconda compared with the infrarenal Anaconda (postulated from observations of fenestrated Anaconda pulsatility[21]), may have caused unusually high localized loading of the stent-ring for this particular case. It should be noted that the ability to measure localized stent-ring curvature changes, as per the novel methodology described here, could provide a very useful tool for such stent fracture investigations where post-implant ECG-gated CT data is available.

The dynamic stress by bending in the present study was typically at its maximum at the anterior, left, or right quadrant but never at the posterior quadrant, as shown in the case example presented (Figure 6). Similarly, displacement was often greater in the anterior, left, or right quadrant compared to the posterior quadrant, indicating anisotropic deformation. It has previously been observed that the aortic neck expands asymmetrically during the cardiac cycle[24,27,33]. Two of these studies reported postoperative asymmetry ratios of radius change over the major vs. the minor axis ranging from 1.08 to 2.01 at $1 \mathrm{~cm}$ below the lowest renal artery and the direction of distension showed a tendency to left anterior[24,27]. In our present study, the asymmetry ratio for the proximal stent-rings ranged from 1.0 to 4.7 , yet no statistically significant differences were observed between perpendicular directions of distension.

The results of the present study may support further evaluation of device durability. However, the low number of patients may necessitate additional evaluation on a larger patient sample in order to extrapolate the observations to a general population. It should be considered that lower oversizing percentages compared to these applied in the present patient sample $(31 \pm 9 \%)$ may allow for greater pulsatile distension and deformation. Additionally, younger patients may have a more compliant aortic neck, although preoperative pulsatile neck distension was previously observed to be comparable between younger $(\leq 65)$ and older $(>65)$ AAA patients[34]. Moreover, to enhance clinical relevance, also Z-stent and suprarenal fixation designs should be considered. 


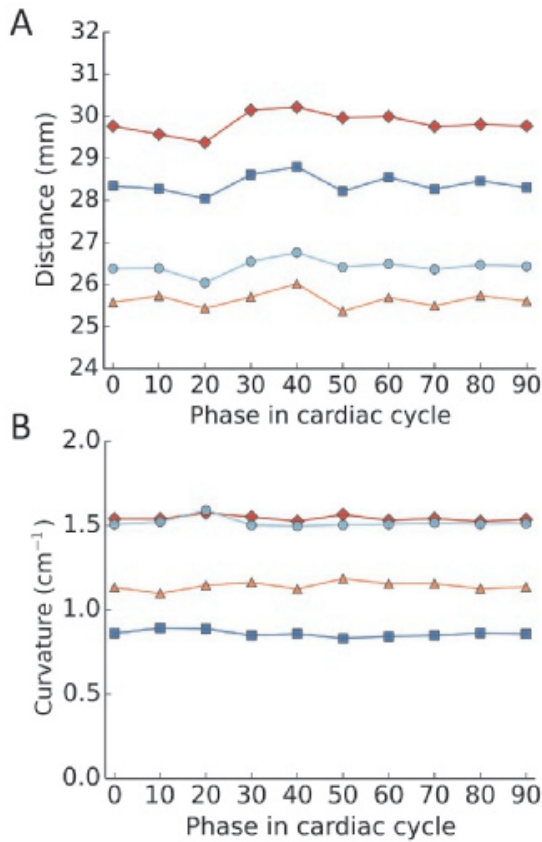

C

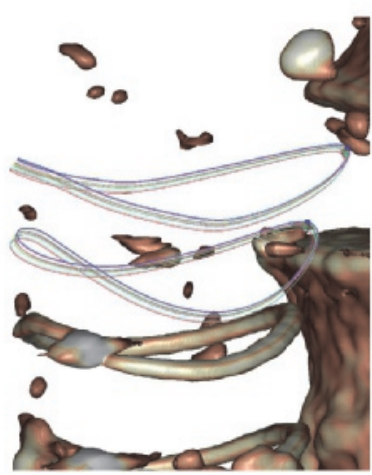

D
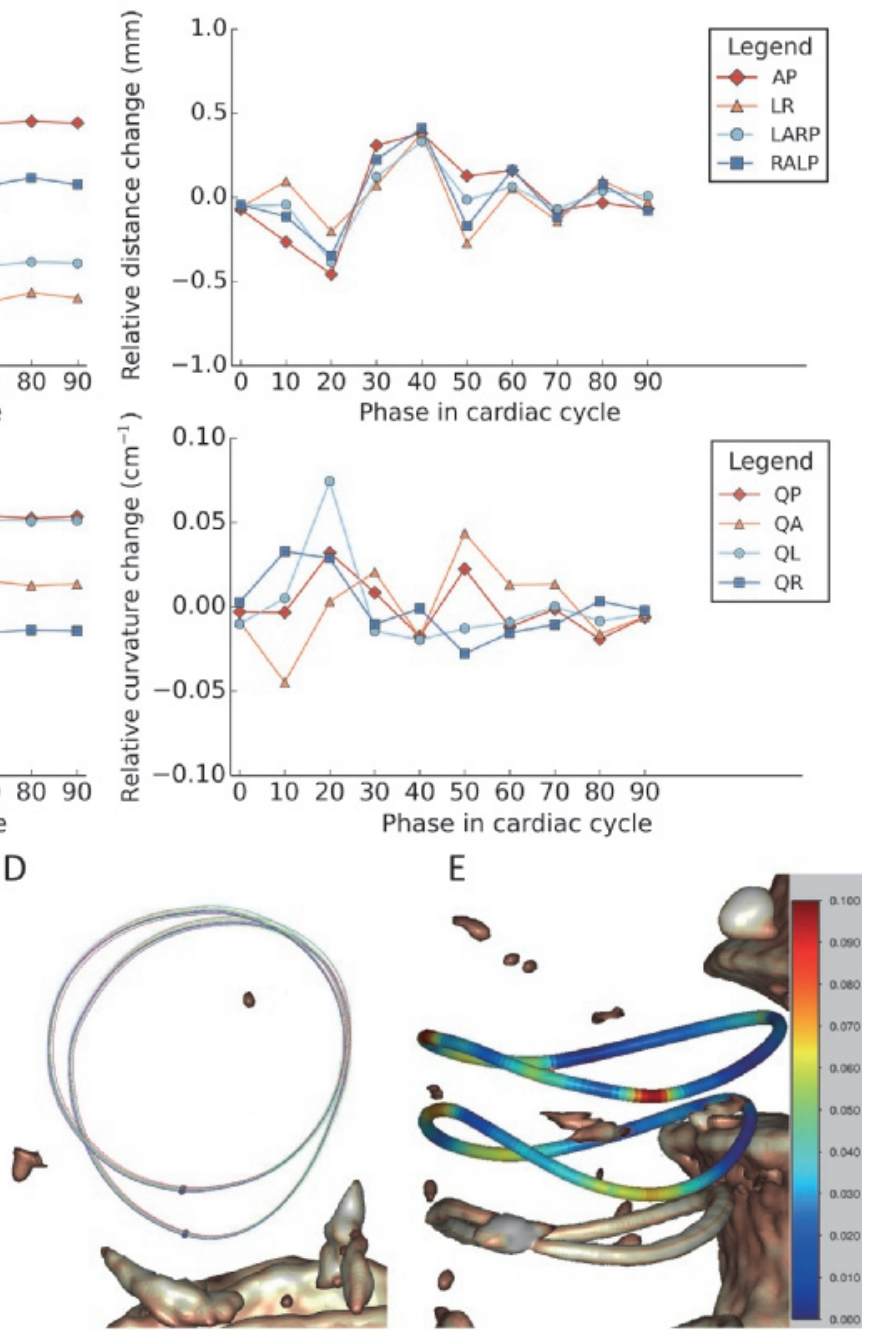

Figure 6. Case example (\#2) showing pulsatile distension and curvature change during the cardiac cycle at 12 months follow-up. Distances over the four directions of distension (Figure 1) of the first stent-ring are shown during the cardiac cycle (A, left) as well as the relative change in distance with respect to the distance at mid cardiac cycle (A, right). For each quadrant of the first-stent ring, curvature during the cardiac cycle is shown for the point with the maximum change in curvature $(\mathrm{B}$, left) as well as the relative change in curvature with respect to curvature at mid cardiac cycle $(\mathrm{B}$, right). The relative position of the stent-rings in each phase of the cardiac cycle is displayed in a $3 \mathrm{D}$ visualization in a lateral view from left to right $(\mathrm{C})$ and in a top view from superior to inferior (D). Finally, a 3D color-coded visualization of stent-ring curvature change during the cardiac cycle is shown (E). The color bar ranges from 0 to $0.1 \mathrm{~cm}^{-1}$. 


\section{Conclusion}

Using modern techniques based on 3D image segmentation and registration, we were able to visualize and quantify cardiac pulsatility-induced motion and deformation of the 3D stent-ring structure of the Anaconda sealing rings. This study found the proximal stent-rings to adequately counteract aortic pulsatility as seen by limited pulsatile distension $(<3.5 \%)$, which reduces the risk of stent material fatigue. The methodology presented in the present work enables to identify stent locations that are most susceptible to dynamic stress by cyclic bending. These regions of maximum cyclic stress are subject to reduced fatigue safety factors under in-vivo loading conditions, and are therefore of particular importance when considering device durability. This work serves as a foundation for accurate stress-strain analysis, fatigue life evaluation and design verification. Future studies, applying the present methodology to other types of stent-grafts in abdominal, thoraco-abdominal, and thoracic EVAR are expected.

\section{Acknowledgments}

The authors thank prof.dr.ir. B.J. Geurts (Chairholder for Multiscale Modeling and Simulation in the Department of Applied Mathematics at the University of Twente) for critically reviewing this chapter.

\section{Declaration of conflicting interests}

R.H. Geelkerken is consultant for Terumo Aortic.

\section{Funding}

This study was funded in part by an unrestricted research grant from Vascutek Terumo, trading as Terumo Aortic, and in part by the PPP Allowance made available by Health Holland, Top Sector Life Sciences \& Health, to stimulate public-private partnerships.

\section{References}

1. Chuter TAM. Durability of Endovascular Infrarenal Aneurysm Repair: When Does Late Failure Occur and Why? Semin Vasc Surg. 2009;22:102-10.

2. Jacobs TS, Won J, Gravereaux EC, Faries PL, Morrissey N, Teodorescu VJ, et al. Mechanical failure of prosthetic human implants: A 10-year experience with aortic stent graft devices. J Vasc Surg. 2003;37:16-26.

3. Roos JE, Hellinger JC, Hallet R, Fleischmann D, Zarins CK, Rubin GD. Detection of endograft fractures with multidetector row computed tomography. J Vasc Surg. 2005;42:1002-6.

4. Ruthrauff AA, King MW, Soulez G, Tan KT, Crawford SA, Roche-Nagle G, et al. Effects of Pulsatile Fatigue on in Situ Antegrade Fenestrated Polyester Stent Grafts Deployed in a Patient-Specific Phantom Model of Juxtarenal Aortic Aneurysm. J Vasc Interv Radiol. 2015;26:1551-8.

5. Kleinstreuer C, Li Z, Basciano CA, Seelecke S, Farber MA. Computational mechanics of Nitinol stent grafts. J Biomech. 2008;41:2370-8.

6. Demanget N, Duprey A, Badel P, Orgéas L, Avril S, Geindreau C, et al. Finite Element Analysis of the Mechanical Performances of 8 Marketed Aortic Stent-Grafts. J Endovasc Ther. 2013;20:523-35. 
7. Thomas B, Sanchez L. Proximal migration and endoleak: Impact of endograft design and deployment techniques. Semin Vasc Surg. 2009;22:201-6.

8. Morris L, Delassus P, Walsh M, McGloughlin T. A mathematical model to predict the in vivo pulsatile drag forces acting on bifurcated stent grafts used in endovascular treatment of abdominal aortic aneurysms (AAA). J Biomech. 2004;37:1087-95.

9. Freyrie A, Gargiulo M, Testi G, Faglioli G, Rossi C, Mauro R, et al. Midterm results of Anaconda $^{\mathrm{TM}}$ infrarenal aortic endografts: a single-center prospective study. Ital J Vasc Endovasc Surg. 2009;16:1-7.

10. Freyrie A, Testi G, Faggioli GL, Gargiulo M, Giovanetti F, Serra C, et al. Ring-stents supported infrarenal aortic endograft fits well in abdominal aortic aneurysms with tortuous anatomy. J Cardiovasc Surg (Torino). 2010;51:467-74.

11. Freyrie A, Gallitto E, Gargiulo M, Faggioli G, Massoni CB, Mascoli C, et al. Results of the endovascular abdominal aortic aneurysm repair using the Anaconda aortic endograft. J Vasc Surg. 2014;60:1132-9.

12. Saratzis N, Melas N, Saratzis A, Lazarides J, Ktenidis K, Tsakiliotis S, et al. Anaconda aortic stent-graft: single-center experience of a new commercially available device for abdominal aortic aneurysms. J Endovasc Ther. 2008;15:33-41.

13. Karkos CD, Kapetanios DM, Anastasiadis PT, Grigoropoulou FS, Kalogirou TE, Giagtzidis IT,

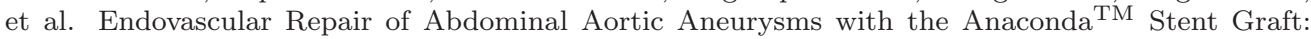
Mid-term Results from a Single Center. Cardiovasc Intervent Radiol. 2015;38:1416-24.

14. Majumder B, Urquhart G, Edwards R, Irshad K, Velu R, Reid DB. Early clinical experience with the Anaconda re-deployable endograft in 106 patients with abdominal aortic aneurism: the west of Scotland Anaconda registry. Scott Med J. 2012;57:61-4.

15. Rödel SGJ, Zeebregts CJ, Huisman AB, Geelkerken RH. Results of the Anaconda endovascular graft in abdominal aortic aneurysm with a severe angulated infrarenal neck. J Vasc Surg. 2014;59:1495-1501.e1.

16. Rödel SGJ, Geelkerken RH, Prescott RJ, Florek HJ, Kasprzak P, Brunkwall J. The Anaconda AAA stent graft system: 2-year clinical and technical results of a multicentre clinical evaluation. Eur J Vasc Endovasc Surg. 2009;38:732-40.

17. Rödel SGJ, Zeebregts CJ, Meerwaldt R, van der Palen J, Geelkerken RH. Incidence and treatment of limb occlusion of the Anaconda endograft after endovascular aneurysm repair. J Endovasc Ther. 2018;26:113-20.

18. Koenrades MA, Klein A, Leferink AM, Slump CH, Geelkerken RH. Evolution of the Proximal Sealing Rings of the Anaconda Stent-Graft After Endovascular Aneurysm Repair. J Endovasc Ther. 2018;25:480-491.

19. Klein A, Renema W, Vliet JA, Oostveen LJ, Hoogeveen Y, Schultze Kool LJ, et al. Motion Calculations on Stent Grafts in AAA. In: Grundmann RT, editor. Diagnosis, Screening and Treatment of Abdominal, Thoracoabdominal and Thoracic Aortic Aneurysms. [Rijeka]: InTechOpen; 2011. p. 125-44.

20. Klein A, van der Vliet JA, Oostveen LJ, Hoogeveen Y, Schultze Kool LJ, Renema WKJ, et al. Automatic segmentation of the wire frame of stent grafts from CT data. Med Image Anal. $2012 ; 16: 127-39$.

21. Koenrades MA, Struijs EM, Klein A, Kuipers H, Reijnen MMPJ, Slump CH, et al. Quantitative Stent Graft Motion in ECG Gated CT by Image Registration and Segmentation: In Vitro Validation and Preliminary Clinical Results. Eur J Vasc Endovasc Surg. 2019;[Epub ahead of print].

22. Oliver JJ, Webb DJ. Noninvasive Assessment of Arterial Stiffness and Risk of Atherosclerotic Events. Arterioscler Thromb Vasc Biol. 2003;23:554-66.

23. Schuurmann RCL, Kuster L, Slump CH, Vahl A, Van Den Heuvel DAF, Ouriel K, et al. Aortic curvature instead of angulation allows improved estimation of the true aorto-iliac trajectory. Eur J Vasc Endovasc Surg. 2016;51:216-24.

24. van Keulen JW, Vincken KL, van Prehn J, Tolenaar JL, Bartels LW, Viergever MA, et al. The Influence of Different Types of Stent Grafts on Aneurysm Neck Dynamics after Endovascular 
Aneurysm Repair. Eur J Vasc Endovasc Surg. 2010;39:193-9.

25. Koning OHJ, Kaptein BL, van der Vijver R, Dias N V., Malina M, Schalij MJ, et al. Fluoroscopic Roentgen stereophotogrammetric analysis (FRSA) to study three-dimensional stent graft dynamics. J Vasc Surg. 2009;50:407-12.

26. van Herwaarden JA, Muhs BE, Vincken KL, van Prehn J, Teutelink A, Bartels LW, et al. Aortic Compliance Following EVAR and the Influence of Different Endografts: Determination Using Dynamic MRA. J Endovasc Ther. 2006;13:406-14.

27. van Prehn J, van Herwaarden JA, Vincken KL, Verhagen HJM, Moll FL, Bartels LW. Asymmetric aortic expansion of the aneurysm neck: Analysis and visualization of shape changes with electrocardiogram-gated magnetic resonance imaging. J Vasc Surg. 2009;49:1395-402.

28. van Keulen JW, van Prehn J, Prokop M, Moll FL, van Herwaarden JA. Dynamics of the Aorta Before and After Endovascular Aneurysm Repair: A Systematic Review. Eur J Vasc Endovasc Surg. 2009;38:586-96.

29. Singland J-D, Mitton D, Guillaume A, Cluzel P, Goasdoue P, Lavaste F, et al. Dynamics of Homemade Aortic Endografts: In Vivo Study in Humans with Computed Tomography Scanner Modeling. Ann Vasc Surg. 2010;24:127-39.

30. Koning OHJ, Kaptein BL, Garling EH, Hinnen JW, Hamming JF, Valstar ER, et al. Assessment of three-dimensional stent-graft dynamics by using fluoroscopic roentgenographic stereophotogrammetric analysis. J Vasc Surg. 2007;46:773-9.

31. Van Keulen JW, Moll FL, Barwegen GK, Vonken EPA, Van Herwaarden JA. Pulsatile distension of the proximal aneurysm neck is larger in patients with stent graft migration. Eur J Vasc Endovasc Surg. 2010;40:326-31.

32. Tielliu I, Zeebregts C. Fractured Proximal Nitinol Ring in a Fenestrated Anaconda Device. Eur J Vasc Endovasc Surg. 2019;57:553.

33. Arko FR, Murphy EH, Davis CM, Johnson ED, Smith ST, Zarins CK. Dynamic geometry and wall thickness of the aortic neck of abdominal aortic aneurysms with intravascular ultrasonography. J Vasc Surg. 2007;46:891-7.

34. Zandvoort HJA, Moll FL, Domanian A, Van Keulen JW, Vonken EJPA, Van Herwaarden JA. Preoperative infra- and suprarenal aortic pulsatile distension is comparable between relatively young and older patients with an abdominal aortic aneurysm. Ann Vasc Surg. 2014;28:845-9. 


\section{Appendix A}

The following are the Supplementary data to this chapter:

Supplemental Video S1, S2, and S3.

Supplemental videos can be accessed online:

Video S1: https://vimeo.com/363926350

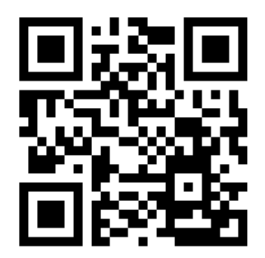

Video S2: https://vimeo.com/363930719

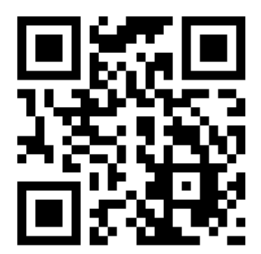

Video S3: https://vimeo.com/363932533

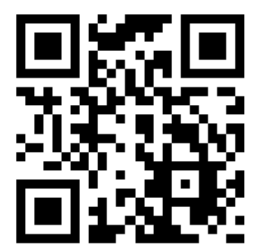





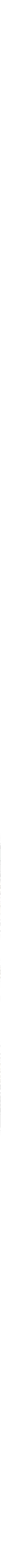





\section{Chapter 8}

\section{Dynamic computed tomography angiography analysis of cardiac pulsatility-induced motion and deformation after endovascular aneurysm sealing with chimney grafts}

Maaike A. Koenrades ${ }^{a, b}, c$, Esmé J. Donselaar ${ }^{d, e}$, Mirthe A.J.M. van $\operatorname{Erp}^{f}$, Tom G.J. Loonen ${ }^{f}$, Pim van Lochem ${ }^{f}$, Almar Klein $^{g}$, Robert H. Geelkerken ${ }^{a, b}$, Michel M.P.J. Reijnen ${ }^{a, d}$

${ }^{a}$ Multi-modality Medical Imaging (M3I) group, Faculty of Science and Technology, Technical Medical Centre, University of Twente, Enschede, the Netherlands

${ }^{b}$ Department of Vascular Surgery, Medisch Spectrum Twente, Enschede, the Netherlands

${ }^{c}$ Robotics and Mechatronics (RaM) group, Faculty of Electrical Engineering, Mathematics and Computer Science, Technical Medical Centre, University of Twente, Enschede, the Netherlands

${ }^{d}$ Department of Vascular Surgery, Rijnstate, Arnhem, the Netherlands

${ }^{e}$ Department of Anesthesiology, St. Antonius Hospital, Nieuwegein, the Netherlands

${ }^{f}$ Technical Medicine, Faculty of Science and Technology, Technical Medical Centre, University of Twente, Enschede, the Netherlands

${ }^{g}$ Independent Scholar, Enschede, the Netherlands 


\section{Abstract}

Objective: To evaluate the proximal stability of the chimney EndoVascular Aneurysm Sealing configuration (chEVAS) during the cardiac cycle by investigating the cardiac pulsatility-induced movement and deformation.

Methods: We retrospectively analyzed postoperative electrocardiogram (ECG)gated computed tomography angiography (CTA) scans of 11 chEVAS cases (9 primary chEVAS plus 2 chEVAS-in-chEVAS). ChEVAS procedures were conducted between September 2013 and June 2016. Motion and deformation of the EVAS stents, the chimney grafts and the stented branch vessels were evaluated during the cardiac cycle using an established combination of image registration and segmentation techniques.

Results: ECG-gated CTA scans of 11 chEVAS configurations including 22 EVAS stents and 20 chimney grafts were analyzed. The 3D displacement was at most 1.7 $\mathrm{mm}$ for both the EVAS stents and the chimney grafts. The maximum change in distance between components was no more than $0.4 \mathrm{~mm}$ and did not differ between EVAS-to-EVAS stent and EVAS stent-to-chimney $(0.2 \pm 0.1 \mathrm{~mm}$ vs. $0.2 \pm 0.1$ $\mathrm{mm}, \mathrm{p}=0.823)$. The mean change in chimney deflection angle was $1.2 \pm 0.7^{\circ}$; the maximum change was highest for the SMA $\left(2.6^{\circ}\right)$. The EVAS stent-to-chimney angles for the LRA, RRA and SMA varied on average by respectively $0.7 \pm 0.3^{\circ}$ $\left(0.4-1.3^{\circ}\right), 1.0 \pm 0.3^{\circ}\left(0.5-1.7^{\circ}\right)$, and $0.8 \pm 0.4^{\circ}\left(0.3-1.3^{\circ}\right)$ during the cardiac cycle. The end-stent angles for the LRA, RRA, and SMA varied on average by respectively $1.7 \pm 0.9^{\circ}\left(0.5-3.3^{\circ}\right), 1.9 \pm 0.8^{\circ}\left(0.7-3.3^{\circ}\right)$, and $1.3 \pm 0.4^{\circ}\left(0.7-1.6^{\circ}\right)$ during the cardiac cycle. Overall, the end-stent angles varied on average by $1.7 \pm 0.8^{\circ}\left(0.5-3.3^{\circ}\right)$.

Conclusion: The chEVAS configuration proved to be stable during the cardiac cycle, as demonstrated by minimal cyclical changes in distance between device components and angulation between the EVAS stents and the chimney grafts. The limited deflection angles of the chimney grafts reduce the risk of bending fatigue but the more apparent change in end-stent angle distal to the chimney graft may raise concerns regarding late branch occlusion or stenosis.

Keywords: Endovascular aneurysm sealing, chimney graft, pulsatile motion, electrocardiogram-gated computed tomography angiography, proximal sealing and fixation, stability 


\section{Introduction}

The use of chimney, or parallel, grafts combined with an endovascular aneurysm device provides a versatile alternative to fenestrated endografts for endovascular repair of para- and juxtarenal abdominal aortic aneurysms (AAA) $[1,2]$. While the chimney technique in combination with conventional endografts (chEVAR) may be troubled by chimney graft compression and gutter-endoleak formation[3], the combination with sac-sealing endografts (chEVAS) has the potential to prevent these complications $[4,5]$. The multicenter ASCEND registry demonstrated favorable results for chEVAS[4], although the data are not robust beyond 12 months of follow-up. In addition, chEVAS was recently shown to be an effective method to treat complications of previous EVAR procedures[6,7]. However, EndoVascular Aneurysm Sealing (EVAS) is a relatively new technology, lacking long-term data, and concerns have been raised regarding the long-term outcome and failure modes, particularly caudal migration[8]. The longterm stability of the endograft is dependent on its ability to withstand the repetitive stresses posed by the blood flow. Previous research has demonstrated that the degree of endograft motion may differ per fixation site and between devices[9], which underlines the importance to understand the individual device dynamics to allow for adequate durability tests. To achieve long-term stability, it seems mandatory that the individual stents and chimney grafts move as a single unit. However, no study has investigated this in a clinical setting. In addition, loss of branch vessel patency may result from bending motion of the stented vessel leading to tissue injury. This study focuses on the cardiac pulsatility-induced movement and deformation of the Nellix EndoVascular Aneurysm Sealing System (Endologix, Irvine, CA, USA) in combination with chimney grafts to evaluate its proximal stability during the cardiac cycle.

\section{Methods}

Patient population This study retrospectively analyzed electrocardiogram (ECG)-gated computed tomography angiography (CTA) scans that were acquired during the postoperative period following chEVAS procedures conducted in a Dutch AAA vascular center between September 2013 and June 2016. The ECG-gated CTA scans were performed at the discretion of the treating physician. Eleven cases were available for analysis. Nine patients (mean age $77 \pm 6$ years; 8 men) underwent a primary chEVAS procedure; one of these with a Nellix-in-Nellix extension to treat failed EVAS due to caudal stent migration with a type Ia endoleak. Postoperative ECGgated CTA scans were available at a mean follow-up of $7.7 \pm 6.2$ months (range, 0-16 months). A redo chEVAS procedure (chEVAS-in-chEVAS) was conducted for two of these patients at 20 and 26 months after primary chEVAS due to respectively a type Ia endoleak with aneurysm growth and a 7-mm migration of the Nellix stents and aneurysm growth without an evident endoleak but with a reduced seal due to neck dilatation and a 2-mm downward migration of the Nellix stents[10]. Both patients were treated with a triple chimney procedure by proximal extension of the primary unilateral chEVAS device configuration. Postoperative ECG-gated CTA scans were acquired within 1 month. Data collection was approved by the local institutional review board. The patient data were previously included in the ASCEND registry[4]. 
Table 1. Baseline anatomical characteristics ${ }^{a}$ and procedural information. ${ }^{b}$

\begin{tabular}{ll}
\hline Number of patients & 9 \\
Anatomical characteristics $^{a}$ & Median (range) \\
Diameter at SMA, mm & $26(14.8-34)$ \\
Diameter at highest RA, mm & $29.7(16.3-44.9)$ \\
Diameter at lowest RA, mm & $32(19-42)$ \\
Infrarenal angulation, deg & $19(8.4-41)$ \\
Suprarenal angulation, deg & $31.7(14.5-40.4)$ \\
Maximum AAA diameter, mm & $59(51.7-106.5)$ \\
Procedural information ${ }^{b}$, & \\
primary / & \\
revision & \\
Number of chimney grafts & I: $5 \mid$ II: $3 \mid$ III: $1 /$ \\
I | II | III & I: $0 \mid$ II: $0 \mid$ III: 2 \\
Endobag fill volume, mL & $90(25-180) /$ \\
Endobag fill pressure, mmHg & $20(20-20)$ \\
Technical success & $197.5(180-250) /$ \\
& $233(216-250)$ \\
\end{tabular}

Abbreviations: RA, renal artery; AAA, abdominal aortic aneurysm.

${ }^{a}$ Aneurysm characteristics at primary chEVAS treatment.

${ }^{b}$ Procedural information including primary and revision chEVAS procedures.

Table 1 shows the baseline anatomical characteristics and procedural information. Advanta V12 covered chimney stents (Maquet Getinge Group, Hudson, NH, USA) were used in all patients. Technical success was achieved during all procedures.

Image acquisition ECG-gated CTA scans were acquired with a 256-slice CT scanner (Brilliance iCT 256 scanner, Philips Healthcare, Eindhoven, the Netherlands). Scanning parameters were similar to the conventional in-house used CTA scan protocol for EVAS follow-up and included a tube voltage of $100 \mathrm{kV}$, rotation time of 0.27 seconds, tube current time product of $63-236 \mathrm{~mA} \cdot \mathrm{s}$, collimation of $128 \times 0.625$ $\mathrm{mm}$, pitch factor of $0.16-0.18$, and reconstructed matrix size of $512 \times 512$ pixels. Slice thickness was $2.5 \mathrm{~mm}$ with $2.5 \mathrm{~mm}$ spacing between slices (increment). Retrospective gating was performed at $10 \%$ intervals from $0 \%$ to $90 \%$ of the RR-interval. For one dataset, an additional reconstruction was made with a slice thickness of $0.9 \mathrm{~mm}$ with $0.45 \mathrm{~mm}$ increment. An intravascular contrast volume of $80 \mathrm{~mL}(300 \mathrm{mg} \mathrm{I} / \mathrm{mL})$ was injected at a flow rate of $4 \mathrm{~mL} / \mathrm{s}$.

Image analysis Image analysis was performed using a previously established combination of an image registration and a segmentation algorithm[11] that was validated for motion estimation of endografts in ECG-gated CTA data[9]. The algorithm was customized to quantify motion and deformation of the Nellix stents, the chimney grafts and the stented branch vessels during the cardiac cycle. An overview of the 

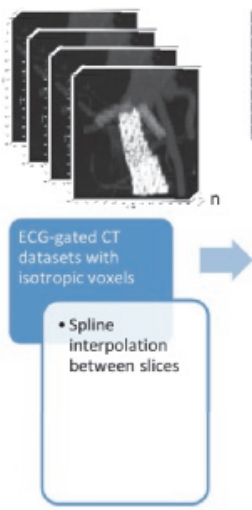
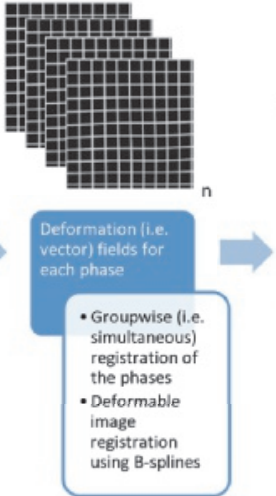
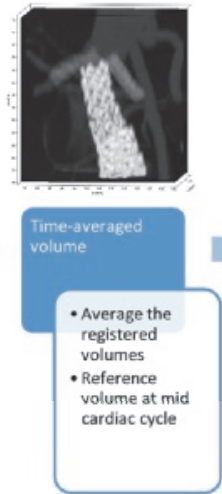
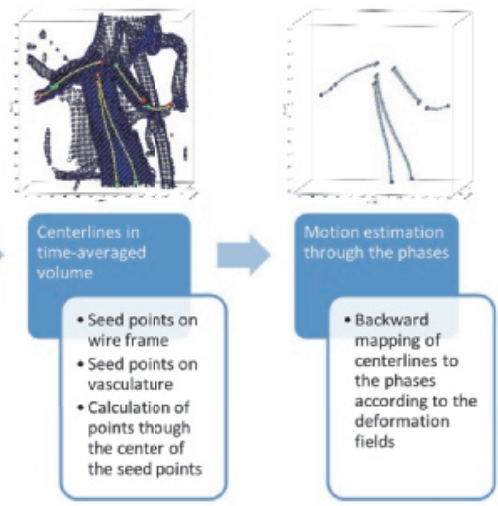

Figure 1. Flow chart showing the steps of the applied methodology for evaluation of three dimensional stent-graft motion in ECG-gated computed tomography.

applied methodology is shown in Figure 1. First, the ECG-gated datasets with a slice thickness of $2.5 \mathrm{~mm}$ were interpolated in z-direction by spline interpolation to acquire sub-mm isotropic voxels. The interpolated volumes were registered simultaneously by a deformable (i.e., elastic) registration algorithm dedicated to endograft deformation analysis[11]. Elastic (i.e., non-rigid) registration was necessary to allow for estimation of deformation, including bending of stents. The result of elastic registration is a deformation field for each reconstructed phase in the cardiac cycle, which can be used to compute the position of points in the data during the cardiac cycle. The deformation fields describe the displacement of all voxels with respect to the average of all phases, hereafter referred to as time-averaged volume, which corresponds to mid cardiac cycle.

To analyze motion and changes in geometry, lumen centerlines of the stents and branch vessels were obtained, which run through the center of the stent's wire frame and the vessel lumen boundary, respectively (Figure 1). The wire frames were segmented as a set of points in the time-averaged volume by thresholding at 600 Hounsfield units (HU). The vessel boundaries were obtained using an implementation of the marching cubes algorithm[12] in the open source functions of scikit-image[13] v.0.11 at a threshold of $250 \mathrm{HU}$. The vertices of the resulting surface mesh were used as vessel boundary points. Centerlines of the stents and branch vessels were extracted by calculating the path through the center of the respective point set by maximizing the distance to the point set. The step size of points along the centerline was set to $1 \mathrm{~mm}$. The deformation fields were applied to the centerlines by backward mapping to estimate the position of the centerlines at each phase in the cardiac cycle.

Finally, translation of the proximal segment of the Nellix stents and the proximal and distal segments of the chimney grafts; component stability expressed as distance change between the proximal ends of both Nellix stents (Nellix-to-Nellix distance) and between the proximal ends of the Nellix stents and the chimney grafts (Nellix-tochimney distance); and angulation change of the chimney grafts themselves (chimney angle), between the proximal segment of the Nellix stents and the chimney grafts 
(Nellix-to-chimney angle), and between the distal segment of the chimney grafts and the branch vessel distal to the chimney grafts (end-stent angle) were quantified during the cardiac cycle (Figure 2). Segments of 10 centerline points $(10 \mathrm{~mm})$ were used. The chimney angles were calculated in a triangular orientation as the angle between two $10-\mathrm{mm}$ vectors from a point on the centerline to two other centerline points at a fixed arm length of $10 \mathrm{~mm}$. The maximum change in angle at a certain point on the centerline (chimney deflection) was assessed as well as the maximum angle of the centerline at each phase in the cardiac cycle (peak angle change). The Nellix-tochimney and end-stent angles were calculated as the angle between the directional vectors of the centerline segments. An angle of zero indicates a straight line.

For validation purposes, an additional analysis was performed on the $0.9 \mathrm{~mm}$ slice reconstruction that was available for one of the datasets. Interpolation between the slices was not applied to this additional dataset. A comparison of cyclic motion and deformation was made between the $0.9 \mathrm{~mm}$ and $2.5 \mathrm{~mm}$ slice-reconstructions (Supplemental Resource 1).

Statistics Descriptive data were summarized as mean \pm standard deviation (range). Categorical data were expressed as numbers. Comparative measurements were performed using an independent samples $t$-test. Statistical significance was assumed when $\mathrm{p}<0.05$. The open source statistical functions of Scipy[14] v.1.1 were used with Miniconda3 as Python interpreter (Python 3).

\section{Results}

All image processing was successfully performed. Centerlines were obtained in all 11 ECG-gated CTA datasets, including 22 Nellix stents and 20 chimney grafts (left renal artery (LRA): $n=9$, right renal artery $(\mathrm{RRA}): \mathrm{n}=8$, superior mesenteric artery (SMA): $n=3)$. An example of the dynamic CTA analysis performed is shown in Figure 2. A maximum intensity projection cine-loop of the original ECG-gated CT volumes is shown in Video S1. The corresponding constructed centerline models are shown in Video S2.

Device translation and component stability during the cardiac cycle Translation during the cardiac cycle was quantified in the $\mathrm{x}-$, $\mathrm{y}$ - and $\mathrm{z}$-direction, corresponding to the right-to-left, anterior-to-posterior, and cranial-to-caudal direction, and in three dimensional (3D) space, i.e., the vector length. The mean displacement of the Nellix stents and the chimney grafts is shown in Table 2. Overall, the 3D displacement was at most $1.7 \mathrm{~mm}$ for both the Nellix stents and the chimney grafts, showing no statistically significant differences between the proximal and distal segment of the chimney grafts $(0.9 \pm 0.4 \mathrm{~mm}$ vs. $0.8 \pm 0.3 \mathrm{~mm}, \mathrm{p}=0.632)$, and between the proximal segments of the Nellix stents and the chimney grafts $(1.0 \pm 0.4 \mathrm{~mm}$ vs. $0.9 \pm 0.4 \mathrm{~mm}, \mathrm{p}=0.540)$. Distances from Nellix-to-Nellix and from Nellix-to-chimney during the cardiac cycle are shown in Figure 3. The maximum change in distance was on average $0.2 \pm 0.1 \mathrm{~mm}(0.1-0.4 \mathrm{~mm})$ from Nellix-to-Nellix and $0.2 \pm 0.1 \mathrm{~mm}(0.0$ $0.4 \mathrm{~mm})$ from Nellix-to-chimney, with no statistically significant difference $(\mathrm{p}=0.823)$. 


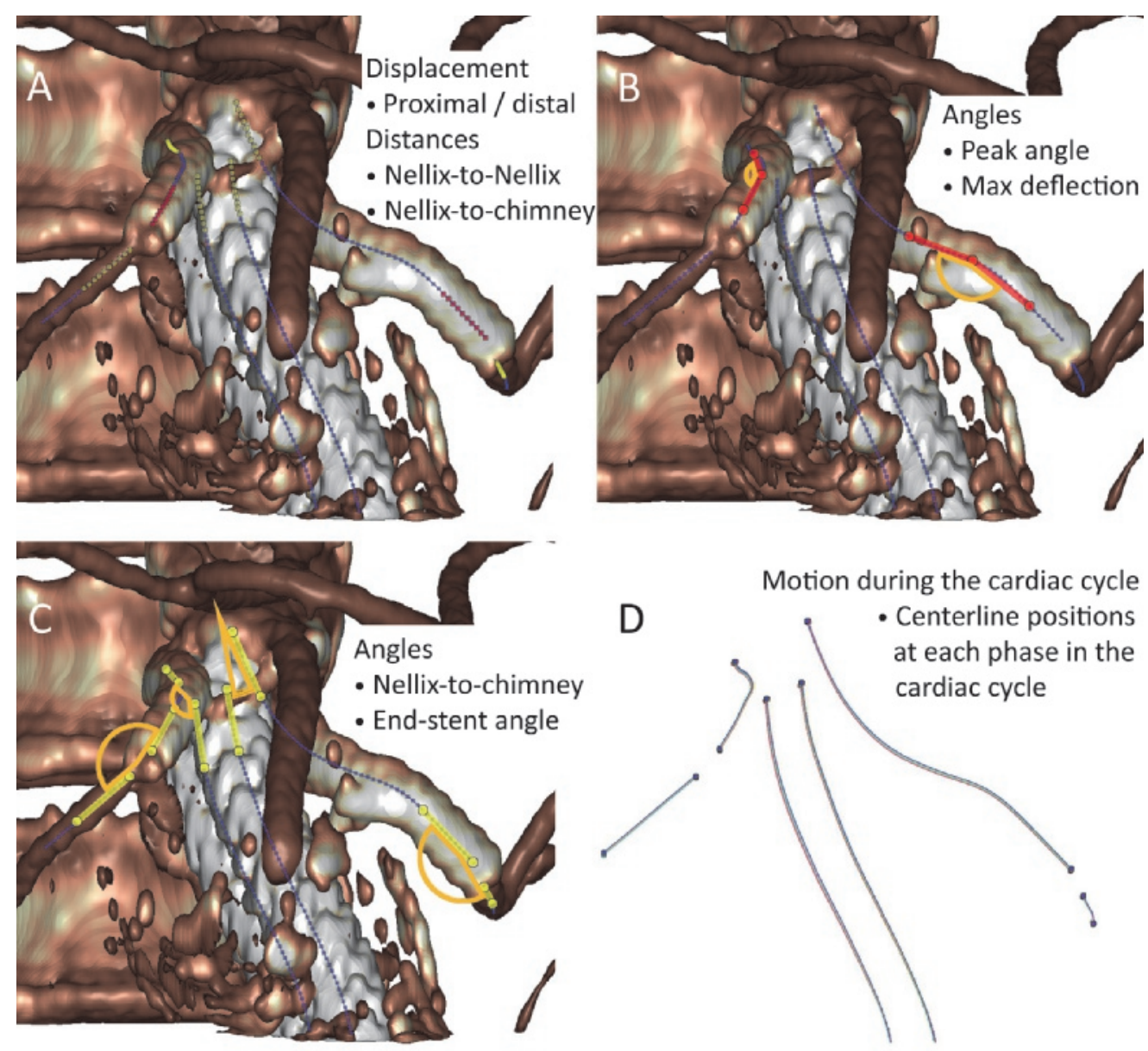

Figure 2. Dynamic CTA measurements of the Nellix stents, the chimney grafts, and the branch vessels distal to the chimney grafts. Measurements describe (A) cardiac pulsatilityinduced displacement of the Nellix stents and chimneys and distances from Nellix-to-Nellix and from Nellix-to-chimney; (B) chimney angles defined as maximum deflection angles and peak angles; and (C) angles from Nellix-to-chimney and end-stent angles from chimney to branch vessel. (D) The relative position of the centerlines during the cardiac cycle is shown for the case example (\#2) presented. Minor displacement and deformation of the Nellix stents, the chimney grafts and the branch vessel is seen during the cardiac cycle. 

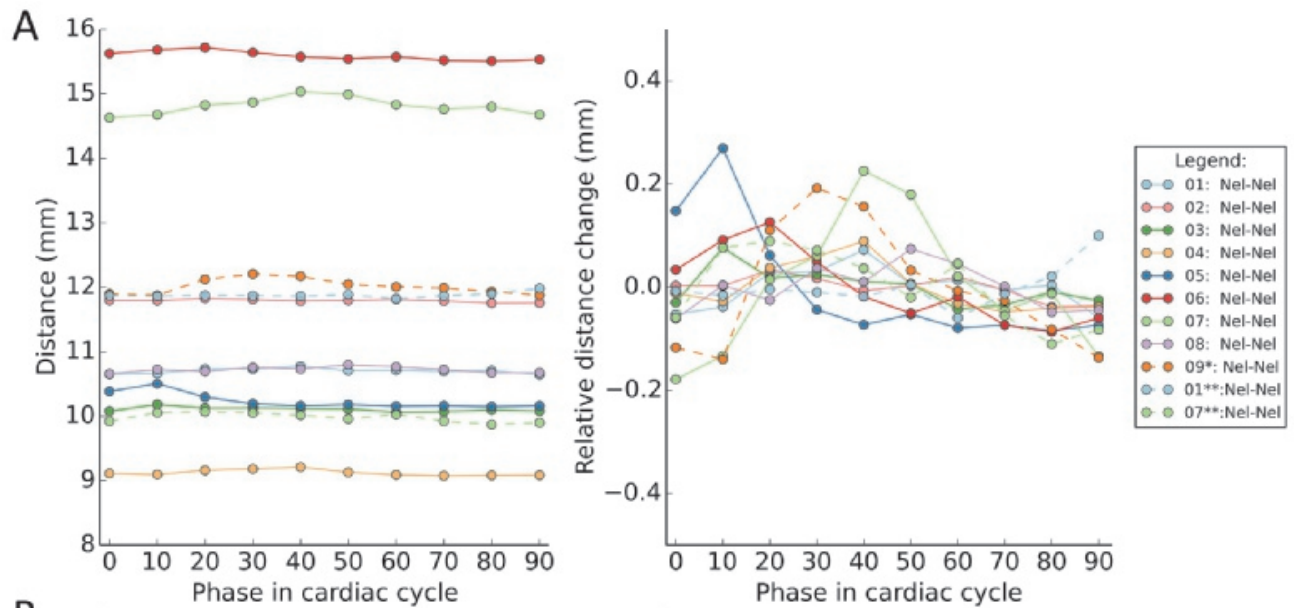

B

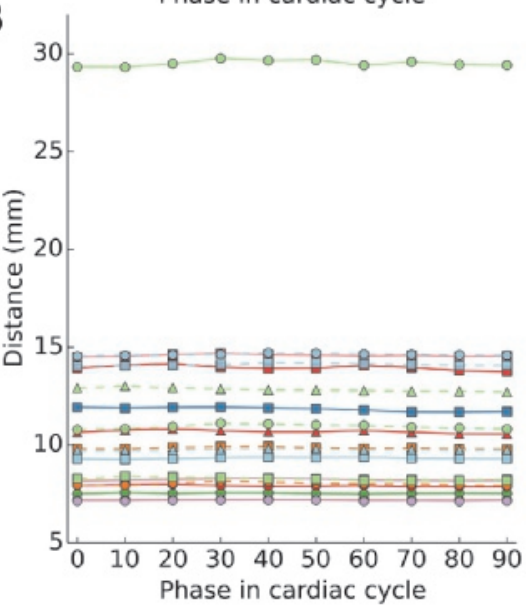

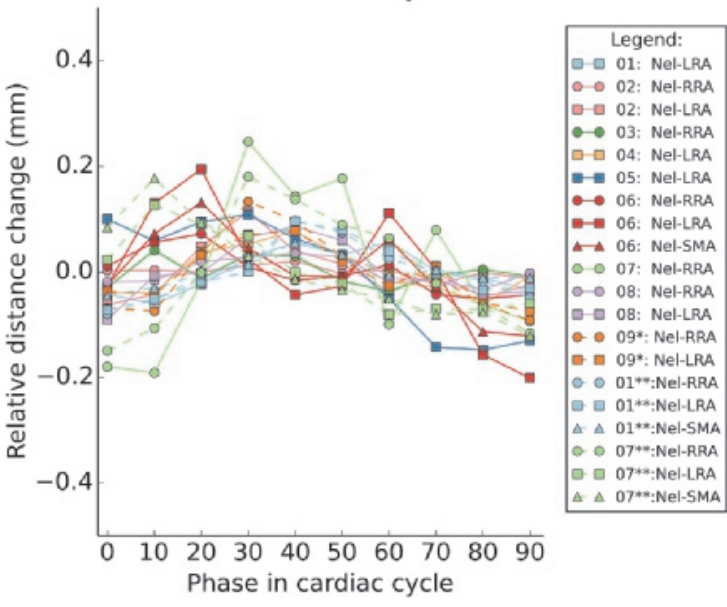

Figure 3. (A) Distance change from Nellix-to-Nellix and (B) from Nellix-to-chimney during the cardiac cycle. Absolute distances are shown (left) as well as the relative change in distance with respect to the distance at mid cardiac cycle (right). LRA, left renal artery; RRA, right renal artery; SMA, superior mesenteric artery; Nel, Nellix stent; Nel-Nel, Nellix-to-Nellix. 

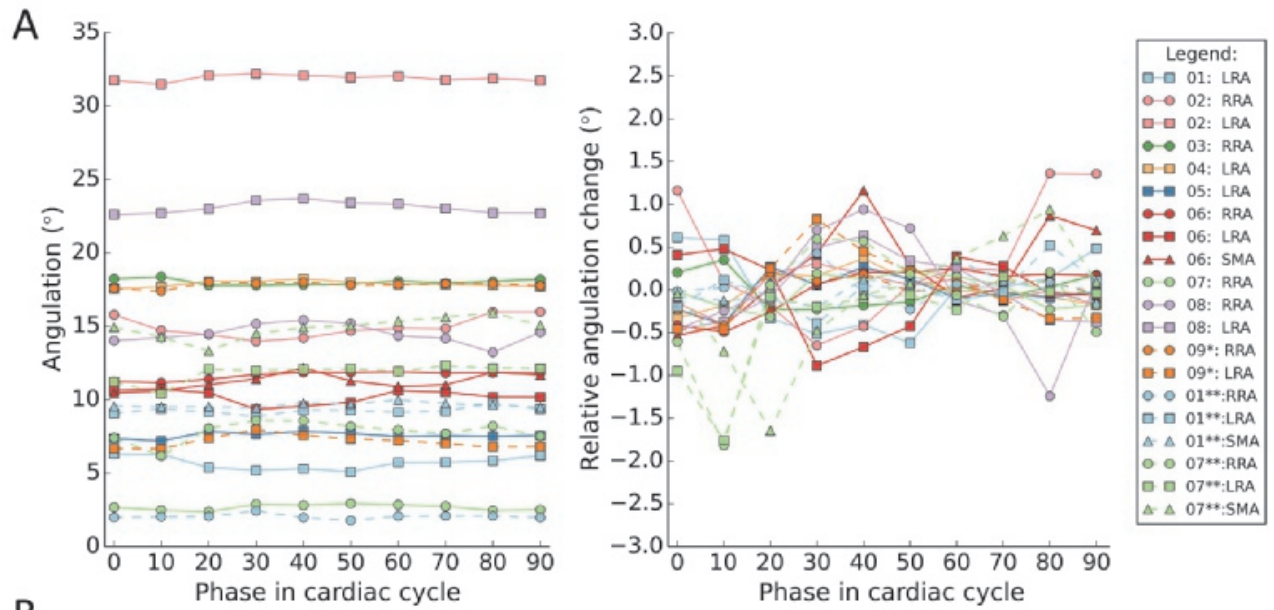

B
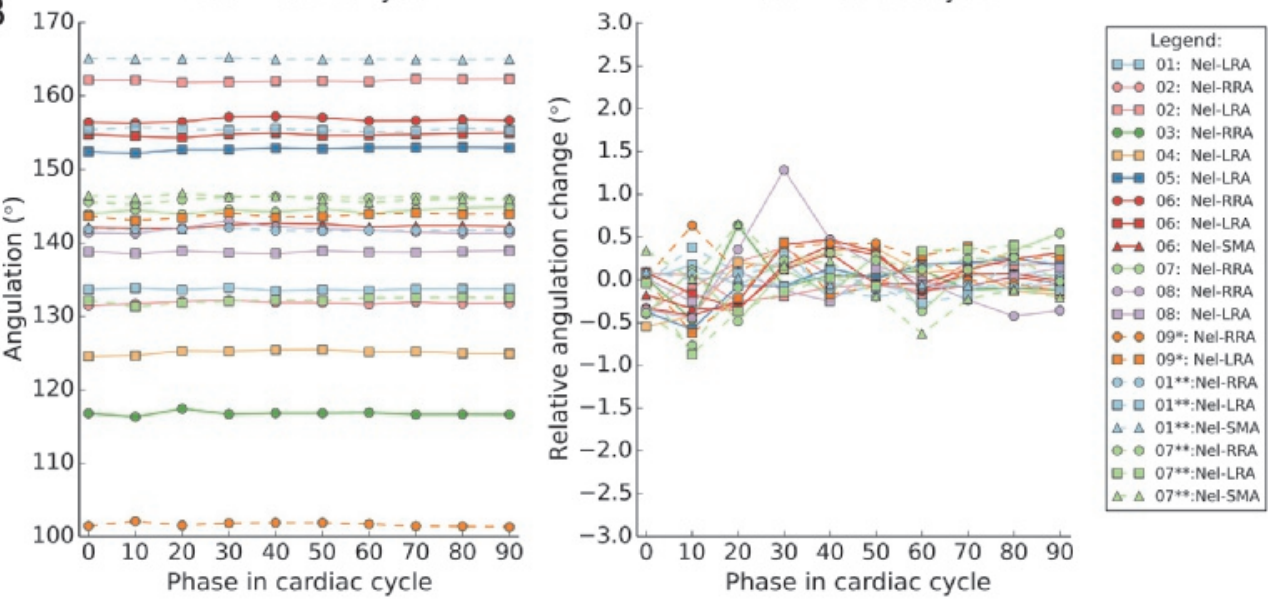

Figure 4. Bending of the chimney grafts during the cardiac cycle. Angles of (A) maximum chimney deflection and (B) Nellix-to-chimney angles are displayed. Absolute angles are shown (left) as well as the relative change in angle with respect to the angle at mid cardiac cycle (right). LRA, left renal artery; RRA, right renal artery; SMA, superior mesenteric artery; Nel, Nellix stent. 
Table 2. Translation during the cardiac cycle of the Nellix stents and the chimney grafts. ${ }^{a}$

\begin{tabular}{cllll}
\hline & \multicolumn{4}{c}{ Magnitude of displacement, mm } \\
& \multicolumn{1}{c}{ X-direction } & \multicolumn{1}{c}{ Y-direction } & Z-direction & \multicolumn{1}{c}{$3 \mathrm{D}$} \\
\hline Nellix prox, & $0.5 \pm 0.2$ & $0.5 \pm 0.2$ & $0.6 \pm 0.3$ & $0.9 \pm 0.4$ \\
left / right & $(0.2-0.9) /$ & $(0.2-1.0) /$ & $(0.2-1.2) /$ & $(0.4-1.5) /$ \\
& $0.5 \pm 0.3$ & $0.5 \pm 0.2$ & $0.6 \pm 0.3$ & $1.0 \pm 0.4$ \\
Nellix prox mean & $(0.1-0.9)$ & $(0.2-0.9)$ & $(0.1-1.2)$ & $(0.3-1.7)$ \\
& $0.5 \pm 0.2$ & $0.5 \pm 0.2$ & $0.6 \pm 0.3$ & $1.0 \pm 0.4$ \\
Chimney graft LRA, & $(0.1-0.9)$ & $(0.2-1.0)$ & $(0.1-1.2)$ & $(0.3-1.7)$ \\
prox / dist & $0.5 \pm 0.2$ & $0.5 \pm 0.2$ & $0.5 \pm 0.3$ & $0.9 \pm 0.4$ \\
& $(0.3-0.9) /$ & $(0.3-0.8) /$ & $(0.1-1.2) /$ & $(0.4-1.7) /$ \\
Chimney graft RRA, & $0.5 \pm 0.2$ & $0.5 \pm 0.2$ & $0.6 \pm 0.3$ & $0.8 \pm 0.3$ \\
prox / dist & $(0.2-0.7)$ & $(0.2-0.7)$ & $(0.1-1.2)$ & $(0.4-1.5)$ \\
& $(0.2-0.2$ & $0.6 \pm 0.2$ & $0.5 \pm 0.2$ & $0.9 \pm 0.4$ \\
Chimney graft SMA, & $0.4 \pm 0.1(0.2-0.6)$ & $(0.3-1.0) /$ & $(0.1-1.0) /$ & $(0.5-1.6) /$ \\
prox / dist & $0.5 \pm 0.3$ & $(0.1-0.8)$ & $(0.2-0.9)$ & $(0.3-1.3)$ \\
& $(0.3-0.9) /$ & $0.6 \pm 0.3$ & $0.4 \pm 0.2$ & $0.8 \pm 0.5$ \\
Chimney graft mean, & $0.5 \pm 0.3$ & $(0.4-1.0) /$ & $(0.1-0.6) /$ & $(0.4-1.5) /$ \\
prox / dist & $(0.2-0.8)$ & $0.6 \pm 0.3$ & $0.4 \pm 0.3$ & $0.8 \pm 0.5$ \\
& $(0.5 \pm 0.2$ & $(0.1-0.8)$ & $(0.2-0.8)$ & $(0.3-1.4)$ \\
& $0.5 \pm 0.2$ & $0.6 \pm 0.2$ & $0.5 \pm 0.3$ & $0.9 \pm 0.4$ \\
& $(0.2-0.8)$ & $(0.3-1.0) /$ & $(0.1-1.2) /$ & $(0.4-1.7) /$ \\
& $0.5 \pm 0.2$ & $0.5 \pm 0.3$ & $0.8 \pm 0.3$ \\
& $(0.1-0.8)$ & $(0.1-1.2)$ & $(0.3-1.5)$ \\
\hline
\end{tabular}

Abbreviations: LRA, left renal artery; RRA, right renal artery; SMA, superior mesenteric artery; prox, proximal; dist, distal; 3D, three dimensional.

${ }^{a}$ Data are presented as the means \pm standard deviation (range).

The maximum change in distance was greatest in the post-chEVAS scan of a patient (\#7) in which stent migration with a type Ia endoleak was identified (Nellix-to-Nellix: $0.4 \mathrm{~mm}$; Nellix-to-chimney $0.4 \mathrm{~mm}$ ).

Chimney angulation during the cardiac cycle Bending of the chimney grafts themselves and the Nellix-to-chimney angulation during the cardiac cycle was quantified (Figure 4). The chimney deflection angles of the LRA, RRA and SMA varied on average by $1.1 \pm 0.4^{\circ}\left(0.7-1.9^{\circ}\right), 1.2 \pm 0.8^{\circ}\left(0.6-2.4^{\circ}\right)$ and $1.6 \pm 0.8^{\circ}\left(0.5-2.6^{\circ}\right)$, respectively. The deflection angles of the renal chimney grafts did not significantly differ from the SMA chimney grafts $\left(1.2 \pm 0.6^{\circ}\right.$ vs. $\left.1.6 \pm 0.8^{\circ}, \mathrm{p}=0.296\right)$. Overall, the mean change in chimney deflection angle was $1.2 \pm 0.7^{\circ}\left(0.5-2.6^{\circ}\right)$. The average length of the chimney centerlines was $37.5 \pm 14.3 \mathrm{~mm}(22-62 \mathrm{~mm})$. Deflection angles were located on average at $48 \pm 15 \%(23-80 \%)$ of the chimney graft relative to the proximal end of the chimney.

The minimum and maximum peak angles of the LRA, RRA and the SMA chimney grafts were on average $21.0 \pm 11.2^{\circ}\left(7.2-36.6^{\circ}\right), 16.8 \pm 5.8^{\circ}\left(2.4-23.1^{\circ}\right)$, and $14.9 \pm$ $3.8^{\circ}\left(10.1-19.5^{\circ}\right)$ vs. $21.7 \pm 11.2^{\circ}\left(7.9-37.4^{\circ}\right), 17.7 \pm 5.8^{\circ}\left(3.4-24.5^{\circ}\right)$, and $15.7 \pm$ $4.0^{\circ}\left(10.6-20.5^{\circ}\right)$, respectively, with no significant differences between renal and SMA 

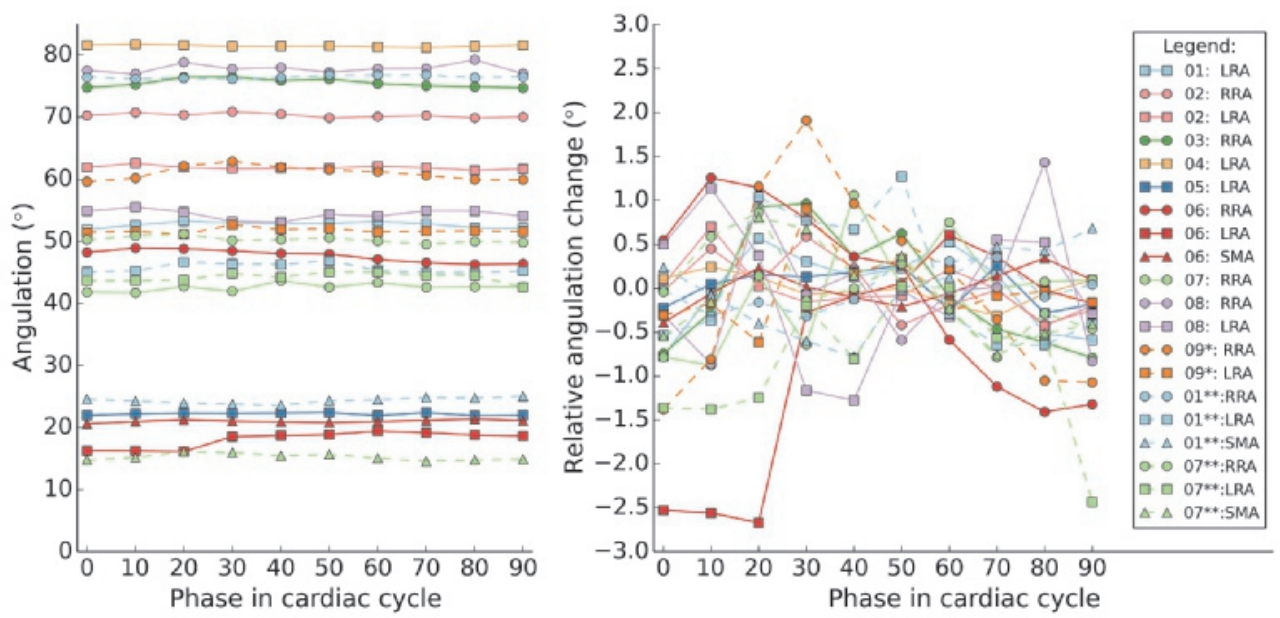

Figure 5. End-stent angle relative to the distal native branch vessel. Absolute angles are shown (left) as well as the relative change in angle with respect to the angle at mid cardiac cycle (right). LRA, left renal artery; RRA, right renal artery; SMA, superior mesenteric artery.

chimney grafts for both minimum $(\mathrm{p}=0.483)$ and maximum $(\mathrm{p}=0.481)$ peak angles. The absolute change in peak angle of the LRA, RRA and the SMA chimney grafts was on average $0.8 \pm 0.3^{\circ}\left(0.3-1.5^{\circ}\right), 0.9 \pm 0.4^{\circ}\left(0.5-1.6^{\circ}\right)$, and $0.8 \pm 0.2^{\circ}\left(0.5-1.0^{\circ}\right)$, respectively. At mid cardiac cycle, the LRA, RRA, and SMA peak angles were on average located at $57 \pm 10 \%$ (44-68\%), $55 \pm 11 \%$ (42-76\%), and $44 \pm 1 \%(43-46 \%)$ of the chimney graft relative to the proximal end of the chimney. The location of the peak angle during the cardiac cycle changed by $0.4 \pm 0.3 \mathrm{~mm}(0.1-1.2 \mathrm{~mm}), 0.6 \pm$ $0.5 \mathrm{~mm}(0.1-1.8 \mathrm{~mm})$ and $1.3 \pm 1.1 \mathrm{~mm}(0.1-2.7 \mathrm{~mm})$ for the LRA, RRA, and SMA, respectively.

The Nellix-to-chimney angles for the LRA, RRA and SMA varied on average by respectively $0.7 \pm 0.3^{\circ}\left(0.4-1.3^{\circ}\right), 1.0 \pm 0.3^{\circ}\left(0.5-1.7^{\circ}\right)$, and $0.8 \pm 0.4^{\circ}\left(0.3-1.3^{\circ}\right)$ during the cardiac cycle. There was no significant difference between renal and SMA chimney grafts $\left(0.9 \pm 0.3^{\circ}\right.$ vs. $\left.0.8 \pm 0.4^{\circ}, \mathrm{p}=0.767\right)$. Minimum and maximum Nellixto-chimney angles for the LRA, RRA and the SMA chimney grafts were on average $143.8 \pm 12.0^{\circ}\left(124.5-161.8^{\circ}\right), 134.6 \pm 16.6^{\circ}\left(101.3-156.3^{\circ}\right)$, and $150.8 \pm 10.1^{\circ}(141.9$ $\left.164.9^{\circ}\right)$ vs. $144.5 \pm 11.9^{\circ}\left(125.4-162.3^{\circ}\right), 135.6 \pm 16.7^{\circ}\left(102.1-157.2^{\circ}\right)$ and $151.5 \pm$ $9.8^{\circ}\left(142.7-165.2^{\circ}\right)$, respectively. There were no significant differences between renal and SMA chimney grafts for both minimum $(\mathrm{p}=0.253)$ and maximum $(\mathrm{p}=0.253)$ Nellix-to-chimney angles.

End-stent angle during the cardiac cycle The end-stent angles relative to the distal native branch vessels were quantified (Figure 5). The end-stent angles for the LRA, RRA, and SMA varied on average by respectively $1.7 \pm 0.9^{\circ}\left(0.5-3.3^{\circ}\right), 1.9$ $\pm 0.8^{\circ}\left(0.7-3.3^{\circ}\right)$, and $1.3 \pm 0.4^{\circ}\left(0.7-1.6^{\circ}\right)$ during the cardiac cycle. The difference 
was not statistically significant between the RAs and the SMAs $\left(1.8 \pm 0.8^{\circ}\right.$ vs. 1.3 $\left.\pm 0.4^{\circ}, \mathrm{p}=0.316\right)$. Overall, the end-stent angles varied on average by $1.7 \pm 0.8^{\circ}$ $\left(0.5-3.3^{\circ}\right)$. The end-stent angles exhibited significantly greater change during the cardiac cycle compared to the Nellix-to-chimney angles $\left(1.7 \pm 0.8^{\circ}\right.$ vs. $0.8 \pm 0.3^{\circ}$, $\mathrm{p}<0.001)$. Minimum and maximum end-stent angles for the LRA, RRA and the SMA were on average $47.2 \pm 18.4^{\circ}\left(16.1-81.2^{\circ}\right), 61.9 \pm 13.5^{\circ}\left(41.7-77.0^{\circ}\right)$, and $19.6 \pm$ $3.8^{\circ}\left(14.6-23.6^{\circ}\right)$ vs. $48.8 \pm 18.0^{\circ}\left(19.4-81.7^{\circ}\right), 63.8 \pm 13.3^{\circ}\left(43.7-79.3^{\circ}\right), 20.9 \pm 3.7^{\circ}$ (16.1-25. $\left.1^{\circ}\right)$. The renal end-stent angles were greater compared to the SMA end-stent angles for both the minimum $\left(54.1 \pm 17.9^{\circ}\right.$ vs. $\left.19.6 \pm 3.8^{\circ}, \mathrm{p}=0.006\right)$ and maximum $\left(55.9 \pm 17.6^{\circ}\right.$ vs. $\left.20.9 \pm 3.7^{\circ}, \mathrm{p}=0.004\right)$ end-stent angles.

Comparison of a $\mathbf{2 . 5}$ and $\mathbf{0 . 9} \mathrm{mm}$-slice reconstruction All measurements were repeated on the $0.9 \mathrm{~mm}$-slice reconstruction to compare with the analysis of the 2.5 $\mathrm{mm}$ slice-reconstruction. The highest deviations were $0.11 \mathrm{~mm}$ for the magnitude of displacement, $0.06 \mathrm{~mm}$ for distance change, $0.10^{\circ}$ for chimney deflection angle change, $0.25^{\circ}$ for the change in peak angle, $0.23^{\circ}$ for Nellix-to-chimney angle change, and $0.48^{\circ}$ for the end-stent angle change. A comprehensive description of the comparison can be found in Supplemental Resource 1.

\section{Discussion}

The present study evaluated the cardiac pulsatility-induced displacement and deformation of the proximal sealing and fixation zone of the Nellix EndoVascular Aneurysm Sealing System combined with chimney grafts. To our knowledge, this is the first study to perform such analysis in multiphasic ECG-gated CTA data of patients treated with chEVAS. The proximal part of the Nellix stents and chimney grafts exhibited minimal displacement during the cardiac cycle (magnitude of displacement on average $\leq 1.0 \mathrm{~mm}$ ), with the device components moving together as a single unit.

It is important to emphasize that these results, although showing a stable situation, do not predict the potential for future migration, as other forces may contribute to this phenomenon. Lateral bending is considered as one of the driving factors behind caudal migration in EVAS, leading to type Ia endoleak[15]. Stiffness of the stents and polymer-filled endobags seems to be a key factor. This is reflected by the introduction of the thrombus index in the instructions for use. Moreover, it was recently advised to increase the rigidity of the system in case of Nellix-in-Nellix revision for distal migration, in order to minimize the risk of ongoing lateral bending of the stents[16,17]. Little is known about the risk of migration after chEVAS[4]. In the present study sample, two patients required a chEVAS revision, one of which presenting evident migration of the Nellix stents. Both of them were initially treated with a short seal zone. Two years after revision both patients showed no signs of failure[10]. This supports the analysis of the post-revision dynamic CTA scan performed in this study showing no deviant cyclic behavior from other chEVAS cases. Longitudinal studies using ECG-gated CTA scanning after chEVAS are indicated to further assess the predictive value of this scanning protocol. A progressive motion during the cardiac cycle might be an early indicator for decreased positional stability and as such the used 
scan technique could be of great value during follow-up of these and other complex procedures.

The Nellix-to-chimney angles were virtually constant during the cardiac cycle $(0.9$ $\left.\pm 0.4^{\circ}\right)$ and the chimney grafts presented little bending deformation $\left(1.2 \pm 0.7^{\circ}\right)$, which was supported by observing comparable translation at the proximal and distal ends of the chimney grafts. This may be favorable to prevent distortion or stent fractures by bending fatigue on the long-term. From our present work, it seems that double and triple chimney configurations exhibit slightly greater chimney deflection angles compared to single chimney configurations (Figure 4), which may be related to the greater length of the chimney grafts that were used in the double and triple configurations compared to the single configurations $(41.0 \pm 14.2 \mathrm{~mm}$ vs. $27.0 \pm 7.5$ $\mathrm{mm}, \mathrm{p}=0.065)$; this may be important to consider for chimney standardization and device durability testing. These observations manifest chEVAS as a stable configuration during the cardiac cycle. However, it should be noted that the Advanta V12 graft is a relatively stiff stent compared to a self-expanding stent or a more flexible balloon expandable stent (e.g., VBX, Bentley). Further research is necessary to investigate potential differences with other chimney graft configurations.

Our observations further show that the motion of the native side vessels is damped after chEVAS as preoperative renal artery movement has been reported to be in the order of 1 to $3 \mathrm{~mm}[18,19]$. Similarly, fenestrated endografts have been shown to limit side branch motion, which may be important with regard to branch vessel patency[18]. It was previously shown by Itoga et al.[20] that after EVAS the Nellix stents undergo minimal pulsatile motion between mid-diastole and peak-systole (magnitude of movement $<0.5 \mathrm{~mm})$. In the present study, albeit still small, we observed fairly greater magnitudes of motion (up to $1.7 \mathrm{~mm}$ ). This might be explained by the transrenal positioning of the stents and thus the greater proximity of the stents to the heart compared to the infrarenal fixated stents in the study by Itoga et al[20]. Interestingly, because the Nellix stents were fixated infrarenally, this study further found a mechanical damping effect of the Nellix device on renal artery movement. This in contrast to conventional transrenal or infrarenal endografts that do not seem to alter renal artery motion[18]. The renal branch angle change due to pulsatile motion reported in the case series by Itoga et al.[20] was similar to the Nellix-to-chimney angle change reported in the present study, indicating that the mechanical damping of renal artery movement is similar with EVAS and chEVAS, whereas a damping of SMA movement was observed with chEVAS only. In chEVAS, this damping effect could contribute to a more stable configuration and therewith lead to less stent migration.

Notably, compared to the Nellix-to-chimney angles, the end-stent angles relative to the distal native branch vessels exhibited considerable variation (up to $3.3^{\circ}$ ). Although the inherent stiffness of the chimney stents limited cyclic chimney angulation, it may have induced an inflection point at the transition from chimney to vessel, as reflected by the more apparent end-stent angle. This may raise concerns regarding tissue irritation on the long term that might lead to stenosis or occlusion[19,21]. Reintervention due to renal occlusion or stenosis appears to be relatively common after chimney procedures[4,22]. The ASCEND registry[4] showed compression of the renal chimney grafts as the most prevalent cause of reintervention, although this concerned only 6 out a total of 295 patients. However, the follow-up period of the ASCEND 
registry is limited.

This study is limited in evaluating distinct postoperative scans only due to the lack of preoperative dynamic imaging and longitudinal dynamic follow-up scans. However, the present study was not designed to evaluate the effect of endograft implantation on native vessel motion or to assess changes over time but instead to evaluate the proximal stability of the chEVAS configuration during the cardiac cycle. This study contributed to the understanding of in vivo pulsatile movement of the Nellix device, the chimney grafts and the branch vessels, which is essential in improving preclinical testing and future device design. Furthermore, we were limited by the data reconstructions that were available at $2.5 \mathrm{~mm}$-slice reconstructions only, except for one dataset that by its raw data could also be reconstructed at $0.9 \mathrm{~mm}$ slices. Although we interpolated between slices, the original slice thickness may have affected the accuracy of our measurements in z-direction. However, the cardiac pulsatility-induced motions were found to be similar in $\mathrm{x}$ - and $\mathrm{y}$-direction compared to the $\mathrm{z}$-direction and comparison of the 0.9 and $2.5 \mathrm{~mm}$-slice reconstruction found minimal differences between the measurements.

The methodology applied in our present work utilizes an automated 3D approach to assess motion throughout the reconstructed volumes, which transcends previous 2D approaches. Also, motion and deformation were assessed during the whole cardiac cycle rather than during two phases of the cardiac cycle only. Future studies employing this methodology to investigate changes over time are awaited to evaluate device efficacy and durability of different endovascular procedures (e.g., chimney, fenestrated, branched configurations).

\section{Conclusion}

Only minimal cyclical changes in distance and angulation between the proximal chEVAS device components occur during the cardiac cycle. These observations manifest chEVAS as a stable configuration during the cardiac cycle. The chimney grafts themselves exhibited little cyclic bending, which may be favorable for preventing longterm metal fatigue. Still, the end-stent angles relative to the distal native branch vessels exhibited considerable variation, which may raise concerns regarding branch occlusion or stenosis. Longitudinal studies using ECG-gated CTA scanning after chEVAS are indicated to further assess the predictive value of this scanning protocol for positional stability and mechanical durability.

\section{Acknowledgments}

The authors thank Marije Voskamp (Research assistant, Department of Vascular Surgery, Rijnstate), Daphne van der Veen (Research nurse, Department of Vascular Surgery, Rijnstate), and F. Wortel (Laboratory technician, Department of Radiology, Rijnstate) for their assistance. 


\section{Declaration of conflicting interests}

M.M.P.J. Reijnen is consultant for Endologix, Bentley Innomed and Terumo Aortic. R.H. Geelkerken is consultant for Terumo Aortic.

\section{Funding}

This study was supported in part by an unrestricted grant from Endologix Inc. and in part by the PPP Allowance made available by Health Holland, Top Sector Life Sciences \& Health, to stimulate public-private partnerships.

\section{References}

1. De Bruin JL, Brownrigg JR, Patterson BO, Karthikesalingam A, Holt PJ, Hinchliffe RJ, et al. The Endovascular Sealing Device in Combination with Parallel Grafts for Treatment of Juxta/Suprarenal Abdominal Aortic Aneurysms: Short-term Results of a Novel Alternative. Eur J Vasc Endovasc Surg. 2016;52.

2. Roy IN, Gharib M, Zerwes S, Jakob R, Torella F, McWilliams RG, et al. Anatomical Applicability of Endovascular Aneurysm Sealing Techniques in a Consecutive Cohort of Fenestrated Endovascular Aneurysm Repairs. J Endovasc Ther. 2017;24:773-8.

3. Lindblad B, Bin Jabr A, Holst J, Malina M. Chimney Grafts in Aortic Stent Grafting: Hazardous or Useful Technique? Systematic Review of Current Data. Eur J Vasc Endovasc Surg. 2015;50:722-31.

4. Thompson M, Youssef M, Jacob R, Zerwes S, Reijnen M, Szopinski P, et al. Early Experience With Endovascular Aneurysm Sealing in Combination With Parallel Grafts for the Treatment of Complex Abdominal Aneurysms: The ASCEND Registry. J Endovasc Ther. 2017;24:764-772.

5. Boersen JT, Donselaar EJ, Groot Jebbink E, Starreveld R, Overeem SP, Slump CH, et al. Benchtop quantification of gutter formation and compression of chimney stent grafts in relation to renal flow in chimney endovascular aneurysm repair and endovascular aneurysm sealing configurations. J Vasc Surg. 2017;66:1565-1573.e1.

6. Paraskevas KI, Karouki M, Rehman A, Torella F, McWilliams RG, England A, et al. Endovascular Aneurysm Sealing (EVAS) Alone or in Combination with Chimney Grafts (chEVAS) for Treating Complications of Previous Endovascular Aneurysm Repair (EVAR) Procedures. Cardiovasc Intervent Radiol. 2018;41:1015-20.

7. Joseph SZ, Kwok CHR, Hockley JA, Garbowski MW, Ferguson J, Samuelson S, et al. A Technique of "Chimney Nellix" for the Management of Type 1a Endoleak After EVAR. J Vasc Interv Radiol. 2018;29:623-7.

8. Verhoeven ELGG, Mani K. New Technology Failures: Who to Blame or Time to be Cautious? Eur J Vasc Endovasc Surg. 2018;56:318-9.

9. Koenrades MA, Struijs EM, Klein A, Kuipers H, Reijnen MMPJ, Slump CH, et al. Quantitative Stent Graft Motion in ECG Gated CT by Image Registration and Segmentation: In Vitro Validation and Preliminary Clinical Results. Eur J Vasc Endovasc Surg. 2019; [Epub ahead of print].

10. van de Velde L, Zoethout AC, Lardenoije J-WHP, Reijnen MMPJ. Secondary Endovascular Aneurysm (EVAS) Sealing in Combination With Chimney Grafts to Treat Failed Chimney EVAS. J Endovasc Ther. 2019;26:265-8.

11. Klein A, Renema W, Vliet JA, Oostveen LJ, Hoogeveen Y, Schultze Kool LJ, et al. Motion Calculations on Stent Grafts in AAA. In: Grundmann RT, editor. Diagnosis, Screening and Treatment of Abdominal, Thoracoabdominal and Thoracic Aortic Aneurysms. [Rijeka]: InTechOpen; 2011. p. $125-44$.

12. Lorensen WE, Cline HE. Marching Cubes: A High Resolution 3D Surface Construction Algorithm. SIGGRAPH Comput Graph. 1987;21:163-9. 
13. van der Walt S, Schönberger JL, Nunez-Iglesias J, Boulogne F, Warner JD, Yager N, et al. scikit-image: Image processing in Python. PeerJ. 2014;2:e453.

14. Jones E, Oliphant T, Peterson P. SciPy: Open source scientific tools for Python. 2001. Available from: http://www.scipy.org/.

15. van den Ham LH, Holden A, Savlovskis J, Witterbottom A, Ouriel K, Reijnen MMPJ, et al. Editor's Choice - Occurrence and Classification of Proximal Type I Endoleaks After EndoVascular Aneurysm Sealing Using the Nellix ${ }^{\mathrm{TM}}$ Device. Eur J Vasc Endovasc Surg. 2017;54:729-36.

16. Donselaar EJ, Holden A, Zoethout AC, Zeebregts CJ, Reijnen MMPJ. Feasibility and Technical Aspects of Proximal Nellix-in-Nellix Extension for Late Caudal Endograft Migration. J Endovasc Ther. 2016;24:210-7.

17. Zoethout AC, Zerwes S, Zeebregts CJAM, Heyligers JMM, De Vries JPJM, Oberhuber A, et al. Preliminary outcome of Nellix-in-Nellix extensions in patients treated with failed endovascular aneurysm sealing. J Vasc Surg. 2019; [Epub ahead of print].

18. Muhs BE, Vincken KL, Teutelink A, Verhoeven ELG, Prokop M, Moll FL, et al. Dynamic Cine-Computed Tomography Angiography Imaging of Standard and Fenestrated Endografts: Differing Effects on Renal Artery Motion. Vasc Endovascular Surg. 2008;42:25-31.

19. Muhs BE, Teutelink A, Prokop M, Vincken KL, Moll FL, Verhagen HJM. Endovascular Aneurysm Repair Alters Renal Artery Movement: A Preliminary Evaluation Using Dynamic CTA. J Endovasc Ther. 2006;13:476-80.

20. Itoga NK, Suh G-Y, Cheng CP. Stabilization of the Abdominal Aorta During the Cardiac Cycle with the Sac-Anchoring Nellix Device. Ann Vasc Surg. 2018;52:312.e7-312.e12.

21. Ullery BW, Suh G-Y, Lee JT, Liu B, Stineman R, Dalman RL, et al. Geometry and respiratory-induced deformation of abdominal branch vessels and stents after complex endovascular aneurysm repair. J Vasc Surg. 2015;61:875-85.

22. Donas KP, Torsello GB, Piccoli G, Pitoulias GA, Torsello GF, Bisdas T, et al. The PROTAGORAS study to evaluate the performance of the Endurant stent graft for patients with pararenal pathologic processes treated by the chimney/snorkel endovascular technique. J Vasc Surg. $2016 ; 63: 1-7$. 


\section{Appendix A}

The following are the Supplementary data to this chapter:

Supplemental Resource 1: Comparison of a 2.5 and $0.9 \mathrm{~mm}$-slice reconstruction. Supplemental Video S1 and S2.

Supplemental videos can be accessed online:

Video S1: https://vimeo.com/363934418

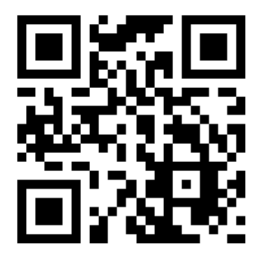

Video S2: https://vimeo.com/363935804

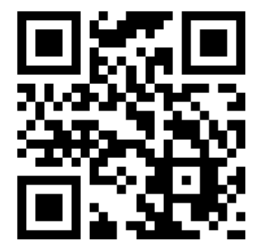




\section{Supplemental Resource 1: Comparison of a 2.5 and $0.9 \mathrm{~mm}$-slice reconstruction}

This document provides a comparison of the dynamic CTA measurements obtained from a 2.5 and $0.9 \mathrm{~mm}$-slice reconstruction of the dataset that was acquired for patient \#1 after chEVAS revision with a triple chimney procedure.

Qualitative comparison of CT data Figure S1 displays a qualitative comparison of a single volume obtained from the $2.5 \mathrm{~mm}$-slice $(2.5 \mathrm{~mm}$ increment) reconstruction before and after interpolation in z-direction and a volume obtained from the $0.9 \mathrm{~mm}$ slice $(0.45 \mathrm{~mm}$ increment) reconstruction without interpolation.
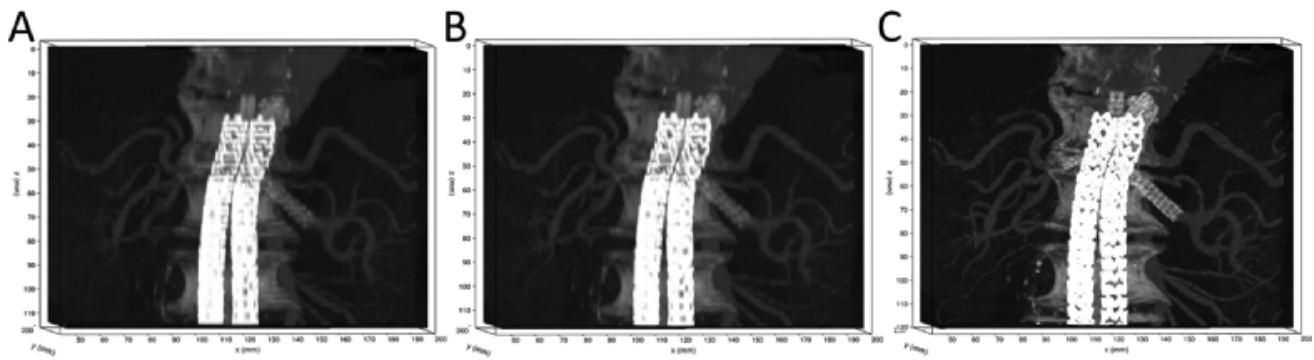

Figure S1. Maximum intensity projections of a single volume at $20 \%$ of the cardiac cycle displaying the volume of the $2.5 \mathrm{~mm}$-slice reconstruction without (A) and with (B) interpolation and the volume of the $0.9 \mathrm{~mm}$-slice reconstruction without interpolation (C).

Quantitative comparison of measurements A quantitative comparison was performed between measurements as obtained from the interpolated $2.5 \mathrm{~mm}$-slice reconstruction and the $0.9 \mathrm{~mm}$-slice reconstruction.

Displacement Figure S2 compares the $\mathrm{x}-\mathrm{y}-\mathrm{z}$ displacement, corresponding to the right $(-)$ to left $(+)$, anterior $(-)$ to posterior $(+)$, and cranial $(-)$ to caudal $(+)$ direction, of the analyzed proximal and distal parts of the Nellix stents and chimney grafts. The magnitude of displacement (3D) of the $2.5 \mathrm{~mm}$-slice reconstruction deviated on average from the $0.9 \mathrm{~mm}$-slice reconstruction by $0.05 \pm 0.03 \mathrm{~mm}$ and $0.05 \pm 0.04 \mathrm{~mm}$ for the Nellix stents and the chimney grafts, respectively.

Distance change The cyclic distance change from Nellix-to-Nellix and from Nellix-to-chimney was compared and is displayed in Figure S3. The distance change deviated by $0.06 \mathrm{~mm}$ from Nellix-to-Nellix and on average by $0.03 \pm 0.0 \mathrm{~mm}$ from Nellix-to-chimney.

Chimney deflection angle, chimney peak angle, and Nellix-to-chimney angle Chimney deflection angles and Nellix-to-chimney angles of the two reconstructions are shown in Figure S4. The deflection angle change deviated on average 
by $0.05 \pm 0.04^{\circ}$. Peak angle change of the chimney grafts deviated on average by $0.13 \pm 0.09^{\circ}$. The Nellix-to-chimney angle change deviated by $0.16 \pm 0.04^{\circ}$.

End-stent angle Figure S5 compares the end-stent angles relative to the distal native branch vessels. The change in end-stent angle deviated on average by $0.28 \pm 0.17^{\circ}$.

\section{Discussion}

Minimal differences were found between the measurements performed on the $0.9 \mathrm{~mm}$ slice and the interpolated $2.5 \mathrm{~mm}$-slice reconstruction. The only notable difference was found in the absolute values of the LRA and the RRA maximum chimney deflection angles, which could be explained by a different location of the detected maximum deflection angle (LRA: at $25 \%$ vs. $33 \%$, RRA: at $29 \%$ vs. $70 \%$ from the proximal end of the chimney graft). Most likely, small differences in the course and the displacement of the centerline have resulted in different locations of maximum chimney graft deflection. Nevertheless, the change in deflection angle during the cardiac cycle was similar, resulting in no discrepancy in the results. Even though we were only able to compare measurements for one dataset, the present comparison provides confidence that in this study the $2.5 \mathrm{~mm}$-slice reconstructions provide reliable results. 

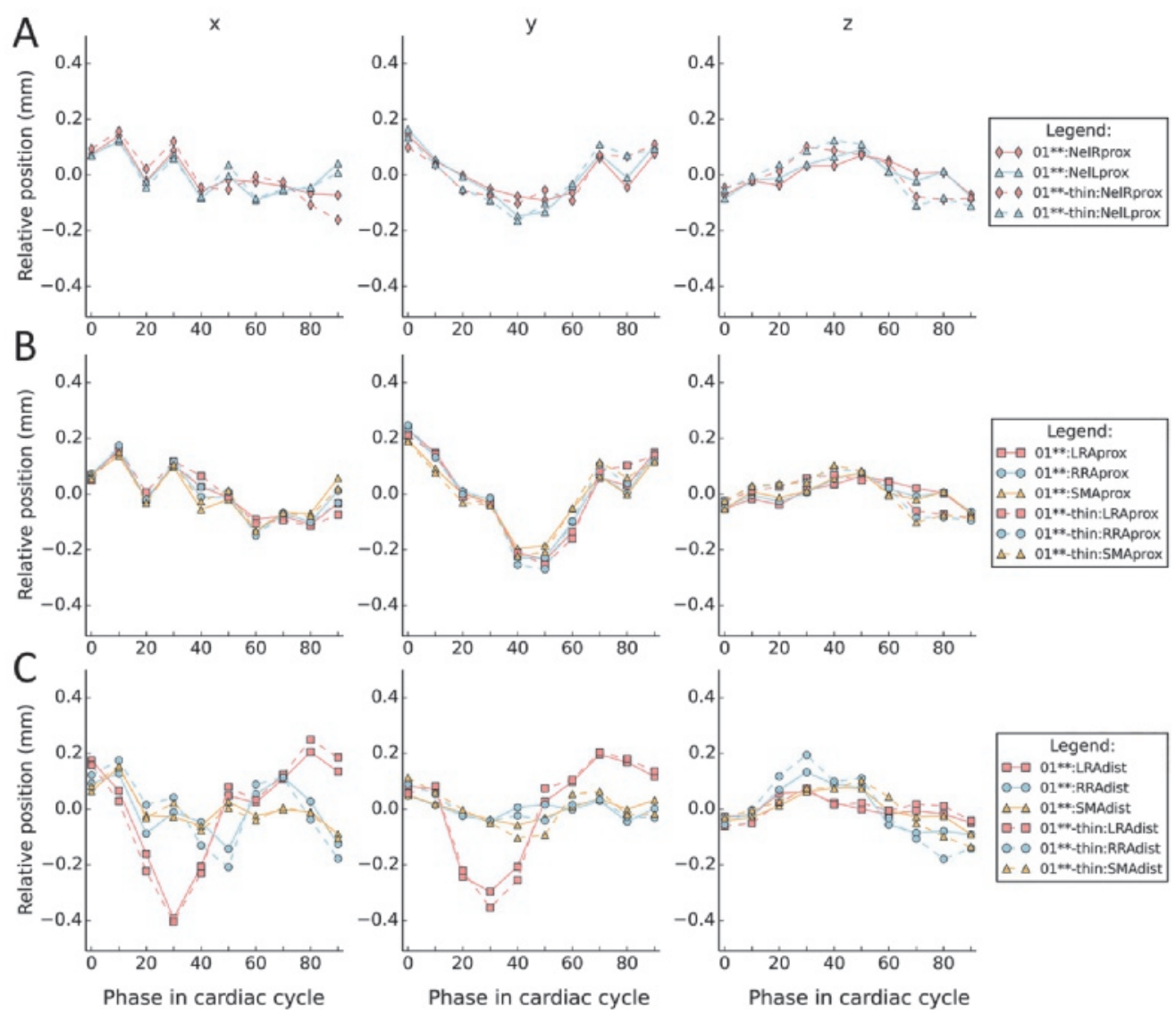

Figure S2. Maximum intensity projections of a single volume at $20 \%$ of the cardiac cycle displaying the volume of the $2.5 \mathrm{~mm}$-slice reconstruction without (A) and with (B) interpolation and the volume of the $0.9 \mathrm{~mm}$-slice reconstruction without interpolation $(\mathrm{C})$. Displacement in $\mathrm{x}-, \mathrm{y}-$, and z-direction of the proximal and distal parts of the Nellix stents and chimney grafts as measured in the 2.5 (solid line) and 0.9 (dashed line) mm-slice reconstruction. LRA, left renal artery; RRA, right renal artery; SMA, superior mesenteric artery; prox, proximal; dist, distal. 

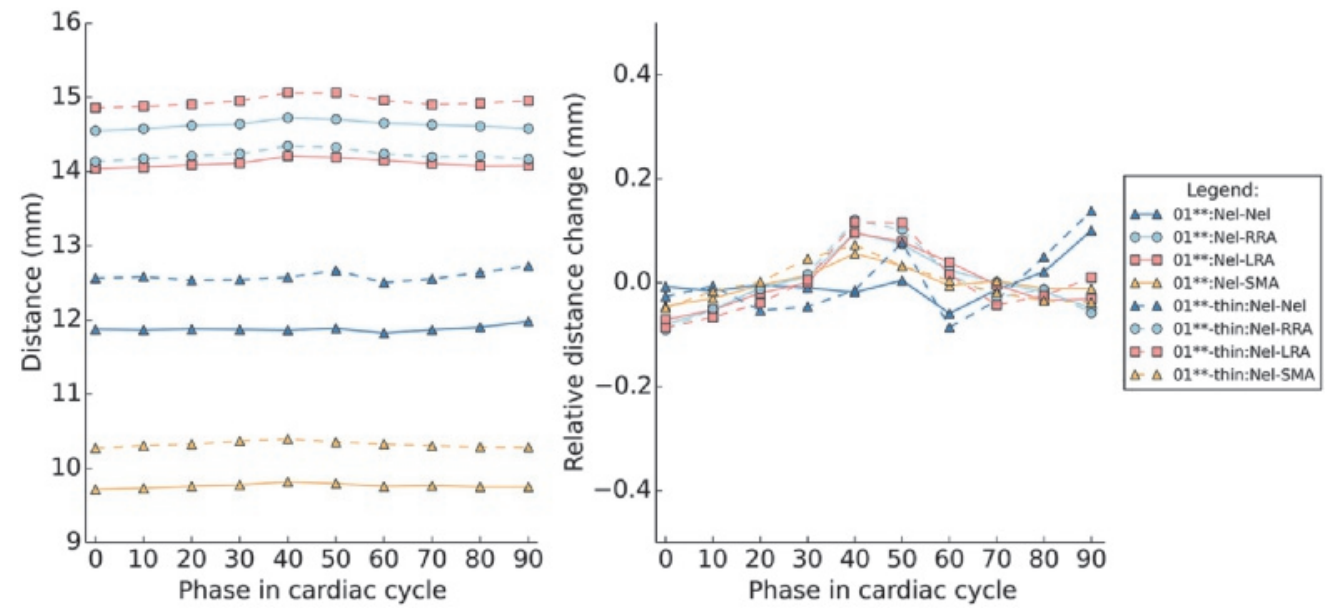

Figure S3. Distance from Nellix-to-Nellix and from Nellix-to-chimney as measured in the 2.5 (solid line) and 0.9 (dashed line) mm-slice reconstruction. LRA, left renal artery; RRA, right renal artery; SMA, superior mesenteric artery; Nel, Nellix stent; Nel-Nel, Nellix-toNellix. 

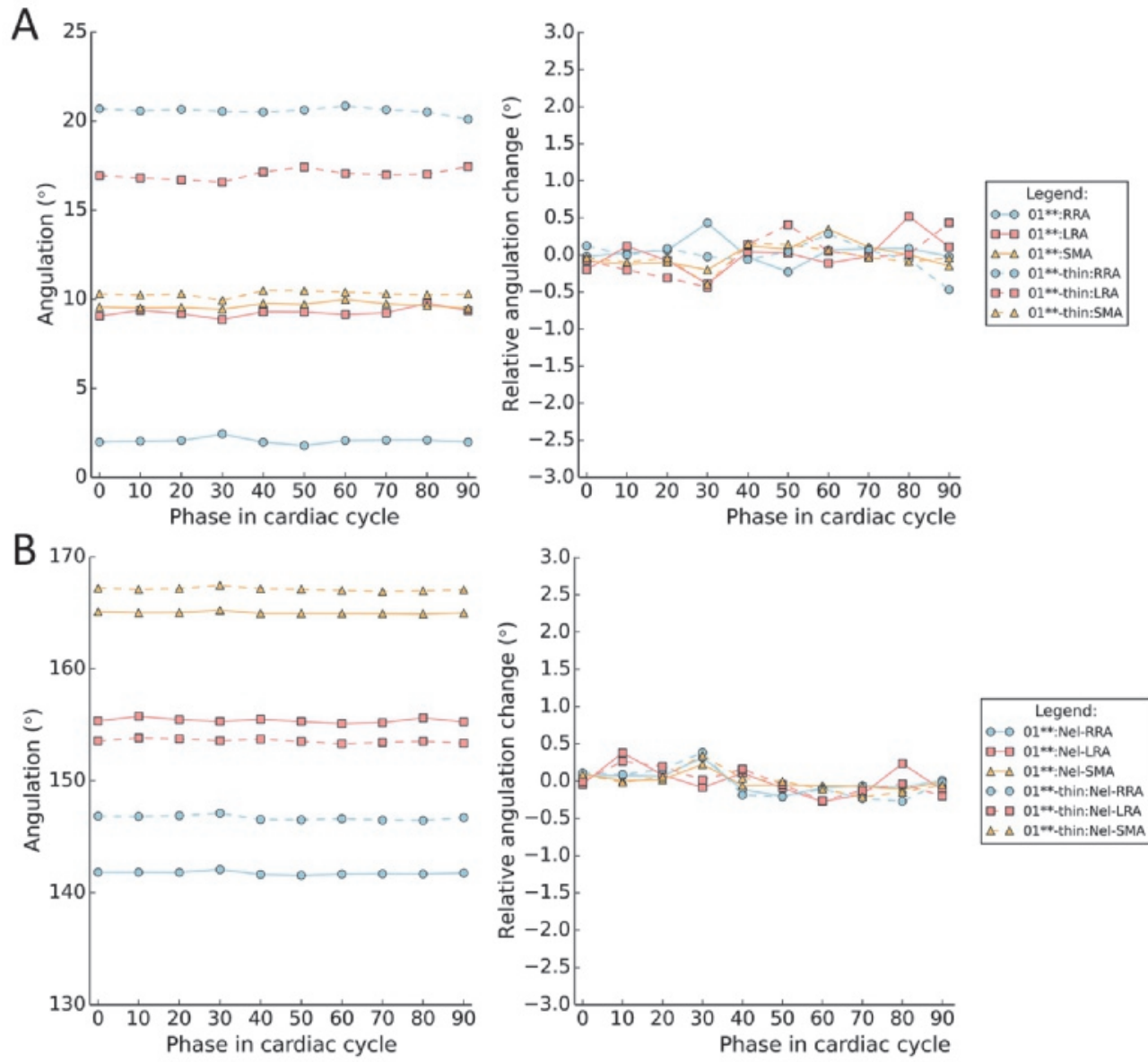

Figure S4. Angles of maximum chimney deflection (A) and Nellix-to-chimney angles are displayed (B) as measured in the 2.5 (solid line) and 0.9 (dashed line) mm-slice reconstruction. LRA, left renal artery; RRA, right renal artery; SMA, superior mesenteric artery; Nel, Nellix stent. 

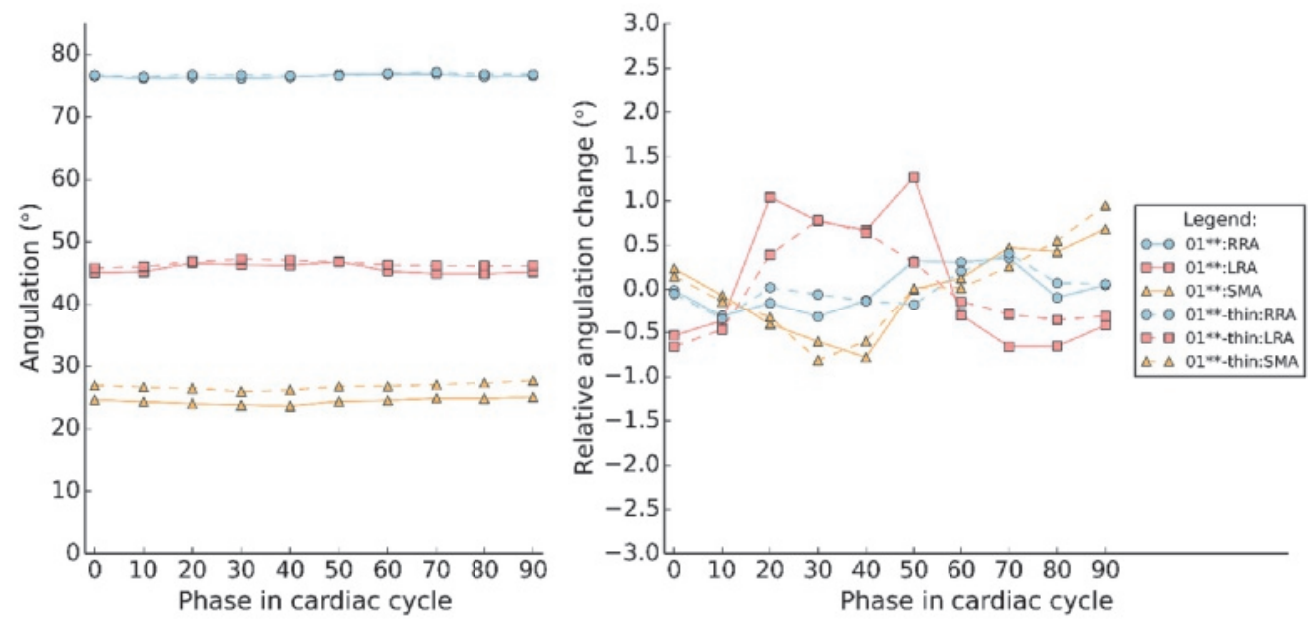

Figure S5. End-stent angles as measured in the 2.5 (solid line) and 0.9 (dashed line) mm-slice reconstruction. LRA, left renal artery; RRA, right renal artery; SMA, superior mesenteric artery. 



$$
\begin{aligned}
& \left.\frac{d x}{d t}=u(x) \cdot t\right) \cdot x^{(0)}=x^{0} \\
& \begin{array}{l}
\frac{d x}{d t}=u(x+t) \\
u(x, t)=U(a(t)
\end{array}
\end{aligned}
$$

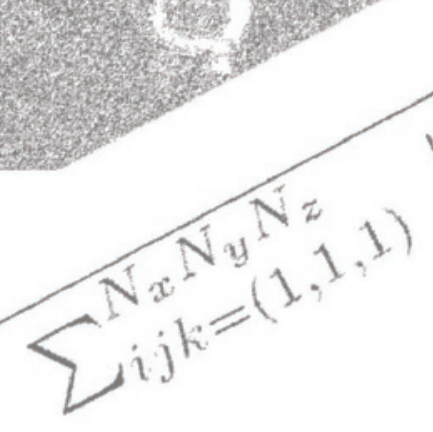

$$
\begin{aligned}
& d_{r} e f \\
& x_{i j} \\
& x+j^{i} \\
& \varepsilon=
\end{aligned}
$$





\title{
Chapter 9
}

\section{Future outlook: A mathematical approach to validate image registration for motion estimation}

\begin{abstract}
Maaike A. Koenrades ${ }^{a, b, c}$, Bernard J. Geurts ${ }^{d}$
${ }^{a}$ Multi-modality Medical Imaging (M3I) group, Faculty of Science and Technology, Technical Medical Centre, University of Twente, Enschede, the Netherlands

${ }^{b}$ Department of Vascular Surgery, Medisch Spectrum Twente, Enschede, the Netherlands

${ }^{c}$ Robotics and Mechatronics (RaM) group, Faculty of Electrical Engineering, Mathematics and Computer Science, Technical Medical Centre, University of Twente, Enschede, the Netherlands

${ }^{d}$ Multiscale Modeling and Simulation, Department of Applied Mathematics, Faculty of Electrical Engineering, Mathematics and Computer Science, Technical Medical Centre, University of, Enschede, the Netherlands
\end{abstract}

A manuscript based on this work is in preparation 


\section{Clinical background}

Knowledge of the in vivo interaction of stent-grafts and native vessels after endovascular aneurysm repair (EVAR) is crucial to design stent-grafts that are both durable and effective to prevent rupture of the aneurysm. Stent-grafts must be able to withstand the in vivo forces imparted by the pulsatile blood flow. However, stent-grafts may alter motion of the aortic vessel wall[1], which could lead to adverse cardiovascular remodeling including stiffening and increased wall stress, potentially resulting in aortic dissection and dilatation[2]. Despite evident improvements in stent-graft design over the past two decades, the reintervention rate of around $10-20 \%[3,4]$ is high and devices still are prone to failure in the long-term[5]. Consequently, a recent expert review underlined the necessity to have an understanding of the human dynamic environment and physiological changes over time in order to improve device designs and avoid complications including endoleaks, device fatigue, and late rupture[6]. Numerical simulations can be useful to study the effect of stent-graft implantation on aortic biomechanics and to predict device failure but to be reliable these methods require realistic boundary conditions that must be obtained from clinical data[7,8]. The introduction of electrocardiogram (ECG)-gated computed tomography (CT) and magnetic resonance imaging (MRI) allows to investigate in vivo motions non-invasively[9]. Still, the measurement of aortic deformation and especially stent-graft deformation during the cardiac cycle is a largely open problem due to the complex 3D motions. Manual tracking of motion and approaches based on 2D slices[10-15] do not suffice to appreciate 3D motions and volumetric changes. Therefore, the development of automated methods for 3D motion tracking is essential. Image registration techniques allow for such automation as they are capable of mapping points from one image to corresponding points in another image[16]. Automated approaches involving image registration techniques have been described in literature[17-23], yet validation is challenging due to a lack of ground truth in clinical image data[16]. In this work, we describe a systematic mathematical approach for the assessment of registration accuracy by transforming real application-specific patient data.

\section{Relation to previous work}

Approaches to measure 3D motion A number of methods has been reported to quantify in 3D aortic lumen displacements[24], radius change[25], longitudinal and circumferential strain[26], and centerline deformation of the aorta[27] and stent-graft[28]. However, these techniques require modeling in each reconstructed phase (volume) in the time-series. This may lead to inconsistencies between models in the different phases resulting in reduced measurement accuracy. Additionally, out of plane movement may not be appreciated[26]. The accuracy of measurements must be sufficient to track motions throughout the time-series volumes. This is particularly important in the abdominal aorta where the motions of interest are small and may approximate the pertinent voxel size[29]. A more appropriate approach is the use of image registration algorithms[30], in particular deformable (non-rigid) registration techniques as aortic deformations do not conform to rigid transformations but may combine radial distension, longitudinal strain and rotation $[27,29]$. 
We have previously used a deformable image registration algorithm[17,31] to study motions of stent-grafts in the abdominal aorta using ECG-gated CT data[32,33] (chapters 5-8). This algorithm employs a groupwise registration approach, similar to Metz et al.[34], to simultaneously register time-resolved volumes to a common average of all volumes, avoiding a bias towards a specific time-point. The registration is related to the well-known Demons algorithm[35] that calculates image forces (e.g., intensities) at the pixels/voxels to drive the registration, resulting in a displacement field that describes the displacement for each pixel/voxel. Different from the Demons algorithm, this algorithm uses the intensities in a less direct manner by taking the gradient magnitude or the absolute of the Laplacian, which makes it more robust for intensity distortions[31,36]. Also, by using a B-spline grid for regularization instead of smoothing the deformation field with a Gaussian, the deformations are constrained to be smooth[34] and physically realistic[37]. The deformation fields can then be applied to the structure of interest obtained by segmentation to estimate its motion. While this registration algorithm may also be applicable to other time-resolved data, including (multimodal) MRI data[31], we focus on CT as it widely available, fast, and it allows for $3 \mathrm{D}$ motion quantification of both the stent-graft (metal frame) and the aorta.

Approaches to validate registration-based techniques Validation of registration algorithms is imperative to ensure reliability of measurements but it is a challenging problem due to a lack of ground truth in clinical image data[16]. Similarity measures such as mutual information, root mean squared error, intensity differences and consistency are often used to evaluate registration performance[30,38] but these measures compare original and deformably registered images without considering a ground truth transformation and hence do not evaluate accuracy by a target registration error[16]. Schwartz et al.[21] registered the aortic wall by model learning methods considering reproducibility of mapping errors for different sets of sample points. Their approach requires segmentation of the aorta at each time point to obtain a set of sample points to learn correspondences. Expert-derived landmark correspondences are widely reported to evaluate registration accuracy[18,39-44]. However, a major drawback is the limited accuracy due to interobserver variabilities. Moreover, distinctive landmarks must be present for the structure of interest and delineation of a sufficient number of landmarks for validation is a tedious and time-consuming task. The use of digital phantoms such as the widely used cardiac-torso (XCAT) phantom[45] provides another means to evaluate registration accuracy. However, these phantoms are application specific and remain a simplified artificial version of the anatomical reality. Additionally, most studies employing a registration algorithm do not perform an additional validation for their specific application[19,46]. The generation of synthetically deformed data provides a known ground truth transformation for validation. Klein[36] and Metz et al.[34] have created synthetically deformed data by applying known deformations to 2D artificial images but these data may not provide sufficient realism. Firouzian et al.[47] created 4D (3D+time) CT data by applying deformation fields found by the registration algorithm in clinical 4D CT scans to a following 3D CT scan of these patients. While this study did make use of real application-specific patient data, this approach may be prone to overestimate the registration accuracy as it uses deformation fields that were created by the algorithm itself. We have 


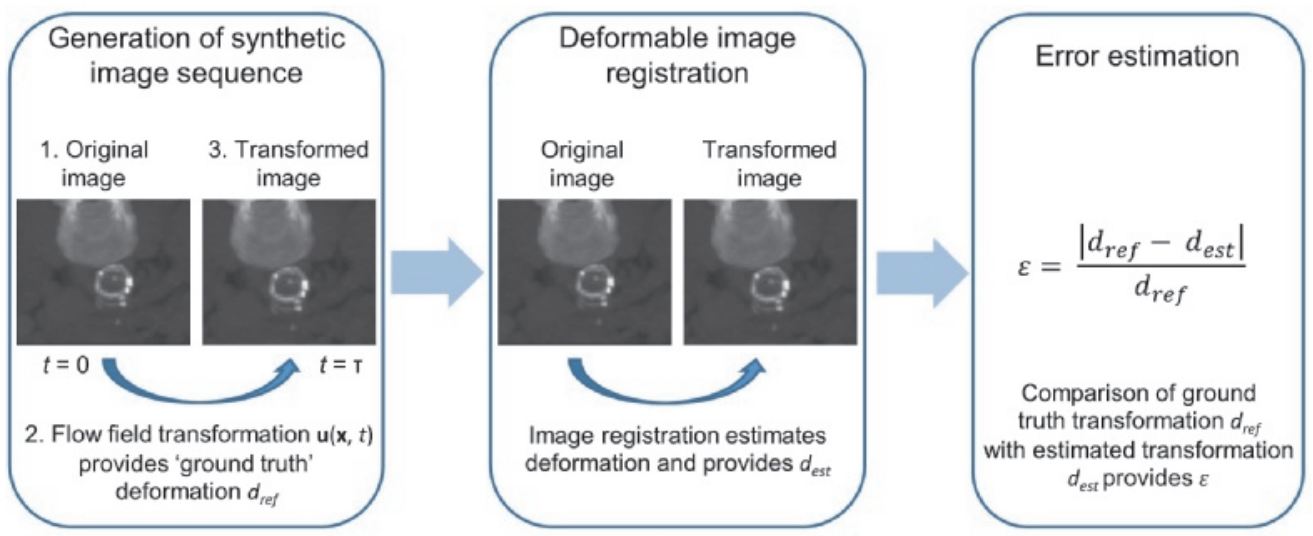

Figure 1. Flowchart of the proposed validation approach. First, the voxels in the original image are moved under a defined flow field $\mathbf{u}(\mathbf{x}, t)$ up to $t$ and interpolated to obtain a deformed image at $t=\tau$. The image registration algorithm then computes the deformation between the original and synthetically transformed image. Comparison of the reference deformation $d_{r e f}$ with the estimated deformation $d_{e s t}$ provides a measure for the registration error $\varepsilon$.

previously performed an experimental validation using a physical anthropomorphic phantom and a motion generating device[32,33] (chapter 6). While this validation showed subvoxel registration accuracy by accurate comparison against known reference motion patterns, incorporating the intrinsic limitations of the CT acquisition itself, the motion patterns were translational only as the physical phantom could not be deformed in a controlled manner to mimic subtle non-rigid transformation. We therefore pursue additional validation of spatial accuracy in silico through the here proposed systematic mathematical approach making use of real application-specific patient data.

Flow field transformation on real-world data to create synthetic images This approach involves the deformation of a given image with a controllable magnitude. We adopt the model of fluid mechanics, in which each voxel of an original image (e.g., volumetric image) is being transported in a prescribed velocity field for some time to yield a deformed image. The 'distance' (displacement vector) between the original and the deformed voxels can be controlled at will, creating a transformed image with a known transformation to test the accuracy of the registration directly with a clearly defined ground truth. The approach is illustrated in Figure 1.

The motion of voxels under an assumed flow field needs further specification. We denote the external 'flow field' by $\mathbf{u}(x, y, z, t)$ depending on position $\mathbf{x}=$ $(x, y, z)$ and time $t$. A volumetric image is composed of 'voxels' with a 'color' $c(x, y, z)$, where the color $c$ takes values between $\left[c_{\min }, c_{\max }\right]$ in some gray scale classification (e.g., Hounsfield unit in CT). The centers of the voxels have a location $\mathbf{x}(t)$ and are assumed to move in the external field $\mathbf{u}$ as test particles, i.e., according to 


$$
\frac{d \boldsymbol{x}}{d t}=\boldsymbol{u}(\boldsymbol{x}(t), t) ; \boldsymbol{x}(0)=\boldsymbol{x}_{0},
$$

This implies that the voxel with color $c\left(\mathbf{x}_{0}\right)$ at time $t=0$ will be located at $\mathbf{x}(t)$ at a later time $t>0$. This provides a simple intuitive model for the deformation of any image

1. obtain the original image with color distribution $c\left(\mathbf{x}_{0}\right)$ on a Cartesian grid of voxels at time $\mathrm{t}=0$

2. move all voxels in the flow field $\mathbf{u}(\mathbf{x}, t)$ up to $t=\tau$

3. interpolate to obtain colors on the Cartesian grid of voxels at $t=\tau$ for the deformed image

In this way we can create any sequence of images and control the deformation. This provides a natural setting for image registration in which the 'ground truth' is explicitly known. Since the deformation can also be made arbitrarily small, also the lower bound on the detectability of deformations can be investigated for any flow field.

Assuming a structured grid of voxels defining an image in $3 \mathrm{D}$ a voxel is a small volume of finite size $\left[x_{i-1 / 2}, x_{i+1 / 2}\right] \times\left[y_{j-1 / 2}, y_{j+1 / 2}\right] \times\left[z_{k-1 / 2}, z_{k+1 / 2}\right]$ with $\left|x_{i+1 / 2}-x_{i-1 / 2}\right|=\Delta$, the size of a voxel. The original color of that voxel is identified as the color in its 'center', i.e., $\left(x_{i}, y_{j}, z_{k}\right)$. The motion of the voxel according to (Equation 1) will start with initial location in this center and carry the voxel to $\mathbf{x}_{i j k}(\tau)$ at time $\tau$. Through interpolation one may then define the 'deformed' image in terms of new voxels that take colors at $\left(x_{i}, y_{j}, z_{k}\right)$ using polynomial interpolation. This procedure can be readily implemented in Matlab (The Mathworks Inc., Natick, MA, USA), exploiting GRIDDATA for convenient interpolation of the displaced voxels at $\mathbf{x}_{i j k}(\tau)$ to a selected grid of voxels.

The main task in this flow analysis is in the definition of a sensible flow field with which original imagery is transformed. For sensitivity analysis one may compose a velocity field in terms of basic, standardized flow patterns such as translation $T$, rotation $R$, stretching $S$, and shearing $S h$. Symbolically, we may express

$$
\boldsymbol{u}(\boldsymbol{x}, t)=U[\alpha(t) T+\beta(t) R+\gamma(t) S+\delta(t) S h]
$$

where $U$ denotes the scale of the flow field and the (time-dependent) weights are denoted by $\alpha, \beta, \gamma$ and $\delta$. Many different velocity fields can be proposed to represent specific aspects of a deforming field. In our application the main components are translation, stretching, and rotation[27,29]. Optimal weights can also be extracted from recordings of a particular patient, creating a one-to-one model for the motion of anatomical structures and medical implants. The actual motion will be over a typical 'distance' of $U \tau$, leading to a natural dimensionless deformation parameter of $D=\mathrm{U}$ $\tau / \Delta$, in terms of the voxel size $\Delta$ in the original image. A deformation parameter $D=n$ then corresponds to a displacement with a scale of $n$ voxel sizes.

To complete this conceptual approach to quantify the spatial accuracy of a particular registration algorithm, a relevant measure of the error needs to be chosen. 
Since we have the actual original and the precise deformed image, we can quantify the deformation as the Euclidean distance between these two, denoted by $d_{\text {ref }}$, i.e.,

$$
d_{\text {ref }}=\sqrt{\left(\frac{1}{N_{x} N_{y} N_{z}} \sum_{i j k=(1,1,1)}^{N_{x} N_{y} N_{z}}\left|\boldsymbol{x}_{i j k}(\tau)-\boldsymbol{x}_{i j k}(0)\right|^{2}\right)}
$$

for an image with $N_{x} \times N_{y} \times N_{z}$ voxels. In addition, a registration algorithm will estimate this Euclidean distance as $d_{e s t}$. The latter depends of course on the selected registration algorithm and the used registration parameters. A convenient, normalized measure for the relative error would then be

$$
\varepsilon=\frac{\left|d_{r e f}-d_{e s t}\right|}{d_{\text {ref }}},
$$

The central question regarding the limits of detectability of differences between images, i.e., the sensitivity of the method, can now be assessed by studying the dependence of $\varepsilon$ on the deformation parameter $D$. This approach can measure which details in a recording can be reliably quantified - one may distinguish measurable motions (e.g., true motion of the aorta or stent-graft) from simple uncertainties.

\section{Discussion}

With the mathematical validation approach proposed here, spatial accuracy and sensitivity of a registration algorithm can be evaluated as we know the ground truth transformation underlying the image sequence in full detail. By transforming original images, we avoid the need to mimic the physics of the image acquisition modality. The output of the proposed approach is a temporal-resolved image sequence similar to the real-world images including the original physical properties and anatomical structures. In order to create an image sequence with multiple time points through a cardiac cycle, the flow patterns can be defined periodically, using weights modelled by combinations of trigonometric functions of time. A similar approach combining realworld data and synthetic periodic deformations was used by Hameeteman et al.[48] to assess the registration accuracy of carotid artery distensibility measurements. However, this study used a scaling factor only (at only one amplitude) to simulate the expansion of a blood vessel while longitudinal displacements and other deformations will also be present in vivo[27,29]. As described, we pursue an implementation that involves multiple standardized flow patterns including translation, rotation, stretching (scaling), and shearing, to either investigate these types of transformations individually or by combining them into one velocity field. Additionally, the approach proposed here is generic and can be applied to clinical images of any modality.

Although this approach allows to evaluate realistic motions, the level of noise and artifacts is the same between image pairs/sequences, which may falsely overestimate algorithm performance[42]. To overcome this issue, image distortions such as Gaussian distributed noise, blurring (Gaussian kernel), and intensity changes may be imposed. Nevertheless, the registration algorithm considered here has been shown to be robust for variations in the intensities between images, noise, and metal artifacts[36]. 
Applying the deformation fields found by the image registration algorithm proposed by Klein et al.[17] as implemented in our previous work[32] to a segmentation of the aortic vasculature allows to investigate aortic pulsatility (e.g., orthogonal to its lumen centerline, longitudinal, rotational, volumetric). This offers the prospect to study device behavior as well as the unstented and stented compliance of vessels for different devices used in EVAR (e.g., abdominal, thoraco-abdominal, thoracic) to both support device development and pre- and post-operative EVAR management.

\section{Acknowledgments}

The authors thank dr. Matthias Schlottbom (Mathematics of Computational Science (MACS) group, Department of Applied Mathematics, University of Twente) for the fruitful discussions.

\section{References}

1. Nauta FJH, de Beaufort HWL, Conti M, Marconi S, Kamman A V., Ferrara A, et al. Impact of thoracic endovascular aortic repair on radial strain in an ex vivo porcine model. Eur J Cardio-Thoracic Surg. 2017;51:ezw393.

2. Raaz U, Zöllner AM, Schellinger IN, Toh R, Nakagami F, Brandt M, et al. Segmental aortic stiffening contributes to experimental abdominal aortic aneurysm development. Circulation. 2015;131:1783-95.

3. De Bruin JL, Baas AF, Buth J, Prinssen M, Verhoeven ELG, Cuypers PWM, et al. Longterm outcome of open or endovascular repair of abdominal aortic aneurysm. $\mathrm{N}$ Engl $\mathrm{J}$ Med. 2010;362:1881-9.

4. Jonker LT, de Niet A, Reijnen MMPJ, Tielliu IFJ, Zeebregts CJ. Mid- and Long-Term Outcome of Currently Available Endografts for the Treatment of Infrarenal Abdominal Aortic Aneurysm. Surg Technol Int. 2018;33:239-50.

5. Patel R, Sweeting MJ, Powell JT, Greenhalgh RM. Endovascular versus open repair of abdominal aortic aneurysm in 15-years' follow-up of the UK endovascular aneurysm repair trial 1 (EVAR trial 1): a randomised controlled trial. Lancet. 2016;388:2366-74.

6. Belvroy VM, Houben IB, Trimarchi S, Patel HJ, Moll FL, Van Herwaarden JA. Identifying and addressing the limitations of EVAR technology. Expert Rev Med Devices. 2018;15:541-54. 7. Chuter TAM. Durability of Endovascular Infrarenal Aneurysm Repair: When Does Late Failure Occur and Why? Semin Vasc Surg. 2009;22:102-10.

8. Beebe HG. Lessons learned from aortic aneurysm stent graft failure; observations from several perspectives. Semin Vasc Surg. 2003;16:129-38.

9. Laskowski I, Verhagen HJM, Gagne PJ, Moll FL, Muhs BE. Current state of dynamic imaging in endovascular aortic aneurysm repair. J Endovasc Ther. 2007;14:807-12.

10. van Keulen JW, van Prehn J, Prokop M, Moll FL, van Herwaarden JA. Dynamics of the Aorta Before and After Endovascular Aneurysm Repair: A Systematic Review. Eur J Vasc Endovasc Surg. 2009;38:586-96.

11. van Keulen JW, Vincken KL, van Prehn J, Tolenaar JL, Bartels LW, Viergever MA, et al. The Influence of Different Types of Stent Grafts on Aneurysm Neck Dynamics after Endovascular Aneurysm Repair. Eur J Vasc Endovasc Surg. 2010;39:193-9.

12. Pol JA, Truijers M, van der Vliet JA, Fillinger MF, Marra SP, Renema WKJ, et al. Impact of dynamic computed tomographic angiography on endograft sizing for endovascular aneurysm repair. J Endovasc Ther. 2009;16:546-51.

13. Lalande A, Khau van Kien P, Salvé N, Ben Salem D, Legrand L, Walker P, et al. Automatic Determination of Aortic Compliance With Cine-Magnetic Resonance Imaging. Invest Radiol. 
2002;37:685-91.

14. Krug R, Boese JM, Schad LR. Determination of aortic compliance from magnetic resonance images using an automatic active contour model. Phys Med Biol. 2003;48:2391-404.

15. Jackson CE, Shirodaria CC, Lee JMS, Francis JM, Choudhury RP, Channon KM, et al. Reproducibility and accuracy of automated measurement for dynamic arterial lumen area by cardiovascular magnetic resonance. Int J Cardiovasc Imaging. 2009;25:797-808.

16. Liu Z, Deng X, Wang G. Accuracy Validation for Medical Image Registration Algorithms: a Review. Chinese Med Sci J. 2012;27:176-81.

17. Klein A, Renema W, Vliet JA, Oostveen LJ, Hoogeveen Y, Schultze Kool LJ, et al. Motion Calculations on Stent Grafts in AAA. In: Grundmann RT, editor. Diagnosis, Screening and Treatment of Abdominal, Thoracoabdominal and Thoracic Aortic Aneurysms. [Rijeka]: InTechOpen; 2011. p. $125-44$.

18. Biesdorf A, Wörz S, Müller T, Weber TF, Heye T, Hosch W, et al. Model-based segmentation and motion analysis of the thoracic aorta from 4D ECG-gated CTA images. Med Image Comput Comput Interv. 2011;14:589-96.

19. Nasr B, Le Ven F, Savean J, Ben Salem D, Nonent M, Gouny P, et al. Characterization of the Physiological Displacement of the Aortic Arch Using Non-Rigid Registration and MR Imaging. Eur J Vasc Endovasc Surg. 2017;53:282-9.

20. Satriano A, Rivolo S, Martufi G, Finol EA, Di Martino ES. In vivo strain assessment of the abdominal aortic aneurysm. J Biomech. 2015;48:354-60.

21. Schwartz E, Langs G, Holfeld J, Gottardi R, Loewe C, Peloschek P, et al. Segmentation and Deformation Analysis of the Aorta in Gated CTA sequences in a MDL Framework. Med Image Underst Anal. 2009;13:27-32.

22. Schwartz E, Gottardi R, Holfeld J, Loewe C, Czerny M, Langs G. Evaluating deformation patterns of the thoracic aorta in gated cta sequences. Proc IEEE Int Symp Biomed Imaging. 2010;21-4.

23. Langs G, Paragios N, Desgranges P, Rahmouni A, Kobeiter H. Learning deformation and structure simultaneously: In situ endograft deformation analysis. Med Image Anal. 2011;15:12-21.

24. Piccinelli M, Vergara C, Antiga L, Forzenigo L, Biondetti P, Domanin M. Impact of hemodynamics on lumen boundary displacements in abdominal aortic aneurysms by means of dynamic computed tomography and computational fluid dynamics. Biomech Model Mechanobiol. 2013;12:1263-76.

25. Wentz R, Manduca A, Fletcher JG, Siddiki H, Shields RC, Vrtiska T, et al. Automatic segmentation and co-registration of gated CT angiography datasets: measuring abdominal aortic pulsatility. Proc SPIE Med Imaging. 2007;6511:65111I-9.

26. de Beaufort HWL, Nauta FJH, Conti M, Cellitti E, Trentin C, Faggiano E, et al. Extensibility and Distensibility of the Thoracic Aorta in Patients with Aneurysm. Eur J Vasc Endovasc Surg. 2017;53:199-205.

27. Choi G, Cheng CP, Wilson NM, Taylor CA. Methods for quantifying three-dimensional deformation of arteries due to pulsatile and nonpulsatile forces: Implications for the design of stents and stent grafts. Ann Biomed Eng. 2009;37:14-33.

28. Itoga NK, Suh G-Y, Cheng CP. Stabilization of the Abdominal Aorta During the Cardiac Cycle with the Sac-Anchoring Nellix Device. Ann Vasc Surg. 2018;52:312.e7-312.e12.

29. Flora HS, Woodhouse N, Robson S, Adiseshiah M. Micromovements at the aortic aneurysm neck measured during open surgery with close-range photogrammetry: Implications for aortic endografts. J Endovasc Ther. 2001;8:511-20.

30. Alam F, Ur Rahman S, Ullah S, Khalil A, Uddin A. A Review on Extrinsic Registration Methods for Medical Images. Tech J Univ Eng Technol Taxila. 2016;21:110-9.

31. Klein A, Kroon D-J, Hoogeveen Y, Schultze Kool LJ, Renema WKJ, Slump CH. Multimodal image registration by edge attraction and regularization using a B-spline grid. Proc SPIE Med Imaging. 2011;7962:796220-8.

32. Koenrades MA, Struijs EM, Klein A, Kuipers H, Reijnen MMPJ, Slump CH, et al. Quantitative 
Stent Graft Motion in ECG Gated CT by Image Registration and Segmentation: In Vitro Validation and Preliminary Clinical Results. Eur J Vasc Endovasc Surg. 2019; [Epub ahead of print].

33. Koenrades MA, Struijs EM, Klein A, Kuipers H, Geelkerken RH, Slump CH. Validation of an image registration and segmentation method to measure stent graft motion on ECG-gated CT using a physical dynamic stent graft model. Proc SPIE Med Imaging. 2017;10134:1013411-8.

34. Metz CT, Klein S, Schaap M, van Walsum T, Niessen WJ. Nonrigid registration of dynamic medical imaging data using $\mathrm{nD}+\mathrm{t} \mathrm{B}$-splines and a groupwise optimization approach. Med Image Anal. 2011;15:238-49.

35. Thirion J-P. Image matching as a diffusion process: an analogy with Maxwell's demons. Med Image Anal. 1998;2:243-60.

36. Klein A. A tool for studying the motion of stent grafts in AAA. Segmentation and motion estimation of stent grafts in abdominal aortic aneurysms. [Enschede]: PhD Dissertation, University of Twente; 2011. p. 121-37.

37. Noblet V, Heinrich C, Heitz F, Armspach J-P. Retrospective evaluation of a topology preserving non-rigid registration method. Med Image Anal. 2006;10:366-84.

38. Varadhan R, Karangelis G, Krishnan K, Hui S. A framework for deformable image registration validation in radiotherapy clinical applications. J Appl Clin Med Phys. 2013;14:4066.

39. Castillo R, Castillo E, Guerra R, Johnson VE, McPhail T, Garg AK, et al. A framework for evaluation of deformable image registration spatial accuracy using large landmark point sets. Phys Med Biol. 2009;54:1849-70.

40. Vandemeulebroucke J, Sarrut D, Clarysse P. The POPI model, a pointvalidated pixel-based breathing thorax model. Proc XVth Int Conf Use Comput Radiat Ther. 2007;195-9. Available: http://www.creatis.insa-lyon.fr/rio/popi-model.

41. Vaman C, Staub D, Williamson J, Murphy MJ. A method to map errors in the deformable registration of 4DCT images. Med Phys. 2010;37:5765-76.

42. Latifi K, Zhang G, Stawicki M, van Elmpt W, Dekker A, Forster K. Validation of three deformable image registration algorithms for the thorax. J Appl Clin Med Phys. 2013;14:3834.

43. Gu X, Pan H, Liang Y, Castillo R, Yang D, Choi D, et al. Implementation and evaluation of various demons deformable image registration algorithms on a GPU. Phys Med Biol. 2010;55:207-19.

44. Sarrut D, Boldea V, Miguet S, Ginestet C. Simulation of four-dimensional CT images from deformable registration between inhale and exhale breath-hold CT scans. Med Phys. 2006;33:605-17.

45. Segars WP, Sturgeon G, Mendonca S, Grimes J, Tsui BMW. 4D XCAT phantom for multimodality imaging research. Med Phys. 2010;37:4902-15.

46. Schwartz E, Holfeld J, Czerny M, Loewe C, Langs G. Towards Predicting the Effects of Stent-Grafting on the Motion of the Thoracic Aorta. MICCAI Proc. 2012;17-24.

47. Firouzian A, Manniesing R, Metz CT, Risselada R, Klein S, van Kooten F, et al. Quantification of Intracranial Aneurysm Morphodynamics from ECG-gated CT Angiography. Acad Radiol. $2013 ; 20: 52-8$.

48. Hameeteman K, Rozie S, Metz CT, Manniesing R, van Walsum T, van der Lugt A, et al. Automatic carotid artery distensibility measurements from CTA using nonrigid registration. Med Image Anal. 2013;17:515-24. 



\section{Chapter 10}

\section{General discussion}

In this chapter, the research described in this thesis is placed in the context of endovascular aneurysm repair (EVAR) and the implications of our findings are discussed from a clinicians' and engineers' perspective. Finally, future perspectives for this line of research are presented and concluding remarks are made.

This thesis aims to broaden our understanding of stent-graft devices' in-vivo behavior to provide insight that can support both physicians and device manufactures in their efforts to maximize the durability of EVAR. Meticulous image processing methods involving image registration, model-based stent-graft segmentation, and centerline extraction were developed, validated, and applied. We have investigated in detail the longitudinal and cardiac-pulsatility induced behavior of an infrarenal fixating stent-graft with a nitinol self-expanding stent-ring design. Through the establishment of a prospective study - Longitudinal Study of Pulsatility and Expansion in Aortic Stent-grafts (LSPEAS; Trialregister.nl identifier NTR4276) - we gathered electrocardiogram (ECG)-gated computed tomography (CT) data of eventually 15 patients through a careful study protocol with a 2-years follow-up period. Predominantly, we have focused on the proximal double stent-ring in the aortic neck; the factor known to be crucial for durable exclusion of the aneurysm[1]. Secondly, the stent-graft's limb configuration was investigated to help better understand the emergence of limb occlusion. Furthermore, the proximal stability of a sac-anchoring endosystem in combination with chimney grafts (chEVAS) was evaluated during the cardiac cycle using 11 retrospectively gathered ECG-gated CT angiography scans.

\section{What have we learned?}

\section{Part I: Geometric changes over time}

With EVAR, the primary concern of the physician is to achieve adequate proximal seal and positional stability. For self-expanding stent-grafts, adequate sizing and conformation of the stent-graft to the aortic neck anatomy are critical to maintain graft-wall contact and prevent leakage of blood flow into the aneurysm sac. Additionally, dilatation of the aortic neck can result in loss of contact between the vessel wall and the stent-graft. It is therefore useful to understand the evolution of stent-graft 
expansion and the response of the aortic neck. In chapter 2, we have learned that the proximal double sealing ring of the Anaconda stent-graft (Terumo Aortic, Inchinnan, Scotland, UK) expanded to near nominal size (first ring, 97\%, second ring 95\%, on average) within 6 months after EVAR and primarily within the first month, while a broad range of initial oversizing was applied (17-47\%). Consequently, to monitor neck dilation, baseline CT scans should be taken within 1 month after EVAR, preferably at discharge. Additionally, degenerative neck dilation should be monitored when the rings have expanded to their nominal size. The observation of some initially highly asymmetrically shaped rings showed that the stent-rings can conform to the aortic neck. Yet, during expansion all rings became nearly circular, implying eventual remodeling of the neck to the nominal size of the rings. Significant wall stress due to ring expansion could cause intimal hyperplasia and lead to degeneration of the wall. In our practice, oversizing was regularly increased $(>20 \%)$ to enlarge the adaptive capacity of the sealing rings should the aortic neck dilate. Based on our observations in chapter 2, we currently advise to avoid oversizing outside the IFU (10-20\%). To prevent full stent-ring expansion, one may argue to decrease the radial force of the stent-rings by design modification. Yet, decreasing the radial force (e.g., less wire windings to form the stent-ring) may increase the extent of radial expansion and contraction due to pulsatile blood flow, which from an engineers' point of view can be considered cumbersome for device durability (e.g., device fatigue). Nevertheless, engineers may use these insights to model stent-ring expansion and fatigue under various conditions considering factors such as radial strength, oversizing, and vessel compliance in patient specific geometries.

While neck dilation due to stent-ring expansion may raise concerns for proximal stability, a localized dilatation does not necessarily result in dilatation of the entire neck. If below the sealing rings no dilation occurs, downward displacement will be averted by the imposed conical shape of the neck. Actually, it was observed in chapter 2 that the first stent-ring expanded further than the second stent-ring, which also imposes a conical shape by the extent of ring expansion. The sealing rings were designed as such that the first stent-ring has a larger radial force compared to the second stent-ring (by the number of wire windings). In chapter 3, we continue our analysis of the remodeling of the aortic neck above, at, and below the two fixation and sealing rings. It was shown that in most patients the neck diameters below the stentring zone remained unchanged or even decreased over time whereas diameter increase was related to downstream device displacement. This implies that some patients may require less frequent follow-up while others may be at risk to develop device migration and endoleak. Early expansion of the stent-rings to their nominal size could be a warning sign for the development of dilatation, indicating a mismatch between the compliance of the vessel and the radial force of the device. In line with chapter 2 , these observations suggest to avoid excessive oversizing. Additionally, they advocate a sophisticated individual preoperative assessment of the wall characteristics to tailor treatment strategies. For example by quantifying pulsatile distension in ECG-gated CT to allow for adequate sizing and device selection (e.g., more/less compliant) and to identify patients with (non)compliant vessel walls that should be monitored more frequently (perceived risk of dilatation, migration, endoleak, and/or fatigue).

Since high placement of a stent-graft in the aortic neck may benefit sealing length 
and positional stability[2,3], we went on to investigate the effect of saddle alignment on the position of the Anaconda sealing rings in the neck (chapter 4). Unfolding of the saddle-shape had resulted in downward displacement of its peaks and no upward displacement of its valleys in cases with moderate oversizing. We further show that the length of stent-graft device within the neck is reduced by downward movement of the peaks and by a decrease in tilt, leaving a proximal portion of the neck unused, which may be unfavorable in the long term. In summary, our results show that it is feasible to position the peaks pararenally with the valleys juxtarenally $(\geq 3 \mathrm{~mm}$ below the renal artery ostia) without compromising the renal arteries. Nevertheless, the safety and efficacy of juxtarenal valley positioning should be further investigated in a clinical registry. Also, positioning the peaks pararenally instead of infrarenally could subject the stent-ring to more stressful flow patterns and forces imposed by the blood flow of which the consequences for device durability are unclear.

In chapter 5, we have assessed changes in the geometry of the Anaconda stentgraft limbs after EVAR. Over time, we observe an increase in curvature, shortening of the limbs, and a corresponding decrease in distance between successive stent-rings, particularly in two patients that later on were diagnosed with limb occlusion. These geometric changes can result in inwards folding of the graft fabric, which is known to contribute to embolization and thrombosis[4-6]. This implies that such changes in the geometry may help to predict limb occlusion and thus could support patientspecific follow-up schemes. Besides prediction of failure, these insights may aid in optimization of the limb design with respect to the frequency or shape of the rings in the limbs. Although the O-shaped stent-rings have an evident benefit in tortuous iliac anatomy, a more M-shaped or saddle-shaped ring structure can reduce inwards folding of the fabric. The effect of various designs on fabric folding and flow patterns may be investigated through computational fluid dynamics or empirically with high-framerate contrast-enhanced ultrasound particle image velocimetry in vitro and potentially in vivo[7,8].

\section{Part II: Cardiac cycle related behavior}

Considering that a stent-graft is implanted in a dynamic endovascular environment, it seems injudicious to base EVAR management on static CT imaging (c.q. the gold standard). As more and more (younger) patients are treated using increasingly complex device configurations (e.g., fenestrated and branched stent-grafts, sac-anchoring endosystems, and combinations using chimney grafts), device durability certainly remains a concern. Pulsatile blood flow subjects an implanted device to roughly 40 million cycles per year, which challenges fixation and sealing but also the mechanical stability of the device (e.g., component detachment, fatigue-induced fracture of a stent wire)[9-12]. Needless to say, the development of methods to measure in vivo the dynamic stent-artery interaction is imperative to develop preclinical tests that suit in vivo conditions and to allow for prediction of failure in clinical use. In chapter 6 , we have implemented a previously proposed algorithm[13-15] to evaluate the accuracy of stent-graft motion quantification in time-resolved CT data (e.g., ECG-gated CT). This methodology combines image registration and segmentation techniques to allow for 3-dimensional tracking of motion. The uttermost error threshold of $0.3 \mathrm{~mm}$ for 
the amplitude of motion was experimentally derived using a physical phantom[16,17]. Application of the algorithm to clinical ECG-gated CT data demonstrated the relevance of the method for different stent-graft designs including Z- and O-shaped stents, fenestrated devices, and sac-anchoring devices combined with chimney grafts. These data demonstrate that the degree of motion in $\mathrm{x}-, \mathrm{y}-$, and $\mathrm{z}$-direction may differ per fixation site and between devices, which should be incorporated in device design and durability tests. This can avoid failure modes such as fracture of visceral stents[18].

In chapter 7 , we have applied the algorithm to evaluate the cardiac pulsatilityinduced motion and deformation of the Anaconda stent-graft sealing rings during follow-up. Throughout the follow-up period, we observe limited radial expansion and contraction during the cardiac cycle $(<3.5 \%)$, which is beneficial for proximal stability and device durability. Yet reduced aortic compliance is less favorable towards the risk of atherosclerotic events[19]. Our observations imply that the radial force imposed by the sealing rings was adequate to counteract aortic pulsatility to prevent metal fatigue in the long term. Still, the stent-rings expanded asymmetrically and assessment of cyclic change in curvature identified regions that are subject to reduced fatigue safety factors under in-vivo loading conditions, and are therefore of particular importance when considering device durability. Engineers can use these insights to validate their fatigue simulations (e.g., by comparing estimated levels of pulsatility and compliance or stress distribution) or even to determine the internal forces induced in the stent wires (i.e., stress) based on inversed modeling simulations (e.g., imposing positional change of points on the stents instead of defining external forces/flow acting on it). Consequently, one could determine whether and where fracture would occur given a certain number of cycles. Fortunately, no cases of fracture of Anaconda sealing rings have been reported in the treatment of infrarenal abdominal aortic aneurysm (AAA). Still, a case of fracture in a fenestrated Anaconda device 3 years after treatment of a juxtarenal AAA has recently been observed, though with prosperous aneurysm shrinkage[20]. Investigation of this fracture case by Terumo Aortic identified post-implant disease progression as well as calcification in the vicinity of the stent. These, together with a greater degree of stent-ring motion for fenestrated Anaconda compared with the infrarenal Anaconda (postulated from observations of fenestrated Anaconda pulsatility[16]), may have caused unusually high localized loading of the stent-ring for this particular case. The ability to measure localized stent-ring curvature changes, as per the novel methodology described in chapter 7, could provide a very useful tool for such stent fracture investigations where post-implant ECG-gated CT data is available. Results of an investigator initiated observational study (LSPEAS-fenestrated; Trialregister.nl identifier NTR6225) are awaited to gain insight in the pulsatility-related motions in fenestrated Anaconda devices.

Endovascular aneurysm sealing (EVAS) is still in an evolutionary phase, which mandates increasing knowledge to further develop this technique [21]. Moreover, combined with chimney grafts to treat para- and juxtarenal abdominal aortic aneurysms, this technique may be liable for component instability due to forces imposed by pulsatile blood flow. In chapter 8, we evaluated the proximal stability of the Nellix EndoVascular Aneurysm Sealing System (Endologix, Irvine, CA, USA) in combination with chimney grafts during the cardiac cycle. This study demonstrates minimal cyclical changes in device configuration as seen by at most $0.4 \mathrm{~mm}$ change in distance 
between device components, $2.6^{\circ}$ change in chimney graft angle, $1.7^{\circ}$ change in angle between chimney graft and EVAS stent, and $3.3^{\circ}$ change in end-stent branch angle. Even though these observations seem favorable to maintain component stability during the cardiac cycle, they do not predict the likelihood of future migration, which appears to be the most common complication after EVAS[21,22]. The observed cyclical displacement of the Nellix stents in longitudinal and lateral direction can be used in preclinical tests to design a device that can better endure pulsatile drag forces, for example numerical simulation of pulsatile drag forces. Perhaps a new generation sac-anchoring device should comprise additional active fixation (e.g., barbs/anchors into the polymer). In addition, the parameters considered in this work may be important to monitor since over time the developing drag forces by the blood flow may alter the motion of device components, potentially resulting in migration and/or endoleak. Nevertheless, life-long yearly surveillance by ECG-gated CT will rapidly accumulate radiation exposure. Still, associated radiation risks for CT surveillance are much smaller than AAA-related risks[23]. Following a traditional surveillance protocol (first year: $87.5 \mathrm{mSv} / \mathrm{y}$, second year: $35 \mathrm{mSv} / \mathrm{y}$, and subsequent years 17.5 $\mathrm{mSv} / \mathrm{y})$, comparable to ECG-gated CT surveillance in terms of dose, Geleijns et al[23] reported 8 radiation induced deaths vs. 91 AAA-related deaths per 1000 patients that underwent EVAR at 65 years of age. It applies to any device that radiation exposure related risks must be weighed against AAA-related risks.

\section{Future perspectives}

The long-term objective of this line of research is to develop a clinical tool that can be used by clinicians and device manufacturers to evaluate the behavior of implanted stent-grafts for the benefit of clinical decision making and device design. As reflected by the wide variety of stent-graft failures, the endovascular environment and responses of stent-grafts and native vessels are presently not well enough understood to create preclinical tests that accurately simulate in vivo conditions. With the introduction of new and modified devices in particular it is highly relevant to characterize in vivo motions and geometric changes that occur over time to verify and customize the preclinical tests to the situation in vivo. For example, numerical simulations driven by device-specific in vivo behavior may help to identify vulnerabilities of a stentgraft design. This can result in device re-design to minimize failure modes in future patients and avoid failure on a large scale. Moreover, regulatory bodies may soon require information on device durability that was based on dynamics measured in vivo (e.g, in post-market evaluations).

Prediction of failure was not investigated in this thesis. However, once a substantial number of patients has been treated and carefully evaluated by ECG-gated CT for several years, it should become possible to distinguish 'natural' behavior from behavior predisposing to failure (static and dynamic factors). Thereafter, the clinical use of ECG-gated CT during the first postoperative year (e.g., predischarge, 3 months, 12 months) may already allow to differentiate between high- and low-risk patients and thus for patient specific follow-up (e.g., frequency, CT/duplex ultrasound).

Additionally, insight in the stented and unstented vessel pulsatile behavior is relevant for device design and potentially for the prediction of failure. Also, it may 
support planning and sizing. Incorporating patient-specific anatomy and aortic dynamics in a computer model could help to select the most appropriate device for a patient. While the work described in this thesis did not involve such evaluation, the methodology as applied in chapters 5 to 8 can certainly be applied to aortic vessels, enabling radial, longitudinal and volumetric quantifications. Still, such application necessitates additional validation as discussed in chapter 9.

Finally, endovascular devices for the pararenal aorta, the descending thoracic aortic and the aortic arch are expected to continue to be developed and broadened in utilization the coming years[24,25]. The methods presented in this thesis support these ongoing developments with an expected unique patient-device interaction for each route.

\section{Concluding remarks}

The developed and applied methods enabled us to visualize and quantify in vivo stent-graft response and improve our understanding of the ongoing interplay of the device and the vessel. The work presented in this thesis contributes to the foundation of informed shared decision making in device selection, sizing, positioning, and surveillance, and the prediction of failure in an early stage. Moreover, this work supports the development of sound preclinical tests to introduce EVAR devices that well fit and can endure the endovascular environment. The presented methods can be readily utilized for different endovascular devices, including devices with branches, fenestrations, and chimney grafts. 


\section{References}

1. de Vries JPPM. The Proximal Neck: The Remaining Barrier to a Complete EVAR World. Semin Vasc Surg. 2012;25:182-6.

2. Schuurmann RCL, van Noort K, Overeem SP, van Veen R, Ouriel K, Jordan WD, et al. Determination of Endograft Apposition, Position, and Expansion in the Aortic Neck Predicts Type Ia Endoleak and Migration After Endovascular Aneurysm Repair. J Endovasc Ther. 2018;25:366-75.

3. Zarins CK, Bloch DA, Crabtree T, Matsumoto AH, White RA, Fogarty TJ, et al. Stent graft migration after endovascular aneurysm repair: Importance of proximal fixation. J Vasc Surg. 2003;38:1264-72.

4. Mantas GK, Antonopoulos CN, Sfyroeras GS, Moulakakis KG, Kakisis JD, Mylonas SN, et al. Factors predisposing to endograft limb occlusion after endovascular aortic repair. Eur J Vasc Endovasc Surg. 2015;49:39-44.

5. Di Achille P, Tellides G, Figueroa CA, Humphrey JD. A haemodynamic predictor of intraluminal thrombus formation in abdominal aortic aneurysms. Proc R Soc A Math Phys Eng Sci. 2014;470:20140163.

6. Georgakarakos E, Argyriou C, Schoretsanitis N, Ioannou C V, Kontopodis N, Morgan R, et al. Geometrical Factors Influencing the Hemodynamic Behavior of the AAA Stent Grafts: Essentials for the Clinician. Cardiovasc Intervent Radiol. 2014;37:1420-9.

7. Voorneveld J, Engelhard S, Vos HJ, Reijnen MMPJ, Gijsen F, Versluis M, et al. High-FrameRate Contrast-Enhanced Ultrasound for Velocimetry in the Human Abdominal Aorta. IEEE Trans Ultrason Ferroelectr Freq Control. 2018;65:2245-54.

8. Hoving AM, de Vries EE, Voorneveld J, Groot Jebbink E, Mikhal J, Versluis M, et al. In vitro high-frame-rate contrast-enhanced ultrasound particle image velocimetry in a carotid artery stent. Proc SPIE Med Imaging. 2018;10580:5.

9. Raghavan ML, Kratzberg JA, Golzarian J. Introduction to biomechanics related to endovascular repair of abdominal aortic aneurysm. Tech Vasc Interv Radiol. 2005;8:50-5.

10. Jacobs TS, Won J, Gravereaux EC, Faries PL, Morrissey N, Teodorescu VJ, et al. Mechanical failure of prosthetic human implants: A 10-year experience with aortic stent graft devices. J Vasc Surg. 2003;37:16-26.

11. Beebe HG. Lessons learned from aortic aneurysm stent graft failure; observations from several perspectives. Semin Vasc Surg. 2003;16:129-38.

12. Chuter TAM. Durability of Endovascular Infrarenal Aneurysm Repair: When Does Late Failure Occur and Why? Semin Vasc Surg. 2009;22:102-10.

13. Klein A, van der Vliet JA, Oostveen LJ, Hoogeveen Y, Schultze Kool LJ, Renema WKJ, et al. Automatic segmentation of the wire frame of stent grafts from CT data. Med Image Anal. 2012;16:127-39.

14. Klein A, Renema W, Vliet JA, Oostveen LJ, Hoogeveen Y, Schultze Kool LJ, et al. Motion Calculations on Stent Grafts in AAA. In: Grundmann RT, editor. Diagnosis, Screening and Treatment of Abdominal, Thoracoabdominal and Thoracic Aortic Aneurysms. [Rijeka]: InTechOpen; 2011. p. $125-44$

15. Klein A. A tool for studying the motion of stent grafts in AAA. Segmentation and motion estimation of stent grafts in abdominal aortic aneurysms. [Enschede]: PhD Dissertation, University of Twente; 2011. p. 121-37.

16. Koenrades MA, Struijs EM, Klein A, Kuipers H, Reijnen MMPJ, Slump CH, et al. Quantitative Stent Graft Motion in ECG Gated CT by Image Registration and Segmentation: In Vitro Validation and Preliminary Clinical Results. Eur J Vasc Endovasc Surg. 2019; [Epub ahead of print].

17. Koenrades MA, Struijs EM, Klein A, Kuipers H, Geelkerken RH, Slump CH. Validation of an image registration and segmentation method to measure stent graft motion on ECG-gated CT using a physical dynamic stent graft model. Proc SPIE Med Imaging. 2017;10134:1013411-8.

18. Verhoeven ELG, Vourliotakis G, Bos WTGJ, Tielliu IFJ, Zeebregts CJ, Prins TR, et al. Fenestrated Stent Grafting for Short-necked and Juxtarenal Abdominal Aortic Aneurysm: An 8-Year 
Single-centre Experience. Eur J Vasc Endovasc Surg. 2010;39:529-36.

19. Oliver JJ, Webb DJ. Noninvasive Assessment of Arterial Stiffness and Risk of Atherosclerotic Events. Arterioscler Thromb Vasc Biol. 2003;23:554-66.

20. Tielliu I, Zeebregts C. Fractured Proximal Nitinol Ring in a Fenestrated Anaconda Device. Eur J Vasc Endovasc Surg. 2019;57:553.

21. Reijnen MMPJ, Holden A. Status of Endovascular Aneurysm Sealing After 5 Years of Commercial Use. J Endovasc Ther. 2018;25:201-6.

22. England A, Torella F, Fisher RK, McWilliams RG. Migration of the Nellix endoprosthesis. J Vasc Surg. 2016;64:306-12.

23. Geleijns J, Koning O, van Bockel H. SU-FF-I-169: Endovascular Abdominal Aortic Aneurysm Repair: Patient Dose and Radiation Risks. Med Phys. 2009;36:2474.

24. Buck DB, van Herwaarden JA, Schermerhorn ML, Moll FL. Endovascular treatment of abdominal aortic aneurysms. Nat Rev Cardiol. 2014;11:112-23.

25. Jackson BM, Carpenter JP. Devices Used for Endovascular Aneurysm Repair: Past, Present, and Future. J Vasc Surg. 2009;19104:39-43. 




\section{Summary (Dutch)}

Een aneurysma is een uitpuiling of opzwelling in de wand van een bloedvat. Meestal ontstaat een aneurysma als gevolg van slagaderverkalking die de wand van het bloedvat verzwakt. Als de verwijding zich bevindt in het in de buik gelegen gedeelte van de aorta, wordt dit een aneurysma aorta abdominalis (AAA) genoemd. In de loop der tijd verliest de vaatwand zijn elasticiteit waardoor de vaatwand kan scheuren door de kracht van een normale bloeddruk in het aneurysma.

In verschillende onderzoeken is aangetoond dat het uitschakelen van het aneurysma door het plaatsen van een endoprothese (een kunststof vaatprothese met metalen stents) via de lies (endovasculair aorta reparatie) op korte termijn betere resultaten geeft dan de conventionele en belastende open chirurgische methode. Echter, op lange termijn hebben 10-20\% van de patiënten een reinterventie nodig door falen van de endoprothese (scheuren van de vaatwand, lekkage van bloed langs de endoprothese in het aneurysma, of het afsluiten van bloedstroom door de endoprothese). Falen kan onder andere gerelateerd zijn aan structurele schade aan de endoprothese, verwijding van de aorta door progressie van de ziekte, en het losraken van de endoprothese van de vaatwand onder invloed van het pulserende bloedvat.

Om de lange termijn uitkomsten te verbeteren is meer inzicht nodig in het gedrag van geïmplanteerde endoprothesen, om de best passende endoprothese te kunnen kiezen voor een patiënt en om het ontwerp van endoprothesen te verbeteren. In dit proefschrift zijn onderzoeksmethoden ontwikkeld, gevalideerd en toegepast om de voortgaande interactie tussen endoprothese en bloedvat te onderzoeken op basis van hartslag gekoppelde computer tomografie (CT) scans.

In dit proefschrift is hoofdzakelijk onderzoek gedaan naar een patiënt cohort dat behandeld is met een Anaconda AAA endoprothese en tot 2 jaar na interventie is gevolgd met hartslag gekoppelde CT scans. In het eerste deel van het proefschrift zijn veranderingen in geometrie van de endoprothese en de aorta onderzocht gedurende follow-up. Het tweede deel richt zich op bewegingen van de endoprothese die ontstaan tijdens de hartslag. Hierin is ook onderzoek gedaan naar bewegingen van een ander type endoprothese (Nellix endoprothese) die gebruik maakt van een polymeer zak om het aneurysma in zijn geheel af te sluiten van de bloedstroom (endovasculair aneurysma sealing). 


\section{Deel 1: Veranderingen in geometrie na verloop van tijd}

De Anaconda endoprothese heeft 2 Nitinol stent ringen bovenin de endoprothese die zich ontvouwen als een zadelvorm na ontplooiing van de endoprothese via de lies. Doordat de diameter van de ringen groter gekozen wordt dan de diameter van de aorta ('oversizing'), drukken de ringen tegen de vaatwand waarmee het aneurysma uitgeschakeld wordt van de bloedstroom ('sealing'). In hoofdstuk 2 is onderzocht in hoeverre de diameter van de ringen uitzet gedurende de periode na implantatie van de endoprothese. Gedurende de $1^{e}$ maand vonden reeds grote veranderingen plaats waarna binnen 6 maanden de ringen waren uitgezet tot nabij de nominale ring diameter ( $1^{e}$ ring $97 \%, 2^{e}$ ring $95 \%$ ). Dit betekent dat de mate van oversizing gevolgen heeft voor de verwijding van de aorta ter hoogte van de ringen. Verder is geobserveerd dat de ringen zich asymmetrisch kunnen vormen naar de vorm van de aorta maar dat uiteindelijk de ringen weer een ronde vorm aannemen.

In hoofdstuk 3 is onderzocht of het uitzetten van de ringen ook leidt tot het verwijden van de aorta aangrenzend aan de ringen. Uit deze studie is gebleken dat onder de stent ringen de aorta diameter stabiel bleef of af nam in de meeste patiënten waar een toename in diameter werd gezien in gevallen waar de endoprothese zich naar beneden verplaatste. Boven de stent ringen bleef de aorta diameter onveranderd. Deze bevindingen impliceren dat bepaalde patiënten mogelijk minder follow-up nodig hebben waar anderen een groter risico lopen op falen en dus nauwlettender gevolgd zouden moeten worden.

De verandering van de zadelvorm door het uitzetten van de ringen heeft mogelijk ook gevolgen voor de positie van de ringen in de aorta ten opzichte van de nierslagaders. Bij implantatie worden de ringen ontplooid net onder de aftakking van de nierslagaders. Door de zadelvorm is het echter mogelijk om de ringen met de pieken van de zadelvorm ter hoogte van de nierslagaders te plaatsen terwijl de dalen van de zadelvorm zich onder de nierslagaders bevinden en de nierslagaders daarmee niet worden afgesloten van bloedstroom. In hoofdstuk 4 is de verplaatsing van de pieken en dalen van de zadelvorm onderzocht. Voornamelijk de pieken bleken omlaag te verplaatsen bij het afvlakken van de zadelvorm. Ook kwamen schuin geplaatste ringen (onder een hoek ten opzichte van de as van de aorta) meer recht te liggen. De dalen kwamen met maximaal $3 \mathrm{~mm}$ omhoog alleen in gevallen waar veel oversizing was toegepast ( $>30 \%$ t.o.v. de aorta diameter). Deze bevindingen suggereren dat het afvlakken van de zadelvorm geen risico vormt voor het afsluiten van de nierslagaders, waarbij een plaatsing van de dalen aanliggend aan de nierslagaders een veilige techniek is indien gematigde oversizing wordt toegepast (10-20\%).

Hoofdstuk 5 richt zich op de 2 pootjes van de Anaconda endoprothese, welke voorbij het aneurysma een stukje doorlopen in de arteria iliaca communis (gemeenschappelijke heupslagader). Thrombus (bloedstolsel) vorming in de pootjes kan leiden tot het afsluiten van de bloedstroom naar de bil en benen waardoor reinterventie nodig is. De pootjes bestaan uit kunststof met ongeveer elke $5 \mathrm{~mm}$ een stent ring. Veranderingen in de configuratie van de pootjes kunnen leiden tot het naar binnen vouwen van de kunststof en daarmee mogelijk bijdragen aan thrombus vorming. Gedurende follow-up zijn een toename in curvatuur, een afname in poot lengte en een afname in afstand tussen de ringen in de pootjes geobserveerd. Deze inzichten kunnen bijdragen 
aan het voorspellen van poot afsluiting en het verbeteren van het ontwerp van de endoprothese.

\section{Deel 2: Hartcyclus gerelateerd gedrag}

Er is weinig bekend over het dynamische gedrag van geïmplanteerde endoprothesen, hoewel dit van groot belang is voor het ontwikkelen van duurzame endoprothesen. De bloedstroom induceert zo'n 40 miljoen cycli per jaar waaraan de endoprotheseaorta combinatie wordt blootgesteld. Hiermee wordt de positiestabiliteit van de endoprothese en de sealing op de proef gesteld maar ook de mechanische stabiliteit van de endoprothese.

In hoofdstuk 6 hebben we een eerder ontwikkeld algoritme geïmplementeerd om de nauwkeurigheid van geanalyseerde endoprothese bewegingen in hartslag gekoppelde CT scans te evalueren. Deze methode combineert beeldregistratie en segmentatie technieken. Een fantoom opstelling met een gecontroleerd bewegende endoprothese werd gebruikt. De grootst bepaalde fout op de verplaatsing was $0.3 \mathrm{~mm}$. De analyse methode werd verder succesvol toegepast op 4 klinische casussen met elk een ander type endoprothese.

In hoofdstuk 7 is deze methode toegepast om de beweging en vervorming van de 2 Anaconda sealing ringen te onderzoeken tijdens de hartslag. Een radiale expansie en contractie van de ringen van $<3.5 \%$ diameter verandering werd vastgesteld, wat gunstig is voor de positiestabiliteit en het risico op metaalmoeheid reduceert. De mogelijke gevolgen van het dempen van het natuurlijk pulseren van de aorta zijn echter onduidelijk. De methode zoals beschreven in deze studie maakt het mogelijk om locaties op de stents te identificeren die het meest vatbaar zijn voor dynamische spanning door het cyclisch buigen. Dit werk biedt een basis voor verbeterde spanningsrek analyses, duurzaamheidstests en ontwerp verificatie.

In hoofdstuk 8 zijn hartslag gerelateerde bewegingen van de Nellix endoprothese onderzocht om inzicht te krijgen in de stabiliteit van de configuratie waarin naast de 2 standaard parallelle stents ook stents werden geplaatst in de nier- en/of darmslagaders. Bewegingen tijdens de hartslag waren minimaal. De afstand tussen endoprothese onderdelen veranderde ten hoogste $0.4 \mathrm{~mm}$, de buiging van de stents in de nier/darmslagaders was ten hoogste $2.6^{\circ}$ en de hoek tussen de endoprothese onderdelen veranderde ten hoogste $1.7^{\circ}$. Deze observaties demonstreren een stabiele situatie tijdens de hartslag. Desalniettemin zijn de parameters zoals gemeten in deze studie relevant voor follow-up. Na verloop van tijd kunnen bewegingen van endoprothese onderdelen veranderen onder invloed van trekkrachten door de bloedstroom, wat kan leiden tot neerwaartse verschuiving en lekkage van bloed langs de polymeer zak, de meest voorkomende complicatie bij endovasculair aneurysma sealing.

Het ontwikkelen van kwantitatieve methoden vraagt ook om evaluatie en validatie van de methode voor de beoogde toepassing. Het valideren van een registratie algoritme is ingewikkeld doordat in de beelddata de daadwerkelijke bewegingen niet bekend zijn. De fantoom experimenten zoals toegepast in hoofdstuk 6 zijn erg waardevol voor het bepalen van de nauwkeurigheid en gevoeligheid van de meetmethode maar zijn tegelijk ook een vereenvoudiging van de werkelijke complexe bewegingen die in het lichaam plaatsvinden. In hoofdstuk 9 wordt een wiskundige aanpak beschreven om de 
nauwkeurigheid van beeldregistratie te evalueren door klinische beelddata kunstmatig te vervormen.

\section{Slotopmerkingen}

De methoden zoals beschreven in dit proefschrift maken het mogelijk om endoprothese-aorta interactie te volgen gedurende follow-up op basis van hartslag gekoppelde CT scans. De inzichten verkregen in dit proefschrift dragen bij aan beter geïnformeerde klinische besluitvorming omtrent endoprothese selectie, positionering en controles. Daarnaast kunnen de verkregen inzichten leiden tot aanpassingen aan het ontwerp van endoprothesen waardoor in de toekomst de kans op complicaties zou kunnen verminderen. Dit werk ondersteunt het ontwikkelen van realistischere preklinische tests en het ontwikkelen van duurzame endoprothesen. 


\section{List of Publications}

\section{Peer reviewed publications}

Koenrades M.A., Bosscher M.R.F., Ubbink J.T, Slump C.H., Geelkerken R.H. Geometric remodeling of the perirenal aortic neck at and adjacent to the double sealing ring of the Anaconda stent-graft after endovascular aneurysm repair. J Endovasc Ther. 2019. In press.

Koenrades M.A., Struijs E.M., Klein A., Kuipers H., Reijnen M.M.P.J., Slump C.H., Geelkerken R.H. Quantitative stent-graft motion in ECG-gated CT by image registration and segmentation: In vitro validation and preliminary clinical results. Eur J Vasc Endovasc Surg. 2019. [Epub ahead of print].

Koenrades M.A., Klein A., Leferink A.M, Slump C.H., Geelkerken R.H. Evolution of the proximal sealing rings after endovascular aortic aneurysm repair using the Anaconda AAA stent graft. J Endovasc Ther. 2018;25:480-491

Woliner-van der Weg, W., Deden, L.N., Meeuwis, A.P.W., Koenrades, M.A., Peeters, L.H.C., Kuipers, H., Laanstra, G.J., Gotthardt, M., Slump, C.H., Visser, E.P. A 3Dprinted anatomical pancreas and kidney phantom for optimizing SPECT/CT reconstruction settings in beta cell imaging using ${ }^{111}$ In-exendin. EJNMMI Phys. 2016;3:29.

Leferink, A.M., Fratila, R.M., Koenrades, M.A., van Blitterswijk, C.A., Velders, A., Moroni, L. An open source image processing method to quantitatively assess tissue growth after non-invasive magnetic resonance imaging in human bone marrow stromal cell seeded 3D polymeric scaffolds. PLoS ONE. 2014;9:e115000.

\section{Peer reviewed in preparation}

Koenrades M.A., Slump C.H., Geelkerken R.H. Electrocardiogram gated computed tomographic angiography quantification of sealing ring dynamics in patients treated with an Anaconda stent-graft. In preparation.

Koenrades M.A., Geurts B.J. A mathematical approach to validate image registration for motion estimation. In preparation.

J.A. Simmering*, M.A. Koenrades*, C.H. Slump, R.H. Geelkerken. Geometric changes in Anaconda endograft limbs after endovascular aneurysm repair: A potential predictor for limb occlusion. 2019. Submitted. *Shared first authorship. 
Koenrades M.A., Donselaar E.J., van Erp M.A.J.M., Loonen T.G.J., van Lochem P., Klein A., Slump C.H., Geelkerken R.H., Reijnen M.M.P.J. Dynamic computed tomography angiography analysis of cardiac-pulsatility induced motion and deformation after endovascular aneurysm sealing with chimney grafts. 2019. Submitted.

Koenrades M.A., Simmering J.A., Slump C.H., Geelkerken R.H. Peak and valley alignment of the Anaconda proximal saddle-shaped rings after endovascular aortic aneurysm repair: Implications for device positioning and sealing length. 2019. Submitted.

\section{Conference proceedings}

Koenrades M.A., Struijs E.M., Klein A., Kuipers H., Geelkerken R.H., Slump C.H. Validation of an image registration and segmentation method to measure stent graft motion on ECG-gated CT using a physical dynamic stent graft model. Proc SPIE Med Imaging. 2017;10134:1013411-8.

\section{Oral presentations}

Longitudinal study of pulsatility and expansion for Anaconda stents: Analysis of invivo behavior from ECG-gated CT. Terumo Aortic academic research collaborations conference, Glasgow, Scotland. September 2019.

Interaction of the endograft and the infrarenal neck after EVAR with the Anaconda stent-graft. Leipzig Interventional Course (LINC), Leipzig, Germany. January 2019.

Validation of an image registration and segmentation method to measure stent graft motion on ECG-gated CT using a physical dynamic stent graft model. SPIE Medical Imaging Conference, in Computer-Aided Diagnosis, Orlando, Florida, USA. February 2017.

Anaconda stent graft in short infrarenal necks: From imaging to clinical results. Leipzig Interventional Course (LINC), Leipzig, Germany. January 2016.

Dynamics of the proximal fixation and sealing rings. Post academic training "Vascular \& Cardiovascular Experiences and Expertise", Arnhem, the Netherlands. September 2015 .

\section{Poster presentations}

Peak and valley alignment of the Anaconda proximal saddle-shaped rings after endovascular aortic aneurysm repair: Implications for device positioning. International annual meeting of the European Society for Vascular Surgery (ESVS), Valencia, Spain. September 2018. [Awarded 1st prize for best poster presentation]

Adaptation of the peri-renal aorta after placement of an Anaconda AAA stent graft. Leipzig Interventional Course (LINC), Leipzig, Germany. February 2018. 


\section{Biography}

Maaike Anne Koenrades was born May 9, 1989 in Purmerend, the Netherlands. In 2007 she graduated from pre-university education at the Da Vinci College PSG in Purmerend. Thereafter, she studied Technical Medicine at the University of Twente, from which she obtained her MSc. degree Cum Laude in 2014. During her studies, Maaike worked as a student employee at the Experimental Centre for Technical Medicine (ECTM) between 2008 and 2012 and she was a student representative in the education committee of Technical Medicine from 2008 to 2011. Furthermore, she co-organized the 2010 edition of the Great Dutch Student Championship (GNSK) between 2009 and 2010 as a treasurer and commissioner of logistics.

Maaike performed her graduation project enti-

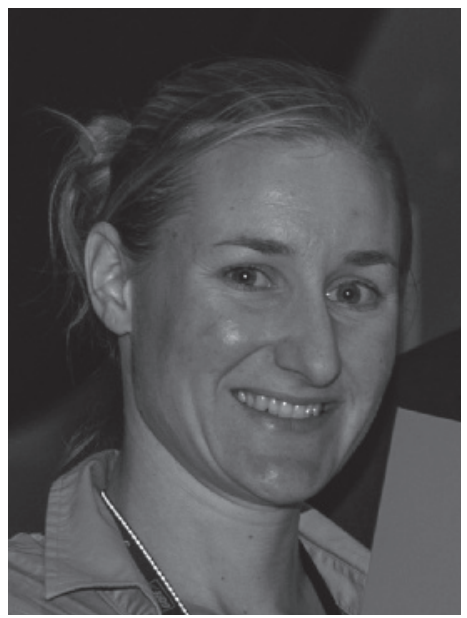
tled 'Longitudinal study of pulsatility and expansion in abdominal aortic stent grafts' at the department of surgery, Medisch Spectrum Twente, Enschede, the Netherlands, and the Robotics and Mechatronics group, University of Twente. In 2014, based on this graduation work on in vivo analysis of implanted stent-grafts, she obtained an individual grant from the Twente Graduate School (TGS) for a 6 months Jr. Researcher position to obtain external funding for a PhD trajectory. In 2015, she was awarded the TGS award for best research proposal on which a research grant was obtained from Vascutek Terumo, presently trading as Terumo Aortic.

Maaike continued her $\mathrm{PhD}$ in the Robotics and Mechatronics group under supervision of prof.dr.ir. Cornelis H. (Kees) Slump and from 2018 in the newly formed Multimodality Medical Imaging (M3I) group under supervision of prof.dr. Robert H. (Bob) Geelkerken. Her PhD research was focused on the development, validation, and application of modern image processing methods to broaden our understanding of in-vivo stent-graft behavior for the benefit of clinical decision making and device design. Her work contributes to the foundation of future studies in abdominal, thoraco-abdominal, and thoracic stent grafting, which will be initiated in the ADEAR (Aortic Dynamics after Endovascular Aneurysm Repair) program 2019-2023, co-funded by Terumo Aortic and Health Holland. 



\section{Dankwoord}

Lieve familie, vrienden, collega's en allen die betrokken zijn geweest bij mijn promotietraject, ter afsluiting een woord van dank.

Allereerst Bob, je hebt vanaf mijn afstuderen een begeleidende rol gehad in het onderzoekspad dat we zijn gaan bewandelen. Je hebt mij veel geleerd en laten zien van de klinische praktijk en mij ondersteund in het beschouwen van vraagstukken vanuit het perspectief van de vaatchirurg. Dit heeft mij geholpen om te komen tot de onderzoeken zoals beschreven in dit proefschrift. Gaandeweg ben je klinisch hoogleraar geworden en ook mijn promotor, waarmee je met nog meer toewijding en enthousiasme dit project hebt ondersteund. Ik heb genoten van de verschillende buitenland bezoeken samen in Glasgow en Santa Rosa die mede dankzij jou mogelijk zijn gemaakt. Ik ben blij dat het onderzoek een vervolg krijgt en denk dat hiervoor nu een mooie basis ligt. Heel veel succes!

Kees, ook jij bent al sinds mijn master opdracht betrokken geweest als begeleider. Naast je drukke onderwijsagenda ben je altijd betrokken gebleven bij dit onderzoek. Bedankt voor je hulp in het weerleggen van lastige reviewer vragen en je relativerende kijk op zaken waardoor ik weer moed had om verder te puzzelen.

Bernard, toen ik op zoek was naar een wiskundige die mij wellicht kon helpen met het manipuleren van medische beelden was jij bereid te helpen. We zijn nog niet gekomen tot een volwaardig wetenschappelijk artikel maar de basis is beschreven en alle handvaten zijn nu ontwikkeld voor het uitvoeren van de experimenten. Bedankt voor je inzet. Bedankt ook voor je betrokkenheid in de laatste fase door mij te ondersteunen met het proeflezen van verschillende hoofdstukken.

Almar, na jouw $\mathrm{PhD}$ ben je altijd zijdelings betrokken gebleven bij dit onderzoeksproject. Bedankt voor het delen van je Python kennis en de bijdragen die je hebt geleverd in de verschillende hoofdstukken. Het was erg fijn af en toe met jou te kunnen werken!

Remke, bedankt voor je ondersteuning in de eerste fasen van dit onderzoeksproject om de juridische en administratieve zaken met betrekking tot de financiering rond te krijgen. 
RaM, met mijn onderzoeklijn was ik binnen RaM wellicht 'een aparte' maar ik heb mij altijd zeer welkom gevoeld. Bedankt voor alle gezellige momenten, het faciliteren van lab opstellingen en de attente bloemetjes als ik of Anne weer eens geblesseerd waren. Stefano, bedankt dat ik mijn onderzoek heb mogen uitvoeren binnen jouw groep.

Beste kantoorgenoten, Best office, een naam die al aangeeft dat gezelligheid op ons kantoor wel aanwezig was. Dank daarvoor. Het was niet altijd makkelijk om mij weg te trekken achter mijn computer maar de strakke koffietiming van Frieda hielp hier enorm bij en ook het tikken van kopjes was een duidelijk teken dat het tijd was voor een refill. De rumoer werd mij soms wel wat veel waar jullie ook vast om hebben kunnen lachen als ik weer met oordoppen en gehoorbeschermers achter mijn computer zat. Marcel, dank voor het altijd maar regelen van de zaalvoetbalwedstrijdjes en Rob voor het initiëren van vele activiteiten.

Frieda, Stefan, na zo veel lief en leed met jullie gedeeld te hebben gedurende mijn $\mathrm{PhD}$ ben ik blij dat jullie mijn paranimfen wilden zijn. Bedankt voor jullie support en uiteraard voor alle koffie, lunch en borrel momenten met heerlijke-diepgaande-zinloze gesprekken, en voor Stefan ook voor de MTB momenten om even uit te waaien.

M3i, erg fijn dat ik ook bij jullie welkom ben geheten en dat ik aan de start heb mogen staan van deze nieuwe vakgroep. De vakgroepuitjes en borrels hebben snel gezorgd voor een goede groepsvorming waarna onder de hoede van Srirang nu flink gebouwd wordt aan een voltallige onderzoeksgroep. Erik GJ, Erik K, Jaimy, Stefan, Bryan, Marije, Lennart, bedankt voor het proeflezen van mijn thesis en het stellen van kritische vragen. Richte, dank voor het beschikbaar stellen van een template van je proefschrift ter inspiratie.

Jaimy, na veel M2 stagiaires (bedankt voor jullie bijdragen!) was jij de eerste M3-er die ik heb mogen begeleiden. Vanuit je afstudeeropdracht heb je het vraagstuk rondom pootocclusie van de Anaconda endoprothese verder opgepakt. Bedankt voor je bijdrage en je inzet waardoor we zijn gekomen tot een wetenschappelijk artikel. Succes met de vele vervolgonderzoeken!

MST, vaatchirurgie, vaatchirurgen, AIOS, radiologie, laboranten, dank voor jullie ondersteuning van de klinische trial vanuit de radiologie en heelkunde. Bert en Anja, jullie bijdragen waren onmisbaar voor het opzetten en goed uitvoeren van de $\operatorname{LSPEAS}(\mathrm{F})$ trial(s). Dank.

Terumo Aortic, Robbie, David, Vincent, Craig, and others who have been involved. It has been a pleasure to discuss the ongoing research in this project. This has helped me to consider things from an engineers' point of view. TA, I would like to thank you for the confidence to support this research and the appreciation of my work. I am glad that TA will continue to support the research conducted by our group, of which I am sure will lead to more useful insights for device design. John, bedankt 
voor je verbindende rol tussen onze onderzoeksgroep en TA in Glasgow. Dank ook voor het meerijden naar verschillende congresbezoeken.

Voetbal vrienden, (oud) Drienerlo, Loesjes, Richtersbleek dames, om maar een paar van jullie te noemen, Annemarie, Sandra, Marlien, Vera B, Ines, Vera V, Leonie, Inge, Jolanda, Claudia, Anne, Mariel, Marieke, Rita, Brigitte, Monique; samen voetballen, wintersporten, borrelen, het heeft gezorgd voor de vele ontspanmomenten die ik nodig had. Dank jullie wel!

TG chicks, Lidy, Anniek, Marit, Laura P, Laura D, Nicole, Annika; ook jullie hebben gezorgd voor leuke weekendjes weg en etentjes. Daarnaast was het fijn om een aantal van jullie al te zien promoveren. Een beetje afkijken kan geen kwaad ;)

Lieve buurtjes, Marieke, Mo, Jasmien, Noura, zo vaak als ik knuffels (oké en koffie en maaltijden) heb gehad van jullie die mij weer nieuwe energie gaven, zo veel waardeer ik jullie en ben ik jullie dankbaar. Hopelijk treffen jullie mij voortaan minder aan achter een laptop nu deze laatste PhD loodjes voltrokken zijn.

Buurtjes Hans en Ans, ook jullie mogen niet ontbreken in dit dankwoord. Ik vond het leuk dat jullie graag op de hoogte wilden blijven. Hans, dank voor je ondersteuning in menige klus rond het huis en dank aan jullie beiden voor de gezellige buurtmomenten.

Lieve ouders, jullie hebben mij altijd gesteund en stonden open voor alles dat ik wilde ondernemen. Dat waardeer ik enorm, eveneens de interesse die jullie altijd tonen in wat er reilt en zeilt in mijn en ons leven.

Lieve Anne, de laatste loodjes voor ons beiden in dezelfde periode, gecombineerd met een badkamerverbouwing, was misschien niet de meest optimaal geplande situatie. Gelukkig hebben we al heel wat meegemaakt samen en komen we ook hier zeker doorheen. Je doet het ongelofelijk goed in deze laatste periode van de zwangerschap. Petje af. Heel veel dank voor je inhoudelijke bijdragen gedurende mijn PhD, voor alle spar momenten en je mentale steun. Jij wees me op de TGS grant wat de deur heeft geopend voor mijn promotietraject. Op de momenten waar het moeilijk werd heb je mij weer verder geholpen door samen een nieuw plan te maken. Ik ben je ontzettend dankbaar, geniet dagelijks van je aanwezigheid (zelfs tijdens je zwangerschap;) en heb veel zin in ons leven 'na de PhDs', samen met ons kleintje!

Dank jullie! Hora est! 




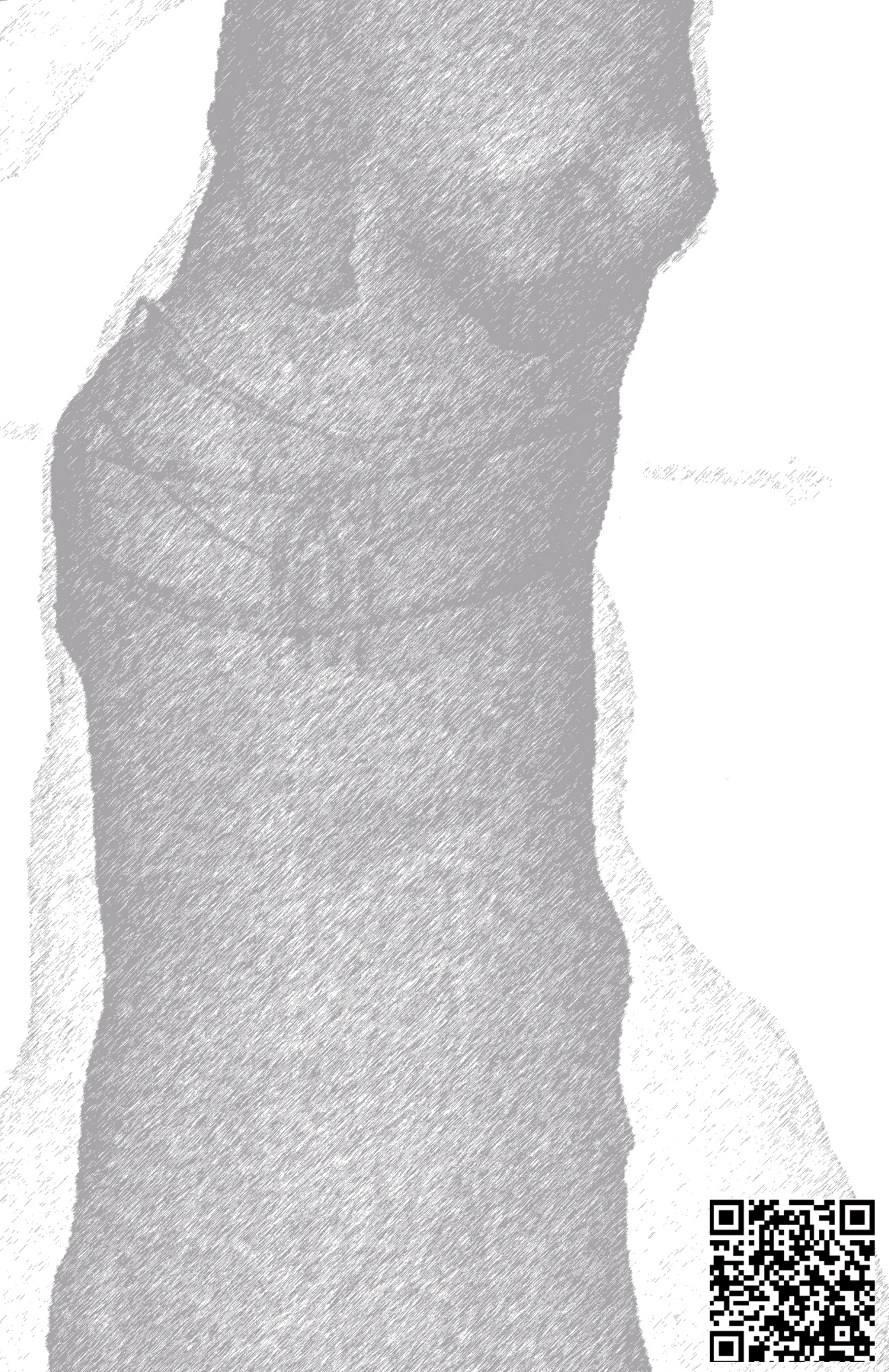

ISBN: $978-90-365-4875-5$ 


\section{Stellingen}

behorende bij het proefschrift

\section{On evaluating stent-artery interaction in abdominal aortic stent grafting}

An in-depth analysis of longitudinal and pulsatility related behavior

\section{Maaike Koenrades}

1. Het verkrijgen van inzicht in de voortgaande interactie tussen endoprothese en bloedvat is essentieel om oorzaken van falen te kunnen begrijpen en falen te voorkomen in toekomstige endoprothesen. - Dit proefschrift

2. Een geïmplanteerde stent kan gedurende 10 jaar een afstand van ongeveer 1200 $\mathrm{km}$ afleggen onder invloed van de pulserende bloedstroom; dit is onmiskenbaar van invloed op het lange termijn functioneren en moet niet worden onderschat. - Dit proefschrift

3. Evaluatie van ECG getriggerde CT scans zou een standaard onderdeel moeten zijn van EVAR management en een vereiste in evaluaties van nieuw geïntroduceerde endoprothesen of aangepaste stentontwerpen; alleen CE certificering met een korte termijn follow-up bij proefpersonen voldoet niet. - Dit proefschrift

4. Een zorgvuldige evaluatie van een kleine groep patiënten kan waardevoller zijn dan evaluatie van een beperkt aantal uitkomstvariabelen op een grote dataset. - Dit proefschrift

5. Niet eerder dan dat de lange termijn prestaties van EVAR gelijk zijn aan die van open operatie, zal EVAR de gouden standaard worden.

6. Wat voor een vaatchirurg als gunstig gezien wordt, kan voor een ingenieur zorgwekkend zijn en vice versa.

7. "Be tenacious. Try to mine all of the information from data, even if it pushes you in the direction of speculation. Many authors are too quick to give up on what they can learn from data". - S. Senturia, JMEMS Editorial, June 2003, Volume 12, Issue 3

8. Nuchter zijn en twijfelen, dat is de kern van wijsheid. - Epicharmus

9. Als IKEA een endoprothese op de markt zou brengen zou dit een "Åderlåding" zijn. 